Pacific Northwest

National Laboratory

Operated by Battelle for the

U.S. Department of Energy

\section{Feed Variability and Bulk Vitrification Glass Performance Assessment}

\author{
L. A. Mahoney \\ J. D. Vienna
}

January 2005

Prepared for the U.S. Department of Energy under Contract DE-AC05-76RL01830 


\title{
DISCLAIMER
}

This report was prepared as an account of work sponsored by an agency of the United States Government. Neither the United States Government nor any agency thereof, nor Battelle Memorial Institute, nor any of their employees, makes any warranty, express or implied, or assumes any legal liability or responsibility for the accuracy, completeness, or usefulness of any information, apparatus, product, or process disclosed, or represents that its use would not infringe privately owned rights. Reference herein to any specific commercial product, process, or service by trade name, trademark, manufacturer, or otherwise does not necessarily constitute or imply its endorsement, recommendation, or favoring by the United States Government or any agency thereof, or Battelle Memorial Institute. The views and opinions of authors expressed herein do not necessarily state or reflect those of the United States Government or any agency thereof.

\author{
PACIFIC NORTHWEST NATIONAL LABORATORY \\ operated by \\ BATTELLE \\ for the \\ UNITED STATES DEPARTMENT OF ENERGY \\ under Contract DE-AC05-76RL01830
}

ك 


\title{
Letter Report
}

\section{Feed Variability and Bulk Vitrification Glass Performance Assessment}

\author{
L. A. Mahoney \\ J. D. Vienna
}

January 2005

Prepared for

the U.S. Department of Energy

under Contract DE-AC05-76RL01830

Pacific Northwest National Laboratory

Richland, Washington 99352 
PNNL-14985 Rev. 0 


\section{Summary}

The Low-Activity Waste (LAW) Supplemental Treatment Demonstration Project is pursuing testing and demonstration of several supplemental treatment technologies. One of these is bulk vitrification (BV), also known as in-container vitrification $\left(\mathrm{ICV}^{\mathrm{TM}}\right)^{(\mathrm{a})}$. Because of the variability of the Hanford tank wastes, the supplemental treatment (ST) ICV process must be designed to handle a range of feed compositions. One purpose of this letter report is to describe the compositional variability of the feed to ST and determine which compositions should be included in an ICV testing program. The other is to support the Tri-Party Agreement M-62-08 milestone decision ${ }^{(\mathrm{b})}$ by making initial estimates of the amount of glass that will be produced with ICV from the ST feed. These initial estimates will help establish whether ICV is a practical treatment methodology for all Hanford tank wastes.

Roughly nine-tenths of the ST LAW feed will come from the Waste Treatment Plant (WTP) pretreatment process. This portion of the ST LAW processed waste varies over time and is expected to consist of 1) a portion of the same LAW feed sent to the WTP melters and 2) a dilute stream that is the product of the condensate from the submerged-bed scrubber (SBS) and the drainage from the wet electrostatic precipitator (WESP), both of which are part of the LAW off-gas system. As in the preceding report, ${ }^{(\mathrm{c})}$ the compositions of all WTP-supplied streams were predicted by the WTP program's dynamic process flowsheet model $\left(\mathrm{G} 2^{\mathrm{TM}}\right)^{(\mathrm{d})}$ for the whole WTP campaign. This portion of the ST LAW feed consisted of 1451 batches of varying composition.

The interface between WTP and ST has not been determined. Therefore there is no set design for the methods of concentrating the off-gas product stream and of combining the excess LAW and off-gas product streams. One possible arrangement, the only one considered in this report, would add half of the total LAW to the off-gas product stream. (Total LAW equals that portion of LAW sent to the WTP LAW vitrification plant [WTP LAW] plus the LAW not currently treatable in the LAW vitrification plant due to capacity limitations [excess]).

The ST feed that does not come from WTP will come from single-shell tanks (SSTs) that have been determined to contain low-curie wastes. These wastes will not undergo separations within the WTP pretreatment facility; however, selective dissolution ${ }^{(\mathrm{e})}$ will be applied to the wastes to reduce the soluble radionuclides (e.g., Cs-137, Tc-99 and I-129) that are processed by ST. The list of tanks designated as containing low-curie wastes has changed over time, a change which has an impact on the analysis of lowcurie wastes in this report. The TFCOUP5A feed vector used to make the G2 run excluded waste from 20 SSTs; at the time the vector was generated, these tanks were considered to contain low-curie wastes that would be sent to ST without separations in the WTP pretreatment facility. These 20 tanks completed the set of predicted ST feeds, consistent with the assumptions that went into the feed vector. In addition, since the time the TFCOUP5A vector was generated, 16 tanks that were not part of the excluded set have

(a) "ICV" is a trademark of AMEC Incorporated, Tempe, Arizona.

(b) HFFACO. 1988. Hanford Federal Facility Agreement and Consent Order as amended, informally known as the Tri-Party Agreement or TPA. This agreement is among the U.S. Department of Energy, the U.S. Environmental Protection Agency, and Washington State Department of Ecology.

(c) Mahoney, L.A. 2004. Waste Simulant Formulation for Series-22 Bulk Vitrification Tests, letter report ST05.004, Pacific Northwest National Laboratory, Richland, Washington. November 12, 2004.

(d) "G2" is a trademark of the GENSYM Corporation, Burlington, Massachusetts. 
been determined to contain low-curie waste. ${ }^{(e)}$ Although the wastes in these 16 tanks were part of the G2 model input and therefore made up part of the WTP-derived ST feed, this report also considers these wastes as potential contributors to the non-WTP-treated ST feed. The Best Basis Inventory (BBI) and the BBI water wash factors for the wastes in the 36 selected tanks, together with non-tank-specific selective dissolution factors defined by retrieval experience and modeling, were used to calculate a set of 36 feed streams to ST in addition to the 1451 batches predicted to come from WTP pretreatment.

All of the streams fed to ST were processed to express the compositions in terms of the concentrations of waste oxides and other constituents. Next, the WTP-derived streams were broken into three broad groups: those dominated by off-gas product composition, those dominated by LAW streams with high fluoride, and those dominated by LAW streams with low fluoride. Then statistical analysis software (JMP ${ }^{\mathrm{TM}(\mathrm{f})}$ ) was used to subdivide the low-fluoride LAW-dominated streams into "clusters" of similar composition. To assess the properties of the glass, similarity was defined according to the three species with the strongest effect on glass durability and phase separation: $\mathrm{Al}_{2} \mathrm{O}_{3}, \mathrm{P}_{2} \mathrm{O}_{5}$, and $\mathrm{SO}_{3}$. The oxides $\mathrm{MoO}_{3}$ and $\mathrm{CaO}$ were omitted as similarity criteria because of the low predicted concentrations. The concentrations of certain other chemicals- $\mathrm{Cl}, \mathrm{NO}_{3}$, and $\mathrm{NO}_{2}$ - were not used as criteria in this cluster analysis but will be considered in defining simulants for engineering-scale melter tests because they relate to overall melter system performance and immobilization success rather than to the glass properties of concern in the crucible tests.

The following conclusions were drawn from the review of feed compositional variability:

- The low-curie tank waste compositions are frequently outside the concentration ranges of most of the LAW streams generated by the WTP pre-treatment facility. Several low-curie wastes are higher in nominal $\mathrm{P}_{2} \mathrm{O}_{5}$ and $\mathrm{SO}_{3}$ than the WTP-derived LAW streams, and most are lower in nominal $\mathrm{Al}_{2} \mathrm{O}_{3}$ and $\mathrm{Cl}$. (The "nominal" concentration of a constituent is that calculated on the basis of $20 \mathrm{wt} \%$ $\mathrm{Na}_{2} \mathrm{O}$.)

- The streams whose composition is dominated by off-gas product contain very little of the $\mathrm{Na}$ and $\mathrm{Al}$ but have high nominal concentrations of most of the significant anion species $\left(\mathrm{Cl}, \mathrm{F}, \mathrm{NO}_{2}, \mathrm{NO}_{3}\right.$, and $\left.\mathrm{SO}_{3}\right)$.

- The maximum nominal concentrations of $\mathrm{CaO}$ and $\mathrm{SO}_{3}$ that, to date, have been tested in glasses are about equal to the Na-weighted averages for LAW streams.

- The maximum tested nominal concentration of $\mathrm{NO}_{2}$ plus $\mathrm{NO}_{3}$ is an upper bound for virtually all of the LAW streams.

- The concentrations of $\mathrm{Al}_{2} \mathrm{O}_{3}, \mathrm{Cl}, \mathrm{F}$, and $\mathrm{P}_{2} \mathrm{O}_{5}$ in most of the predicted LAW streams are well above the concentrations that have been tested in glasses.

The analysis showed that 16 cluster-average compositions would adequately describe the streams containing more than $99 \%$ of the Na that might be directed to ST from the WTP. These streams are

(e) Raymond, R.E. 2004. Candidate Single-Shell Tanks for Low-Curie Feed to Supplemental Treatment, letter report 7F300-04-RER-001 R1, CH2MHILL Hanford Group, Inc., October 20, 2004.

(f) JMP is a trademark of SAS Institute, Inc., Cary, North Carolina. 
dominated by the contribution from pretreated LAW waste. The small percentage remaining is dominated by the off-gas product and would require considerably different handling for successful vitrification. One composition is provided to describe this type of ST feed, for a total of 17 compositions representing WTP-derived feeds. Eight additional cluster compositions were derived to describe the lowcurie SST waste streams.

To estimate the effectiveness of ICV over the range of ST feed compositions, the glass mass produced for each waste cluster was calculated according to "conservative," "best-estimate," and "stretch" assumptions for acceptable glass compositions. The assumptions were based on a review of glass data that have been generated in support of the ST project along with data from other pertinent projects and general glass chemistry knowledge. Two different combinations of soil and additives were considered, one using standard Hanford soil (together with $\mathrm{B}_{2} \mathrm{O}_{3}$ and $\mathrm{ZrO}_{2}$ ) and one with soil plus a $\mathrm{SiO}_{2}$ supplement (together with $\mathrm{B}_{2} \mathrm{O}_{3}$ and $\mathrm{ZrO}_{2}$ ). The following conclusions were drawn from these calculations:

- The present state of knowledge about the effects of glass composition on glass performance and processing can lead to uncertainties of roughly $45 \%$ in glass production, based on a comparison of the results for the "conservative" and "stretch" cases.

- The lowest waste loadings are the result of high sulfur and/or phosphorus concentrations.

- The extremes that are present in the composition of the waste fed to ICV increase the total glass mass by $10 \%$ to $30 \%$, compared to the glass that would be produced if the waste was constantly at the overall-average composition of the feed.

- Adjusting the glass additives with pure $\mathrm{SiO}_{2}$ to optimize waste loading may decrease the total ICVproduced glass mass by $10 \%$ to $15 \%$. 
PNNL-14985 Rev. 0

Page vi of xii 


\section{Contents}

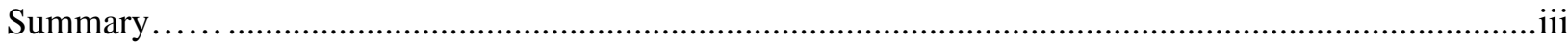

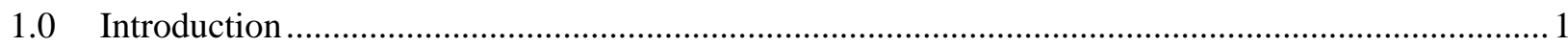

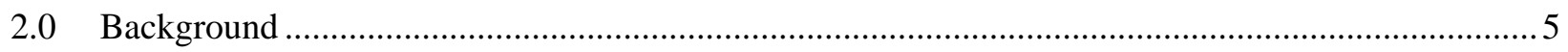

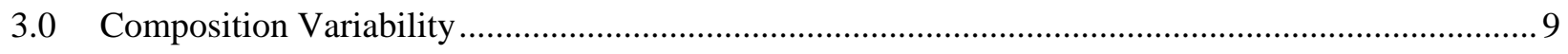

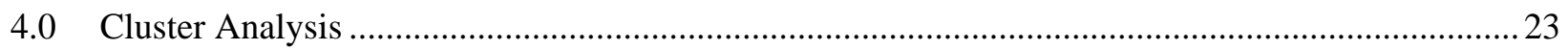

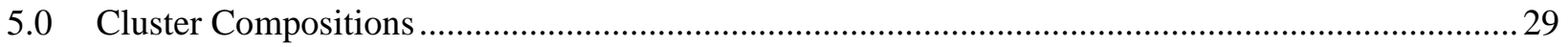

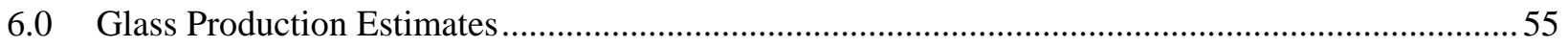

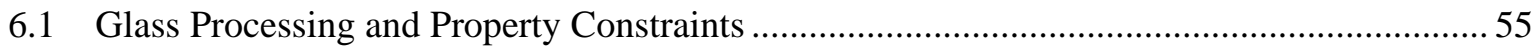

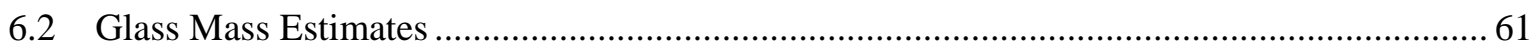

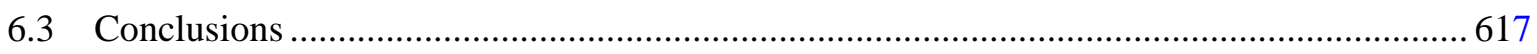

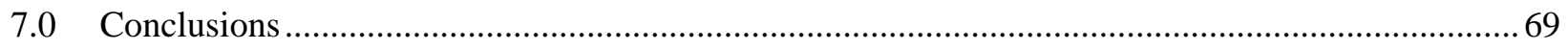

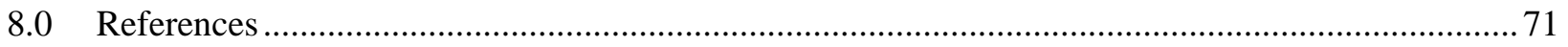

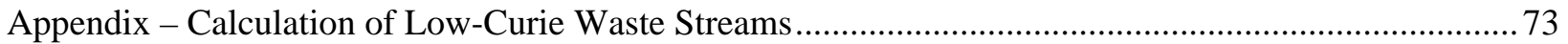




\section{Figures}

1.1. Diagram of Cluster Analysis Procedure ....................................................................................... 2

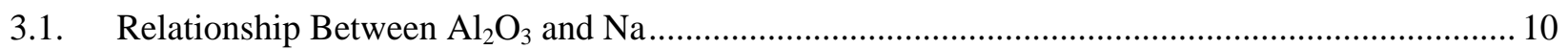

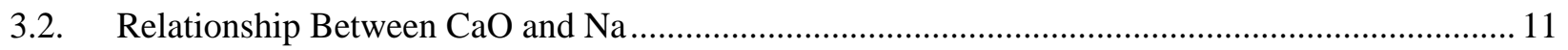

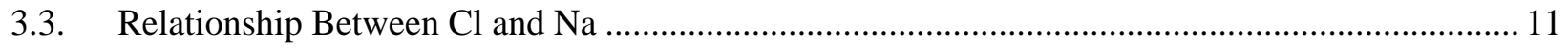

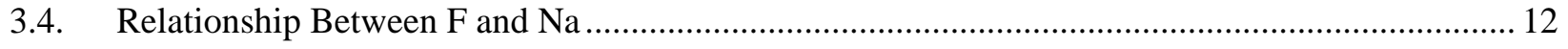

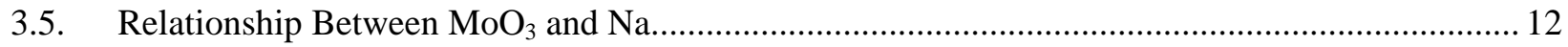

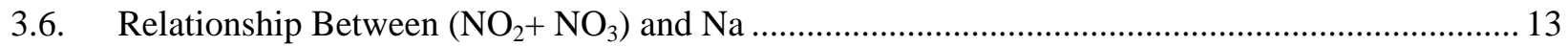

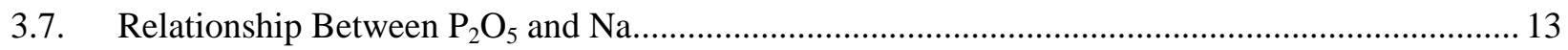

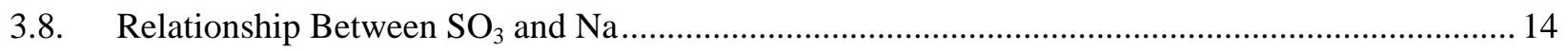

3.9a. Nominal $\mathrm{Al}_{2} \mathrm{O}_{3}$ Concentration Versus Na Mass, by Stream ................................................... 16

3.9b. Close-up of Nominal $\mathrm{Al}_{2} \mathrm{O}_{3}$ Versus Na Mass by Stream ........................................................ 16

3.10a. Nominal Cl Concentration Versus Na Mass by Stream ...............................................................17

3.10b. Close-up of Nominal Cl Versus Na Mass by Stream ............................................................. 17

3.11a. Nominal F Concentration Versus Na Mass, by Stream.............................................................. 18

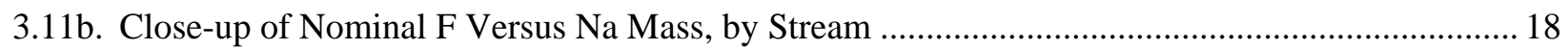

3.12a. Nominal $\left(\mathrm{NO}_{2}+\mathrm{NO}_{3}\right)$ Concentration Versus $\mathrm{Na}$ Mass by Stream ............................................... 19

3.12b. Close-up of Nominal $\left(\mathrm{NO}_{2}+\mathrm{NO}_{3}\right)$ Versus Na Mass by Stream.................................................. 19

3.13a. Nominal $\mathrm{P}_{2} \mathrm{O}_{5}$ Concentration Versus Na Mass by Stream ....................................................... 20

3.13b. Close-up of Nominal $\mathrm{P}_{2} \mathrm{O}_{5}$ Versus Na Mass by Stream ........................................................... 20

3.14a. Nominal $\mathrm{SO}_{3}$ Concentration Versus Na Mass, by Stream ........................................................ 21

3.14b. Close-up of Nominal $\mathrm{SO}_{3}$ Versus Na Mass, by Stream ........................................................... 21

4.1. Distribution of Nominal $\mathrm{Al}_{2} \mathrm{O}_{3}$ Concentrations Among Clusters............................................... 26

4.2. Distribution of Nominal $\mathrm{P}_{2} \mathrm{O}_{5}$ Concentrations Among Clusters ….......................................... 26 
4.3. Distribution of Nominal $\mathrm{SO}_{3}$ Concentrations Among Clusters................................................. 27

6.1. Summary of Tree Analyses of the 39 Glasses from Table 6.2 with $\mathrm{SiO}_{2} \geq 40 \mathrm{wt} \% \ldots \ldots \ldots \ldots \ldots \ldots \ldots . . . . . . . .60$

6.2. Plot of Glass Mass Estimate Ranges for Standard Additives.................................................... 655

6.3. Plot of Glass Mass Estimate Ranges for Alternative Additives ............................................... 677 


\section{Tables}

3.1. Summary of Significant Constituent Distributions ….............................................................. 14

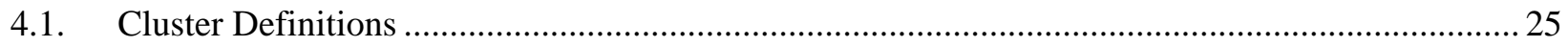

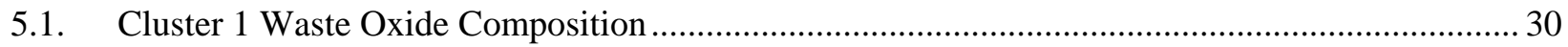

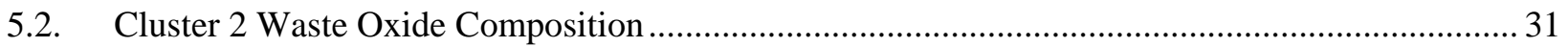

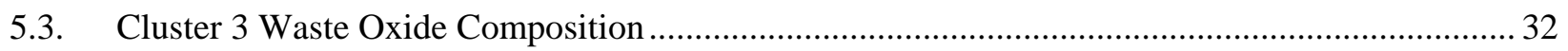

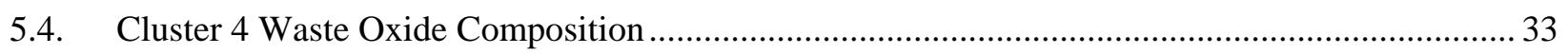

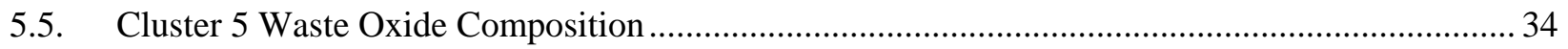

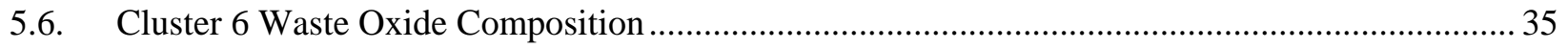

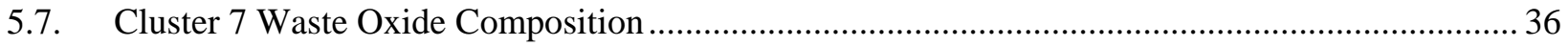

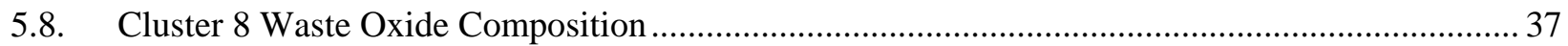

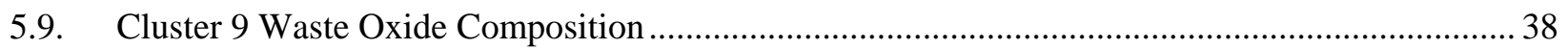

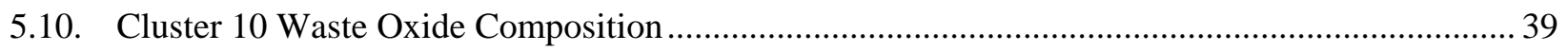

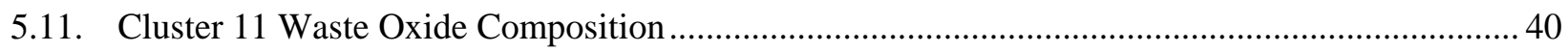

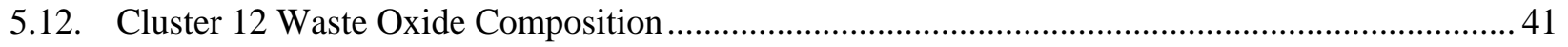

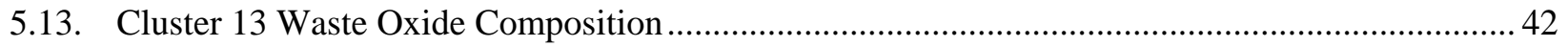

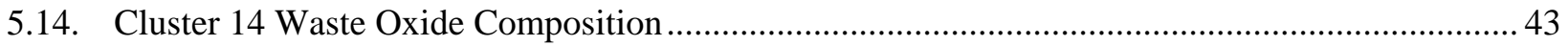

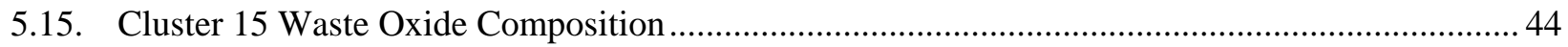

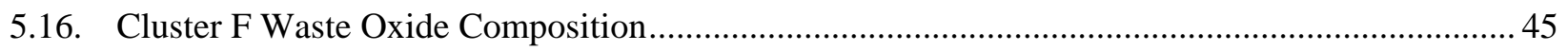

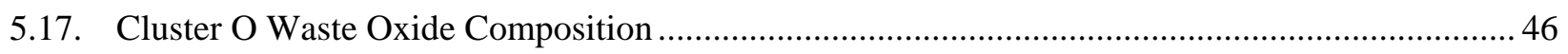

5.18. Cluster Y1 Waste Oxide Composition ................................................................................... 47

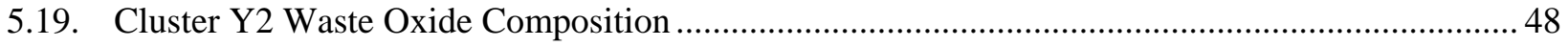

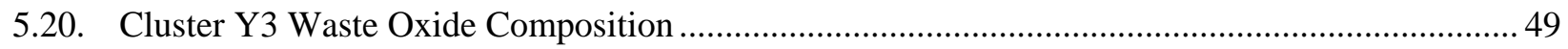

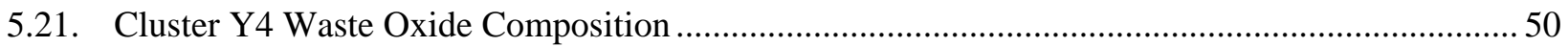




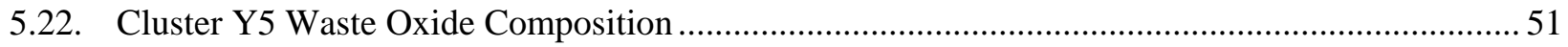

5.23. Cluster Y6 Waste Oxide Composition ...................................................................................... 52

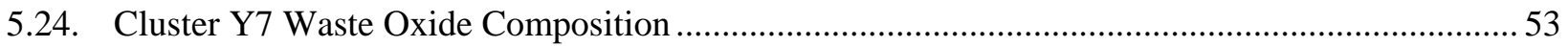

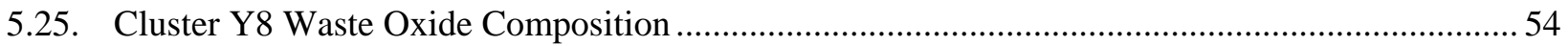

6.1. Summary of Key Component Effects on Soda-Aluminosilicate Glass Properties..........................57

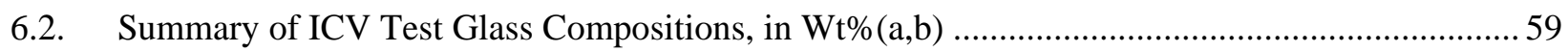

6.3. Summary of Glass Component Constraints (values in wt\% on a glass basis) ............................... 62

6.4. Soil Composition Used in Glass Calculations (Kim et al. 2003) ...............................................62

6.5. Summary of Estimated Waste Loadings and Glass Masses for Standard Additives(a,b) ............... 64

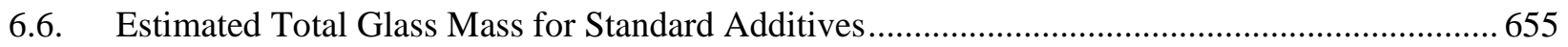

6.7. Summary of Estimated Waste Loadings and Glass Masses for Alternative Additives(a,b) ......... 666

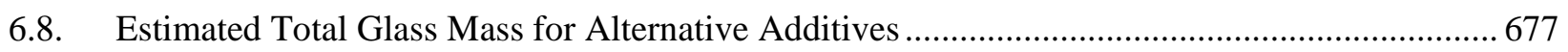


PNNL-14985 Rev. 0

Page xii of xii 


\subsection{Introduction}

The Low-Activity Waste (LAW) Supplemental Treatment Demonstration Project is pursuing testing and demonstration of several supplemental treatment technologies. Bulk vitrification (BV), also known as in-container vitrification (ICV), was one of the technologies evaluated for their potential to supplement the treatment capacity of the Hanford Waste Treatment and Immobilization Plant (WTP) for LAW. In 2003, CH2M HILL Hanford Group, Inc, made an investment decision to pursue a pilot-scale test and demonstration facility for bulk vitrification treatment of selected Hanford low-activity tank waste. The test and demonstration facility is planned to be operational late in 2005, and will produce up to 50 boxes of vitrified material to provide data to support final decisions on tank waste treatment. The tank waste for the test and demonstration facility will come from Hanford Tank 241-S-109 (S-109). This waste will be used directly in the initial boxes to define acceptable operational windows. Later testing will produce boxes with S-109 waste spiked with other chemicals to represent other tank wastes expected to be treated with ICV.

A number of smaller-scale simulant tests are planned to support the test and demonstration facility. The S-109 simulant used in these tests was described in earlier letter reports. ${ }^{\text {(a) }}$ Other Supplemental Treatment (ST) tests require simulants that represent the range of compositions that might be present in waste fed to ST throughout the Waste Treatment Plant (WTP) campaign. The Series 33 tests to be performed in the engineering-scale melter (ESM) are limited to three to five simulants, one of which is the already-defined S-109 waste simulant. Before the Series 33 tests take place, the Series 22 tests will be performed at crucible scale to determine glass properties for a range of glasses that describe the ST feed. A preceding report ${ }^{(b)}$ set out the information needed for the Series 22 tests, a set of 21 waste formulations expressed in terms of waste-oxide composition.

One purpose of this letter report is to describe the compositional variability of the feed to ST and show which compositions are most relevant to ICV testing. Much of the variability and feed composition information in this report was also presented in the preceding report; however, this study considers a larger set of feeds from low-curie single-shell tank (SST) wastes, and includes the effect of selective dissolution during retrieval. The other purpose of this report is to provide an initial assessment of the performance of ICV in handling the entire ST feed envelope. Such an assessment is needed to support the Tri-Party Agreement M-62-08 milestone decision by determining which wastes are likely to create difficulties for ICV. To this end, the amount of glass required to immobilize the ST feed is calculated for groupings of feed streams that represent the entire envelope of compositions.

Figure 1.1 diagrams the procedure used in defining the waste-oxides formulations that are one of the products of the current study. The ST feeds that originate in the WTP pretreatment facility are 1451 weekly streams calculated with the G2 model of the WTP facility. Half of the predicted total LAW is sent to the WTP melters and half is sent to ST, where it is combined with a stream produced by the WTP LAW off-gas process. The reason for using this particular combination is given in Section 2 of this report.

(a) Mahoney, L.A. and S.D. Rassat. December 5, 2003. Tank 241-S-109 Cold Saltcake Simulant Formulation, letter report ST04.007, Pacific Northwest National Laboratory, Richland, Washington. Bagaasen, L.M. January 21, 2004. Baseline S-109 Chemical Simulant Recipe for AMEC Tests, letter report ST04.010, Pacific Northwest National Laboratory, Richland, Washington.

(b) Mahoney, L.A. November 12, 2004. Waste Simulant Formulation for Series-22 Bulk Vitrification Tests, letter report ST05.004, Pacific Northwest National Laboratory, Richland, Washington. 
G2 MODEL OUTPUT -- 1451 WEEKS

(not all LAW tanks are in WTP feed)

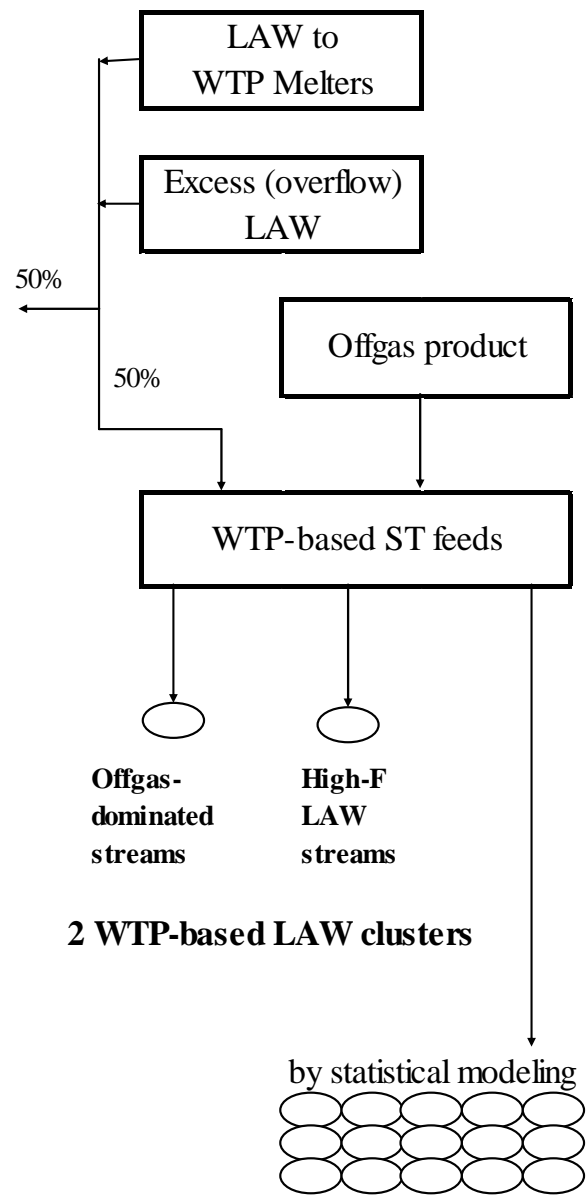

\section{5 more WTP-based \\ LAW clusters}

BEST BASIS INVENTORIES (BBIs)

(36 low-Ci SSTs)

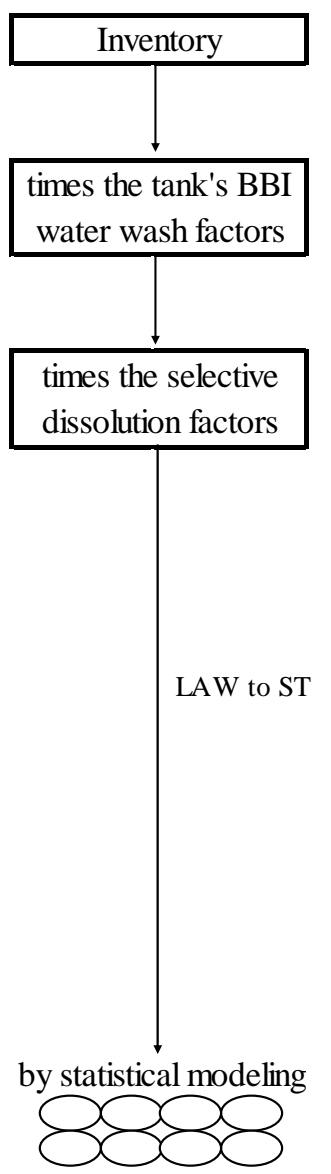

8 tank-based

LAW clusters

Figure 1.1. Diagram of Cluster Analysis Procedure

As described in Section 3, the WTP-derived feeds are subdivided into three parts - streams dominated by off-gas product, streams dominated by LAW waste with high fluoride, and streams dominated by LAW waste with low fluoride. This last part, which makes up most of the WTP-derived feed, is subdivided by statistical analysis into 15 clusters, as described in Section 4. These WTP-derived clusters contain the same streams and have the same compositions as in the preceding Series-22 report. ${ }^{\text {(a) }}$

Figure 1.1 also shows the procedure used for the 36 SST wastes that were assumed to be sent to ST after undergoing pretreatment outside of the WTP plant. The LAW streams from the 36 SSTs were calculated as being the water-soluble portion of the Best Basis Inventories (BBIs) that was retrieved from the tanks during the later part of retrieval, after selective removal of the relatively high-activity liquid had

(a) Mahoney, L.A. November 12, 2004. Waste Simulant Formulation for Series-22 Bulk Vitrification Tests, letter report ST05.004, Pacific Northwest National Laboratory, Richland, Washington. 
taken place. The SST LAW wastes did not fit within WTP-based clusters; they were statistically subdivided into 8 clusters. These 8 clusters (identified as Y1 through Y8) replace the 4 low-curie waste clusters that were defined in the preceding Series-22 report (identified as X1 through X4).

The average compositions of the 25 clusters defined in this report were calculated in terms of waste oxide and are presented in Section 5. Then the mass of glass required for each cluster was determined for "conservative," "best estimate," and "stretch" formulations for each of two different mixtures of Hanford soil and additives. The results of these calculations are given in Section 6. 
PNNL-14985 Rev. 0

Page 4 of 78 


\subsection{Background}

The currently defined waste streams that are potentially directed to ST include the following:

- Some portion of the LAW stream produced by the WTP pretreatment process. LAW is strongly alkaline with a total Na concentration in the range of 5 to $10 \mathrm{M}$. It is not yet determined whether the LAW sent to ST will be 1) a fraction of what is sent to the WTP melters, thus having the same range of compositions as the WTP melter feed; 2) only the excess LAW over what can be stored, which will not be available to ST at all times and so will only include a subset of the WTP LAW compositions; or 3) the LAW streams whose compositions are the most challenging to the WTP melters (for example, high- $\mathrm{SO}_{4}$ streams) but are not representative of LAW as a whole.

- The submerged-bed scrubber (SBS) condensate streams, which originate in the WTP LAW process off-gas system and are dilute alkaline solutions, relatively high in $\mathrm{Cl}, \mathrm{F}, \mathrm{NO}_{3}, \mathrm{NO}_{2}$, and $\mathrm{SO}_{4}$.

- The wet electrostatic precipitator (WESP) streams, dilute and near-neutral pH solutions, which also come from the WTP LAW process off-gas system.

- Demineralized water used to adjust WTP LAW off-gas condensate chemistry.

- Low-activity waste from low-curie saltcake tanks that is sent to ST without being routed through WTP pretreatment. This type of waste will be similar in nature to the LAW, but with lower concentrations of $\mathrm{Al}$ and higher concentrations of $\mathrm{SO}_{4}$ and $\mathrm{PO}_{4}$.

Other as-yet undefined streams may also be included; for example, the liquids produced by the ST ICV off-gas system may be recycled into the ST feed. At this time, there is no information about the composition or volume of any such additional streams.

The interface between WTP and ST also remains to be defined. This report, like the preceding Series-22 report, considers an interface design in which 50 percent of the concentrated LAW is sent to ST during all periods when LAW is being produced. There the LAW is mixed with off-gas product (SBS condensate, WESP solution, and demineralized water) that has been separately concentrated by ST. Because LAW is not produced at all times, there are some periods when the ST feed is pure off-gas product.

The best available source of predicted WTP stream compositions is a recent run of the WTP G2 dynamic flowsheet model $^{(a)}$ (as described in 24590-WTP-MRR-PO-04-0011, Rev. 0). The run provided output at weekly intervals for the mass of each constituent that made up the WTP LAW, excess LAW, ${ }^{\text {(b) }}$ and off-gas product streams. This resulted in 1451 discrete batches for each stream. ${ }^{\text {(c) }}$ The first week of

(a) Model run request 24590-WTP-MRQ-PO-04-0665, Supplemental LAW Data Collection. Run results were transmitted to the U.S. Department of Energy, Office of River Protection on September 16, 2004. The software run was the Dynamic (G2) Model, version 3.1 (with run-specific changes described in the run request).

(b) "Excess LAW" refers to the overflow from the storage tanks that supply the WTP melters. Adding excess LAW to WTP LAW gives the total LAW produced by pretreatment.

(c) The G2 model identifiers for the streams were V41001_V21001, V41001_V21002 (the two WTP LAW melter feeds), V41001_LAW-OVERFLOW-1 (the excess LAW to ST), and V25003_LAW-OVERFLOW-2 (the offgas product coming from vessel RLD-VSL-00005). 
output was December 7, 2009, and the last was September 28, 2037. The end-of-mission date was later than 2028, which was accepted because the run was intended only to predict the overall population of feed compositions, not to indicate the anticipated time period to treat all the tank waste.

The G2 run used the TFCOUP5A feed vector (Kirkbride et al. 2003) and its associated leach factors, together with run parameters that represent the latest dates of hot commissioning, hot operation, and ramp up, and the associated melter production rates (within the design limitations of the WTP). Some of the characteristics of the run that should be kept in mind in interpreting the predicted compositions are

- The TFCOUP5A feed vector excludes from LAW pretreatment the soluble wastes from the 20 tanks that at the time of the vector's generation were considered to contain low-curie wastes that could be sent directly to ST without pretreatment in the WTP plant. The excluded tanks were B-101, B-102, B-103, B-105, B-106, B-107, B-108, B-109, BX-103, BX-107, BX-108, BX-109, BX-110, BY-102, BY-105, BY-108, BY-111, BY-112, S-109, and TY-102. ${ }^{\text {(a) }}$ Only the insoluble fraction of these tanks (i.e. sludge) is sent to the WTP for processing.

- Constituent concentrations that are derived from supplemental BBI information (including Ag, As, $\mathrm{Ba}, \mathrm{Cd}, \mathrm{Mo}, \mathrm{Sb}, \mathrm{Se}$, and Tl) should be interpreted with great caution. The Se concentrations, in particular, were represented in the TFCOUP5A feed vector by minimum detection limits for the ICP analytical results. This resulted in very high upper bounds for the Se concentrations in the wastes for which ICP fusion digestions were used. ${ }^{(b)}$

- A new LAW glass model for waste loading was used to hold the $\mathrm{Na}_{2} \mathrm{O}$ concentration in glass to 20 wt\%, except when it had to be forced lower to keep the $\mathrm{SO}_{3}$ concentration in LAW glass to a maximum of $0.8 \mathrm{wt} \%$.

- The time necessary to process the feed was calculated with G2 as 28 years, with calculated average ILAW and IHLW production rates of 18.6 and 4.2 metric tons/day, respectively. Envelope C processing was not included in the run, which caused more Sr and TRU to be sent to LAW glass than would have been sent otherwise. In actual processing, the Sr and TRU will be removed from the Envelope C wastes.

- The SBS reactions were updated to adjust melter ammonia production such that the $\mathrm{pH}$ in the SBS would remain in the range of 6.5 to 7.5 .

- The G2 model does not maintain charge balance. The results must be post-processed to balance cations and anions, if a full simulant recipe is required (rather than a waste-oxides mixture formulation).

To complete the TFCOUP5A feed vector, only the wastes from the 20 SSTs indicated above were needed. However, this set of 20 SSTs is not completely consistent with the current plans for low-curie

(a) Personal communication, R. Kirkbride to L.A. Mahoney, regarding TFCOUP5A vector and excluded SSTs, telephone conversation on October 21, 2004.

(b) Personal communication, D.E. Place to L.A. Mahoney, regarding Se inventories as used in TFCOUP5A, e-mail on October 12, 2004. 
feeds to ST. Current plans take into account improved tank waste characterization data, operations in the tank farms, and improved data on selective dissolution during retrieval. ${ }^{\text {(a), (b) }}$

The current set of low-curie waste tanks contains 27 SSTs: B-101, B-103, B-105, B-108, BX-111, BY-102, BY-103, BY-105, BY-108, BY-109, BY-111, BY-112, S-105, S-109, T-109, TX-103, TX-105, TX-108, TX-110, TX-111, TX-112, TX-114, TX-115, TX-117, TX-118, TY-102, and U-107. Eleven of these tanks are among those excluded from the TFCOUP5A feed vector. The other 16 current tanks contain waste that is already part of the WTP-derived waste streams because it was included in the feed vector. However, these 16 wastes also need to be considered under the assumption that they will not pass through WTP pretreatment (although they will be pretreated by solid-liquid separation and selective dissolution). Accordingly, in this report the wastes in these 16 tanks were considered as low-curie feeds, in addition to the 20 excluded SSTs whose wastes were used in the preceding Series-22 report. In some cases, as needed, this report refers to the 16 tanks as "double-counted" tanks and discounts their waste contribution to avoid double-counting.

The insoluble fraction of the waste in the 36 low-curie tank wastes is considered to be sent to the WTP for processing. Selective dissolution is assumed to be applied to the soluble fraction to reduce the soluble radionuclides transferred to the ST.

(a) Raymond, R.E. October 20, 2004. Candidate Single-Shell Tanks for Low-Curie Feed to Supplemental Treatment, letter report 7F300-04-RER-001 R1, CH2MHILL Hanford Group, Inc.

(b) Retrieval that depends on water dissolution of the waste is a partially selective process because the waste interstitial liquid originally present in the waste, with its burden of completely dissolved high-activity constituents such as ${ }^{137} \mathrm{Cs}$ and ${ }^{99} \mathrm{Tc}$, is flushed out of the waste before most of the relatively low-activity solids are dissolved. Hence, the waste retrieved after the initial flushing phase has lower activity and a higher proportion of freshly dissolved salt constituents than does the waste from the initial phase of dissolution retrieval. 
PNNL-14985 Rev. 0

Page 8 of 78 


\subsection{Composition Variability}

The first step in developing simulants was to determine the variability of ST feed composition during the WTP campaign with respect to the waste constituents that most affect glass properties and melter system performance. These significant constituents, and the reasons for their significance, were the following:

- $\mathrm{Al}$, in its oxidized form $\mathrm{Al}_{2} \mathrm{O}_{3}$, affects the durability of the glass

- $\mathrm{Ca}$, in its oxidized form $\mathrm{CaO}$, affects the durability

- $\mathrm{Cl}$ is primarily a melter off-gas concern, but may influence volatility and/or salt phase separation

- F can cause phase separation in glasses and is also a melter off-gas concern

- Mo, in its oxidized form $\mathrm{MoO}_{3}$, can cause phase separation

- $\mathrm{NO}_{2}$ and $\mathrm{NO}_{3}$ affect the volatilization of Tc, the redox balance of the glass, and the off-gas system

- $\mathrm{PO}_{4}$, in its oxidized form $\mathrm{P}_{2} \mathrm{O}_{5}$, can cause phase separation

- $\mathrm{SO}_{4}$, in its oxidized form $\mathrm{SO}_{3}$, can cause phase separation and may influence Tc volatility.

The approach taken to create a population of WTP-originated ST feeds was to assume a plant interface between WTP and ST that sends the off-gas product stream to ST for evaporation and mixes the off-gas concentrate with half the LAW to produce ST feed. The additional LAW feeds from the 36 lowcurie tank wastes were calculated by multiplying the BBI of each constituent by its BBI water wash factor to reflect retrieval by water dissolution, and by a selective dissolution factor to account for the selective removal of initially dissolved constituents of the waste. The Appendix provides more detail about the method of calculating low-curie feed streams.

The total Na sent to the ST, including the 20 excluded low-curie tank wastes but excluding the 16 double-counted tank wastes, was 27998 megagrams (Mg, or metric tons) of Na. Of this, $3168 \mathrm{Mg} \mathrm{Na}$ came from the excluded low-curie tank wastes alone, and $215 \mathrm{Mg}$ Na came from the off-gas product alone. The double-counted tank wastes contained $4725 \mathrm{Mg} \mathrm{Na}$.

For each ST feed stream, the waste oxide composition was calculated in terms of wt $\% \mathrm{Al}_{2} \mathrm{O}_{3}, \mathrm{CaO}$, $\mathrm{Cl}, \mathrm{F}, \mathrm{MoO}_{3}, \mathrm{NO}_{2}+\mathrm{NO}_{3}, \mathrm{P}_{2} \mathrm{O}_{5}$, and $\mathrm{SO}_{3}$ in a waste glass containing a nominal $\mathrm{Na}_{2} \mathrm{O}$ oxide waste loading of $20 \mathrm{wt} \%$. The $\mathrm{wt} \% \mathrm{Na}_{2} \mathrm{O}$ was treated as constant in order to put all the streams on the same wasteloading basis. For the purpose of displaying composition variability, the cation and anion concentrations were used as supplied by the G2 model without imposing a charge-balance requirement. 
Figures 3.1 through 3.8 show how much of the $\mathrm{Na}$ in all streams is associated with different nominal concentrations of each of the significant constituents: $\mathrm{Al}_{2} \mathrm{O}_{3}, \mathrm{CaO}, \mathrm{Cl}, \mathrm{F}, \mathrm{MoO}_{3},{ }^{(\mathrm{a})} \mathrm{NO}_{2}+\mathrm{NO}_{3}, \mathrm{P}_{2} \mathrm{O}_{5}$, and $\mathrm{SO}_{3}$. Note that to show the entire potential composition envelope, the 16 double-counted low-curie SSTs are included in these figures. The total Na represented in each figure is therefore $32723 \mathrm{Mg} \mathrm{Na}$, rather than the $27998 \mathrm{Mg}$ Na present in the ST feed alone.

In Figures 3.1 through 3.8, the columns (which are referred to the left y-axis) show the percentage of $\mathrm{Na}$ in each increment of nominal concentration of the constituent. The curves (which are referred to the right $\mathrm{y}$-axis) represent the percentage of the total $\mathrm{Na}$ in the streams that is present below any given nominal concentration of the significant constituent. In cases where one tank or category of waste is the sole source of a concentration extreme, a note on the figure identifies the source(s) of waste.

For example, Figure 3.1 shows that, if the $\mathrm{Na}$ in all the streams is processed into a $20 \mathrm{wt} \% \mathrm{Na}_{2} \mathrm{O}$ glass waste form, about $25 \%$ of the $\mathrm{Na}$ can be found at nominal $\mathrm{Al}_{2} \mathrm{O}_{3}$ concentrations between $3.0 \mathrm{wt} \%$ and $4.0 \mathrm{wt} \%$ (the sum of the two tallest columns). The curve in the figure indicates that a $20 \mathrm{wt} \% \mathrm{Na}_{2} \mathrm{O}$ glass can hold a total of $85 \%$ of the $\mathrm{Na}$ while having a concentration of less than $5 \% \mathrm{Al}_{2} \mathrm{O}_{3}$ from the waste.

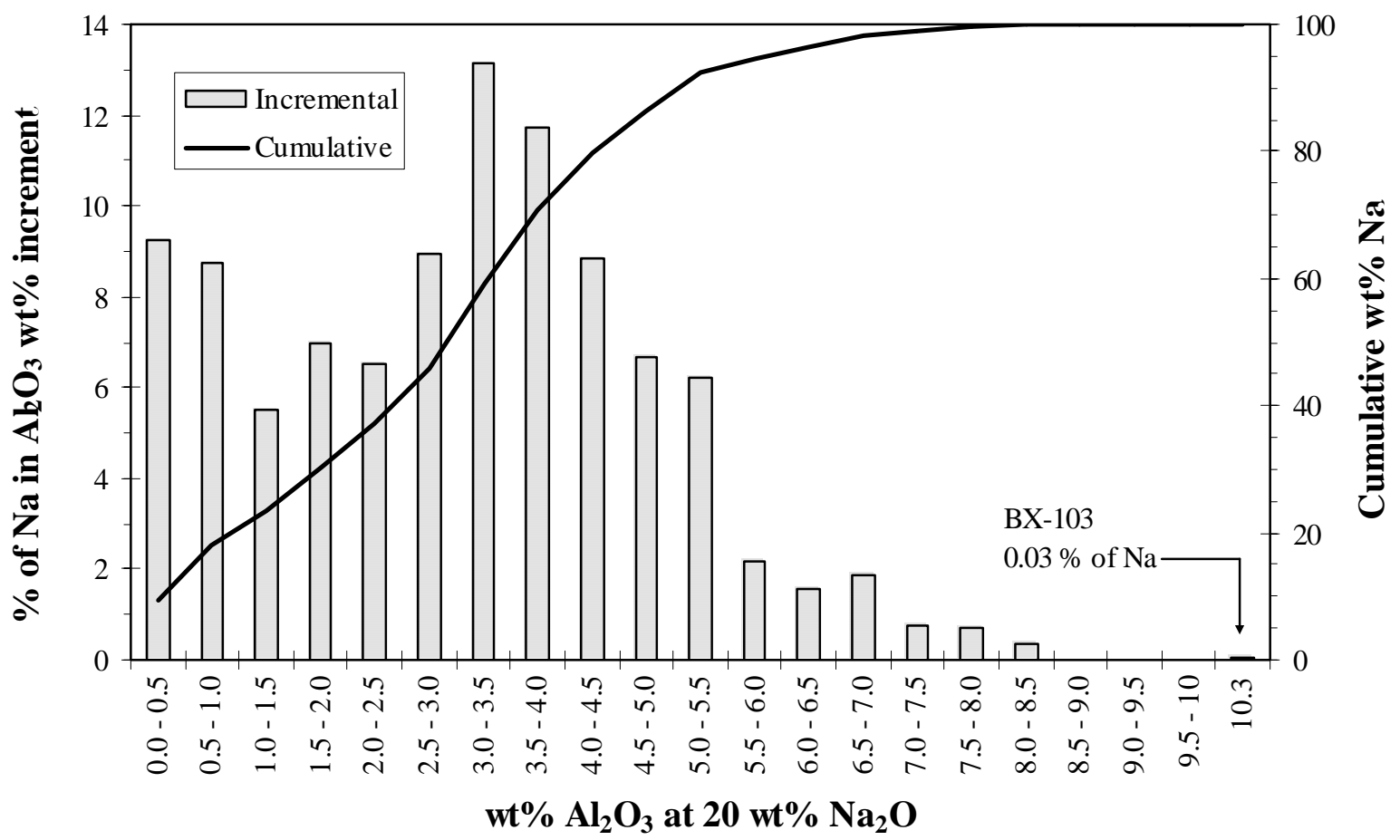

Figure 3.1. Relationship Between $\mathrm{Al}_{2} \mathrm{O}_{3}$ and $\mathrm{Na}$

(a) Only 6 of the BBIs for the 20 excluded low-curie waste tanks included a Mo inventory: tanks B-106, B-107, BX-109, BY-105, BY-108, and S-109. The other 14 excluded SSTs, which contained $6.6 \%$ of the total Na in the ST feed, were assumed to contain no Mo for lack of data in their BBIs. Among the 16 double-counted lowcurie waste tanks, only two (BY-109 and U-107) had Mo data in their BBIs; the others were assumed to contain no Mo. 
PNNL-14985 Rev. 0

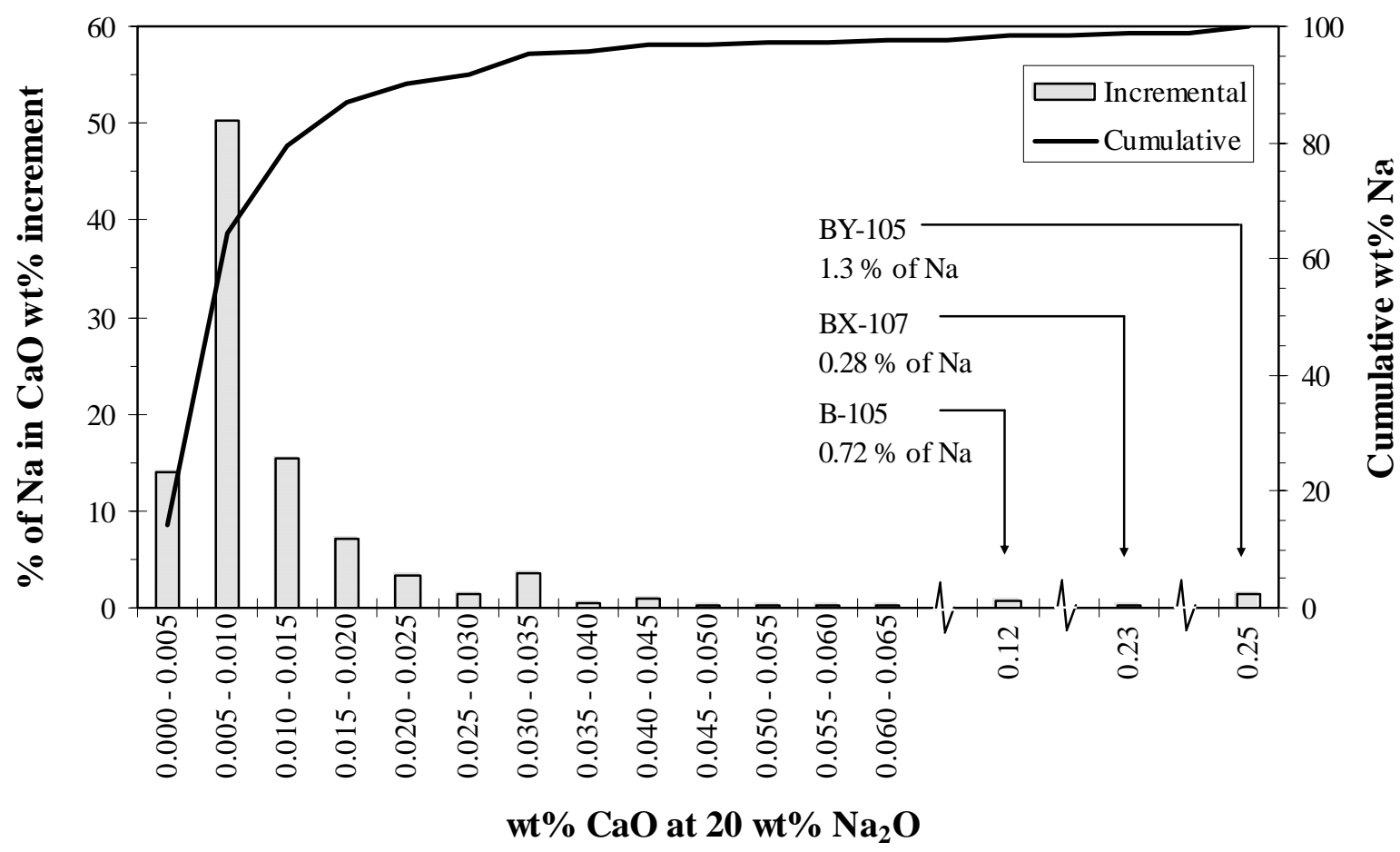

Figure 3.2. Relationship Between $\mathrm{CaO}$ and $\mathrm{Na}$

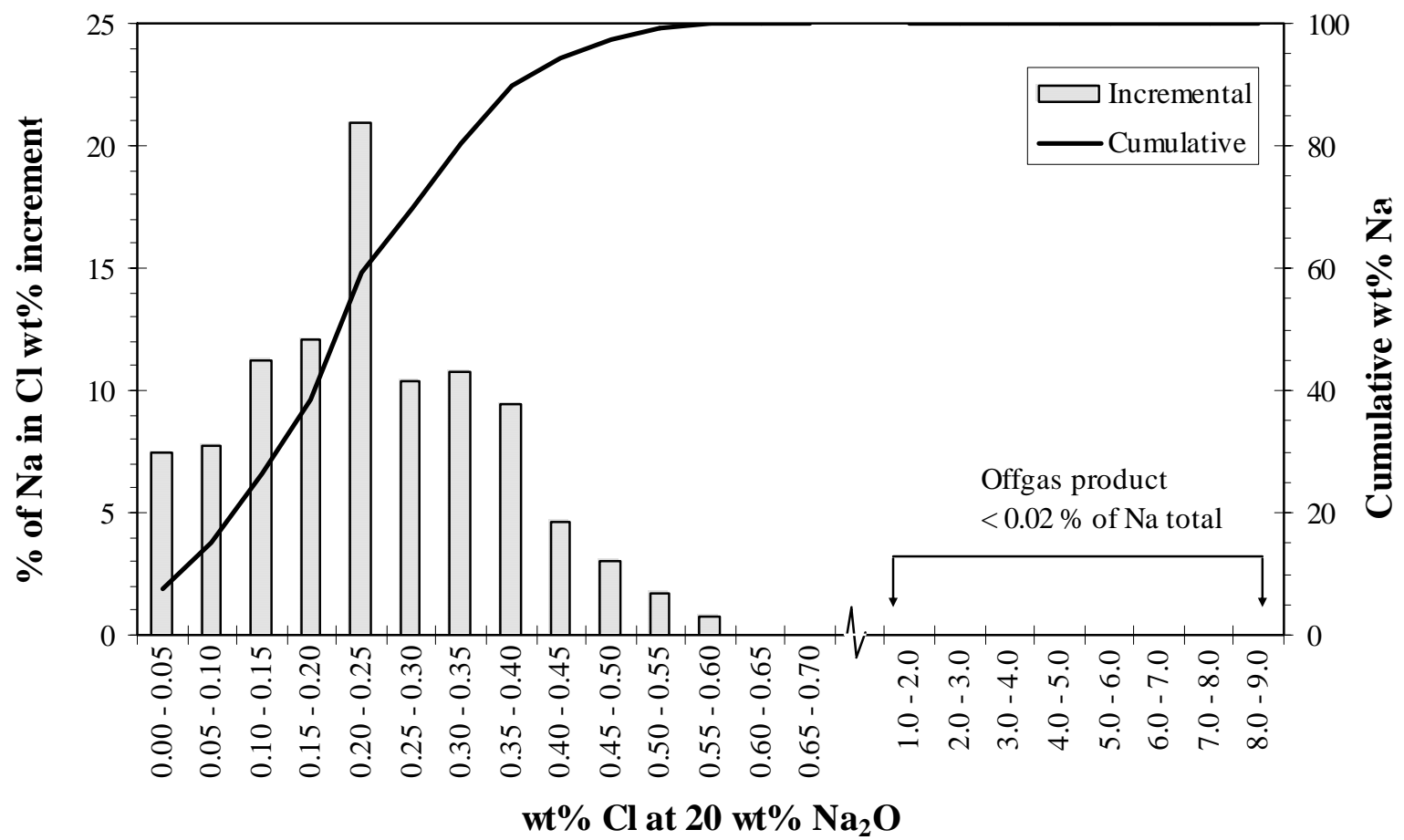

Figure 3.3. Relationship Between $\mathrm{Cl}$ and $\mathrm{Na}$ 
PNNL-14985 Rev. 0

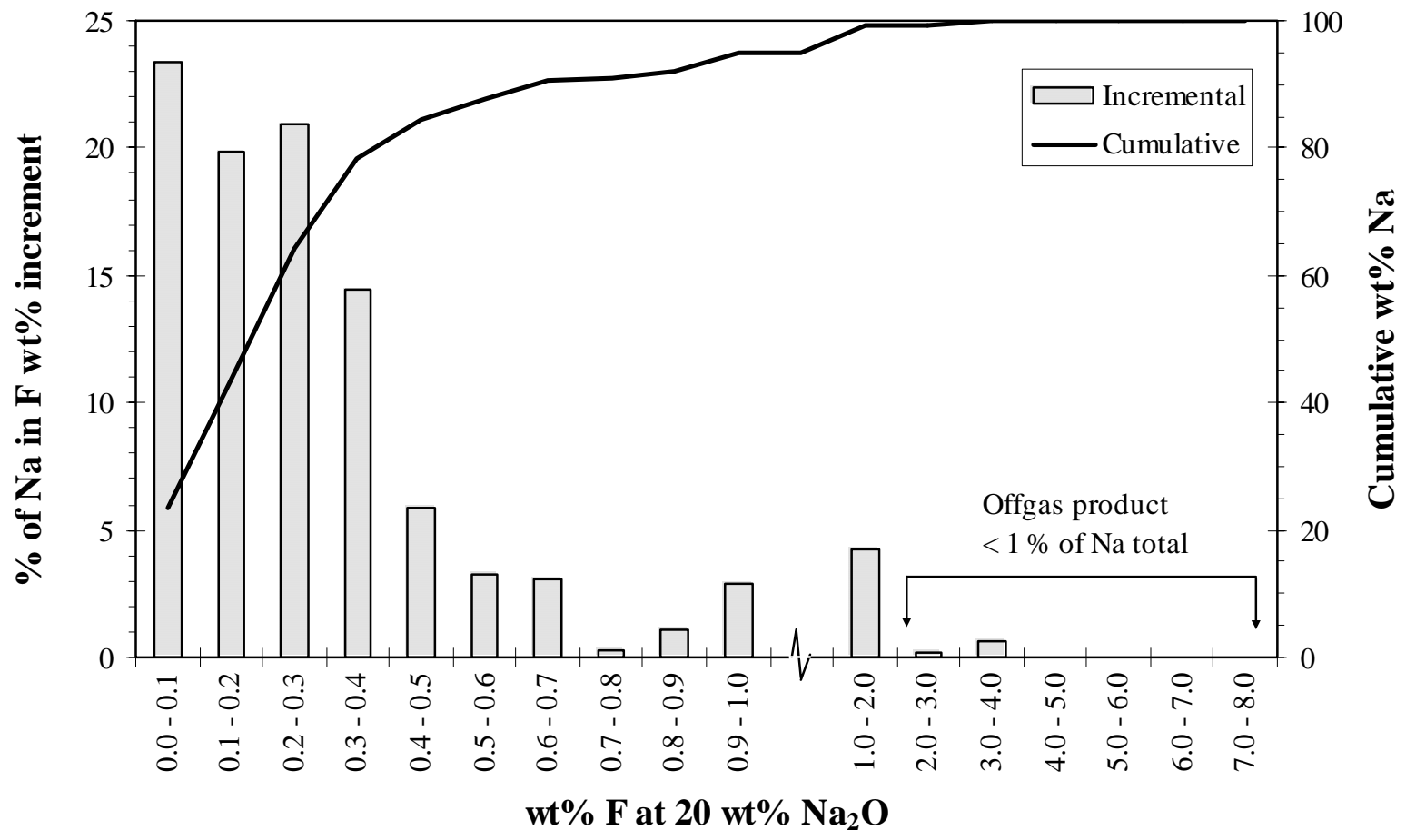

Figure 3.4. Relationship Between $\mathrm{F}$ and $\mathrm{Na}$

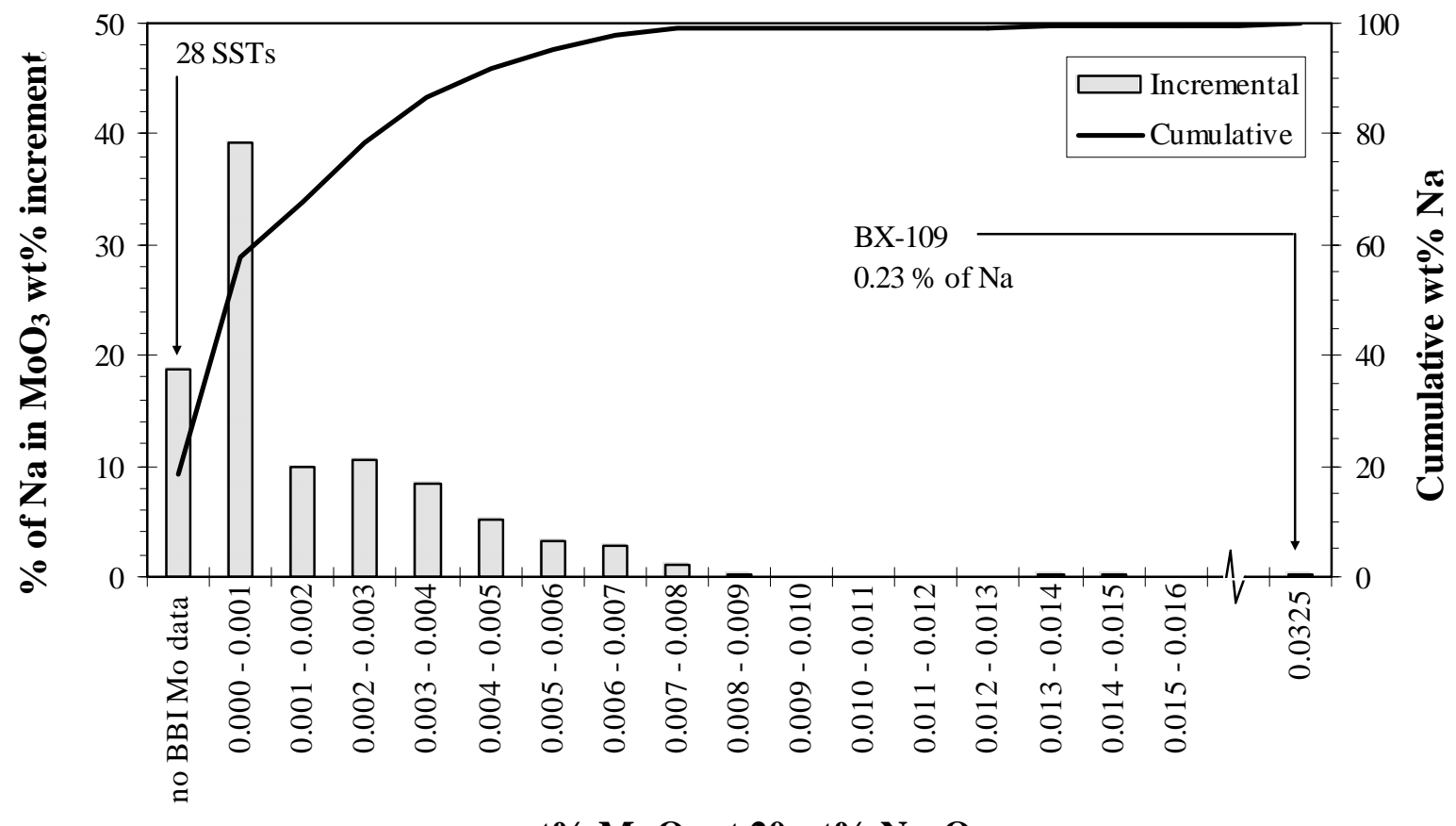

wt $\% \mathrm{MoO}_{3}$ at 20 wt $\% \mathrm{Na}_{2} \mathrm{O}$

Figure 3.5. Relationship Between $\mathrm{MoO}_{3}$ and $\mathrm{Na}$ 
PNNL-14985 Rev. 0

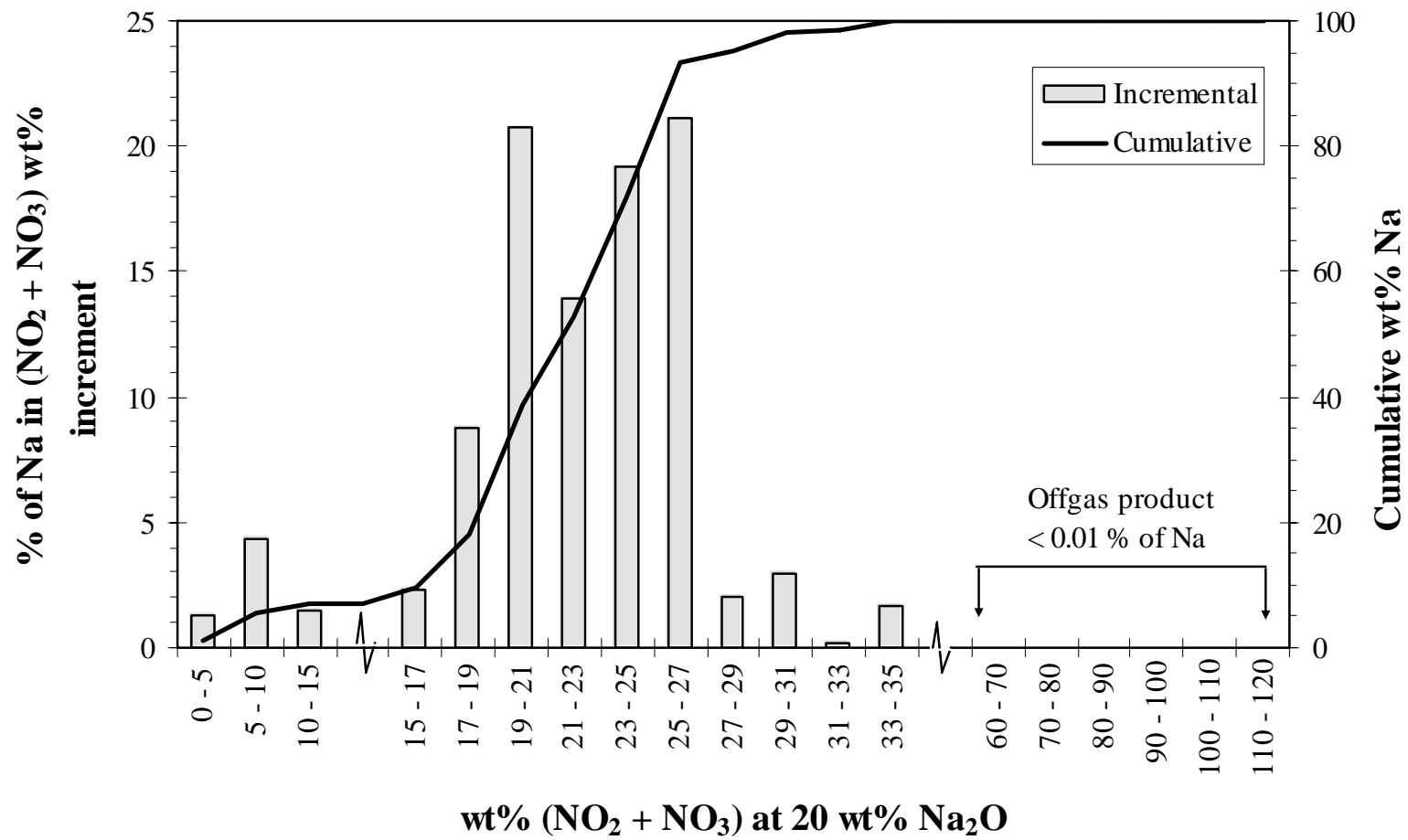

Figure 3.6. Relationship Between $\left(\mathrm{NO}_{2}+\mathrm{NO}_{3}\right)$ and $\mathrm{Na}$

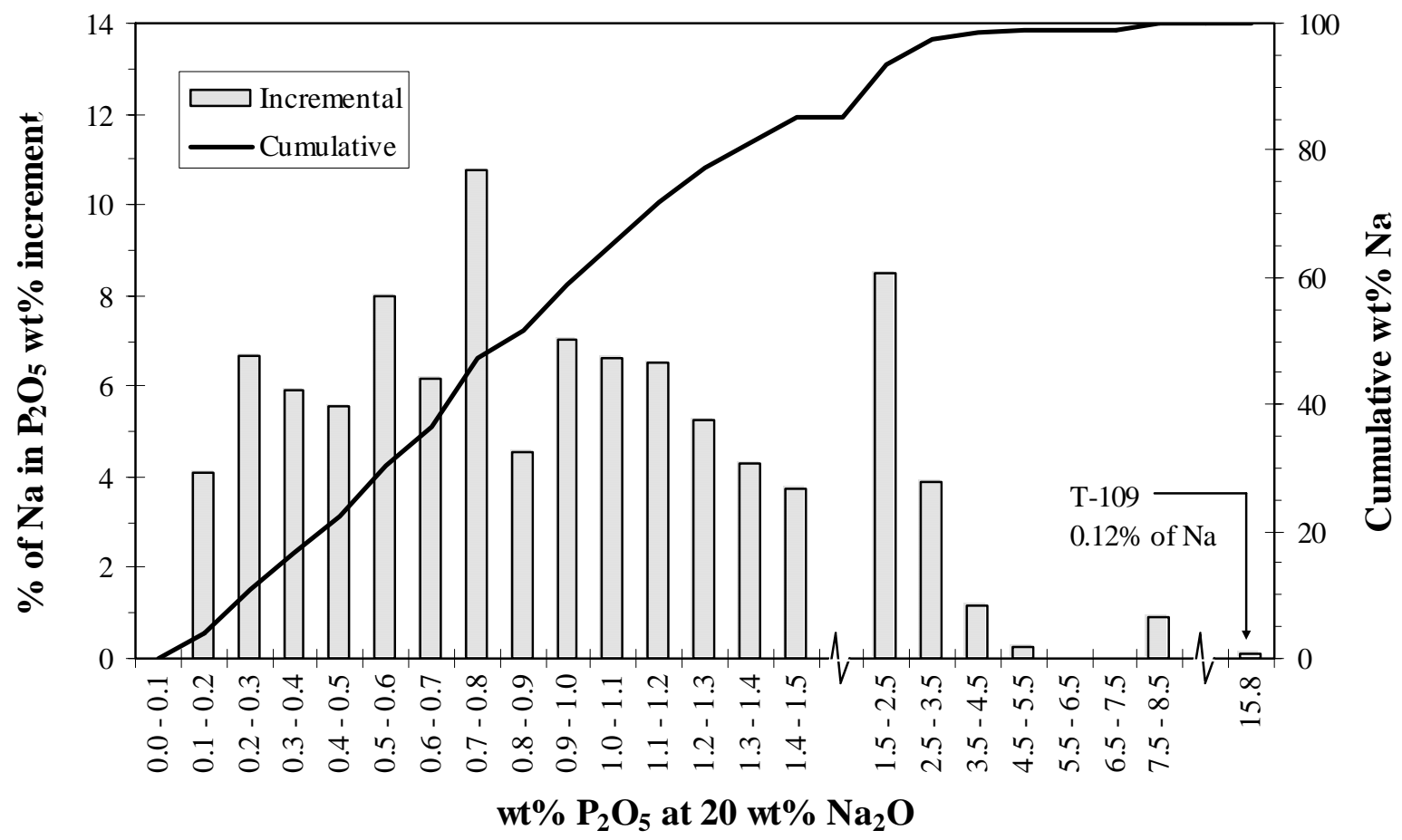

Figure 3.7. Relationship Between $\mathrm{P}_{2} \mathrm{O}_{5}$ and $\mathrm{Na}$ 


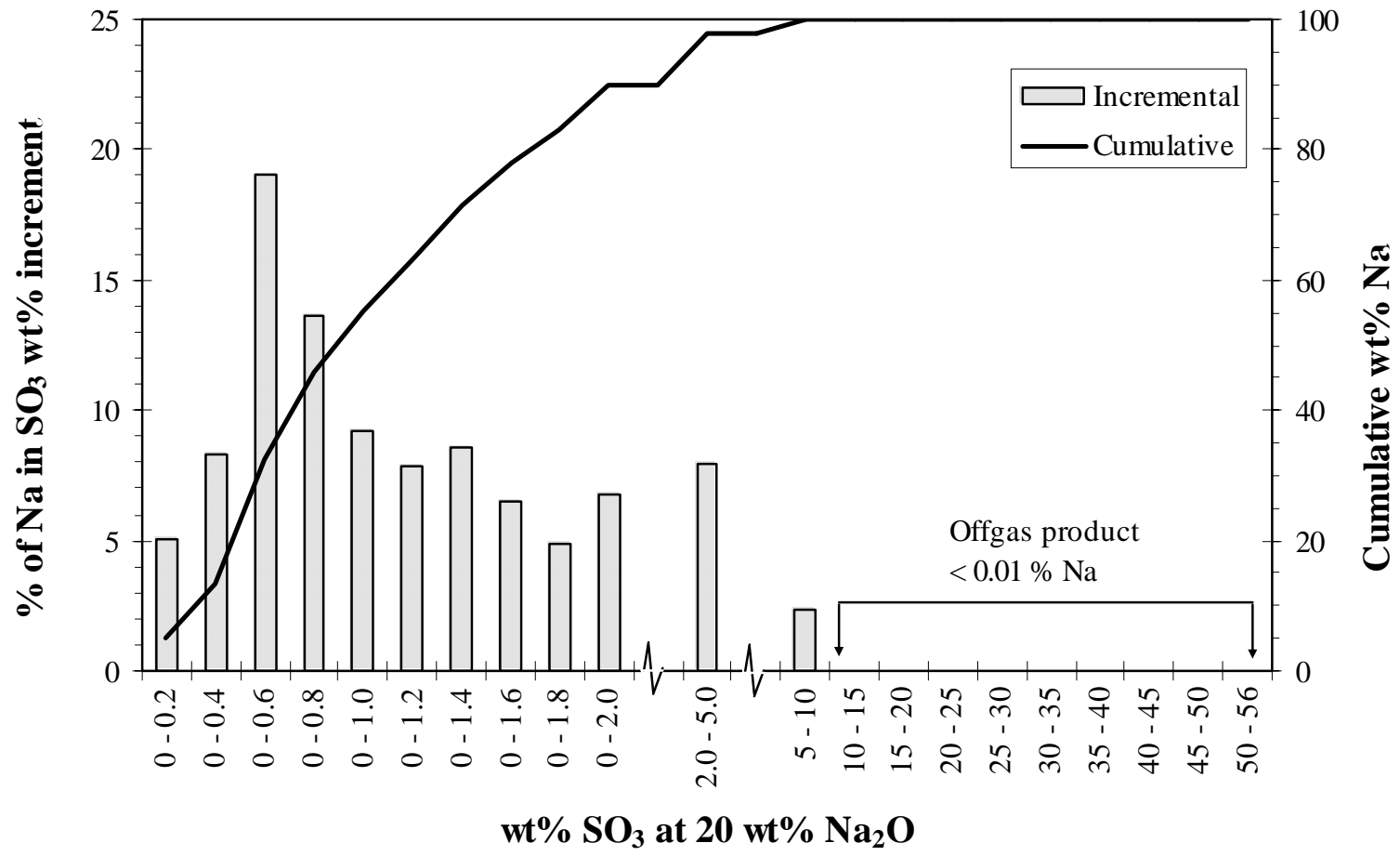

Figure 3.8. Relationship Between $\mathrm{SO}_{3}$ and $\mathrm{Na}$

Table 3.1. Summary of Significant Constituent Distributions

\begin{tabular}{|c|c|c|c|c|}
\hline Constituent & $\begin{array}{c}\text { Max. nominal } \\
\text { wt } \% \text { for } 90 \% \text { of } \\
\text { the } \mathrm{Na} \\
\end{array}$ & $\begin{array}{r}\text { Overall max. } \\
\text { nominal wt\% }\end{array}$ & $\begin{array}{c}\text { Max. nominal } \\
\text { wt\% in ICV tests } \\
\text { to date } \\
\end{array}$ & $\begin{array}{l}\% \text { of Na that is above } \\
\text { the tested maximum }\end{array}$ \\
\hline $\mathrm{Al}_{2} \mathrm{O}_{3}$ & 5.2 & 10.3 & 0.77 & 82 \\
\hline $\mathrm{CaO}$ & 0.024 & 0.25 & 0.0083 & 46 \\
\hline $\mathrm{Cl}$ & 0.40 & 8.6 & 0.20 & 62 \\
\hline $\mathrm{F}$ & 0.67 & 7.8 & 0.081 & 81 \\
\hline $\mathrm{MoO}_{3}$ & 0.0044 & 0.032 & --- & --- \\
\hline $\mathrm{NO}_{2}+\mathrm{NO}_{3}$ & 27 & 116 & 35.3 & 0.01 \\
\hline $\mathrm{P}_{2} \mathrm{O}_{5}$ & 1.7 & 16 & 0.50 & 78 \\
\hline $\mathrm{SO}_{3}$ & 2.0 & 56 & 0.99 & 45 \\
\hline
\end{tabular}


Some aspects of the constituent distributions shown in Figures 3.1 through 3.8 are summarized in Table 3.1, from which the following conclusions can be drawn:

- The maximum nominal concentrations of $\mathrm{MoO}_{3}$ and $\mathrm{CaO}$ are so low that although there may be reason to include these constituents in simulant recipes and glass formulations, there is no need to use them as criteria for defining "clusters."

- The maximum nominal concentrations of $\mathrm{CaO}$ and $\mathrm{SO}_{3}$ that, as of November 2004, have been tested in glasses are about equal to the Na-weighted averages for all streams (a little less than $50 \%$ of the $\mathrm{Na}$ is associated with higher concentrations).

- The maximum tested nominal concentration of $\mathrm{NO}_{2}$ plus $\mathrm{NO}_{3}$ is an upper bound for almost all the LAW-dominated streams.

- The concentrations of $\mathrm{Al}_{2} \mathrm{O}_{3}, \mathrm{Cl}, \mathrm{F}$, and $\mathrm{P}_{2} \mathrm{O}_{5}$ in most of the streams are well above the concentrations that have been tested in glasses.

Figures 3.9 though 3.14 show the nominal concentrations of $\mathrm{Al}_{2} \mathrm{O}_{3}, \mathrm{Cl}, \mathrm{F}, \mathrm{NO}_{2}+\mathrm{NO}_{3}, \mathrm{P}_{2} \mathrm{O}_{5}$, and $\mathrm{SO}_{3}$ in each of the nearly 1500 streams plotted against the mass of $\mathrm{Na}$ in each stream. These figures help to clarify which subsets of the feed streams contribute the significant species. The feed streams are subdivided into WTP streams that are dominated by off-gas-product, WTP streams that are dominated by LAW (which contain most of the Na), and streams derived from the 20 low-curie tank wastes. ${ }^{\text {(a) }}$ (Note that the streams dominated by off-gas product may contain some pretreated LAW waste; similarly, the LAW-dominated streams usually contain some off-gas product.) The LAW-dominated WTP streams are further subdivided into a general set and a high-F set, based on the grouping that is evident in Figure 3.11.

The conclusions to be drawn from Figures 3.9 through 3.14 include the following:

- The low-curie tank waste compositions are frequently outside the concentration ranges of most of the LAW streams generated by the WTP pre-treatment facility. Several low-curie wastes are higher in nominal $\mathrm{P}_{2} \mathrm{O}_{5}$ and $\mathrm{SO}_{3}$ than the WTP-derived LAW streams, and most are lower in nominal $\mathrm{Al}_{2} \mathrm{O}_{3}$ and $\mathrm{Cl}$.

- The streams dominated by off-gas product contain very little $\mathrm{Na}$ and $\mathrm{Al}$ but have high nominal concentrations of most of the significant anion species $\left(\mathrm{Cl}, \mathrm{F}, \mathrm{NO}_{2}+\mathrm{NO}_{3}\right.$, and $\left.\mathrm{SO}_{3}\right)$.

- The streams with high nominal fluoride (defined as those that are not dominated by off-gas product but have F concentration of $0.745 \mathrm{wt} \%$ or higher at $20 \mathrm{wt} \% \mathrm{Na}_{2} \mathrm{O}$ ) tend to be distinguishable from other LAW feed in other ways as well. Figures 3.9 and 3.14 show that the high-F LAW streams are lower than most in $\mathrm{Al}_{2} \mathrm{O}_{3}$ and $\mathrm{SO}_{3}$.

(a) In Figures 3.9 through 3.14, the low-curie tank waste streams stand out artificially from the WTP-based streams because they are considered as whole-tank batches and not broken down into weekly batches. They therefore contain much more Na per "batch." 


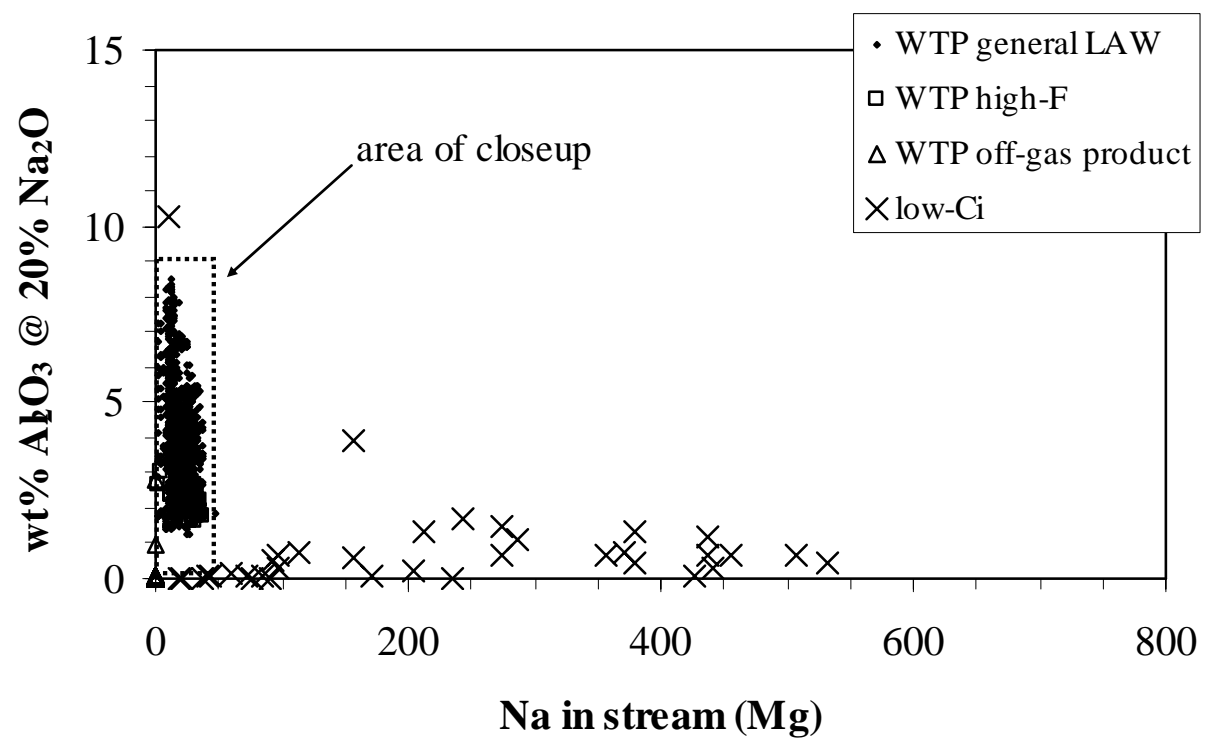

Figure 3.9a. Nominal $\mathrm{Al}_{2} \mathrm{O}_{3}$ Concentration Versus Na Mass, by Stream

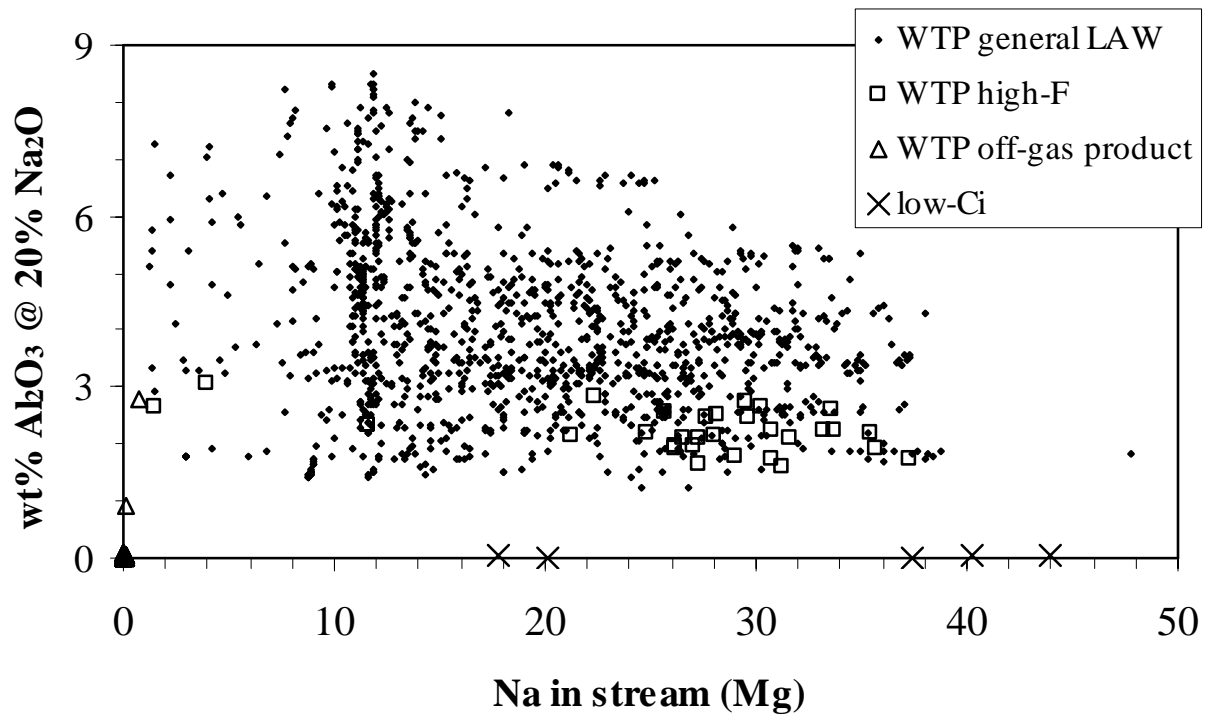

Figure 3.9b. Close-up of Nominal $\mathrm{Al}_{2} \mathrm{O}_{3}$ Versus Na Mass by Stream 


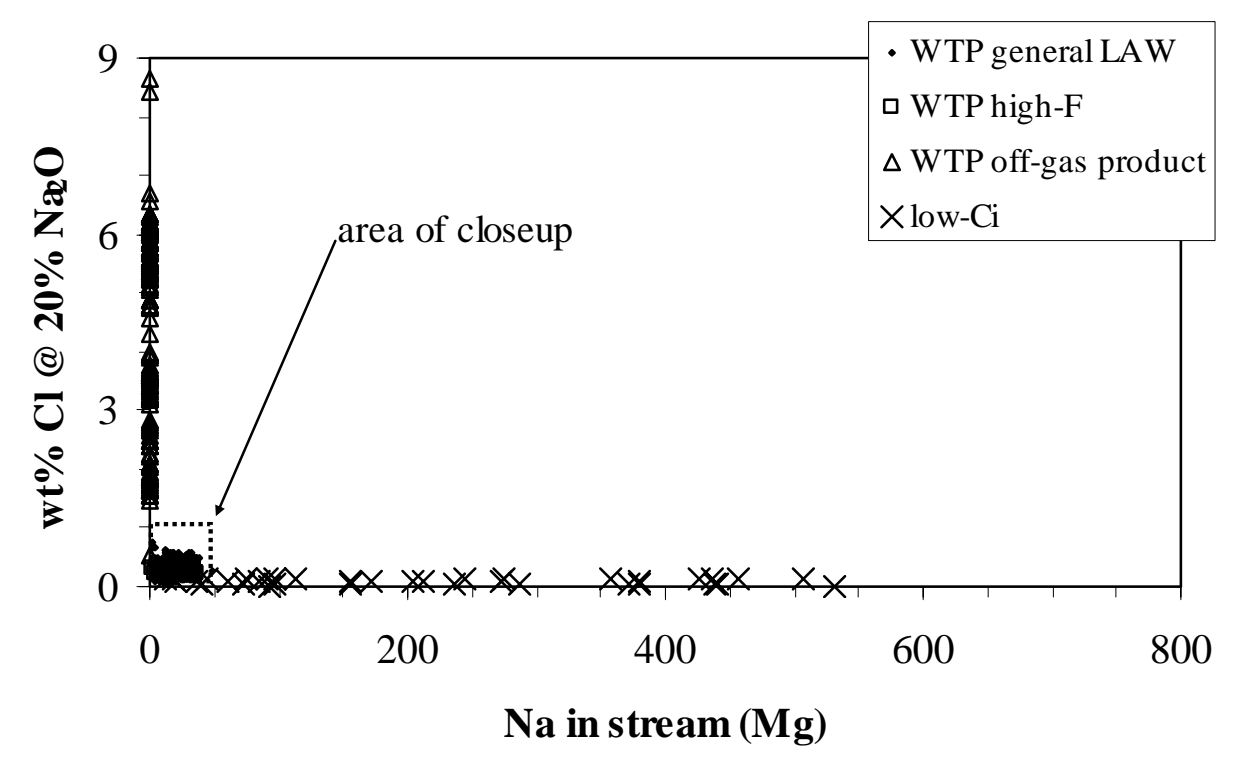

Figure 3.10a. Nominal Cl Concentration Versus Na Mass by Stream

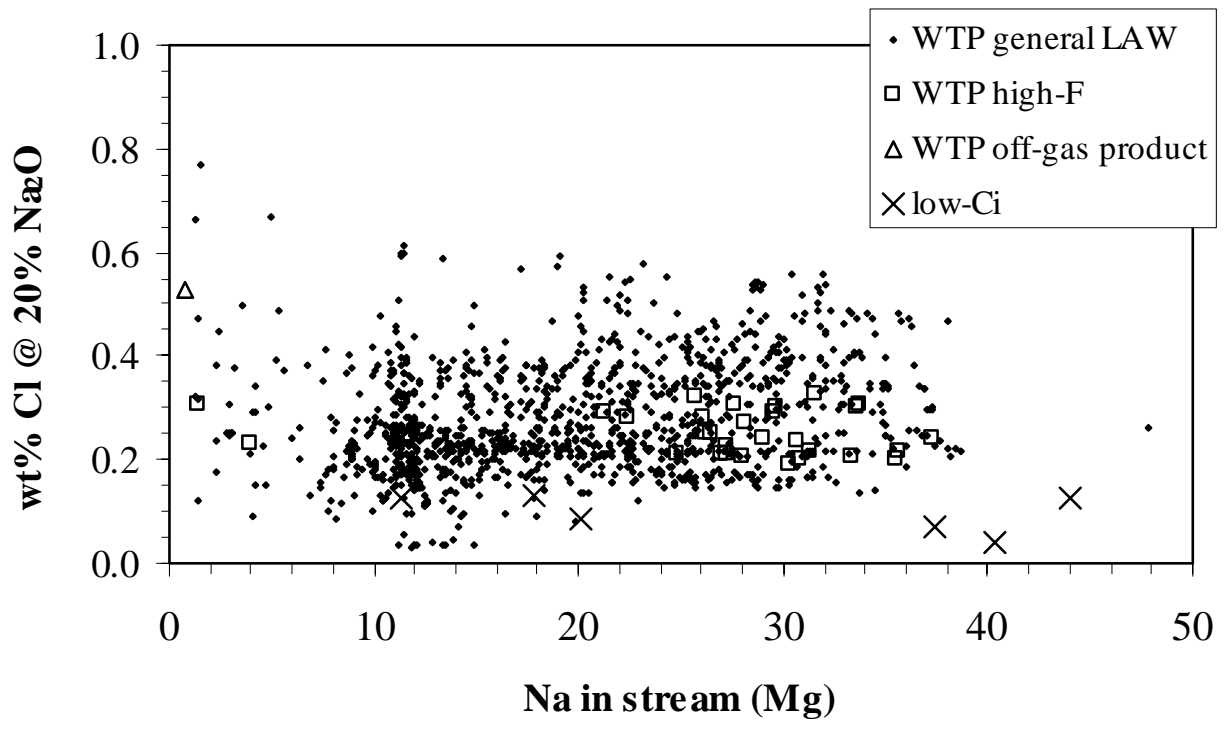

Figure 3.10b. Close-up of Nominal Cl Versus Na Mass by Stream 


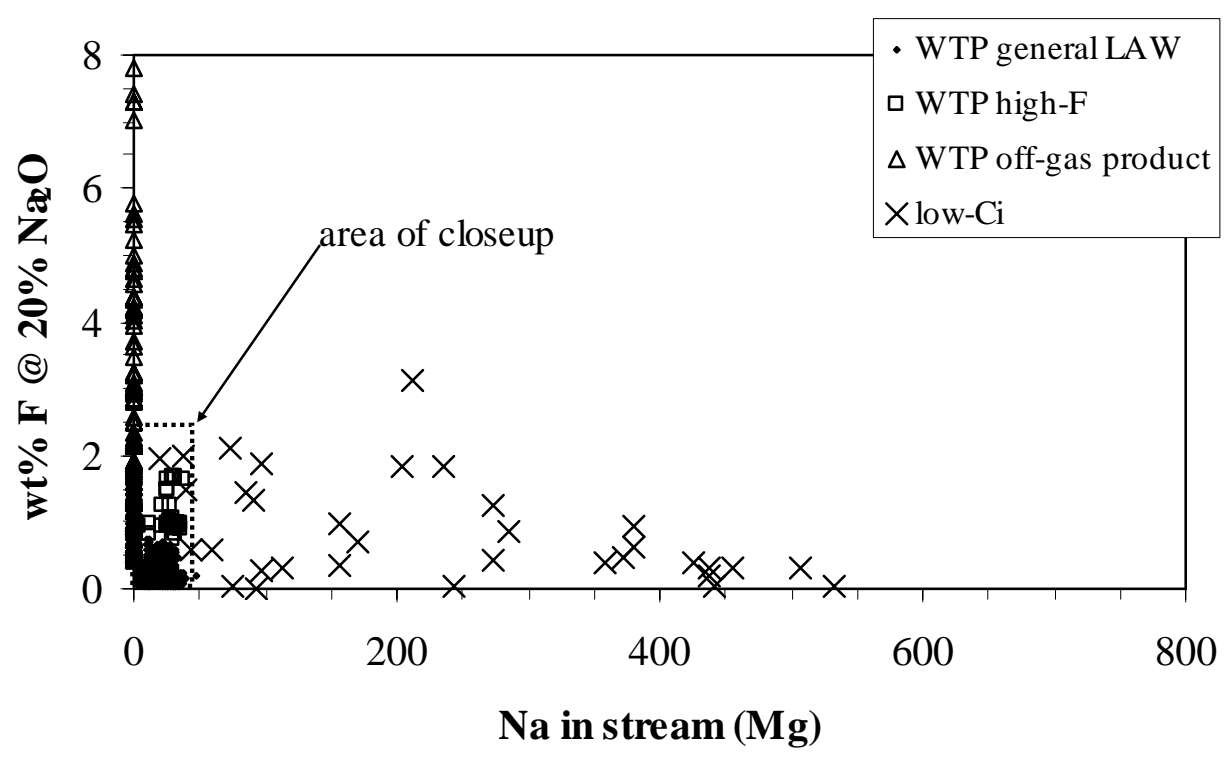

Figure 3.11a. Nominal F Concentration Versus Na Mass, by Stream

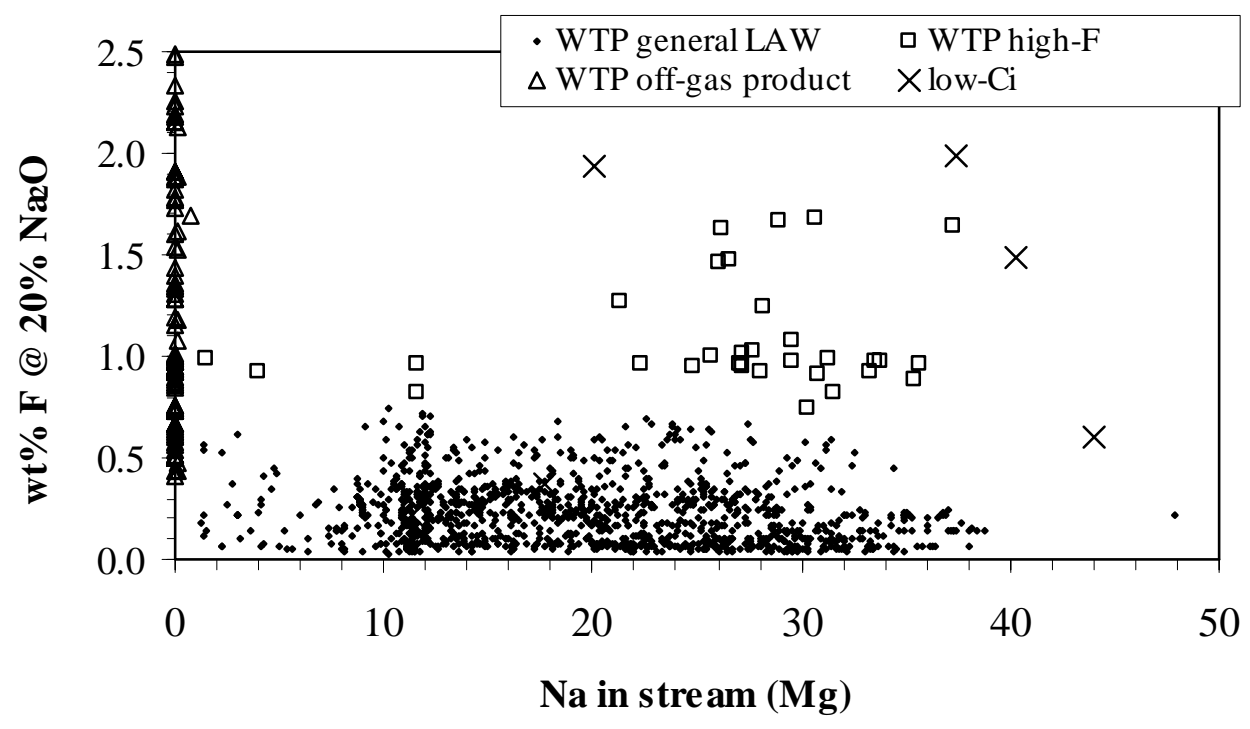

Figure 3.11b. Close-up of Nominal F Versus Na Mass, by Stream 


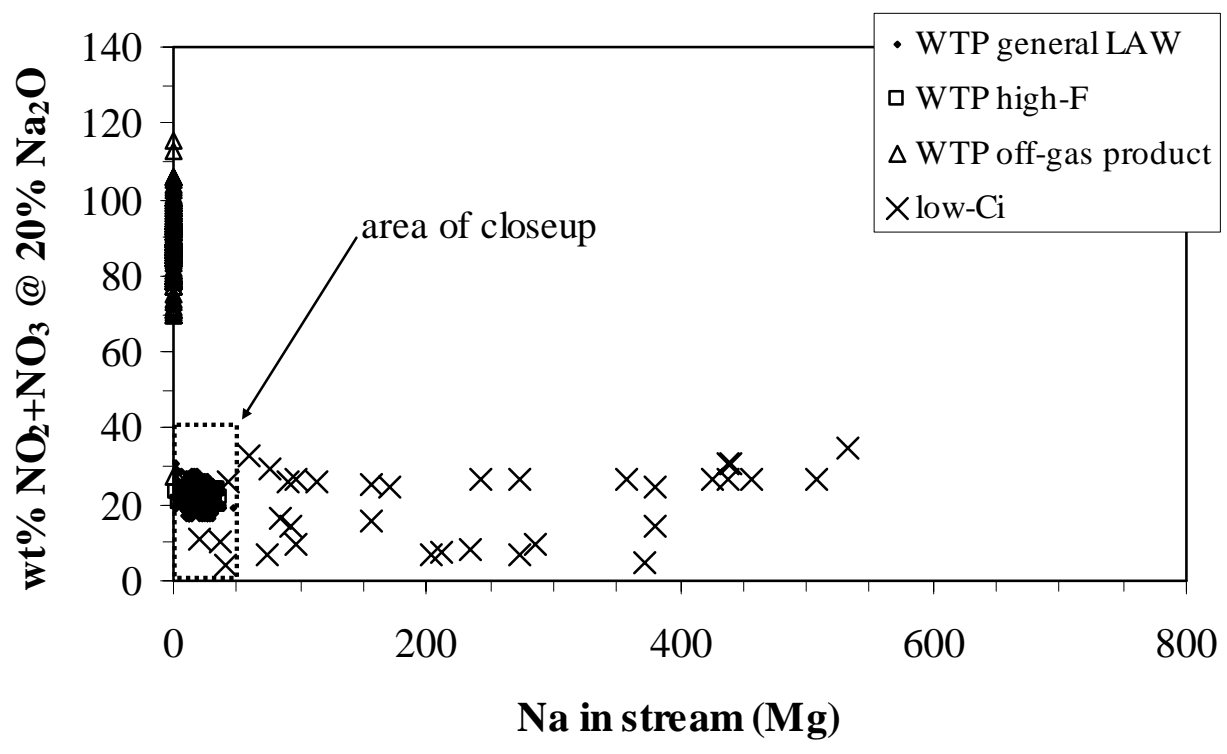

Figure 3.12a. Nominal $\left(\mathrm{NO}_{2}+\mathrm{NO}_{3}\right)$ Concentration Versus Na Mass by Stream

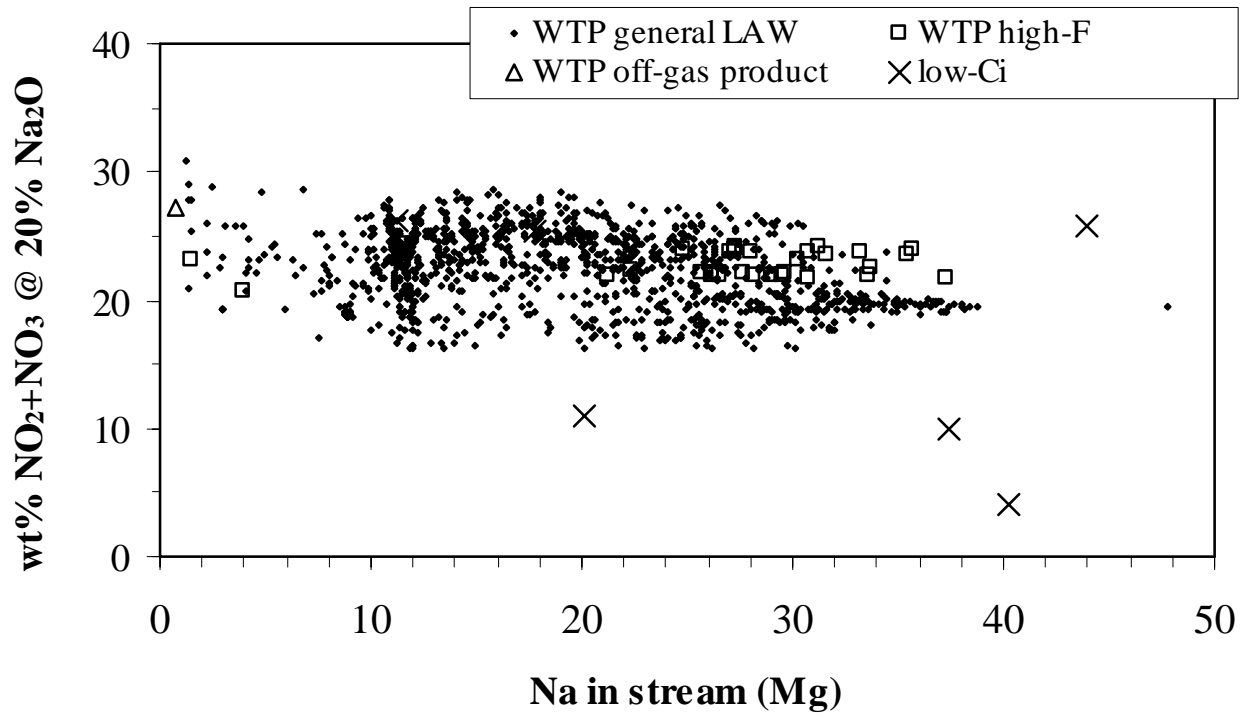

Figure 3.12b. Close-up of Nominal $\left(\mathrm{NO}_{2}+\mathrm{NO}_{3}\right)$ Versus Na Mass by Stream 


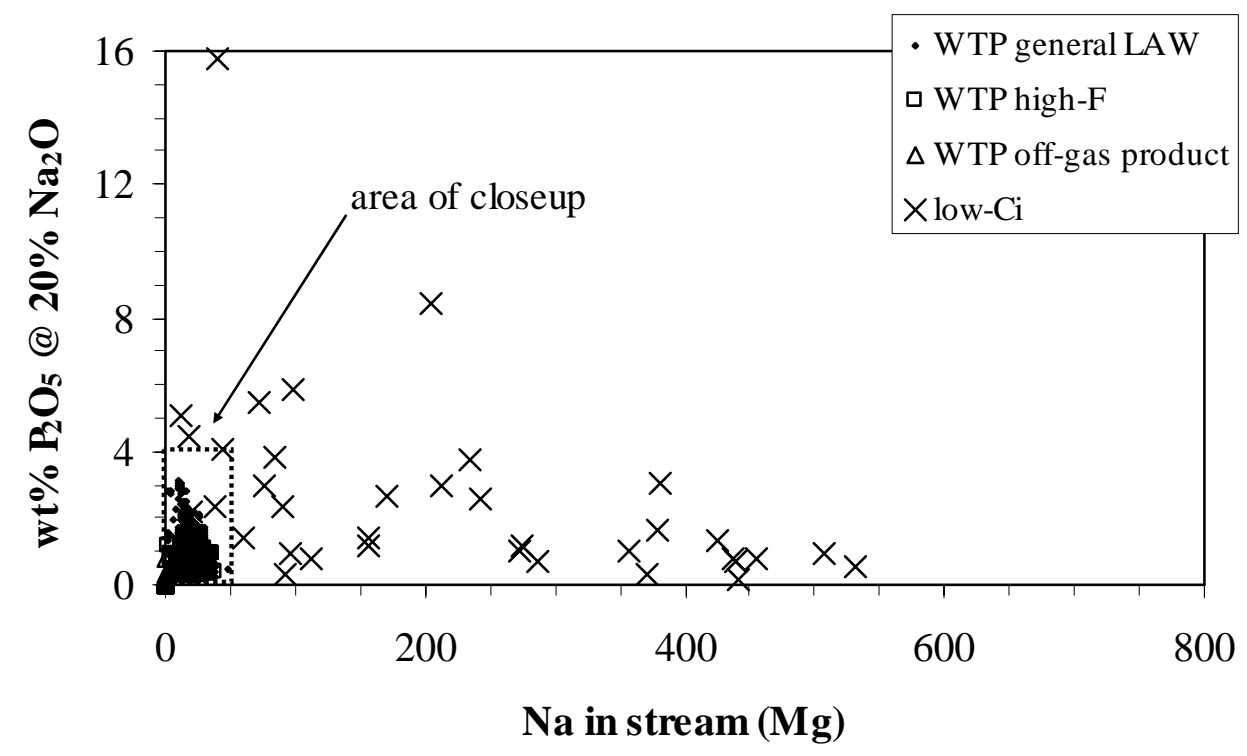

Figure 3.13a. Nominal $\mathrm{P}_{2} \mathrm{O}_{5}$ Concentration Versus Na Mass by Stream

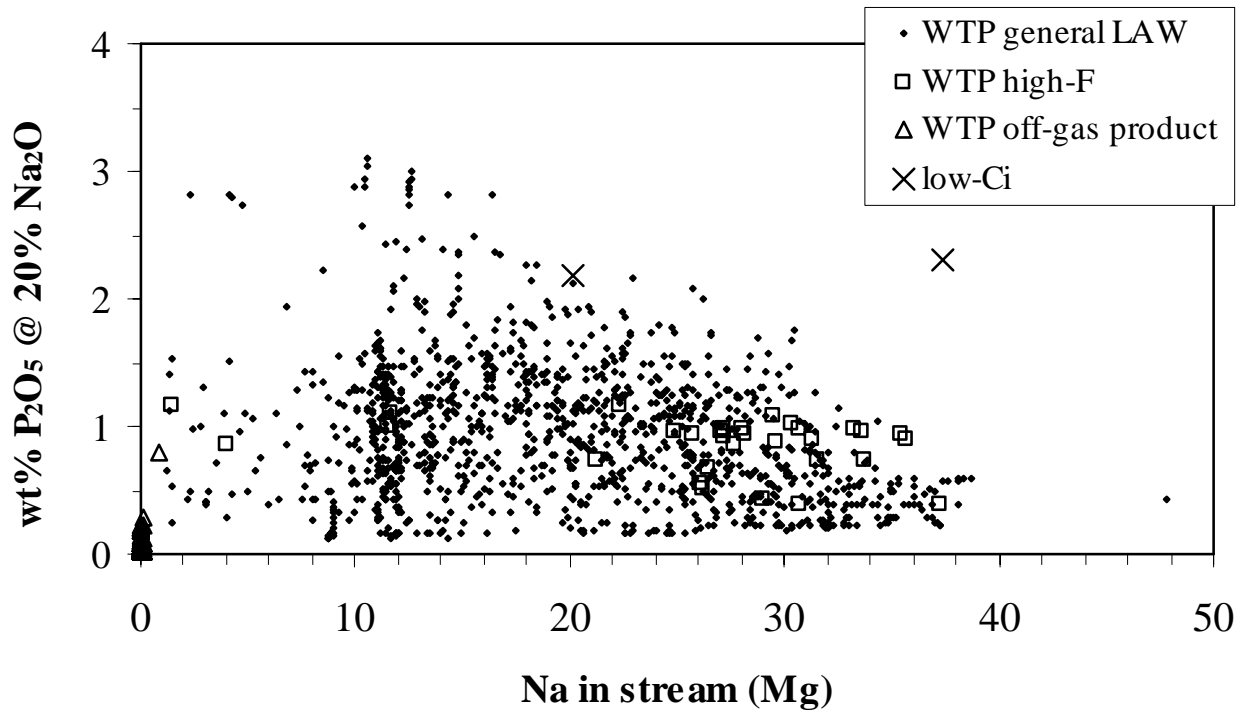

Figure 3.13b. Close-up of Nominal $\mathrm{P}_{2} \mathrm{O}_{5}$ Versus Na Mass by Stream 


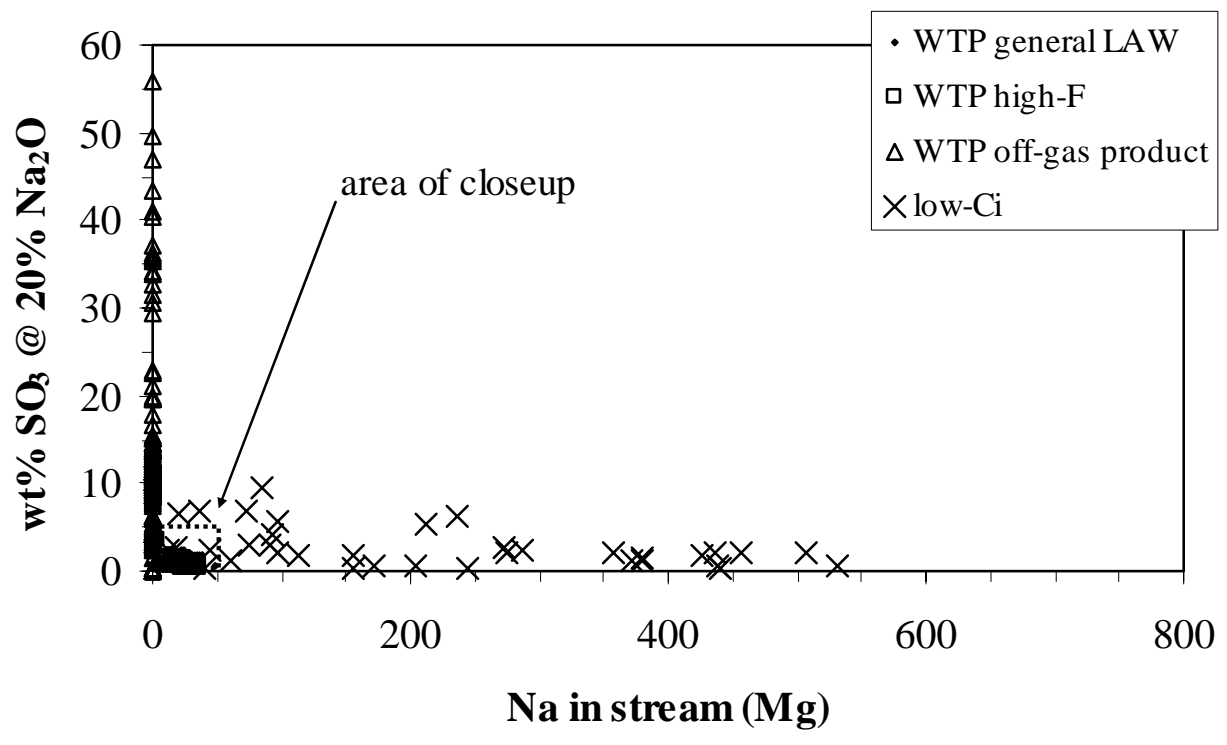

Figure 3.14a. Nominal $\mathrm{SO}_{3}$ Concentration Versus Na Mass, by Stream

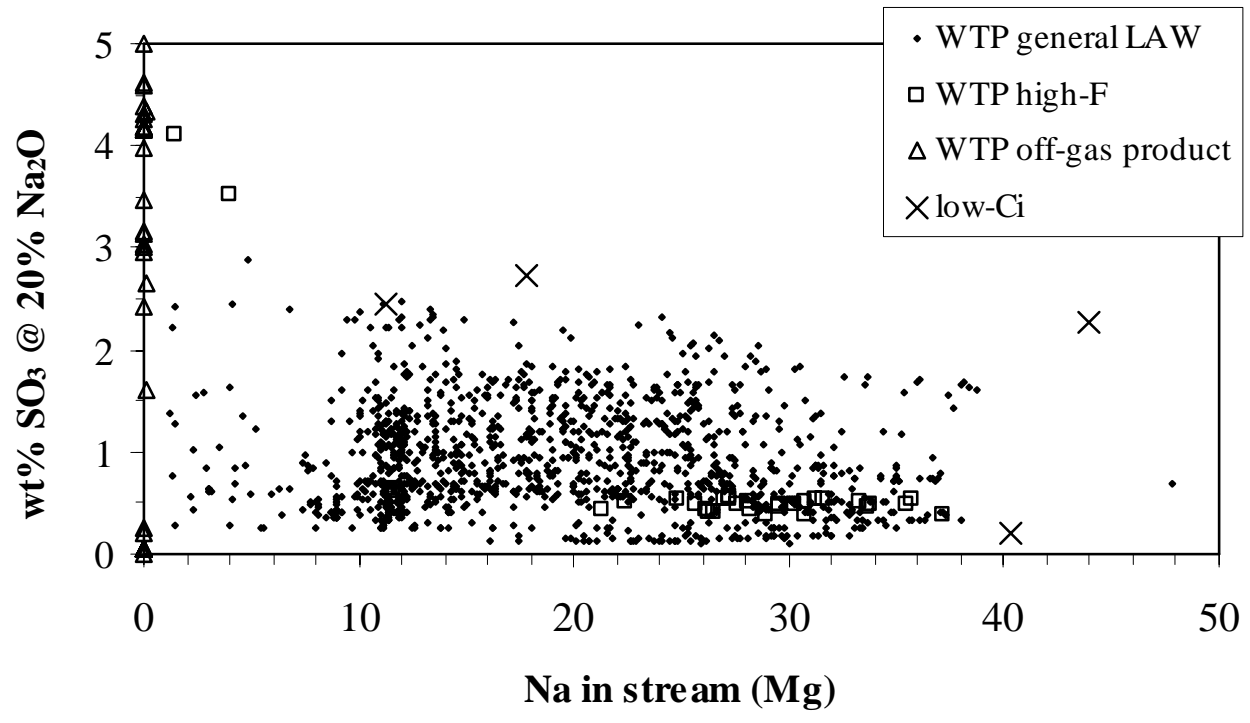

Figure 3.14b. Close-up of Nominal $\mathrm{SO}_{3}$ Versus Na Mass, by Stream 
PNNL-14985 Rev. 0

Page 22 of 78 


\subsection{Cluster Analysis}

The WTP-pretreated and low-curie-waste feed streams formed a large population of compositions that, for practical purposes, had to be reduced to roughly 20 potential waste formulations. The first step in accomplishing this was to separate the clearly distinguishable sets. These were

- Streams from low-curie tank wastes

- Streams dominated by off-gas product (defined as containing less than $1000 \mathrm{~kg} \mathrm{Na}$ )

- High-F WTP-pretreated LAW streams (defined as containing a nominal F concentration of 0.745 $w t \%$ or greater at $\left.20 \mathrm{wt} \% \mathrm{Na}_{2} \mathrm{O}\right) .{ }^{(\mathrm{a})}$ The fluoride breakpoint is low enough to have no significance to ICV. As was noted in Section 3 and shown in Figures 3.9 and 3.14, streams that contain more than this nominal F concentration, and streams that have less, tend to differ from each other in terms of some other constituent concentrations as well. This separation of populations was the reason for choosing the breakpoint.

The remaining majority of the WTP-derived streams were subjected to "cluster analysis," a statistical method for separating data points into groups. Each group contains the streams whose compositions are similar to the group's average composition and significantly different from the average compositions of the other groups. The computational tool used for this analysis was JMP ${ }^{\mathrm{TM}(\mathrm{b})}$ (SAS 2004a, 2004b). The statistical method of analysis was $k$-means clustering, an iterative approach to assigning data points to the nearest cluster.

To perform a cluster analysis with the JMP program, the user inputs the variables to use as clusterdefinition criteria and the desired number of clusters. The nominal concentrations of $\mathrm{Al}_{2} \mathrm{O}_{3}, \mathrm{P}_{2} \mathrm{O}_{5}$, and $\mathrm{SO}_{3}$ (that is, the concentrations of those oxides at a nominal $\mathrm{Na}_{2} \mathrm{O}$ concentration of $20 \mathrm{wt} \%$ ) were chosen as cluster criteria. To prevent the higher concentration range of $\mathrm{Al}_{2} \mathrm{O}_{3}$ from overly influencing the cluster selection in its favor, the three concentrations were standardized by dividing them by their standard deviations before they were used to define clusters. Different numbers of clusters were tested and it was found that a minimum of 15 clusters were needed to produce distinct clusters with relatively narrow ranges of the criterion variables. Note that all of these 15 clusters are exactly the same as in the preceding Series-22 report. $^{\left({ }^{(c)}\right.}$

No close matches were found between the 36 low-curie tank waste streams and the 15 WTP-derived LAW clusters. By contrast, the Series-22 report found that the five BY tanks in the excluded low-curie waste set matched WTP-derived LAW clusters. Apparently the application of selective dissolution factors decreased the similarity between low-curie tank wastes and WTP-derived LAW.

(a) One borderline high-fluoride stream, the one predicted by G2 for the week of June 9, 2032, was placed in a lowfluoride cluster (number 14). This stream assignment did not significantly skew the composition of either of the clusters involved because the stream contained only $1 \%$ of the total cluster Na.

(b) JMP is a trademark of SAS Institute, Inc., Cary, North Carolina.

(c) Mahoney, L.A. November 12, 2004. Waste Simulant Formulation for Series-22 Bulk Vitrification Tests, letter report ST05.004, Pacific Northwest National Laboratory, Richland, Washington. 
Because the current study found no matches between individual low-curie waste streams and WTPderived clusters, the low-curie waste streams were put through cluster analysis separate from the one done for WTP-derived LAW. This approach also allowed the WTP-derived streams and the low-curie-waste streams to be maintained in separate categories, which is desirable because different blending and pretreatment assumptions were made for these groups. The low-curie cluster analysis separated the lowcurie waste streams into 8 distinct clusters that were broader than the clusters for the WTP-derived LAW streams. These 8 clusters are replacements for the 4 low-curie clusters in the Series-22 report.

Table 4.1 gives the characteristics of the final 25 clusters in terms of the nominal concentrations of $\mathrm{Al}_{2} \mathrm{O}_{3}, \mathrm{P}_{2} \mathrm{O}_{5}$, and $\mathrm{SO}_{3}$ (in 20 wt $\% \mathrm{Na}_{2} \mathrm{O}$ glass) and the mass of $\mathrm{Na}$ in each cluster. The "O," "F," and "Yn" clusters in Table 4.1 represent, respectively, off-gas product, high-F WTP-pretreated LAW, and low-curie tank wastes. This version of Table 4.1 should be used instead of Table 2 in the Series-22 report because it includes a larger set of low-curie wastes.. The current table has different low-curie waste clusters; in addition, the Na totals are different for some of the WTP-derived clusters because the Na contributed by the BY low-curie tanks has been removed.

Figures 4.1 through 4.3 depict the distributions of the nominal $\mathrm{Al}_{2} \mathrm{O}_{3}, \mathrm{P}_{2} \mathrm{O}_{5}$, and $\mathrm{SO}_{3}$ concentrations in the clusters and in the total ST feed (labeled "ST"). The clusters are ranged along the $\mathrm{x}$ axis, and the nominal concentration is on the y axis. In the symbol used for each cluster, the box represents the range from the arithmetic average minus one standard deviation to the arithmetic average plus one standard deviation. The top line of the symbol ends at the maximum concentration, and the bottom line ends at the minimum. The diamonds represent the Na-weighted average nominal concentration, which is in most cases lower than the arithmetic average. Clusters that contain only one stream are shown by dashes, which in some cases are off scale and therefore marked with arrows. 
PNNL-14985 Rev. 0

Table 4.1. Cluster Definitions

\begin{tabular}{|c|c|c|c|c|c|c|c|c|c|c|}
\hline \multirow[b]{3}{*}{ Cluster } & \multirow{3}{*}{$\begin{array}{c}\text { Mass } \\
\text { of } \mathrm{Na} \\
\text { (Mg) }\end{array}$} & \multicolumn{9}{|c|}{ "Nominal wt\% ${ }^{\text {(a) }}$} \\
\hline & & \multicolumn{3}{|c|}{$\mathrm{Al}_{2} \mathrm{O}_{3}$} & \multicolumn{3}{|c|}{$\mathbf{P}_{2} \mathbf{O}_{5}$} & \multicolumn{3}{|c|}{$\mathrm{SO}_{3}$} \\
\hline & & Min. & Avg. & Max. & Min. & Avg. & Max. & Min. & Avg. & Max. \\
\hline 1 & 6059 & 3.6 & 4.5 & 5.9 & 0.16 & 0.51 & 0.93 & 0.11 & 0.42 & 0.78 \\
\hline 2 & 1314 & 2.6 & 3.5 & 5.1 & 1.4 & 1.8 & 2.4 & 0.60 & 0.86 & 1.2 \\
\hline 3 & 1715 & 3.5 & 4.4 & 5.8 & 0.89 & 1.2 & 1.6 & 0.97 & 1.3 & 1.7 \\
\hline 4 & 273 & 4.7 & 5.9 & 6.6 & 2.2 & 2.7 & 3.1 & 0.45 & 0.66 & 1.0 \\
\hline 5 & 14 & 4.6 & 4.8 & 5.8 & 1.4 & 1.6 & 1.6 & 1.9 & 2.0 & 2.4 \\
\hline 6 & 2169 & 2.3 & 3.3 & 4.1 & 0.88 & 1.1 & 1.5 & 1.2 & 1.6 & 2.0 \\
\hline 7 & 2571 & 2.0 & 3.2 & 4.4 & 0.58 & 0.98 & 1.4 & 0.41 & 0.83 & 1.2 \\
\hline 8 & 1467 & 1.7 & 2.5 & 3.4 & 0.25 & 0.70 & 1.0 & 1.0 & 1.4 & 1.8 \\
\hline 9 & 184 & 2.2 & 2.7 & 3.1 & 0.16 & 0.18 & 0.28 & 1.8 & 2.2 & 2.5 \\
\hline 10 & 1019 & 6.0 & 7.1 & 8.5 & 0.16 & 0.44 & 1.0 & 0.13 & 0.38 & 0.88 \\
\hline 11 & 1089 & 4.9 & 6.0 & 7.5 & 0.53 & 0.88 & 1.5 & 0.71 & 1.0 & 1.6 \\
\hline 12 & 1478 & 1.5 & 2.4 & 3.3 & 1.2 & 1.5 & 2.0 & 0.82 & 1.2 & 1.7 \\
\hline 13 & 958 & 1.2 & 2.0 & 3.2 & 0.86 & 1.2 & 1.5 & 1.8 & 2.0 & 2.9 \\
\hline 14 & 740 & 3.9 & 4.9 & 6.9 & 0.89 & 1.4 & 1.9 & 0.33 & 0.70 & 1.1 \\
\hline 15 & 2956 & 1.4 & 2.7 & 3.6 & 0.12 & 0.35 & 0.77 & 0.35 & 0.60 & 0.98 \\
\hline $\mathrm{O}$ & 4.0 & 0.011 & 0.62 & 2.8 & 0.017 & 0.22 & 0.79 & 0.003 & 12 & 56 \\
\hline$F$ & 819 & 1.6 & 2.2 & 3.0 & 0.39 & 0.84 & 1.2 & 0.39 & 0.50 & 4.1 \\
\hline$Y 1^{(b)}$ & 40 & 0.067 & 0.067 & 0.067 & 16 & 16 & 16 & 0.22 & 0.22 & 0.22 \\
\hline $\mathrm{Y}^{(\mathrm{c})}$ & 11 & 10 & 10 & 10 & 5.1 & 5.1 & 5.1 & 2.4 & 2.4 & 2.4 \\
\hline $\mathrm{Y3}^{(\mathrm{d})}$ & 1186 & 0.030 & 1.5 & 3.9 & 0.73 & 1.2 & 2.4 & 1.2 & 2.0 & 3.0 \\
\hline $\mathrm{Y} 4^{(\mathrm{e})}$ & 212 & 1.3 & 1.3 & 1.3 & 3.0 & 3.0 & 3.0 & 5.4 & 5.4 & 5.4 \\
\hline$Y 5^{(\mathrm{f})}$ & 5691 & 0.031 & 0.61 & 1.7 & 0.16 & 1.1 & 4.4 & 0.17 & 1.4 & 4.1 \\
\hline$Y 6^{(\mathrm{g})}$ & 85 & 0.038 & 0.038 & 0.038 & 3.8 & 3.8 & 3.8 & 9.6 & 9.6 & 9.6 \\
\hline$Y 7^{(\mathrm{h})}$ & 204 & 0.2 & 0.2 & 0.2 & 8.5 & 8.5 & 8.5 & 0.48 & 0.48 & 0.48 \\
\hline$Y 8^{(i)}$ & 463 & 0 & 0.075 & 0.32 & 2.2 & 4.3 & 5.9 & 5.6 & 6.2 & 6.8 \\
\hline ST Feed $^{(j)}$ & 27998 & 0 & 3.4 & 10 & 0.017 & 0.96 & 5.9 & 0.003 & 1.1 & 56 \\
\hline Overall $^{(\mathrm{k})}$ & 32723 & 0 & 3.0 & 10 & 0.017 & 1.1 & 16 & 0.003 & 1.2 & 56 \\
\hline
\end{tabular}

(a) "Nominal" indicates that concentrations are based on an assumed $20 \mathrm{wt} \% \mathrm{Na}_{2} \mathrm{O}$, though some compositions would contain more or less than that amount of soda in practice.

(b) Cluster Y1 contains T-109 and is 100\% double-counted.

(c) Cluster Y2 contains BX-103 and is 0\% double-counted.

(d) Cluster Y3 contains BX-107, BY-102, BY-103, BY-108, and BY-111. The Na in the cluster is 32.0\% doublecounted (from BY-103).

(e) Cluster Y4 contains BY-109 and is 100\% double-counted.

(f) Cluster Y5 contains B-101, B-106, BX-108, BX-109, BX-110, BX-111, BY-105, BY-112, S-105, S-109, TX103, TX-105, TX-108, TX-110, TX-111, TX-112, TX-114, TX-115, TX-117, TY-102, and U-107. The Na in the cluster is $68.3 \%$ double-counted. It should be noted that $\mathrm{S}-109$ waste is not conservatively representative of this cluster, being below average in all three criterion constituents (nominal $\mathrm{Al}_{2} \mathrm{O}_{3}=0.48 \%, \mathrm{P}_{2} \mathrm{O}_{5}=0.51 \%$,

$\left.\mathrm{SO}_{3}=0.52 \%\right)$.

(g) Cluster Y6 contains B-107 and is 0\% double-counted.

(h) Cluster Y7 contains TX-118 and is 100\% double-counted.

(i) Cluster Y8 contains B-102, B-103, B-105, B-108, and B-109. The cluster is 0\% double-counted.

(j) The ST feed contains all WTP-derived streams plus the 20 excluded low-curie SST wastes. It does not include the 16 double-counted low-curie wastes.

(k) “Overall” contains all the streams, ST feed plus the 16 double-counted low-curie SST wastes. 


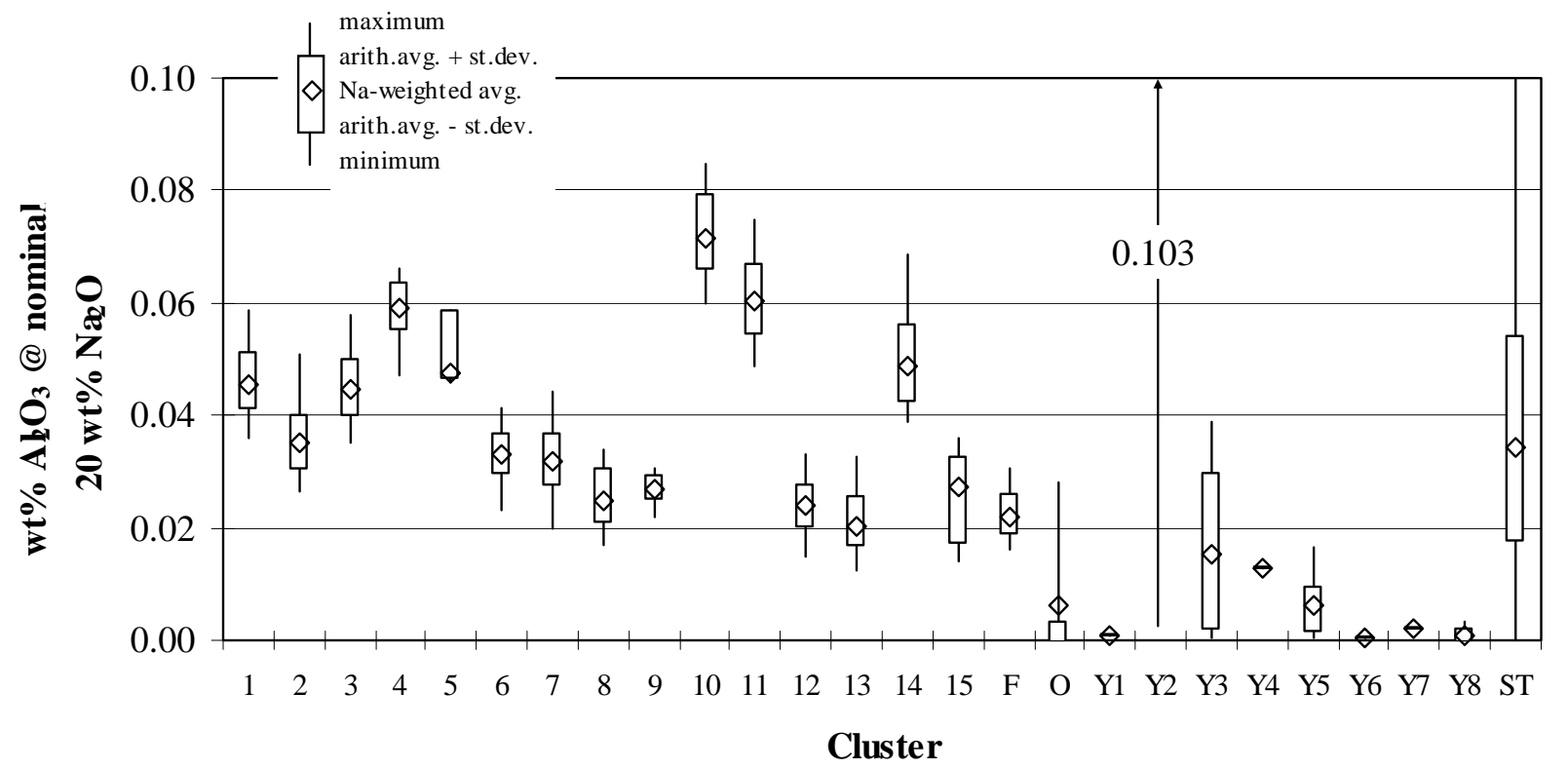

Figure 4.1. Distribution of Nominal $\mathrm{Al}_{2} \mathrm{O}_{3}$ Concentrations Among Clusters

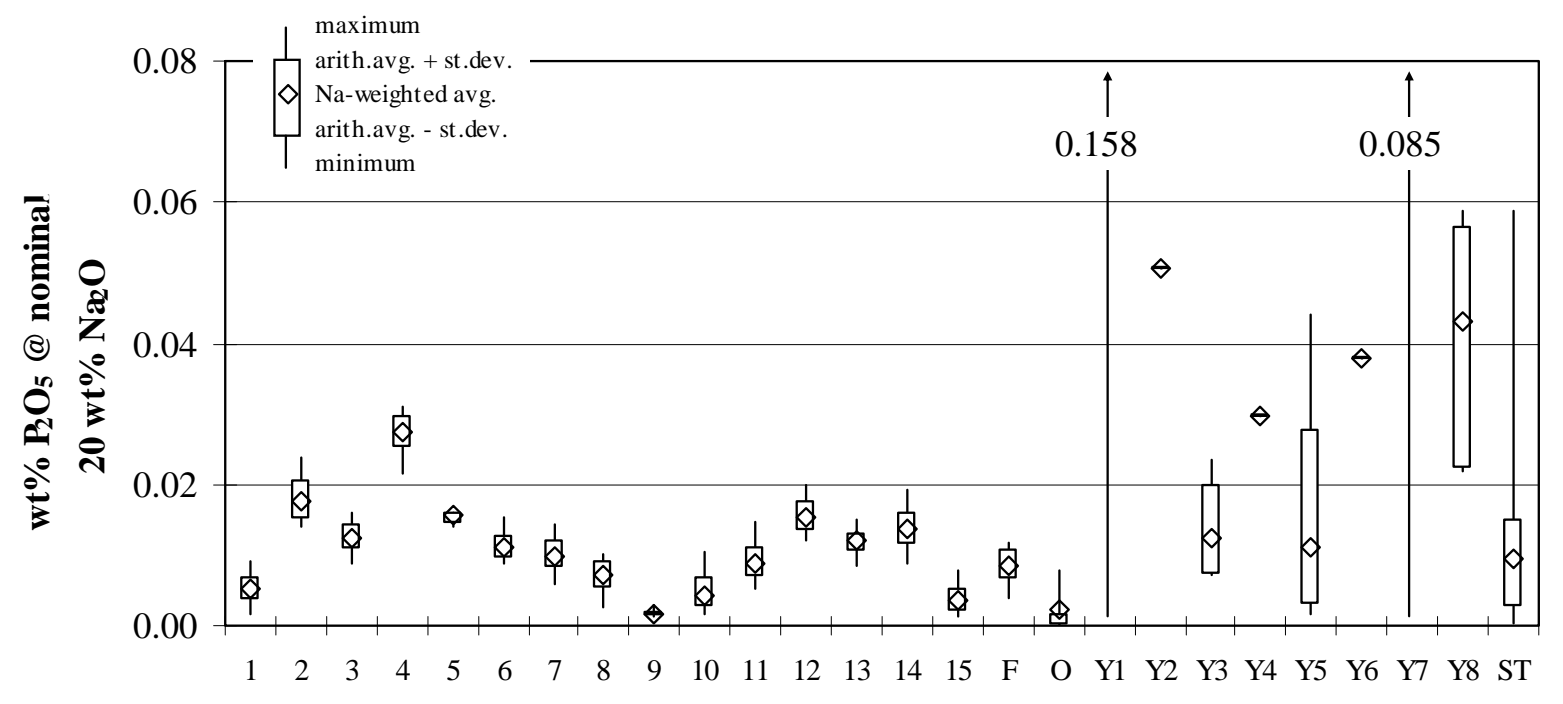

Cluster

Figure 4.2. Distribution of Nominal $\mathrm{P}_{2} \mathrm{O}_{5}$ Concentrations Among Clusters 


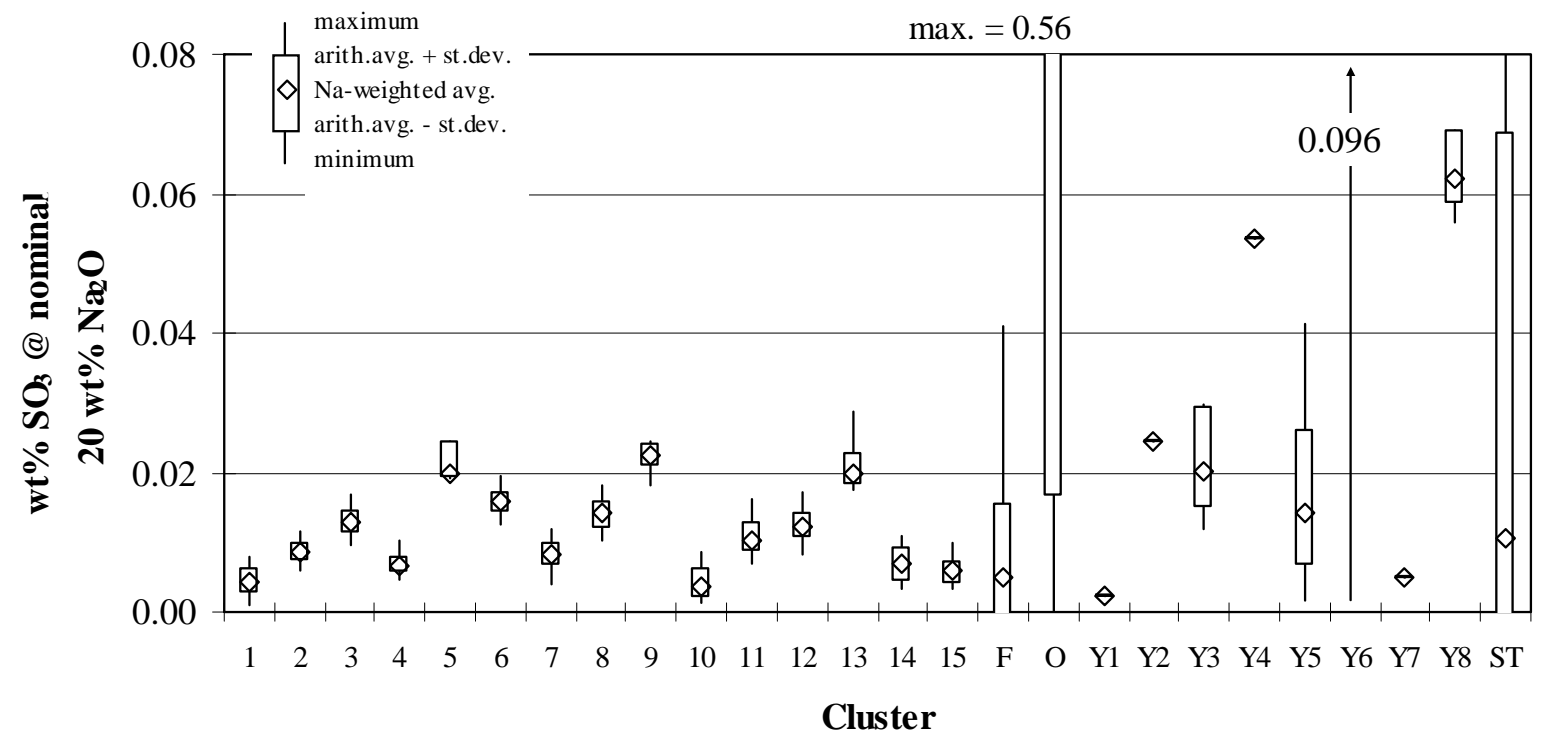

Figure 4.3. Distribution of Nominal $\mathrm{SO}_{3}$ Concentrations Among Clusters 
PNNL-14985 Rev. 0

Page 28 of 78 


\subsection{Cluster Compositions}

The compositions of the 25 clusters already described were calculated in terms of the cluster-average waste oxide fraction of every constituent for which data were available. Equation (5.1) was used to find the waste-oxide fraction.

$$
x_{i}=\frac{m_{i} f_{i}}{\sum_{j} m_{j} f_{j}}
$$

where $\quad \mathrm{x}_{\mathrm{i}}=$ weight fraction of oxide $i$ in waste-oxide mixture

$\mathrm{m}_{\mathrm{i}}=$ mass of species $i$ in stream

$\mathrm{f}_{\mathrm{i}}=$ stoichiometric factor that converts species $i$ to its oxide form; this factor is 0 for $\mathrm{NO}_{2}$, $\mathrm{NO}_{3}, \mathrm{NH}_{3}, \mathrm{OH}$, organic and inorganic carbon species, water, and other species with volatile products.

The resulting waste-oxide compositions, which are the compositions needed for glass formulation assessment, are given in Tables 5.1 through 5.25. Of these, Tables 5.1 through 5.17 are identical to Tables 3 through 19 in the Series-22 report. Tables 5.18 through 5.25 differ from Tables 20 through 23 in the Series 22 report and should be used instead of them.

A later report, supporting the Series 33 tests, will provide full recipes for the Series 33 simulants in terms of the sodium salts and other compounds found in the waste. Charge-balanced compositions will be provided as part of that task. 
Table 5.1. Cluster 1 Waste Oxide Composition

\begin{tabular}{|c|c|c|c|c|c|}
\hline $\begin{array}{c}\text { Radioactive } \\
\text { oxides }\end{array}$ & $\begin{array}{l}\text { Wt. } \\
\text { frac. }\end{array}$ & $\begin{array}{l}\text { Supplemental } \\
\text { Species Oxides }\end{array}$ & $\begin{array}{l}\text { Wt. } \\
\text { frac. }\end{array}$ & $\begin{array}{c}\text { BBI Species } \\
\text { Oxides }\end{array}$ & Wt. frac. \\
\hline${ }^{106} \mathrm{RuO}_{2}$ & $3.4 \mathrm{E}-18$ & $\mathrm{Ag}_{2} \mathrm{O}$ & $8.3 \mathrm{E}-06$ & $\mathrm{Al}_{2} \mathrm{O}_{3}$ & 0.169 \\
\hline${ }^{113} \mathrm{CdO}$ & $1.2 \mathrm{E}-10$ & $\mathrm{As}_{2} \mathrm{O}_{5}$ & 3.9E-05 & $\mathrm{Bi}_{2} \mathrm{O}_{3}$ & $6.4 \mathrm{E}-05$ \\
\hline${ }^{12} 5 \mathrm{Sb}_{2} \mathrm{O}_{3}$ & $1.7 \mathrm{E}-12$ & $\mathrm{~B}_{2} \mathrm{O}_{3}$ & $1.7 \mathrm{E}-04$ & $\mathrm{CaO}$ & 0.00034 \\
\hline${ }^{126} \mathrm{SnO}_{2}$ & 2.7E-07 & $\mathrm{BaO}$ & 8.8E-06 & $\mathrm{Cl}$ & 0.015 \\
\hline${ }^{129} \mathrm{I}$ & $5.7 \mathrm{E}-06$ & $\mathrm{BeO}$ & $3.7 \mathrm{E}-06$ & $\mathrm{Cr}_{2} \mathrm{O}_{3}$ & 0.0069 \\
\hline${ }^{134} \mathrm{Cs}_{2} \mathrm{O}$ & 4.9E-17 & $\mathrm{CdO}$ & 5.4E-06 & $\mathrm{F}$ & 0.0042 \\
\hline${ }^{137} \mathrm{Cs}_{2} \mathrm{O}$ & $9.9 \mathrm{E}-10$ & $\mathrm{Ce}_{2} \mathrm{O}_{3}$ & 8.1E-04 & $\mathrm{Fe}_{2} \mathrm{O}_{3}$ & 0.00023 \\
\hline${ }^{137} \mathrm{BaO}$ & 8.3E-13 & $\mathrm{Co}_{2} \mathrm{O}_{3}$ & 6.6E-06 & $\mathrm{K}_{2} \mathrm{O}$ & 0.018 \\
\hline${ }^{151} \mathrm{Sm}_{2} \mathrm{O}_{3}$ & $3.2 \mathrm{E}-07$ & $\mathrm{Cs}_{2} \mathrm{O}$ & $3.9 \mathrm{E}-09$ & $\mathrm{La}_{2} \mathrm{O}_{3}$ & $7.4 \mathrm{E}-06$ \\
\hline${ }^{152} \mathrm{Eu}_{2} \mathrm{O}_{3}$ & $1.6 \mathrm{E}-11$ & $\mathrm{CuO}$ & 5.9E-06 & $\mathrm{MnO}$ & $2.1 \mathrm{E}-05$ \\
\hline${ }^{154} \mathrm{Eu}_{2} \mathrm{O}_{3}$ & $1.6 \mathrm{E}-10$ & $\mathrm{Li}_{2} \mathrm{O}$ & $1.3 \mathrm{E}-05$ & $\mathrm{Na}_{2} \mathrm{O}$ & 0.741 \\
\hline${ }^{15} 5 \mathrm{Eu}_{2} \mathrm{O}_{3}$ & 2.4E-11 & $\mathrm{MgO}$ & $6.1 \mathrm{E}-05$ & $\mathrm{NiO}$ & $8.1 \mathrm{E}-05$ \\
\hline${ }^{226} \mathrm{RaO}$ & 5.5E-09 & $\mathrm{MoO}_{3}$ & $1.2 \mathrm{E}-04$ & $\mathrm{PbO}$ & 0.00011 \\
\hline${ }^{227} \mathrm{Ac}_{2} \mathrm{O}_{3}$ & 2.9E-14 & $\mathrm{Nd}_{2} \mathrm{O}_{3}$ & $4.2 \mathrm{E}-05$ & $\mathrm{P}_{2} \mathrm{O}_{5}$ & 0.0189 \\
\hline${ }^{228} \mathrm{RaO}$ & 1.6E-12 & $\mathrm{PdO}$ & 7.6E-08 & $\mathrm{SiO}_{2}$ & 0.0079 \\
\hline${ }^{229} \mathrm{ThO}_{2}$ & 2.3E-11 & $\mathrm{Pr}_{2} \mathrm{O}_{3}$ & $1.7 \mathrm{E}-07$ & $\mathrm{SO}_{3}$ & 0.0156 \\
\hline${ }^{231} \mathrm{~Pa}_{2} \mathrm{O}_{5}$ & $9.8 \mathrm{E}-11$ & $\mathrm{Rb}_{2} \mathrm{O}$ & $1.1 \mathrm{E}-06$ & $\mathrm{SrO}$ & $1.2 \mathrm{E}-05$ \\
\hline${ }^{232} \mathrm{ThO}_{2}$ & $1.0 \mathrm{E}-04$ & $\mathrm{Rh}_{2} \mathrm{O}_{3}$ & $2.4 \mathrm{E}-06$ & $\mathrm{ZrO}_{2}$ & $6.1 \mathrm{E}-05$ \\
\hline${ }^{232} \mathrm{UO}_{3}$ & $5.6 \mathrm{E}-13$ & $\mathrm{RuO}_{2}$ & 9.7E-06 & \multirow{26}{*}{\multicolumn{2}{|c|}{$\begin{array}{l}\text { Note that the oxide weight } \\
\text { fractions reported above } \\
\text { and immediately to the left } \\
\text { are for stable isotopes alone. }\end{array}$}} \\
\hline${ }^{233} \mathrm{UO}_{3}$ & 6.4E-09 & $\mathrm{Sb}_{2} \mathrm{O}_{3}$ & 4.3E-05 & & \\
\hline${ }^{234} \mathrm{UO}_{3}$ & $1.2 \mathrm{E}-08$ & $\mathrm{SeO}_{2}$ & 3.7E-04 & & \\
\hline${ }^{235} \mathrm{UO}_{3}$ & $1.7 \mathrm{E}-06$ & $\mathrm{Ta}_{2} \mathrm{O}_{5}$ & $2.0 \mathrm{E}-07$ & & \\
\hline${ }^{236} \mathrm{UO}_{3}$ & 4.7E-08 & $\mathrm{TeO}_{2}$ & $2.0 \mathrm{E}-07$ & & \\
\hline${ }^{237} \mathrm{NpO}_{2}$ & $8.5 \mathrm{E}-07$ & $\mathrm{ThO}_{2}$ & $1.2 \mathrm{E}-07$ & & \\
\hline${ }^{238} \mathrm{PuO}_{2}$ & $1.4 \mathrm{E}-11$ & $\mathrm{TiO}_{2}$ & 6.9E-06 & & \\
\hline${ }^{238} \mathrm{UO}_{3}$ & $2.6 \mathrm{E}-04$ & $\mathrm{Tl}_{2} \mathrm{O}$ & $7.0 \mathrm{E}-05$ & & \\
\hline${ }^{239} \mathrm{PuO}_{2}$ & $1.1 \mathrm{E}-07$ & $\mathrm{~V}_{2} \mathrm{O}_{5}$ & 2.9E-05 & & \\
\hline${ }^{240} \mathrm{PuO}_{2}$ & 5.4E-09 & $\mathrm{WO}_{3}$ & $3.2 \mathrm{E}-05$ & & \\
\hline${ }^{241} \mathrm{Am}_{2} \mathrm{O}_{3}$ & 2.2E-08 & $\mathrm{Y}_{2} \mathrm{O}_{3}$ & $4.2 \mathrm{E}-06$ & & \\
\hline${ }^{241} \mathrm{PuO}_{2}$ & 6.1E-11 & $\mathrm{ZnO}$ & $1.0 \mathrm{E}-05$ & & \\
\hline${ }^{242} \mathrm{Cm}_{2} \mathrm{O}_{3}$ & 3.7E-14 & & & & \\
\hline${ }^{242} \mathrm{PuO}_{2}$ & 2.5E-11 & & & & \\
\hline${ }^{243} \mathrm{Am}_{2} \mathrm{O}_{3}$ & $1.6 \mathrm{E}-11$ & & & & \\
\hline${ }^{243} \mathrm{Cm}_{2} \mathrm{O}_{3}$ & $2.1 \mathrm{E}-13$ & & & & \\
\hline${ }^{244} \mathrm{Cm}_{2} \mathrm{O}_{3}$ & $1.6 \mathrm{E}-12$ & & & & \\
\hline${ }^{59} \mathrm{NiO}$ & $1.4 \mathrm{E}-08$ & & & & \\
\hline${ }^{60} \mathrm{CoO}$ & $2.5 \mathrm{E}-12$ & & & & \\
\hline${ }^{63} \mathrm{NiO}$ & 1.7E-09 & & & & \\
\hline${ }^{79} \mathrm{SeO}_{2}$ & 3.3E-07 & & & & \\
\hline${ }^{90} \mathrm{SrO}$ & 5.5E-07 & & & & \\
\hline${ }^{90} \mathrm{Y}_{2} \mathrm{O}_{3}$ & 1.7E-09 & & & & \\
\hline${ }^{93} \mathrm{ZrO}_{2}$ & 7.3E-06 & & & & \\
\hline${ }^{93} \mathrm{Nb}_{2} \mathrm{O}_{5}$ & $7.9 \mathrm{E}-11$ & & & & \\
\hline${ }^{99} \mathrm{Tc}_{2} \mathrm{O}_{7}$ & 4.4E-05 & & & & \\
\hline
\end{tabular}


Table 5.2. Cluster 2 Waste Oxide Composition

\begin{tabular}{|c|c|c|c|c|c|}
\hline $\begin{array}{c}\text { Radioactive } \\
\text { oxides }\end{array}$ & $\begin{array}{l}\text { Wt. } \\
\text { frac. }\end{array}$ & $\begin{array}{c}\text { Supplemental } \\
\text { Species Oxides }\end{array}$ & $\begin{array}{l}\text { Wt. } \\
\text { frac. }\end{array}$ & $\begin{array}{c}\text { BBI Species } \\
\text { Oxides }\end{array}$ & Wt. frac. \\
\hline${ }^{106} \mathrm{RuO}_{2}$ & $4.1 \mathrm{E}-18$ & $\mathrm{Ag}_{2} \mathrm{O}$ & 7.7E-07 & $\mathrm{Al}_{2} \mathrm{O}_{3}$ & 0.128 \\
\hline${ }^{113} \mathrm{CdO}$ & $9.6 \mathrm{E}-11$ & $\mathrm{As}_{2} \mathrm{O}_{5}$ & $3.6 \mathrm{E}-06$ & $\mathrm{Bi}_{2} \mathrm{O}_{3}$ & 7.6E-05 \\
\hline${ }^{12} 5 \mathrm{Sb}_{2} \mathrm{O}_{3}$ & $9.8 \mathrm{E}-14$ & $\mathrm{~B}_{2} \mathrm{O}_{3}$ & $1.4 \mathrm{E}-05$ & $\mathrm{CaO}$ & 0.00046 \\
\hline${ }^{126} \mathrm{SnO}_{2}$ & 3.2E-08 & $\mathrm{BaO}$ & $1.0 \mathrm{E}-06$ & $\mathrm{Cl}$ & 0.009 \\
\hline${ }^{129} \mathrm{I}$ & 4.5E-06 & $\mathrm{BeO}$ & $3.9 \mathrm{E}-07$ & $\mathrm{Cr}_{2} \mathrm{O}_{3}$ & 0.0100 \\
\hline${ }^{134} \mathrm{Cs}_{2} \mathrm{O}$ & 8.8E-20 & $\mathrm{CdO}$ & $5.8 \mathrm{E}-06$ & $\mathrm{~F}$ & 0.0111 \\
\hline${ }^{137} \mathrm{Cs}_{2} \mathrm{O}$ & 2.7E-10 & $\mathrm{Ce}_{2} \mathrm{O}_{3}$ & $1.0 \mathrm{E}-03$ & $\mathrm{Fe}_{2} \mathrm{O}_{3}$ & 0.00046 \\
\hline${ }^{137} \mathrm{BaO}$ & 2.2E-13 & $\mathrm{Co}_{2} \mathrm{O}_{3}$ & $1.9 \mathrm{E}-06$ & $\mathrm{~K}_{2} \mathrm{O}$ & 0.004 \\
\hline${ }^{151} \mathrm{Sm}_{2} \mathrm{O}_{3}$ & 3.1E-08 & $\mathrm{Cs}_{2} \mathrm{O}$ & $1.3 \mathrm{E}-09$ & $\mathrm{La}_{2} \mathrm{O}_{3}$ & 7.7E-07 \\
\hline${ }^{152} \mathrm{Eu}_{2} \mathrm{O}_{3}$ & 8.4E-13 & $\mathrm{CuO}$ & 5.7E-07 & $\mathrm{MnO}$ & $2.0 \mathrm{E}-05$ \\
\hline${ }^{154} \mathrm{Eu}_{2} \mathrm{O}_{3}$ & 2.1E-11 & $\mathrm{Li}_{2} \mathrm{O}$ & 9.9E-07 & $\mathrm{Na}_{2} \mathrm{O}$ & 0.730 \\
\hline${ }^{15} 5 \mathrm{Eu}_{2} \mathrm{O}_{3}$ & 1.2E-12 & $\mathrm{MgO}$ & $5.1 \mathrm{E}-06$ & $\mathrm{NiO}$ & $6.4 \mathrm{E}-05$ \\
\hline${ }^{226} \mathrm{RaO}$ & 1.0E-08 & $\mathrm{MoO}_{3}$ & $8.4 \mathrm{E}-06$ & $\mathrm{PbO}$ & 0.00004 \\
\hline${ }^{227} \mathrm{Ac}_{2} \mathrm{O}_{3}$ & 2.0E-15 & $\mathrm{Nd}_{2} \mathrm{O}_{3}$ & $2.9 \mathrm{E}-06$ & $\mathrm{P}_{2} \mathrm{O}_{5}$ & 0.0644 \\
\hline${ }^{228} \mathrm{RaO}$ & $4.0 \mathrm{E}-13$ & $\mathrm{PdO}$ & $1.3 \mathrm{E}-08$ & $\mathrm{SiO}_{2}$ & 0.0088 \\
\hline${ }^{229} \mathrm{ThO}_{2}$ & 7.7E-12 & $\mathrm{Pr}_{2} \mathrm{O}_{3}$ & 3.7E-09 & $\mathrm{SO}_{3}$ & 0.0315 \\
\hline${ }^{231} \mathrm{~Pa}_{2} \mathrm{O}_{5}$ & 3.3E-11 & $\mathrm{Rb}_{2} \mathrm{O}$ & $2.3 \mathrm{E}-08$ & $\mathrm{SrO}$ & 3.5E-05 \\
\hline${ }^{232} \mathrm{ThO}_{2}$ & 3.3E-05 & $\mathrm{Rh}_{2} \mathrm{O}_{3}$ & $8.4 \mathrm{E}-08$ & $\mathrm{ZrO}_{2}$ & 0.00022 \\
\hline${ }^{232} \mathrm{UO}_{3}$ & 4.2E-13 & $\mathrm{RuO}_{2}$ & $2.1 \mathrm{E}-07$ & \multirow{26}{*}{\multicolumn{2}{|c|}{$\begin{array}{l}\text { Note that the oxide weight } \\
\text { fractions reported above } \\
\text { and immediately to the left } \\
\text { are for stable isotopes } \\
\text { alone. }\end{array}$}} \\
\hline${ }^{233} \mathrm{UO}_{3}$ & 5.0E-09 & $\mathrm{Sb}_{2} \mathrm{O}_{3}$ & $6.6 \mathrm{E}-06$ & & \\
\hline${ }^{234} \mathrm{UO}_{3}$ & 1.1E-08 & $\mathrm{SeO}_{2}$ & $1.0 \mathrm{E}-04$ & & \\
\hline${ }^{235} \mathrm{UO}_{3}$ & 1.3E-06 & $\mathrm{Ta}_{2} \mathrm{O}_{5}$ & $4.8 \mathrm{E}-08$ & & \\
\hline${ }^{236} \mathrm{UO}_{3}$ & 3.2E-08 & $\mathrm{TeO}_{2}$ & $5.4 \mathrm{E}-09$ & & \\
\hline${ }^{237} \mathrm{NpO}_{2}$ & 3.0E-07 & $\mathrm{ThO}_{2}$ & $1.4 \mathrm{E}-07$ & & \\
\hline${ }^{238} \mathrm{PuO}_{2}$ & $2.1 \mathrm{E}-10$ & $\mathrm{TiO}_{2}$ & $4.0 \mathrm{E}-07$ & & \\
\hline${ }^{238} \mathrm{UO}_{3}$ & $2.0 \mathrm{E}-04$ & $\mathrm{Tl}_{2} \mathrm{O}$ & $4.2 \mathrm{E}-06$ & & \\
\hline${ }^{239} \mathrm{PuO}_{2}$ & $6.0 \mathrm{E}-07$ & $\mathrm{~V}_{2} \mathrm{O}_{5}$ & $2.1 \mathrm{E}-06$ & & \\
\hline${ }^{240} \mathrm{PuO}_{2}$ & 2.8E-08 & $\mathrm{WO}_{3}$ & 5.5E-07 & & \\
\hline${ }^{241} \mathrm{Am}_{2} \mathrm{O}_{3}$ & 2.2E-08 & $\mathrm{Y}_{2} \mathrm{O}_{3}$ & $1.4 \mathrm{E}-07$ & & \\
\hline${ }^{241} \mathrm{PuO}_{2}$ & $1.5 \mathrm{E}-10$ & $\mathrm{ZnO}$ & $4.4 \mathrm{E}-06$ & & \\
\hline${ }^{242} \mathrm{Cm}_{2} \mathrm{O}_{3}$ & 3.0E-14 & & & & \\
\hline${ }^{242} \mathrm{PuO}_{2}$ & 8.8E-11 & & & & \\
\hline${ }^{243} \mathrm{Am}_{2} \mathrm{O}_{3}$ & 8.3E-12 & & & & \\
\hline${ }^{243} \mathrm{Cm}_{2} \mathrm{O}_{3}$ & $1.1 \mathrm{E}-13$ & & & & \\
\hline${ }^{244} \mathrm{Cm}_{2} \mathrm{O}_{3}$ & 8.9E-13 & & & & \\
\hline${ }^{59} \mathrm{NiO}$ & 8.1E-09 & & & & \\
\hline${ }^{60} \mathrm{CoO}$ & $2.8 \mathrm{E}-13$ & & & & \\
\hline${ }^{63} \mathrm{NiO}$ & $9.3 \mathrm{E}-10$ & & & & \\
\hline${ }^{79} \mathrm{SeO}_{2}$ & $1.7 \mathrm{E}-07$ & & & & \\
\hline${ }^{90} \mathrm{SrO}$ & 1.2E-06 & & & & \\
\hline${ }^{90} \mathrm{Y}_{2} \mathrm{O}_{3}$ & 1.7E-09 & & & & \\
\hline${ }^{93} \mathrm{ZrO}_{2}$ & 3.9E-06 & & & & \\
\hline${ }^{93} \mathrm{Nb}_{2} \mathrm{O}_{5}$ & 3.6E-11 & & & & \\
\hline${ }^{99} \mathrm{Tc}_{2} \mathrm{O}_{7}$ & 1.8E-05 & & & & \\
\hline
\end{tabular}


Table 5.3. Cluster 3 Waste Oxide Composition

\begin{tabular}{|c|c|c|c|c|c|}
\hline $\begin{array}{c}\text { Radioactive } \\
\text { oxides }\end{array}$ & Wt. frac. & $\begin{array}{l}\text { Supplemental } \\
\text { Species Oxides }\end{array}$ & $\begin{array}{l}\text { Wt. } \\
\text { frac. }\end{array}$ & $\begin{array}{l}\text { BBI Species } \\
\text { Oxides }\end{array}$ & Wt. frac. \\
\hline${ }^{106} \mathrm{RuO}_{2}$ & $8.8 \mathrm{E}-18$ & $\mathrm{Ag}_{2} \mathrm{O}$ & 5.7E-07 & $\mathrm{Al}_{2} \mathrm{O}_{3}$ & 0.158 \\
\hline${ }^{113} \mathrm{CdO}$ & 9.2E-11 & $\mathrm{As}_{2} \mathrm{O}_{5}$ & 4.4E-06 & $\mathrm{Bi}_{2} \mathrm{O}_{3}$ & $4.1 \mathrm{E}-05$ \\
\hline${ }^{12} 5 \mathrm{Sb}_{2} \mathrm{O}_{3}$ & $6.0 \mathrm{E}-14$ & $\mathrm{~B}_{2} \mathrm{O}_{3}$ & 1.3E-05 & $\mathrm{CaO}$ & 0.00026 \\
\hline${ }^{126} \mathrm{SnO}_{2}$ & 3.9E-08 & $\mathrm{BaO}$ & 1.0E-06 & $\mathrm{Cl}$ & 0.008 \\
\hline${ }^{129} \mathrm{I}$ & 4.7E-06 & $\mathrm{BeO}$ & $5.0 \mathrm{E}-07$ & $\mathrm{Cr}_{2} \mathrm{O}_{3}$ & 0.0089 \\
\hline${ }^{134} \mathrm{Cs}_{2} \mathrm{O}$ & $1.1 \mathrm{E}-19$ & $\mathrm{CdO}$ & 2.4E-06 & $\mathrm{F}$ & 0.0107 \\
\hline${ }^{137} \mathrm{Cs}_{2} \mathrm{O}$ & $2.4 \mathrm{E}-10$ & $\mathrm{Ce}_{2} \mathrm{O}_{3}$ & 8.9E-04 & $\mathrm{Fe}_{2} \mathrm{O}_{3}$ & 0.00043 \\
\hline${ }^{137} \mathrm{BaO}$ & $2.0 \mathrm{E}-13$ & $\mathrm{Co}_{2} \mathrm{O}_{3}$ & 1.3E-06 & $\mathrm{K}_{2} \mathrm{O}$ & 0.003 \\
\hline${ }^{151} \mathrm{Sm}_{2} \mathrm{O}_{3}$ & $4.4 \mathrm{E}-08$ & $\mathrm{Cs}_{2} \mathrm{O}$ & 1.3E-09 & $\mathrm{La}_{2} \mathrm{O}_{3}$ & $6.6 \mathrm{E}-07$ \\
\hline${ }^{152} \mathrm{Eu}_{2} \mathrm{O}_{3}$ & $9.3 \mathrm{E}-13$ & $\mathrm{CuO}$ & 5.5E-07 & $\mathrm{MnO}$ & $2.0 \mathrm{E}-05$ \\
\hline${ }^{154} \mathrm{Eu}_{2} \mathrm{O}_{3}$ & $2.6 \mathrm{E}-11$ & $\mathrm{Li}_{2} \mathrm{O}$ & 8.3E-07 & $\mathrm{Na}_{2} \mathrm{O}$ & 0.711 \\
\hline${ }^{15} 5 \mathrm{Eu}_{2} \mathrm{O}_{3}$ & $1.2 \mathrm{E}-12$ & $\mathrm{MgO}$ & 5.6E-06 & $\mathrm{NiO}$ & $8.5 \mathrm{E}-05$ \\
\hline${ }^{226} \mathrm{RaO}$ & 1.3E-09 & $\mathrm{MoO}_{3}$ & 9.6E-06 & $\mathrm{PbO}$ & 0.00004 \\
\hline${ }^{227} \mathrm{Ac}_{2} \mathrm{O}_{3}$ & $1.7 \mathrm{E}-15$ & $\mathrm{Nd}_{2} \mathrm{O}_{3}$ & 3.6E-06 & $\mathrm{P}_{2} \mathrm{O}_{5}$ & 0.0442 \\
\hline${ }^{228} \mathrm{RaO}$ & $1.1 \mathrm{E}-12$ & $\mathrm{PdO}$ & 9.0E-09 & $\mathrm{SiO}_{2}$ & 0.0071 \\
\hline${ }^{229} \mathrm{ThO}_{2}$ & $5.7 \mathrm{E}-12$ & $\mathrm{Pr}_{2} \mathrm{O}_{3}$ & 7.5E-09 & $\mathrm{SO}_{3}$ & 0.0459 \\
\hline${ }^{231} \mathrm{~Pa}_{2} \mathrm{O}_{5}$ & $3.1 \mathrm{E}-11$ & $\mathrm{Rb}_{2} \mathrm{O}$ & 3.3E-08 & $\mathrm{SrO}$ & $3.5 \mathrm{E}-05$ \\
\hline${ }^{232} \mathrm{ThO}_{2}$ & $2.9 \mathrm{E}-05$ & $\mathrm{Rh}_{2} \mathrm{O}_{3}$ & $6.5 \mathrm{E}-08$ & $\mathrm{ZrO}_{2}$ & $7.2 \mathrm{E}-05$ \\
\hline${ }^{232} \mathrm{UO}_{3}$ & $3.0 \mathrm{E}-13$ & $\mathrm{RuO}_{2}$ & 5.0E-07 & \multirow{26}{*}{\multicolumn{2}{|c|}{$\begin{array}{l}\text { Note that the oxide weight } \\
\text { fractions reported above } \\
\text { and immediately to the left } \\
\text { are for stable isotopes } \\
\text { alone. }\end{array}$}} \\
\hline${ }^{233} \mathrm{UO}_{3}$ & $3.6 \mathrm{E}-09$ & $\mathrm{Sb}_{2} \mathrm{O}_{3}$ & 4.3E-06 & & \\
\hline${ }^{234} \mathrm{UO}_{3}$ & 5.9E-09 & $\mathrm{SeO}_{2}$ & 7.1E-05 & & \\
\hline${ }^{235} \mathrm{UO}_{3}$ & $7.5 \mathrm{E}-07$ & $\mathrm{Ta}_{2} \mathrm{O}_{5}$ & 1.8E-09 & & \\
\hline${ }^{236} \mathrm{UO}_{3}$ & $1.5 \mathrm{E}-08$ & $\mathrm{TeO}_{2}$ & 1.5E-08 & & \\
\hline${ }^{237} \mathrm{NpO}_{2}$ & 3.3E-07 & $\mathrm{ThO}_{2}$ & 3.1E-08 & & \\
\hline${ }^{238} \mathrm{PuO}_{2}$ & $1.3 \mathrm{E}-10$ & $\mathrm{TiO}_{2}$ & 5.0E-07 & & \\
\hline${ }^{238} \mathrm{UO}_{3}$ & $1.2 \mathrm{E}-04$ & $\mathrm{Tl}_{2} \mathrm{O}$ & 7.2E-06 & & \\
\hline${ }^{239} \mathrm{PuO}_{2}$ & $4.0 \mathrm{E}-07$ & $\mathrm{~V}_{2} \mathrm{O}_{5}$ & 2.6E-06 & & \\
\hline${ }^{240} \mathrm{PuO}_{2}$ & $1.8 \mathrm{E}-08$ & $\mathrm{WO}_{3}$ & 7.9E-07 & & \\
\hline${ }^{241} \mathrm{Am}_{2} \mathrm{O}_{3}$ & 4.6E-09 & $\mathrm{Y}_{2} \mathrm{O}_{3}$ & 3.8E-07 & & \\
\hline${ }^{241} \mathrm{PuO}_{2}$ & $1.1 \mathrm{E}-10$ & $\mathrm{ZnO}$ & 1.7E-06 & & \\
\hline${ }^{242} \mathrm{Cm}_{2} \mathrm{O}_{3}$ & $2.2 \mathrm{E}-14$ & & & & \\
\hline${ }^{242} \mathrm{PuO}_{2}$ & 6.3E-11 & & & & \\
\hline${ }^{243} \mathrm{Am}_{2} \mathrm{O}_{3}$ & $2.3 \mathrm{E}-12$ & & & & \\
\hline${ }^{243} \mathrm{Cm}_{2} \mathrm{O}_{3}$ & 7.5E-14 & & & & \\
\hline${ }^{244} \mathrm{Cm}_{2} \mathrm{O}_{3}$ & $6.4 \mathrm{E}-13$ & & & & \\
\hline${ }^{59} \mathrm{NiO}$ & $1.5 \mathrm{E}-08$ & & & & \\
\hline${ }^{60} \mathrm{CoO}$ & $2.1 \mathrm{E}-13$ & & & & \\
\hline${ }^{63} \mathrm{NiO}$ & $1.8 \mathrm{E}-09$ & & & & \\
\hline${ }^{79} \mathrm{SeO}_{2}$ & $1.8 \mathrm{E}-07$ & & & & \\
\hline${ }^{90} \mathrm{SrO}$ & $2.6 \mathrm{E}-07$ & & & & \\
\hline${ }^{90} \mathrm{Y}_{2} \mathrm{O}_{3}$ & 1.3E-09 & & & & \\
\hline${ }^{93} \mathrm{ZrO}_{2}$ & $3.2 \mathrm{E}-06$ & & & & \\
\hline${ }^{93} \mathrm{Nb}_{2} \mathrm{O}_{5}$ & $4.1 \mathrm{E}-11$ & & & & \\
\hline${ }^{99} \mathrm{Tc}_{2} \mathrm{O}_{7}$ & $1.7 \mathrm{E}-05$ & & & & \\
\hline
\end{tabular}


Table 5.4. Cluster 4 Waste Oxide Composition

\begin{tabular}{|c|c|c|c|c|c|}
\hline $\begin{array}{c}\text { Radioactive } \\
\text { oxides }\end{array}$ & $\begin{array}{l}\text { Wt. } \\
\text { frac. }\end{array}$ & $\begin{array}{l}\text { Supplemental } \\
\text { Species Oxides }\end{array}$ & Wt. frac. & $\begin{array}{l}\text { BBI Species } \\
\text { Oxides }\end{array}$ & Wt. frac. \\
\hline${ }^{106} \mathrm{RuO}_{2}$ & $0.0 \mathrm{E}+00$ & $\mathrm{Ag}_{2} \mathrm{O}$ & $5.1 \mathrm{E}-07$ & $\mathrm{Al}_{2} \mathrm{O}_{3}$ & 0.193 \\
\hline${ }^{113} \mathrm{CdO}$ & 2.0E-11 & $\mathrm{As}_{2} \mathrm{O}_{5}$ & $2.0 \mathrm{E}-06$ & $\mathrm{Bi}_{2} \mathrm{O}_{3}$ & $1.8 \mathrm{E}-04$ \\
\hline${ }^{12} 5 \mathrm{Sb}_{2} \mathrm{O}_{3}$ & $6.5 \mathrm{E}-14$ & $\mathrm{~B}_{2} \mathrm{O}_{3}$ & 8.5E-06 & $\mathrm{CaO}$ & 0.00068 \\
\hline${ }^{126} \mathrm{SnO}_{2}$ & 1.3E-08 & $\mathrm{BaO}$ & 3.6E-07 & $\mathrm{Cl}$ & 0.004 \\
\hline${ }^{129} \mathrm{I}$ & 2.7E-06 & $\mathrm{BeO}$ & $1.7 \mathrm{E}-07$ & $\mathrm{Cr}_{2} \mathrm{O}_{3}$ & 0.0098 \\
\hline${ }^{134} \mathrm{Cs}_{2} \mathrm{O}$ & $0.0 \mathrm{E}+00$ & $\mathrm{CdO}$ & 2.3E-06 & $\mathrm{F}$ & 0.0111 \\
\hline${ }^{137} \mathrm{Cs}_{2} \mathrm{O}$ & $1.4 \mathrm{E}-10$ & $\mathrm{Ce}_{2} \mathrm{O}_{3}$ & $2.1 \mathrm{E}-03$ & $\mathrm{Fe}_{2} \mathrm{O}_{3}$ & 0.00080 \\
\hline${ }^{137} \mathrm{BaO}$ & $1.1 \mathrm{E}-13$ & $\mathrm{Co}_{2} \mathrm{O}_{3}$ & 2.0E-06 & $\mathrm{K}_{2} \mathrm{O}$ & 0.001 \\
\hline${ }^{151} \mathrm{Sm}_{2} \mathrm{O}_{3}$ & $1.7 \mathrm{E}-08$ & $\mathrm{Cs}_{2} \mathrm{O}$ & 7.7E-10 & $\mathrm{La}_{2} \mathrm{O}_{3}$ & $1.4 \mathrm{E}-07$ \\
\hline${ }^{152} \mathrm{Eu}_{2} \mathrm{O}_{3}$ & $9.5 \mathrm{E}-13$ & $\mathrm{CuO}$ & $2.3 \mathrm{E}-07$ & $\mathrm{MnO}$ & $1.1 \mathrm{E}-05$ \\
\hline${ }^{154} \mathrm{Eu}_{2} \mathrm{O}_{3}$ & $8.2 \mathrm{E}-12$ & $\mathrm{Li}_{2} \mathrm{O}$ & 3.3E-07 & $\mathrm{Na}_{2} \mathrm{O}$ & 0.652 \\
\hline${ }^{15} 5 \mathrm{Eu}_{2} \mathrm{O}_{3}$ & $5.7 \mathrm{E}-13$ & $\mathrm{MgO}$ & $3.4 \mathrm{E}-06$ & $\mathrm{NiO}$ & $3.2 \mathrm{E}-05$ \\
\hline${ }^{226} \mathrm{RaO}$ & $2.5 \mathrm{E}-08$ & $\mathrm{MoO}_{3}$ & $5.8 \mathrm{E}-06$ & $\mathrm{PbO}$ & 0.00005 \\
\hline${ }^{227} \mathrm{Ac}_{2} \mathrm{O}_{3}$ & $2.7 \mathrm{E}-15$ & $\mathrm{Nd}_{2} \mathrm{O}_{3}$ & $1.9 \mathrm{E}-06$ & $\mathrm{P}_{2} \mathrm{O}_{5}$ & 0.0893 \\
\hline${ }^{228} \mathrm{RaO}$ & $4.1 \mathrm{E}-13$ & $\mathrm{PdO}$ & 9.3E-08 & $\mathrm{SiO}_{2}$ & 0.0137 \\
\hline${ }^{229} \mathrm{ThO}_{2}$ & $1.6 \mathrm{E}-12$ & $\mathrm{Pr}_{2} \mathrm{O}_{3}$ & 7.6E-10 & $\mathrm{SO}_{3}$ & 0.0214 \\
\hline${ }^{231} \mathrm{~Pa}_{2} \mathrm{O}_{5}$ & $1.9 \mathrm{E}-11$ & $\mathrm{Rb}_{2} \mathrm{O}$ & $9.6 \mathrm{E}-08$ & $\mathrm{SrO}$ & $2.5 \mathrm{E}-05$ \\
\hline${ }^{232} \mathrm{ThO}_{2}$ & $8.1 \mathrm{E}-06$ & $\mathrm{Rh}_{2} \mathrm{O}_{3}$ & $1.7 \mathrm{E}-07$ & $\mathrm{ZrO}_{2}$ & $1.9 \mathrm{E}-05$ \\
\hline${ }^{232} \mathrm{UO}_{3}$ & $3.1 \mathrm{E}-13$ & $\mathrm{RuO}_{2}$ & $2.6 \mathrm{E}-07$ & \multirow{26}{*}{\multicolumn{2}{|c|}{$\begin{array}{l}\text { Note that the oxide weight } \\
\text { fractions reported above } \\
\text { and immediately to the left } \\
\text { are for stable isotopes } \\
\text { alone. }\end{array}$}} \\
\hline${ }^{233} \mathrm{UO}_{3}$ & 3.7E-09 & $\mathrm{Sb}_{2} \mathrm{O}_{3}$ & 4.7E-06 & & \\
\hline${ }^{234} \mathrm{UO}_{3}$ & $3.0 \mathrm{E}-08$ & $\mathrm{SeO}_{2}$ & $8.6 \mathrm{E}-05$ & & \\
\hline${ }^{235} \mathrm{UO}_{3}$ & $4.4 \mathrm{E}-06$ & $\mathrm{Ta}_{2} \mathrm{O}_{5}$ & $8.0 \mathrm{E}-10$ & & \\
\hline${ }^{236} \mathrm{UO}_{3}$ & $3.5 \mathrm{E}-08$ & $\mathrm{TeO}_{2}$ & $1.9 \mathrm{E}-09$ & & \\
\hline${ }^{237} \mathrm{NpO}_{2}$ & $1.5 \mathrm{E}-07$ & $\mathrm{ThO}_{2}$ & 1.9E-09 & & \\
\hline${ }^{238} \mathrm{PuO}_{2}$ & $3.0 \mathrm{E}-11$ & $\mathrm{TiO}_{2}$ & $2.8 \mathrm{E}-07$ & & \\
\hline${ }^{238} \mathrm{UO}_{3}$ & $5.6 \mathrm{E}-04$ & $\mathrm{Tl}_{2} \mathrm{O}$ & $4.5 \mathrm{E}-06$ & & \\
\hline${ }^{239} \mathrm{PuO}_{2}$ & $1.2 \mathrm{E}-07$ & $\mathrm{~V}_{2} \mathrm{O}_{5}$ & $1.1 \mathrm{E}-06$ & & \\
\hline${ }^{240} \mathrm{PuO}_{2}$ & 5.1E-09 & $\mathrm{WO}_{3}$ & $2.1 \mathrm{E}-07$ & & \\
\hline${ }^{241} \mathrm{Am}_{2} \mathrm{O}_{3}$ & 1.7E-09 & $\mathrm{Y}_{2} \mathrm{O}_{3}$ & $1.8 \mathrm{E}-08$ & & \\
\hline${ }^{241} \mathrm{PuO}_{2}$ & $2.7 \mathrm{E}-11$ & $\mathrm{ZnO}$ & 4.6E-06 & & \\
\hline${ }^{242} \mathrm{Cm}_{2} \mathrm{O}_{3}$ & $6.5 \mathrm{E}-15$ & & & & \\
\hline${ }^{242} \mathrm{PuO}_{2}$ & $1.7 \mathrm{E}-11$ & & & & \\
\hline${ }^{243} \mathrm{Am}_{2} \mathrm{O}_{3}$ & $5.5 \mathrm{E}-13$ & & & & \\
\hline${ }^{243} \mathrm{Cm}_{2} \mathrm{O}_{3}$ & 1.5E-14 & & & & \\
\hline${ }^{244} \mathrm{Cm}_{2} \mathrm{O}_{3}$ & $6.4 \mathrm{E}-14$ & & & & \\
\hline${ }^{59} \mathrm{NiO}$ & 3.6E-09 & & & & \\
\hline${ }^{60} \mathrm{CoO}$ & 5.3E-14 & & & & \\
\hline${ }^{63} \mathrm{NiO}$ & $4.1 \mathrm{E}-10$ & & & & \\
\hline${ }^{79} \mathrm{SeO}_{2}$ & $5.2 \mathrm{E}-08$ & & & & \\
\hline${ }^{90} \mathrm{SrO}$ & $5.9 \mathrm{E}-08$ & & & & \\
\hline${ }^{90} \mathrm{Y}_{2} \mathrm{O}_{3}$ & $1.5 \mathrm{E}-09$ & & & & \\
\hline${ }^{93} \mathrm{ZrO}_{2}$ & $9.2 \mathrm{E}-07$ & & & & \\
\hline${ }^{93} \mathrm{Nb}_{2} \mathrm{O}_{5}$ & $1.2 \mathrm{E}-11$ & & & & \\
\hline${ }^{99} \mathrm{Tc}_{2} \mathrm{O}_{7}$ & $1.4 \mathrm{E}-05$ & & & & \\
\hline
\end{tabular}


Table 5.5. Cluster 5 Waste Oxide Composition

\begin{tabular}{|c|c|c|c|c|c|}
\hline $\begin{array}{c}\text { Radioactive } \\
\text { oxides }\end{array}$ & Wt. frac. & $\begin{array}{l}\text { Supplemental } \\
\text { Species Oxides }\end{array}$ & Wt. frac. & $\begin{array}{c}\text { BBI Species } \\
\text { Oxides }\end{array}$ & Wt. frac. \\
\hline${ }^{106} \mathrm{RuO}_{2}$ & $0.0 \mathrm{E}+00$ & $\mathrm{Ag}_{2} \mathrm{O}$ & $3.3 \mathrm{E}-07$ & $\mathrm{Al}_{2} \mathrm{O}_{3}$ & 0.160 \\
\hline${ }^{113} \mathrm{CdO}$ & $8.1 \mathrm{E}-11$ & $\mathrm{As}_{2} \mathrm{O}_{5}$ & $1.2 \mathrm{E}-06$ & $\mathrm{Bi}_{2} \mathrm{O}_{3}$ & 8.7E-05 \\
\hline${ }^{12} 5 \mathrm{Sb}_{2} \mathrm{O}_{3}$ & $3.3 \mathrm{E}-14$ & $\mathrm{~B}_{2} \mathrm{O}_{3}$ & $4.9 \mathrm{E}-06$ & $\mathrm{CaO}$ & 0.00026 \\
\hline${ }^{126} \mathrm{SnO}_{2}$ & $1.2 \mathrm{E}-08$ & $\mathrm{BaO}$ & $4.1 \mathrm{E}-07$ & $\mathrm{Cl}$ & 0.009 \\
\hline${ }^{129} \mathrm{I}$ & 6.9E-06 & $\mathrm{BeO}$ & $1.1 \mathrm{E}-07$ & $\mathrm{Cr}_{2} \mathrm{O}_{3}$ & 0.0097 \\
\hline${ }^{134} \mathrm{Cs}_{2} \mathrm{O}$ & $0.0 \mathrm{E}+00$ & $\mathrm{CdO}$ & $1.2 \mathrm{E}-06$ & F & 0.0136 \\
\hline${ }^{137} \mathrm{Cs}_{2} \mathrm{O}$ & $1.8 \mathrm{E}-10$ & $\mathrm{Ce}_{2} \mathrm{O}_{3}$ & 3.5E-04 & $\mathrm{Fe}_{2} \mathrm{O}_{3}$ & 0.00044 \\
\hline${ }^{137} \mathrm{BaO}$ & $1.5 \mathrm{E}-13$ & $\mathrm{Co}_{2} \mathrm{O}_{3}$ & 3.7E-06 & $\mathrm{K}_{2} \mathrm{O}$ & 0.002 \\
\hline${ }^{151} \mathrm{Sm}_{2} \mathrm{O}_{3}$ & $1.5 \mathrm{E}-08$ & $\mathrm{Cs}_{2} \mathrm{O}$ & $1.0 \mathrm{E}-09$ & $\mathrm{La}_{2} \mathrm{O}_{3}$ & $1.7 \mathrm{E}-07$ \\
\hline${ }^{152} \mathrm{Eu}_{2} \mathrm{O}_{3}$ & $2.7 \mathrm{E}-13$ & $\mathrm{CuO}$ & $1.8 \mathrm{E}-07$ & $\mathrm{MnO}$ & $1.7 \mathrm{E}-05$ \\
\hline${ }^{154} \mathrm{Eu}_{2} \mathrm{O}_{3}$ & 1.3E-11 & $\mathrm{Li}_{2} \mathrm{O}$ & $2.2 \mathrm{E}-07$ & $\mathrm{Na}_{2} \mathrm{O}$ & 0.671 \\
\hline${ }^{15} 5 \mathrm{Eu}_{2} \mathrm{O}_{3}$ & $7.4 \mathrm{E}-13$ & $\mathrm{MgO}$ & $1.6 \mathrm{E}-06$ & $\mathrm{NiO}$ & 2.0E-05 \\
\hline${ }^{226} \mathrm{RaO}$ & $2.9 \mathrm{E}-08$ & $\mathrm{MoO}_{3}$ & $3.1 \mathrm{E}-06$ & $\mathrm{PbO}$ & 0.00003 \\
\hline${ }^{227} \mathrm{Ac}_{2} \mathrm{O}_{3}$ & $2.2 \mathrm{E}-15$ & $\mathrm{Nd}_{2} \mathrm{O}_{3}$ & $1.0 \mathrm{E}-06$ & $\mathrm{P}_{2} \mathrm{O}_{5}$ & 0.0526 \\
\hline${ }^{228} \mathrm{RaO}$ & $1.0 \mathrm{E}-12$ & $\mathrm{PdO}$ & 7.6E-11 & $\mathrm{SiO}_{2}$ & 0.0124 \\
\hline${ }^{229} \mathrm{ThO}_{2}$ & $1.2 \mathrm{E}-12$ & $\mathrm{Pr}_{2} \mathrm{O}_{3}$ & 4.7E-09 & $\mathrm{SO}_{3}$ & 0.0670 \\
\hline${ }^{231} \mathrm{~Pa}_{2} \mathrm{O}_{5}$ & $2.4 \mathrm{E}-11$ & $\mathrm{Rb}_{2} \mathrm{O}$ & $2.1 \mathrm{E}-08$ & $\mathrm{SrO}$ & 1.7E-05 \\
\hline${ }^{232} \mathrm{ThO}_{2}$ & 8.4E-06 & $\mathrm{Rh}_{2} \mathrm{O}_{3}$ & $3.5 \mathrm{E}-08$ & $\mathrm{ZrO}_{2}$ & 4.5E-05 \\
\hline${ }^{232} \mathrm{UO}_{3}$ & $2.3 \mathrm{E}-13$ & $\mathrm{RuO}_{2}$ & $1.6 \mathrm{E}-07$ & \multirow{26}{*}{\multicolumn{2}{|c|}{$\begin{array}{l}\text { Note that the oxide weight } \\
\text { fractions reported above } \\
\text { and immediately to the left } \\
\text { are for stable isotopes } \\
\text { alone. }\end{array}$}} \\
\hline${ }^{233} \mathrm{UO}_{3}$ & $2.7 \mathrm{E}-09$ & $\mathrm{Sb}_{2} \mathrm{O}_{3}$ & $3.0 \mathrm{E}-06$ & & \\
\hline${ }^{234} \mathrm{UO}_{3}$ & $8.5 \mathrm{E}-09$ & $\mathrm{SeO}_{2}$ & $1.2 \mathrm{E}-04$ & & \\
\hline${ }^{235} \mathrm{UO}_{3}$ & $1.2 \mathrm{E}-06$ & $\mathrm{Ta}_{2} \mathrm{O}_{5}$ & $3.6 \mathrm{E}-10$ & & \\
\hline${ }^{236} \mathrm{UO}_{3}$ & $1.3 \mathrm{E}-08$ & $\mathrm{TeO}_{2}$ & 5.7E-09 & & \\
\hline${ }^{237} \mathrm{NpO}_{2}$ & $2.2 \mathrm{E}-07$ & $\mathrm{ThO}_{2}$ & $6.0 \mathrm{E}-09$ & & \\
\hline${ }^{238} \mathrm{PuO}_{2}$ & $2.0 \mathrm{E}-11$ & $\mathrm{TiO}_{2}$ & $1.5 \mathrm{E}-07$ & & \\
\hline${ }^{238} \mathrm{UO}_{3}$ & $1.6 \mathrm{E}-04$ & $\mathrm{Tl}_{2} \mathrm{O}$ & $4.2 \mathrm{E}-06$ & & \\
\hline${ }^{239} \mathrm{PuO}_{2}$ & $2.0 \mathrm{E}-07$ & $\mathrm{~V}_{2} \mathrm{O}_{5}$ & 7.4E-07 & & \\
\hline${ }^{240} \mathrm{PuO}_{2}$ & 8.7E-09 & $\mathrm{WO}_{3}$ & $4.5 \mathrm{E}-07$ & & \\
\hline${ }^{241} \mathrm{Am}_{2} \mathrm{O}_{3}$ & 3.7E-09 & $\mathrm{Y}_{2} \mathrm{O}_{3}$ & $1.0 \mathrm{E}-07$ & & \\
\hline${ }^{241} \mathrm{PuO}_{2}$ & $5.2 \mathrm{E}-11$ & $\mathrm{ZnO}$ & 7.7E-07 & & \\
\hline${ }^{242} \mathrm{Cm}_{2} \mathrm{O}_{3}$ & $1.5 \mathrm{E}-14$ & & & & \\
\hline${ }^{242} \mathrm{PuO}_{2}$ & 2.8E-11 & & & & \\
\hline${ }^{243} \mathrm{Am}_{2} \mathrm{O}_{3}$ & $1.3 \mathrm{E}-12$ & & & & \\
\hline${ }^{243} \mathrm{Cm}_{2} \mathrm{O}_{3}$ & 5.3E-14 & & & & \\
\hline${ }^{244} \mathrm{Cm}_{2} \mathrm{O}_{3}$ & $2.7 \mathrm{E}-13$ & & & & \\
\hline${ }^{59} \mathrm{NiO}$ & $1.1 \mathrm{E}-08$ & & & & \\
\hline${ }^{60} \mathrm{CoO}$ & $1.7 \mathrm{E}-13$ & & & & \\
\hline${ }^{63} \mathrm{NiO}$ & $1.3 \mathrm{E}-09$ & & & & \\
\hline${ }^{79} \mathrm{SeO}_{2}$ & $8.5 \mathrm{E}-08$ & & & & \\
\hline${ }^{90} \mathrm{SrO}$ & $1.1 \mathrm{E}-07$ & & & & \\
\hline${ }^{90} \mathrm{Y}_{2} \mathrm{O}_{3}$ & 1.9E-09 & & & & \\
\hline${ }^{93} \mathrm{ZrO}_{2}$ & 2.7E-06 & & & & \\
\hline${ }^{93} \mathrm{Nb}_{2} \mathrm{O}_{5}$ & $1.7 \mathrm{E}-11$ & & & & \\
\hline${ }^{99} \mathrm{Tc}_{2} \mathrm{O}_{7}$ & $2.5 \mathrm{E}-05$ & & & & \\
\hline
\end{tabular}


Table 5.6. Cluster 6 Waste Oxide Composition

\begin{tabular}{|c|c|c|c|c|c|}
\hline $\begin{array}{c}\text { Radioactive } \\
\text { oxides }\end{array}$ & Wt. frac. & $\begin{array}{l}\text { Supplemental } \\
\text { Species Oxides }\end{array}$ & Wt. frac. & $\begin{array}{c}\text { BBI Species } \\
\text { Oxides }\end{array}$ & Wt. frac. \\
\hline${ }^{106} \mathrm{RuO}_{2}$ & $3.8 \mathrm{E}-18$ & $\mathrm{Ag}_{2} \mathrm{O}$ & $6.3 \mathrm{E}-07$ & $\mathrm{Al}_{2} \mathrm{O}_{3}$ & 0.121 \\
\hline${ }^{113} \mathrm{CdO}$ & $8.5 \mathrm{E}-11$ & $\mathrm{As}_{2} \mathrm{O}_{5}$ & $1.7 \mathrm{E}-06$ & $\mathrm{Bi}_{2} \mathrm{O}_{3}$ & $3.7 \mathrm{E}-05$ \\
\hline${ }^{12} 5 \mathrm{Sb}_{2} \mathrm{O}_{3}$ & $4.8 \mathrm{E}-14$ & $\mathrm{~B}_{2} \mathrm{O}_{3}$ & 7.5E-06 & $\mathrm{CaO}$ & 0.00054 \\
\hline${ }^{126} \mathrm{SnO}_{2}$ & 3.0E-08 & $\mathrm{BaO}$ & $1.5 \mathrm{E}-06$ & $\mathrm{Cl}$ & 0.007 \\
\hline${ }^{129} \mathrm{I}$ & $4.8 \mathrm{E}-06$ & $\mathrm{BeO}$ & $4.2 \mathrm{E}-07$ & $\mathrm{Cr}_{2} \mathrm{O}_{3}$ & 0.0063 \\
\hline${ }^{134} \mathrm{Cs}_{2} \mathrm{O}$ & 3.6E-20 & $\mathrm{CdO}$ & 2.5E-06 & $\mathrm{F}$ & 0.0162 \\
\hline${ }^{137} \mathrm{Cs}_{2} \mathrm{O}$ & $2.3 \mathrm{E}-10$ & $\mathrm{Ce}_{2} \mathrm{O}_{3}$ & 8.6E-04 & $\mathrm{Fe}_{2} \mathrm{O}_{3}$ & 0.00086 \\
\hline${ }^{137} \mathrm{BaO}$ & $1.9 \mathrm{E}-13$ & $\mathrm{Co}_{2} \mathrm{O}_{3}$ & $1.2 \mathrm{E}-06$ & $\mathrm{~K}_{2} \mathrm{O}$ & 0.005 \\
\hline${ }^{151} \mathrm{Sm}_{2} \mathrm{O}_{3}$ & $3.2 \mathrm{E}-08$ & $\mathrm{Cs}_{2} \mathrm{O}$ & $1.3 \mathrm{E}-09$ & $\mathrm{La}_{2} \mathrm{O}_{3}$ & $5.6 \mathrm{E}-07$ \\
\hline${ }^{152} \mathrm{Eu}_{2} \mathrm{O}_{3}$ & $8.6 \mathrm{E}-13$ & $\mathrm{CuO}$ & $4.4 \mathrm{E}-07$ & $\mathrm{MnO}$ & $1.7 \mathrm{E}-05$ \\
\hline${ }^{154} \mathrm{Eu}_{2} \mathrm{O}_{3}$ & $1.8 \mathrm{E}-11$ & $\mathrm{Li}_{2} \mathrm{O}$ & $6.8 \mathrm{E}-07$ & $\mathrm{Na}_{2} \mathrm{O}$ & 0.733 \\
\hline${ }^{15} 5 \mathrm{Eu}_{2} \mathrm{O}_{3}$ & $7.6 \mathrm{E}-13$ & $\mathrm{MgO}$ & $3.1 \mathrm{E}-06$ & $\mathrm{NiO}$ & $3.8 \mathrm{E}-04$ \\
\hline${ }^{226} \mathrm{RaO}$ & $1.2 \mathrm{E}-09$ & $\mathrm{MoO}_{3}$ & 7.3E-06 & $\mathrm{PbO}$ & 0.00005 \\
\hline${ }^{227} \mathrm{Ac}_{2} \mathrm{O}_{3}$ & $1.4 \mathrm{E}-15$ & $\mathrm{Nd}_{2} \mathrm{O}_{3}$ & $1.0 \mathrm{E}-06$ & $\mathrm{P}_{2} \mathrm{O}_{5}$ & 0.0410 \\
\hline${ }^{228} \mathrm{RaO}$ & $6.9 \mathrm{E}-13$ & PdO & $1.6 \mathrm{E}-08$ & $\mathrm{SiO}_{2}$ & 0.0092 \\
\hline${ }^{229} \mathrm{ThO}_{2}$ & $1.3 \mathrm{E}-11$ & $\mathrm{Pr}_{2} \mathrm{O}_{3}$ & $4.0 \mathrm{E}-09$ & $\mathrm{SO}_{3}$ & 0.0577 \\
\hline${ }^{231} \mathrm{~Pa}_{2} \mathrm{O}_{5}$ & $2.5 \mathrm{E}-11$ & $\mathrm{Rb}_{2} \mathrm{O}$ & $2.2 \mathrm{E}-08$ & $\mathrm{SrO}$ & $1.5 \mathrm{E}-04$ \\
\hline${ }^{232} \mathrm{ThO}_{2}$ & $6.8 \mathrm{E}-05$ & $\mathrm{Rh}_{2} \mathrm{O}_{3}$ & $4.6 \mathrm{E}-08$ & $\mathrm{ZrO}_{2}$ & $9.6 \mathrm{E}-05$ \\
\hline${ }^{232} \mathrm{UO}_{3}$ & $3.0 \mathrm{E}-13$ & $\mathrm{RuO}_{2}$ & $4.5 \mathrm{E}-07$ & \multirow{26}{*}{\multicolumn{2}{|c|}{$\begin{array}{l}\text { Note that the oxide weight } \\
\text { fractions reported above } \\
\text { and immediately to the left } \\
\text { are for stable isotopes } \\
\text { alone. }\end{array}$}} \\
\hline${ }^{233} \mathrm{UO}_{3}$ & $3.6 \mathrm{E}-09$ & $\mathrm{Sb}_{2} \mathrm{O}_{3}$ & $3.4 \mathrm{E}-06$ & & \\
\hline${ }^{234} \mathrm{UO}_{3}$ & $1.1 \mathrm{E}-08$ & $\mathrm{SeO}_{2}$ & $6.5 \mathrm{E}-05$ & & \\
\hline${ }^{235} \mathrm{UO}_{3}$ & $1.3 \mathrm{E}-06$ & $\mathrm{Ta}_{2} \mathrm{O}_{5}$ & $2.9 \mathrm{E}-09$ & & \\
\hline${ }^{236} \mathrm{UO}_{3}$ & $2.1 \mathrm{E}-08$ & $\mathrm{TeO}_{2}$ & $1.3 \mathrm{E}-08$ & & \\
\hline${ }^{237} \mathrm{NpO}_{2}$ & $3.5 \mathrm{E}-07$ & $\mathrm{ThO}_{2}$ & $2.5 \mathrm{E}-08$ & & \\
\hline${ }^{238} \mathrm{PuO}_{2}$ & $5.7 \mathrm{E}-11$ & $\mathrm{TiO}_{2}$ & $3.2 \mathrm{E}-07$ & & \\
\hline${ }^{238} \mathrm{UO}_{3}$ & $2.0 \mathrm{E}-04$ & $\mathrm{Tl}_{2} \mathrm{O}$ & $1.5 \mathrm{E}-06$ & & \\
\hline${ }^{239} \mathrm{PuO}_{2}$ & $2.4 \mathrm{E}-07$ & $\mathrm{~V}_{2} \mathrm{O}_{5}$ & 1.7E-06 & & \\
\hline${ }^{240} \mathrm{PuO}_{2}$ & $1.1 \mathrm{E}-08$ & $\mathrm{WO}_{3}$ & $6.6 \mathrm{E}-07$ & & \\
\hline${ }^{241} \mathrm{Am}_{2} \mathrm{O}_{3}$ & $3.0 \mathrm{E}-09$ & $\mathrm{Y}_{2} \mathrm{O}_{3}$ & $7.2 \mathrm{E}-08$ & & \\
\hline${ }^{241} \mathrm{PuO}_{2}$ & 6.6E-11 & $\mathrm{ZnO}$ & $1.0 \mathrm{E}-06$ & & \\
\hline${ }^{242} \mathrm{Cm}_{2} \mathrm{O}_{3}$ & $2.5 \mathrm{E}-14$ & & & & \\
\hline${ }^{242} \mathrm{PuO}_{2}$ & 3.7E-11 & & & & \\
\hline${ }^{243} \mathrm{Am}_{2} \mathrm{O}_{3}$ & $1.8 \mathrm{E}-12$ & & & & \\
\hline${ }^{243} \mathrm{Cm}_{2} \mathrm{O}_{3}$ & $4.1 \mathrm{E}-14$ & & & & \\
\hline${ }^{244} \mathrm{Cm}_{2} \mathrm{O}_{3}$ & $2.5 \mathrm{E}-13$ & & & & \\
\hline${ }^{59} \mathrm{NiO}$ & $1.8 \mathrm{E}-08$ & & & & \\
\hline${ }^{60} \mathrm{CoO}$ & $2.5 \mathrm{E}-13$ & & & & \\
\hline${ }^{63} \mathrm{NiO}$ & $2.1 \mathrm{E}-09$ & & & & \\
\hline${ }^{79} \mathrm{SeO}_{2}$ & $1.3 \mathrm{E}-07$ & & & & \\
\hline${ }^{90} \mathrm{SrO}$ & $1.9 \mathrm{E}-07$ & & & & \\
\hline${ }^{90} \mathrm{Y}_{2} \mathrm{O}_{3}$ & $7.9 \mathrm{E}-10$ & & & & \\
\hline${ }^{93} \mathrm{ZrO}_{2}$ & $2.4 \mathrm{E}-06$ & & & & \\
\hline${ }^{93} \mathrm{Nb}_{2} \mathrm{O}_{5}$ & $2.8 \mathrm{E}-11$ & & & & \\
\hline${ }^{99} \mathrm{Tc}_{2} \mathrm{O}_{7}$ & 1.3E-05 & & & & \\
\hline
\end{tabular}


Table 5.7. Cluster 7 Waste Oxide Composition

\begin{tabular}{|c|c|c|c|c|c|}
\hline $\begin{array}{c}\text { Radioactive } \\
\text { oxides }\end{array}$ & Wt. frac. & $\begin{array}{c}\text { Supplemental } \\
\text { Species Oxides }\end{array}$ & Wt. frac. & $\begin{array}{c}\text { BBI Species } \\
\text { Oxides }\end{array}$ & Wt. frac. \\
\hline${ }^{106} \mathrm{RuO}_{2}$ & 3.0E-17 & $\mathrm{Ag}_{2} \mathrm{O}$ & 3.3E-06 & $\mathrm{Al}_{2} \mathrm{O}_{3}$ & 0.122 \\
\hline${ }^{113} \mathrm{CdO}$ & $1.0 \mathrm{E}-10$ & $\mathrm{As}_{2} \mathrm{O}_{5}$ & 2.3E-05 & $\mathrm{Bi}_{2} \mathrm{O}_{3}$ & 7.1E-05 \\
\hline${ }^{12} 5 \mathrm{Sb}_{2} \mathrm{O}_{3}$ & $9.4 \mathrm{E}-13$ & $\mathrm{~B}_{2} \mathrm{O}_{3}$ & $8.8 \mathrm{E}-05$ & $\mathrm{CaO}$ & 0.00059 \\
\hline${ }^{126} \mathrm{SnO}_{2}$ & $1.1 \mathrm{E}-07$ & $\mathrm{BaO}$ & 7.5E-06 & $\mathrm{Cl}$ & 0.011 \\
\hline${ }^{129} \mathrm{I}$ & 5.7E-06 & $\mathrm{BeO}$ & $1.7 \mathrm{E}-06$ & $\mathrm{Cr}_{2} \mathrm{O}_{3}$ & 0.0078 \\
\hline${ }^{134} \mathrm{Cs}_{2} \mathrm{O}$ & 7.1E-18 & $\mathrm{CdO}$ & $3.0 \mathrm{E}-05$ & $\mathrm{~F}$ & 0.0073 \\
\hline${ }^{137} \mathrm{Cs}_{2} \mathrm{O}$ & 6.5E-10 & $\mathrm{Ce}_{2} \mathrm{O}_{3}$ & $1.0 \mathrm{E}-03$ & $\mathrm{Fe}_{2} \mathrm{O}_{3}$ & 0.00042 \\
\hline${ }^{137} \mathrm{BaO}$ & $5.5 \mathrm{E}-13$ & $\mathrm{Co}_{2} \mathrm{O}_{3}$ & $4.4 \mathrm{E}-06$ & $\mathrm{~K}_{2} \mathrm{O}$ & 0.006 \\
\hline${ }^{151} \mathrm{Sm}_{2} \mathrm{O}_{3}$ & $1.5 \mathrm{E}-07$ & $\mathrm{Cs}_{2} \mathrm{O}$ & 2.9E-09 & $\mathrm{La}_{2} \mathrm{O}_{3}$ & $8.2 \mathrm{E}-06$ \\
\hline${ }^{152} \mathrm{Eu}_{2} \mathrm{O}_{3}$ & $1.5 \mathrm{E}-11$ & $\mathrm{CuO}$ & $1.2 \mathrm{E}-05$ & $\mathrm{MnO}$ & $3.4 \mathrm{E}-05$ \\
\hline${ }^{154} \mathrm{Eu}_{2} \mathrm{O}_{3}$ & $2.2 \mathrm{E}-10$ & $\mathrm{Li}_{2} \mathrm{O}$ & 3.5E-06 & $\mathrm{Na}_{2} \mathrm{O}$ & 0.767 \\
\hline${ }^{15} 5 \mathrm{Eu}_{2} \mathrm{O}_{3}$ & 3.1E-11 & $\mathrm{MgO}$ & 2.9E-05 & $\mathrm{NiO}$ & $2.5 \mathrm{E}-04$ \\
\hline${ }^{226} \mathrm{RaO}$ & $8.0 \mathrm{E}-09$ & $\mathrm{MoO}_{3}$ & $6.2 \mathrm{E}-05$ & $\mathrm{PbO}$ & 0.00013 \\
\hline${ }^{227} \mathrm{Ac}_{2} \mathrm{O}_{3}$ & $1.4 \mathrm{E}-14$ & $\mathrm{Nd}_{2} \mathrm{O}_{3}$ & $1.8 \mathrm{E}-05$ & $\mathrm{P}_{2} \mathrm{O}_{5}$ & 0.0374 \\
\hline${ }^{228} \mathrm{RaO}$ & $1.7 \mathrm{E}-12$ & $\mathrm{PdO}$ & $5.2 \mathrm{E}-08$ & $\mathrm{SiO}_{2}$ & 0.0057 \\
\hline${ }^{229} \mathrm{ThO}_{2}$ & $1.8 \mathrm{E}-11$ & $\mathrm{Pr}_{2} \mathrm{O}_{3}$ & $3.4 \mathrm{E}-08$ & $\mathrm{SO}_{3}$ & 0.0318 \\
\hline${ }^{231} \mathrm{~Pa}_{2} \mathrm{O}_{5}$ & 6.8E-11 & $\mathrm{Rb}_{2} \mathrm{O}$ & $1.8 \mathrm{E}-07$ & $\mathrm{SrO}$ & $2.8 \mathrm{E}-05$ \\
\hline${ }^{232} \mathrm{ThO}_{2}$ & 5.4E-05 & $\mathrm{Rh}_{2} \mathrm{O}_{3}$ & 7.2E-07 & $\mathrm{ZrO}_{2}$ & 0.00012 \\
\hline${ }^{232} \mathrm{UO}_{3}$ & $4.9 \mathrm{E}-13$ & $\mathrm{RuO}_{2}$ & $1.3 \mathrm{E}-06$ & \multirow{26}{*}{\multicolumn{2}{|c|}{$\begin{array}{l}\text { Note that the oxide weight } \\
\text { fractions reported above } \\
\text { and immediately to the left } \\
\text { are for stable isotopes } \\
\text { alone. }\end{array}$}} \\
\hline${ }^{233} \mathrm{UO}_{3}$ & $6.2 \mathrm{E}-09$ & $\mathrm{Sb}_{2} \mathrm{O}_{3}$ & $1.7 \mathrm{E}-05$ & & \\
\hline${ }^{234} \mathrm{UO}_{3}$ & $1.1 \mathrm{E}-08$ & $\mathrm{SeO}_{2}$ & $2.7 \mathrm{E}-04$ & & \\
\hline${ }^{235} \mathrm{UO}_{3}$ & $1.6 \mathrm{E}-06$ & $\mathrm{Ta}_{2} \mathrm{O}_{5}$ & $3.5 \mathrm{E}-07$ & & \\
\hline${ }^{236} \mathrm{UO}_{3}$ & $4.1 \mathrm{E}-08$ & $\mathrm{TeO}_{2}$ & $4.1 \mathrm{E}-08$ & & \\
\hline${ }^{237} \mathrm{NpO}_{2}$ & $1.1 \mathrm{E}-06$ & $\mathrm{ThO}_{2}$ & $2.7 \mathrm{E}-07$ & & \\
\hline${ }^{238} \mathrm{PuO}_{2}$ & 4.2E-11 & $\mathrm{TiO}_{2}$ & $1.9 \mathrm{E}-06$ & & \\
\hline${ }^{238} \mathrm{UO}_{3}$ & $3.1 \mathrm{E}-04$ & $\mathrm{Tl}_{2} \mathrm{O}$ & $1.5 \mathrm{E}-05$ & & \\
\hline${ }^{239} \mathrm{PuO}_{2}$ & $2.8 \mathrm{E}-07$ & $\mathrm{~V}_{2} \mathrm{O}_{5}$ & $8.6 \mathrm{E}-06$ & & \\
\hline${ }^{240} \mathrm{PuO}_{2}$ & 1.3E-08 & $\mathrm{WO}_{3}$ & 4.1E-06 & & \\
\hline${ }^{241} \mathrm{Am}_{2} \mathrm{O}_{3}$ & $4.0 \mathrm{E}-08$ & $\mathrm{Y}_{2} \mathrm{O}_{3}$ & $1.7 \mathrm{E}-06$ & & \\
\hline${ }^{241} \mathrm{PuO}_{2}$ & $1.0 \mathrm{E}-10$ & $\mathrm{ZnO}$ & $4.0 \mathrm{E}-06$ & & \\
\hline${ }^{242} \mathrm{Cm}_{2} \mathrm{O}_{3}$ & $8.4 \mathrm{E}-14$ & & & & \\
\hline${ }^{242} \mathrm{PuO}_{2}$ & $5.0 \mathrm{E}-11$ & & & & \\
\hline${ }^{243} \mathrm{Am}_{2} \mathrm{O}_{3}$ & 2.9E-11 & & & & \\
\hline${ }^{243} \mathrm{Cm}_{2} \mathrm{O}_{3}$ & $3.5 \mathrm{E}-13$ & & & & \\
\hline${ }^{244} \mathrm{Cm}_{2} \mathrm{O}_{3}$ & $1.7 \mathrm{E}-12$ & & & & \\
\hline${ }^{59} \mathrm{NiO}$ & $1.1 \mathrm{E}-08$ & & & & \\
\hline${ }^{60} \mathrm{CoO}$ & $6.4 \mathrm{E}-12$ & & & & \\
\hline${ }^{63} \mathrm{NiO}$ & $1.3 \mathrm{E}-09$ & & & & \\
\hline${ }^{79} \mathrm{SeO}_{2}$ & $3.2 \mathrm{E}-07$ & & & & \\
\hline${ }^{90} \mathrm{SrO}$ & 8.5E-07 & & & & \\
\hline${ }^{90} \mathrm{Y}_{2} \mathrm{O}_{3}$ & $1.5 \mathrm{E}-09$ & & & & \\
\hline${ }^{93} \mathrm{ZrO}_{2}$ & 5.3E-06 & & & & \\
\hline${ }^{93} \mathrm{Nb}_{2} \mathrm{O}_{5}$ & 5.7E-11 & & & & \\
\hline${ }^{99} \mathrm{Tc}_{2} \mathrm{O}_{7}$ & $3.1 \mathrm{E}-05$ & & & & \\
\hline
\end{tabular}


Table 5.8. Cluster 8 Waste Oxide Composition

\begin{tabular}{|c|c|c|c|c|c|}
\hline $\begin{array}{c}\text { Radioactive } \\
\text { oxides }\end{array}$ & Wt. frac. & $\begin{array}{c}\text { Supplemental } \\
\text { Species Oxides }\end{array}$ & Wt. frac. & $\begin{array}{c}\text { BBI Species } \\
\text { Oxides }\end{array}$ & Wt. frac. \\
\hline${ }^{106} \mathrm{RuO}_{2}$ & $2.5 \mathrm{E}-17$ & $\mathrm{Ag}_{2} \mathrm{O}$ & 5.2E-06 & $\mathrm{Al}_{2} \mathrm{O}_{3}$ & 0.096 \\
\hline${ }^{113} \mathrm{CdO}$ & $1.7 \mathrm{E}-10$ & $\mathrm{As}_{2} \mathrm{O}_{5}$ & 1.6E-05 & $\mathrm{Bi}_{2} \mathrm{O}_{3}$ & $5.2 \mathrm{E}-05$ \\
\hline${ }^{12} 5 \mathrm{Sb}_{2} \mathrm{O}_{3}$ & 7.4E-11 & $\mathrm{B}_{2} \mathrm{O}_{3}$ & 7.1E-05 & $\mathrm{CaO}$ & 0.00037 \\
\hline${ }^{126} \mathrm{SnO}_{2}$ & $1.5 \mathrm{E}-07$ & $\mathrm{BaO}$ & 4.4E-06 & $\mathrm{Cl}$ & 0.010 \\
\hline${ }^{129} \mathrm{I}$ & $6.0 \mathrm{E}-06$ & $\mathrm{BeO}$ & $1.4 \mathrm{E}-06$ & $\mathrm{Cr}_{2} \mathrm{O}_{3}$ & 0.0069 \\
\hline${ }^{134} \mathrm{Cs}_{2} \mathrm{O}$ & 4.0E-15 & $\mathrm{CdO}$ & 9.7E-06 & $\mathrm{F}$ & 0.0071 \\
\hline${ }^{137} \mathrm{Cs}_{2} \mathrm{O}$ & $1.8 \mathrm{E}-09$ & $\mathrm{Ce}_{2} \mathrm{O}_{3}$ & 7.5E-04 & $\mathrm{Fe}_{2} \mathrm{O}_{3}$ & 0.00041 \\
\hline${ }^{137} \mathrm{BaO}$ & $1.7 \mathrm{E}-12$ & $\mathrm{Co}_{2} \mathrm{O}_{3}$ & 2.7E-06 & $\mathrm{K}_{2} \mathrm{O}$ & 0.009 \\
\hline${ }^{151} \mathrm{Sm}_{2} \mathrm{O}_{3}$ & $2.5 \mathrm{E}-07$ & $\mathrm{Cs}_{2} \mathrm{O}$ & 5.4E-09 & $\mathrm{La}_{2} \mathrm{O}_{3}$ & $2.3 \mathrm{E}-06$ \\
\hline${ }^{152} \mathrm{Eu}_{2} \mathrm{O}_{3}$ & $7.6 \mathrm{E}-11$ & $\mathrm{CuO}$ & $2.8 \mathrm{E}-06$ & $\mathrm{MnO}$ & $2.3 \mathrm{E}-05$ \\
\hline${ }^{154} \mathrm{Eu}_{2} \mathrm{O}_{3}$ & $2.0 \mathrm{E}-10$ & $\mathrm{Li}_{2} \mathrm{O}$ & 3.1E-06 & $\mathrm{Na}_{2} \mathrm{O}$ & 0.779 \\
\hline${ }^{15} 5 \mathrm{Eu}_{2} \mathrm{O}_{3}$ & $8.2 \mathrm{E}-11$ & $\mathrm{MgO}$ & 2.7E-05 & $\mathrm{NiO}$ & $1.0 \mathrm{E}-04$ \\
\hline${ }^{226} \mathrm{RaO}$ & 6.1E-09 & $\mathrm{MoO}_{3}$ & 1.1E-04 & $\mathrm{PbO}$ & 0.00007 \\
\hline${ }^{227} \mathrm{Ac}_{2} \mathrm{O}_{3}$ & $1.7 \mathrm{E}-14$ & $\mathrm{Nd}_{2} \mathrm{O}_{3}$ & $1.7 \mathrm{E}-05$ & $\mathrm{P}_{2} \mathrm{O}_{5}$ & 0.0273 \\
\hline${ }^{228} \mathrm{RaO}$ & $2.9 \mathrm{E}-13$ & $\mathrm{PdO}$ & 4.8E-06 & $\mathrm{SiO}_{2}$ & 0.0067 \\
\hline${ }^{229} \mathrm{ThO}_{2}$ & $1.3 \mathrm{E}-11$ & $\mathrm{Pr}_{2} \mathrm{O}_{3}$ & 4.9E-08 & $\mathrm{SO}_{3}$ & 0.0548 \\
\hline${ }^{231} \mathrm{~Pa}_{2} \mathrm{O}_{5}$ & $8.8 \mathrm{E}-11$ & $\mathrm{Rb}_{2} \mathrm{O}$ & 3.5E-06 & $\mathrm{SrO}$ & $3.6 \mathrm{E}-05$ \\
\hline${ }^{232} \mathrm{ThO}_{2}$ & $5.2 \mathrm{E}-05$ & $\mathrm{Rh}_{2} \mathrm{O}_{3}$ & 4.5E-06 & $\mathrm{ZrO}_{2}$ & $9.4 \mathrm{E}-05$ \\
\hline${ }^{232} \mathrm{UO}_{3}$ & $4.1 \mathrm{E}-13$ & $\mathrm{RuO}_{2}$ & 7.8E-06 & \multirow{26}{*}{\multicolumn{2}{|c|}{$\begin{array}{l}\text { Note that the oxide weight } \\
\text { fractions reported above } \\
\text { and immediately to the left } \\
\text { are for stable isotopes } \\
\text { alone. }\end{array}$}} \\
\hline${ }^{233} \mathrm{UO}_{3}$ & 5.2E-09 & $\mathrm{Sb}_{2} \mathrm{O}_{3}$ & 1.3E-05 & & \\
\hline${ }^{234} \mathrm{UO}_{3}$ & $1.7 \mathrm{E}-08$ & $\mathrm{SeO}_{2}$ & $2.0 \mathrm{E}-04$ & & \\
\hline${ }^{235} \mathrm{UO}_{3}$ & $1.8 \mathrm{E}-06$ & $\mathrm{Ta}_{2} \mathrm{O}_{5}$ & 4.4E-08 & & \\
\hline${ }^{236} \mathrm{UO}_{3}$ & 5.7E-08 & $\mathrm{TeO}_{2}$ & $1.7 \mathrm{E}-07$ & & \\
\hline${ }^{237} \mathrm{NpO}_{2}$ & $7.5 \mathrm{E}-07$ & $\mathrm{ThO}_{2}$ & 7.5E-08 & & \\
\hline${ }^{238} \mathrm{PuO}_{2}$ & $6.2 \mathrm{E}-11$ & $\mathrm{TiO}_{2}$ & 2.6E-06 & & \\
\hline${ }^{238} \mathrm{UO}_{3}$ & $3.2 \mathrm{E}-04$ & $\mathrm{Tl}_{2} \mathrm{O}$ & 6.7E-06 & & \\
\hline${ }^{239} \mathrm{PuO}_{2}$ & $3.8 \mathrm{E}-07$ & $\mathrm{~V}_{2} \mathrm{O}_{5}$ & 7.2E-06 & & \\
\hline${ }^{240} \mathrm{PuO}_{2}$ & $2.1 \mathrm{E}-08$ & $\mathrm{WO}_{3}$ & 1.6E-05 & & \\
\hline${ }^{241} \mathrm{Am}_{2} \mathrm{O}_{3}$ & $6.1 \mathrm{E}-08$ & $\mathrm{Y}_{2} \mathrm{O}_{3}$ & $8.0 \mathrm{E}-07$ & & \\
\hline${ }^{241} \mathrm{PuO}_{2}$ & $4.2 \mathrm{E}-10$ & $\mathrm{ZnO}$ & 5.6E-06 & & \\
\hline${ }^{242} \mathrm{Cm}_{2} \mathrm{O}_{3}$ & 5.7E-14 & & & & \\
\hline${ }^{242} \mathrm{PuO}_{2}$ & $1.4 \mathrm{E}-10$ & & & & \\
\hline${ }^{243} \mathrm{Am}_{2} \mathrm{O}_{3}$ & $2.4 \mathrm{E}-10$ & & & & \\
\hline${ }^{243} \mathrm{Cm}_{2} \mathrm{O}_{3}$ & $2.9 \mathrm{E}-13$ & & & & \\
\hline${ }^{244} \mathrm{Cm}_{2} \mathrm{O}_{3}$ & $2.5 \mathrm{E}-12$ & & & & \\
\hline${ }^{59} \mathrm{NiO}$ & 1.3E-08 & & & & \\
\hline${ }^{60} \mathrm{CoO}$ & $5.5 \mathrm{E}-12$ & & & & \\
\hline${ }^{63} \mathrm{NiO}$ & $1.6 \mathrm{E}-09$ & & & & \\
\hline${ }^{79} \mathrm{SeO}_{2}$ & $3.0 \mathrm{E}-07$ & & & & \\
\hline${ }^{90} \mathrm{SrO}$ & $1.3 \mathrm{E}-06$ & & & & \\
\hline${ }^{90} \mathrm{Y}_{2} \mathrm{O}_{3}$ & $2.5 \mathrm{E}-09$ & & & & \\
\hline${ }^{93} \mathrm{ZrO}_{2}$ & 7.7E-06 & & & & \\
\hline${ }^{93} \mathrm{Nb}_{2} \mathrm{O}_{5}$ & $9.6 \mathrm{E}-11$ & & & & \\
\hline${ }^{99} \mathrm{Tc}_{2} \mathrm{O}_{7}$ & $4.2 \mathrm{E}-05$ & & & & \\
\hline
\end{tabular}


Table 5.9. Cluster 9 Waste Oxide Composition

\begin{tabular}{|c|c|c|c|c|c|}
\hline $\begin{array}{c}\text { Radioactive } \\
\text { oxides }\end{array}$ & Wt. frac. & $\begin{array}{c}\text { Supplemental } \\
\text { Species Oxides }\end{array}$ & Wt. frac. & $\begin{array}{c}\text { BBI Species } \\
\text { Oxides } \\
\end{array}$ & Wt. frac. \\
\hline${ }^{106} \mathrm{RuO}_{2}$ & $2.5 \mathrm{E}-18$ & $\mathrm{Ag}_{2} \mathrm{O}$ & $3.1 \mathrm{E}-06$ & $\mathrm{Al}_{2} \mathrm{O}_{3}$ & 0.102 \\
\hline${ }^{113} \mathrm{CdO}$ & $1.2 \mathrm{E}-09$ & $\mathrm{As}_{2} \mathrm{O}_{5}$ & 5.9E-05 & $\mathrm{Bi}_{2} \mathrm{O}_{3}$ & 3.5E-06 \\
\hline${ }^{12} 5 \mathrm{Sb}_{2} \mathrm{O}_{3}$ & $5.0 \mathrm{E}-10$ & $\mathrm{~B}_{2} \mathrm{O}_{3}$ & 2.4E-04 & $\mathrm{CaO}$ & 0.00032 \\
\hline${ }^{126} \mathrm{SnO}_{2}$ & $1.0 \mathrm{E}-06$ & $\mathrm{BaO}$ & 7.9E-07 & $\mathrm{Cl}$ & 0.002 \\
\hline${ }^{129} \mathrm{I}$ & $4.8 \mathrm{E}-06$ & $\mathrm{BeO}$ & 3.4E-06 & $\mathrm{Cr}_{2} \mathrm{O}_{3}$ & 0.0044 \\
\hline${ }^{134} \mathrm{Cs}_{2} \mathrm{O}$ & $1.6 \mathrm{E}-14$ & $\mathrm{CdO}$ & 4.3E-06 & $\mathrm{F}$ & 0.0105 \\
\hline${ }^{137} \mathrm{Cs}_{2} \mathrm{O}$ & 2.2E-09 & $\mathrm{Ce}_{2} \mathrm{O}_{3}$ & 7.5E-04 & $\mathrm{Fe}_{2} \mathrm{O}_{3}$ & 0.00282 \\
\hline${ }^{137} \mathrm{BaO}$ & $1.0 \mathrm{E}-11$ & $\mathrm{Co}_{2} \mathrm{O}_{3}$ & 6.3E-07 & $\mathrm{K}_{2} \mathrm{O}$ & 0.023 \\
\hline${ }^{151} \mathrm{Sm}_{2} \mathrm{O}_{3}$ & $2.6 \mathrm{E}-06$ & $\mathrm{Cs}_{2} \mathrm{O}$ & 5.6E-09 & $\mathrm{La}_{2} \mathrm{O}_{3}$ & 4.1E-07 \\
\hline${ }^{152} \mathrm{Eu}_{2} \mathrm{O}_{3}$ & $1.2 \mathrm{E}-09$ & $\mathrm{CuO}$ & 3.3E-06 & $\mathrm{MnO}$ & $8.2 \mathrm{E}-07$ \\
\hline${ }^{154} \mathrm{Eu}_{2} \mathrm{O}_{3}$ & $4.8 \mathrm{E}-10$ & $\mathrm{Li}_{2} \mathrm{O}$ & 7.7E-06 & $\mathrm{Na}_{2} \mathrm{O}$ & 0.758 \\
\hline${ }^{15} 5 \mathrm{Eu}_{2} \mathrm{O}_{3}$ & $1.8 \mathrm{E}-10$ & $\mathrm{MgO}$ & $2.0 \mathrm{E}-05$ & $\mathrm{NiO}$ & $1.7 \mathrm{E}-05$ \\
\hline${ }^{226} \mathrm{RaO}$ & $1.6 \mathrm{E}-10$ & $\mathrm{MoO}_{3}$ & 5.3E-04 & $\mathrm{PbO}$ & 0.00003 \\
\hline${ }^{227} \mathrm{Ac}_{2} \mathrm{O}_{3}$ & $1.4 \mathrm{E}-13$ & $\mathrm{Nd}_{2} \mathrm{O}_{3}$ & $1.3 \mathrm{E}-05$ & $\mathrm{P}_{2} \mathrm{O}_{5}$ & 0.0068 \\
\hline${ }^{228} \mathrm{RaO}$ & $2.4 \mathrm{E}-13$ & PdO & 8.7E-05 & $\mathrm{SiO}_{2}$ & 0.0030 \\
\hline${ }^{229} \mathrm{ThO}_{2}$ & $9.3 \mathrm{E}-13$ & $\mathrm{Pr}_{2} \mathrm{O}_{3}$ & 2.7E-07 & $\mathrm{SO}_{3}$ & 0.0846 \\
\hline${ }^{231} \mathrm{~Pa}_{2} \mathrm{O}_{5}$ & $4.5 \mathrm{E}-10$ & $\mathrm{Rb}_{2} \mathrm{O}$ & 3.9E-05 & $\mathrm{SrO}$ & 2.9E-06 \\
\hline${ }^{232} \mathrm{ThO}_{2}$ & $9.5 \mathrm{E}-06$ & $\mathrm{Rh}_{2} \mathrm{O}_{3}$ & 1.1E-05 & $\mathrm{ZrO}_{2}$ & 2.6E-06 \\
\hline${ }^{232} \mathrm{UO}_{3}$ & $8.8 \mathrm{E}-14$ & $\mathrm{RuO}_{2}$ & $4.9 \mathrm{E}-06$ & \multirow{26}{*}{\multicolumn{2}{|c|}{$\begin{array}{l}\text { Note that the oxide weight } \\
\text { fractions reported above } \\
\text { and immediately to the left } \\
\text { are for stable isotopes } \\
\text { alone. }\end{array}$}} \\
\hline${ }^{233} \mathrm{UO}_{3}$ & $1.1 \mathrm{E}-09$ & $\mathrm{Sb}_{2} \mathrm{O}_{3}$ & 7.9E-07 & & \\
\hline${ }^{234} \mathrm{UO}_{3}$ & $4.7 \mathrm{E}-09$ & $\mathrm{SeO}_{2}$ & $6.5 \mathrm{E}-05$ & & \\
\hline${ }^{235} \mathrm{UO}_{3}$ & $5.2 \mathrm{E}-07$ & $\mathrm{Ta}_{2} \mathrm{O}_{5}$ & $2.8 \mathrm{E}-07$ & & \\
\hline${ }^{236} \mathrm{UO}_{3}$ & $2.3 \mathrm{E}-08$ & $\mathrm{TeO}_{2}$ & 3.7E-07 & & \\
\hline${ }^{237} \mathrm{NpO}_{2}$ & 3.9E-07 & $\mathrm{ThO}_{2}$ & 2.4E-07 & & \\
\hline${ }^{238} \mathrm{PuO}_{2}$ & $2.0 \mathrm{E}-10$ & $\mathrm{TiO}_{2}$ & 4.4E-06 & & \\
\hline${ }^{238} \mathrm{UO}_{3}$ & 7.3E-05 & $\mathrm{Tl}_{2} \mathrm{O}$ & 2.3E-06 & & \\
\hline${ }^{239} \mathrm{PuO}_{2}$ & 5.3E-07 & $\mathrm{V}_{2} \mathrm{O}_{5}$ & $1.1 \mathrm{E}-05$ & & \\
\hline${ }^{240} \mathrm{PuO}_{2}$ & 4.4E-08 & $\mathrm{WO}_{3}$ & 2.8E-04 & & \\
\hline${ }^{241} \mathrm{Am}_{2} \mathrm{O}_{3}$ & $1.1 \mathrm{E}-07$ & $\mathrm{Y}_{2} \mathrm{O}_{3}$ & 6.5E-06 & & \\
\hline${ }^{241} \mathrm{PuO}_{2}$ & $1.8 \mathrm{E}-09$ & $\mathrm{ZnO}$ & 6.5E-06 & & \\
\hline${ }^{242} \mathrm{Cm}_{2} \mathrm{O}_{3}$ & $4.3 \mathrm{E}-13$ & & & & \\
\hline${ }^{242} \mathrm{PuO}_{2}$ & $4.0 \mathrm{E}-10$ & & & & \\
\hline${ }^{243} \mathrm{Am}_{2} \mathrm{O}_{3}$ & $4.1 \mathrm{E}-10$ & & & & \\
\hline${ }^{243} \mathrm{Cm}_{2} \mathrm{O}_{3}$ & 2.7E-12 & & & & \\
\hline${ }^{244} \mathrm{Cm}_{2} \mathrm{O}_{3}$ & $3.6 \mathrm{E}-11$ & & & & \\
\hline${ }^{59} \mathrm{NiO}$ & 5.8E-09 & & & & \\
\hline${ }^{60} \mathrm{CoO}$ & 3.9E-11 & & & & \\
\hline${ }^{63} \mathrm{NiO}$ & 7.5E-10 & & & & \\
\hline${ }^{79} \mathrm{SeO}_{2}$ & $8.4 \mathrm{E}-07$ & & & & \\
\hline${ }^{90} \mathrm{SrO}$ & $7.8 \mathrm{E}-07$ & & & & \\
\hline${ }^{90} \mathrm{Y}_{2} \mathrm{O}_{3}$ & $1.1 \mathrm{E}-08$ & & & & \\
\hline${ }^{93} \mathrm{ZrO}_{2}$ & $4.8 \mathrm{E}-05$ & & & & \\
\hline${ }^{93} \mathrm{Nb}_{2} \mathrm{O}_{5}$ & $6.8 \mathrm{E}-10$ & & & & \\
\hline${ }^{99} \mathrm{Tc}_{2} \mathrm{O}_{7}$ & $1.8 \mathrm{E}-04$ & & & & \\
\hline
\end{tabular}


Table 5.10. Cluster 10 Waste Oxide Composition

\begin{tabular}{|c|c|c|c|c|c|}
\hline $\begin{array}{c}\text { Radioactive } \\
\text { oxides }\end{array}$ & Wt. frac. & $\begin{array}{l}\text { Supplemental } \\
\text { Species Oxides }\end{array}$ & Wt. frac. & $\begin{array}{c}\text { BBI Species } \\
\text { Oxides }\end{array}$ & Wt. frac. \\
\hline${ }^{106} \mathrm{RuO}_{2}$ & $4.0 \mathrm{E}-18$ & $\mathrm{Ag}_{2} \mathrm{O}$ & $3.0 \mathrm{E}-06$ & $\mathrm{Al}_{2} \mathrm{O}_{3}$ & 0.244 \\
\hline${ }^{113} \mathrm{CdO}$ & $4.1 \mathrm{E}-11$ & $\mathrm{As}_{2} \mathrm{O}_{5}$ & 7.1E-06 & $\mathrm{Bi}_{2} \mathrm{O}_{3}$ & $2.8 \mathrm{E}-05$ \\
\hline${ }^{12} 5 \mathrm{Sb}_{2} \mathrm{O}_{3}$ & $2.8 \mathrm{E}-13$ & $\mathrm{~B}_{2} \mathrm{O}_{3}$ & 4.6E-05 & $\mathrm{CaO}$ & 0.00023 \\
\hline${ }^{126} \mathrm{SnO}_{2}$ & $1.4 \mathrm{E}-07$ & $\mathrm{BaO}$ & $1.5 \mathrm{E}-06$ & $\mathrm{Cl}$ & 0.009 \\
\hline${ }^{129} \mathrm{I}$ & $4.1 \mathrm{E}-06$ & $\mathrm{BeO}$ & 8.7E-07 & $\mathrm{Cr}_{2} \mathrm{O}_{3}$ & 0.0121 \\
\hline${ }^{134} \mathrm{Cs}_{2} \mathrm{O}$ & 2.4E-18 & $\mathrm{CdO}$ & $5.0 \mathrm{E}-06$ & $\mathrm{~F}$ & 0.0037 \\
\hline${ }^{137} \mathrm{Cs}_{2} \mathrm{O}$ & $5.1 \mathrm{E}-10$ & $\mathrm{Ce}_{2} \mathrm{O}_{3}$ & $1.4 \mathrm{E}-03$ & $\mathrm{Fe}_{2} \mathrm{O}_{3}$ & 0.00021 \\
\hline${ }^{137} \mathrm{BaO}$ & $4.2 \mathrm{E}-13$ & $\mathrm{Co}_{2} \mathrm{O}_{3}$ & 1.6E-06 & $\mathrm{K}_{2} \mathrm{O}$ & 0.009 \\
\hline${ }^{151} \mathrm{Sm}_{2} \mathrm{O}_{3}$ & $6.0 \mathrm{E}-08$ & $\mathrm{Cs}_{2} \mathrm{O}$ & $1.7 \mathrm{E}-09$ & $\mathrm{La}_{2} \mathrm{O}_{3}$ & $7.9 \mathrm{E}-07$ \\
\hline${ }^{152} \mathrm{Eu}_{2} \mathrm{O}_{3}$ & $2.3 \mathrm{E}-12$ & $\mathrm{CuO}$ & $1.1 \mathrm{E}-06$ & $\mathrm{MnO}$ & 8.3E-06 \\
\hline${ }^{154} \mathrm{Eu}_{2} \mathrm{O}_{3}$ & 2.3E-11 & $\mathrm{Li}_{2} \mathrm{O}$ & 7.1E-06 & $\mathrm{Na}_{2} \mathrm{O}$ & 0.683 \\
\hline${ }^{15} 5 \mathrm{Eu}_{2} \mathrm{O}_{3}$ & $3.0 \mathrm{E}-12$ & $\mathrm{MgO}$ & $1.9 \mathrm{E}-05$ & $\mathrm{NiO}$ & 3.6E-05 \\
\hline${ }^{226} \mathrm{RaO}$ & $3.2 \mathrm{E}-08$ & $\mathrm{MoO}_{3}$ & 3.3E-05 & $\mathrm{PbO}$ & 0.00005 \\
\hline${ }^{227} \mathrm{Ac}_{2} \mathrm{O}_{3}$ & 7.4E-15 & $\mathrm{Nd}_{2} \mathrm{O}_{3}$ & 1.3E-05 & $\mathrm{P}_{2} \mathrm{O}_{5}$ & 0.0149 \\
\hline${ }^{228} \mathrm{RaO}$ & $8.4 \mathrm{E}-13$ & $\mathrm{PdO}$ & 2.6E-09 & $\mathrm{SiO}_{2}$ & 0.0090 \\
\hline${ }^{229} \mathrm{ThO}_{2}$ & $7.6 \mathrm{E}-12$ & $\mathrm{Pr}_{2} \mathrm{O}_{3}$ & 3.3E-09 & $\mathrm{SO}_{3}$ & 0.0128 \\
\hline${ }^{231} \mathrm{~Pa}_{2} \mathrm{O}_{5}$ & $3.1 \mathrm{E}-11$ & $\mathrm{Rb}_{2} \mathrm{O}$ & $1.8 \mathrm{E}-08$ & $\mathrm{SrO}$ & 1.4E-05 \\
\hline${ }^{232} \mathrm{ThO}_{2}$ & $3.6 \mathrm{E}-05$ & $\mathrm{Rh}_{2} \mathrm{O}_{3}$ & $3.0 \mathrm{E}-08$ & $\mathrm{ZrO}_{2}$ & 3.3E-05 \\
\hline${ }^{232} \mathrm{UO}_{3}$ & $2.1 \mathrm{E}-13$ & $\mathrm{RuO}_{2}$ & $1.4 \mathrm{E}-07$ & \multirow{26}{*}{\multicolumn{2}{|c|}{$\begin{array}{l}\text { Note that the oxide weight } \\
\text { fractions reported above } \\
\text { and immediately to the left } \\
\text { are for stable isotopes } \\
\text { alone. }\end{array}$}} \\
\hline${ }^{233} \mathrm{UO}_{3}$ & $2.4 \mathrm{E}-09$ & $\mathrm{Sb}_{2} \mathrm{O}_{3}$ & $1.2 \mathrm{E}-05$ & & \\
\hline${ }^{234} \mathrm{UO}_{3}$ & $4.4 \mathrm{E}-09$ & $\mathrm{SeO}_{2}$ & 1.3E-04 & & \\
\hline${ }^{235} \mathrm{UO}_{3}$ & $5.8 \mathrm{E}-07$ & $\mathrm{Ta}_{2} \mathrm{O}_{5}$ & 1.5E-09 & & \\
\hline${ }^{236} \mathrm{UO}_{3}$ & $1.4 \mathrm{E}-08$ & $\mathrm{TeO}_{2}$ & $4.2 \mathrm{E}-09$ & & \\
\hline${ }^{237} \mathrm{NpO}_{2}$ & $1.9 \mathrm{E}-07$ & $\mathrm{ThO}_{2}$ & 2.6E-08 & & \\
\hline${ }^{238} \mathrm{PuO}_{2}$ & $8.2 \mathrm{E}-12$ & $\mathrm{TiO}_{2}$ & $2.1 \mathrm{E}-06$ & & \\
\hline${ }^{238} \mathrm{UO}_{3}$ & $1.0 \mathrm{E}-04$ & $\mathrm{Tl}_{2} \mathrm{O}$ & 3.6E-05 & & \\
\hline${ }^{239} \mathrm{PuO}_{2}$ & $8.4 \mathrm{E}-08$ & $\mathrm{~V}_{2} \mathrm{O}_{5}$ & $9.8 \mathrm{E}-06$ & & \\
\hline${ }^{240} \mathrm{PuO}_{2}$ & 3.7E-09 & $\mathrm{WO}_{3}$ & 3.9E-07 & & \\
\hline${ }^{241} \mathrm{Am}_{2} \mathrm{O}_{3}$ & $3.0 \mathrm{E}-09$ & $\mathrm{Y}_{2} \mathrm{O}_{3}$ & $8.4 \mathrm{E}-08$ & & \\
\hline${ }^{241} \mathrm{PuO}_{2}$ & $2.7 \mathrm{E}-11$ & $\mathrm{ZnO}$ & 3.2E-06 & & \\
\hline${ }^{242} \mathrm{Cm}_{2} \mathrm{O}_{3}$ & $3.2 \mathrm{E}-14$ & & & & \\
\hline${ }^{242} \mathrm{PuO}_{2}$ & $1.2 \mathrm{E}-11$ & & & & \\
\hline${ }^{243} \mathrm{Am}_{2} \mathrm{O}_{3}$ & 2.2E-12 & & & & \\
\hline${ }^{243} \mathrm{Cm}_{2} \mathrm{O}_{3}$ & $1.1 \mathrm{E}-13$ & & & & \\
\hline${ }^{244} \mathrm{Cm}_{2} \mathrm{O}_{3}$ & 7.3E-13 & & & & \\
\hline${ }^{59} \mathrm{NiO}$ & 5.2E-09 & & & & \\
\hline${ }^{60} \mathrm{CoO}$ & 4.3E-13 & & & & \\
\hline${ }^{63} \mathrm{NiO}$ & $6.2 \mathrm{E}-10$ & & & & \\
\hline${ }^{79} \mathrm{SeO}_{2}$ & 9.9E-08 & & & & \\
\hline${ }^{90} \mathrm{SrO}$ & $2.8 \mathrm{E}-07$ & & & & \\
\hline${ }^{90} \mathrm{Y}_{2} \mathrm{O}_{3}$ & $2.2 \mathrm{E}-09$ & & & & \\
\hline${ }^{93} \mathrm{ZrO}_{2}$ & $2.5 \mathrm{E}-06$ & & & & \\
\hline${ }^{93} \mathrm{Nb}_{2} \mathrm{O}_{5}$ & $2.6 \mathrm{E}-11$ & & & & \\
\hline${ }^{99} \mathrm{Tc}_{2} \mathrm{O}_{7}$ & $2.2 \mathrm{E}-05$ & & & & \\
\hline
\end{tabular}


Table 5.11. Cluster 11 Waste Oxide Composition

\begin{tabular}{|c|c|c|c|c|c|}
\hline $\begin{array}{c}\text { Radioactive } \\
\text { oxides }\end{array}$ & Wt. frac. & $\begin{array}{l}\text { Supplemental } \\
\text { Species Oxides }\end{array}$ & Wt. frac. & $\begin{array}{c}\text { BBI Species } \\
\text { Oxides }\end{array}$ & Wt. frac. \\
\hline${ }^{106} \mathrm{RuO}_{2}$ & $0.0 \mathrm{E}+00$ & $\mathrm{Ag}_{2} \mathrm{O}$ & $5.5 \mathrm{E}-07$ & $\mathrm{Al}_{2} \mathrm{O}_{3}$ & 0.208 \\
\hline${ }^{113} \mathrm{CdO}$ & $4.8 \mathrm{E}-11$ & $\mathrm{As}_{2} \mathrm{O}_{5}$ & $3.0 \mathrm{E}-06$ & $\mathrm{Bi}_{2} \mathrm{O}_{3}$ & $2.1 \mathrm{E}-05$ \\
\hline${ }^{12} 5 \mathrm{Sb}_{2} \mathrm{O}_{3}$ & $5.2 \mathrm{E}-14$ & $\mathrm{~B}_{2} \mathrm{O}_{3}$ & $1.7 \mathrm{E}-05$ & $\mathrm{CaO}$ & 0.00023 \\
\hline${ }^{126} \mathrm{SnO}_{2}$ & 2.5E-08 & $\mathrm{BaO}$ & 6.4E-07 & $\mathrm{Cl}$ & 0.008 \\
\hline${ }^{129} \mathrm{I}$ & $4.4 \mathrm{E}-06$ & $\mathrm{BeO}$ & $2.6 \mathrm{E}-07$ & $\mathrm{Cr}_{2} \mathrm{O}_{3}$ & 0.0095 \\
\hline${ }^{134} \mathrm{Cs}_{2} \mathrm{O}$ & $6.6 \mathrm{E}-20$ & $\mathrm{CdO}$ & $1.0 \mathrm{E}-06$ & $\mathrm{~F}$ & 0.0097 \\
\hline${ }^{137} \mathrm{Cs}_{2} \mathrm{O}$ & $2.5 \mathrm{E}-10$ & $\mathrm{Ce}_{2} \mathrm{O}_{3}$ & $1.1 \mathrm{E}-03$ & $\mathrm{Fe}_{2} \mathrm{O}_{3}$ & 0.00028 \\
\hline${ }^{137} \mathrm{BaO}$ & $2.0 \mathrm{E}-13$ & $\mathrm{Co}_{2} \mathrm{O}_{3}$ & $8.2 \mathrm{E}-07$ & $\mathrm{~K}_{2} \mathrm{O}$ & 0.003 \\
\hline${ }^{151} \mathrm{Sm}_{2} \mathrm{O}_{3}$ & $3.3 \mathrm{E}-08$ & $\mathrm{Cs}_{2} \mathrm{O}$ & 1.4E-09 & $\mathrm{La}_{2} \mathrm{O}_{3}$ & $4.5 \mathrm{E}-07$ \\
\hline${ }^{152} \mathrm{Eu}_{2} \mathrm{O}_{3}$ & $6.9 \mathrm{E}-13$ & $\mathrm{CuO}$ & $7.2 \mathrm{E}-07$ & $\mathrm{MnO}$ & $1.2 \mathrm{E}-05$ \\
\hline${ }^{154} \mathrm{Eu}_{2} \mathrm{O}_{3}$ & $1.1 \mathrm{E}-11$ & $\mathrm{Li}_{2} \mathrm{O}$ & $4.8 \mathrm{E}-07$ & $\mathrm{Na}_{2} \mathrm{O}$ & 0.686 \\
\hline${ }^{15} 5 \mathrm{Eu}_{2} \mathrm{O}_{3}$ & $8.9 \mathrm{E}-13$ & $\mathrm{MgO}$ & $3.8 \mathrm{E}-06$ & $\mathrm{NiO}$ & 2.9E-05 \\
\hline${ }^{226} \mathrm{RaO}$ & $4.6 \mathrm{E}-09$ & $\mathrm{MoO}_{3}$ & $1.2 \mathrm{E}-05$ & $\mathrm{PbO}$ & 0.00002 \\
\hline${ }^{227} \mathrm{Ac}_{2} \mathrm{O}_{3}$ & $3.2 \mathrm{E}-15$ & $\mathrm{Nd}_{2} \mathrm{O}_{3}$ & $1.9 \mathrm{E}-06$ & $\mathrm{P}_{2} \mathrm{O}_{5}$ & 0.0302 \\
\hline${ }^{228} \mathrm{RaO}$ & $4.4 \mathrm{E}-13$ & $\mathrm{PdO}$ & 1.7E-09 & $\mathrm{SiO}_{2}$ & 0.0077 \\
\hline${ }^{229} \mathrm{ThO}_{2}$ & $2.4 \mathrm{E}-12$ & $\mathrm{Pr}_{2} \mathrm{O}_{3}$ & $5.5 \mathrm{E}-10$ & $\mathrm{SO}_{3}$ & 0.0355 \\
\hline${ }^{231} \mathrm{~Pa}_{2} \mathrm{O}_{5}$ & $2.6 \mathrm{E}-11$ & $\mathrm{Rb}_{2} \mathrm{O}$ & $3.4 \mathrm{E}-09$ & $\mathrm{SrO}$ & $2.2 \mathrm{E}-05$ \\
\hline${ }^{232} \mathrm{ThO}_{2}$ & $1.7 \mathrm{E}-05$ & $\mathrm{Rh}_{2} \mathrm{O}_{3}$ & 5.4E-09 & $\mathrm{ZrO}_{2}$ & 0.00012 \\
\hline${ }^{232} \mathrm{UO}_{3}$ & $2.8 \mathrm{E}-13$ & $\mathrm{RuO}_{2}$ & $4.5 \mathrm{E}-08$ & \multirow{26}{*}{\multicolumn{2}{|c|}{$\begin{array}{l}\text { Note that the oxide weight } \\
\text { fractions reported above } \\
\text { and immediately to the left } \\
\text { are for stable isotopes } \\
\text { alone. }\end{array}$}} \\
\hline${ }^{233} \mathrm{UO}_{3}$ & 3.3E-09 & $\mathrm{Sb}_{2} \mathrm{O}_{3}$ & 3.7E-06 & & \\
\hline${ }^{234} \mathrm{UO}_{3}$ & 7.9E-09 & $\mathrm{SeO}_{2}$ & 7.3E-05 & & \\
\hline${ }^{235} \mathrm{UO}_{3}$ & $9.8 \mathrm{E}-07$ & $\mathrm{Ta}_{2} \mathrm{O}_{5}$ & $1.2 \mathrm{E}-09$ & & \\
\hline${ }^{236} \mathrm{UO}_{3}$ & $2.7 \mathrm{E}-08$ & $\mathrm{TeO}_{2}$ & $1.0 \mathrm{E}-09$ & & \\
\hline${ }^{237} \mathrm{NpO}_{2}$ & 2.6E-07 & $\mathrm{ThO}_{2}$ & $1.1 \mathrm{E}-08$ & & \\
\hline${ }^{238} \mathrm{PuO}_{2}$ & 9.7E-11 & $\mathrm{TiO}_{2}$ & $2.7 \mathrm{E}-07$ & & \\
\hline${ }^{238} \mathrm{UO}_{3}$ & $1.4 \mathrm{E}-04$ & $\mathrm{Tl}_{2} \mathrm{O}$ & $3.2 \mathrm{E}-06$ & & \\
\hline${ }^{239} \mathrm{PuO}_{2}$ & 3.2E-07 & $\mathrm{V}_{2} \mathrm{O}_{5}$ & $1.4 \mathrm{E}-06$ & & \\
\hline${ }^{240} \mathrm{PuO}_{2}$ & 1.4E-08 & $\mathrm{WO}_{3}$ & $1.5 \mathrm{E}-07$ & & \\
\hline${ }^{241} \mathrm{Am}_{2} \mathrm{O}_{3}$ & $2.8 \mathrm{E}-09$ & $\mathrm{Y}_{2} \mathrm{O}_{3}$ & 3.5E-08 & & \\
\hline${ }^{241} \mathrm{PuO}_{2}$ & $8.0 \mathrm{E}-11$ & $\mathrm{ZnO}$ & $2.1 \mathrm{E}-06$ & & \\
\hline${ }^{242} \mathrm{Cm}_{2} \mathrm{O}_{3}$ & $1.6 \mathrm{E}-14$ & & & & \\
\hline${ }^{242} \mathrm{PuO}_{2}$ & 4.4E-11 & & & & \\
\hline${ }^{243} \mathrm{Am}_{2} \mathrm{O}_{3}$ & $1.5 \mathrm{E}-12$ & & & & \\
\hline${ }^{243} \mathrm{Cm}_{2} \mathrm{O}_{3}$ & $4.2 \mathrm{E}-14$ & & & & \\
\hline${ }^{244} \mathrm{Cm}_{2} \mathrm{O}_{3}$ & $3.4 \mathrm{E}-13$ & & & & \\
\hline${ }^{59} \mathrm{NiO}$ & $8.1 \mathrm{E}-09$ & & & & \\
\hline${ }^{60} \mathrm{CoO}$ & $1.7 \mathrm{E}-13$ & & & & \\
\hline${ }^{63} \mathrm{NiO}$ & $9.4 \mathrm{E}-10$ & & & & \\
\hline${ }^{79} \mathrm{SeO}_{2}$ & $1.0 \mathrm{E}-07$ & & & & \\
\hline${ }^{90} \mathrm{SrO}$ & $1.7 \mathrm{E}-07$ & & & & \\
\hline${ }^{90} \mathrm{Y}_{2} \mathrm{O}_{3}$ & $1.3 \mathrm{E}-09$ & & & & \\
\hline${ }^{93} \mathrm{ZrO}_{2}$ & $2.5 \mathrm{E}-06$ & & & & \\
\hline${ }^{93} \mathrm{Nb}_{2} \mathrm{O}_{5}$ & $2.1 \mathrm{E}-11$ & & & & \\
\hline${ }^{99} \mathrm{Tc}_{2} \mathrm{O}_{7}$ & $1.6 \mathrm{E}-05$ & & & & \\
\hline
\end{tabular}


Table 5.12. Cluster 12 Waste Oxide Composition

\begin{tabular}{|c|c|c|c|c|c|}
\hline $\begin{array}{c}\text { Radioactive } \\
\text { oxides }\end{array}$ & Wt. frac. & $\begin{array}{l}\text { Supplemental } \\
\text { Species Oxides }\end{array}$ & Wt. frac. & $\begin{array}{c}\text { BBI Species } \\
\text { Oxides }\end{array}$ & Wt. frac. \\
\hline${ }^{106} \mathrm{RuO}_{2}$ & $1.1 \mathrm{E}-17$ & $\mathrm{Ag}_{2} \mathrm{O}$ & $8.2 \mathrm{E}-07$ & $\mathrm{Al}_{2} \mathrm{O}_{3}$ & 0.091 \\
\hline${ }^{113} \mathrm{CdO}$ & $1.3 \mathrm{E}-10$ & $\mathrm{As}_{2} \mathrm{O}_{5}$ & 4.3E-06 & $\mathrm{Bi}_{2} \mathrm{O}_{3}$ & $8.2 \mathrm{E}-05$ \\
\hline${ }^{12} 5 \mathrm{Sb}_{2} \mathrm{O}_{3}$ & $1.4 \mathrm{E}-13$ & $\mathrm{~B}_{2} \mathrm{O}_{3}$ & $1.5 \mathrm{E}-05$ & $\mathrm{CaO}$ & 0.00060 \\
\hline${ }^{126} \mathrm{SnO}_{2}$ & 5.3E-08 & $\mathrm{BaO}$ & $1.9 \mathrm{E}-06$ & $\mathrm{Cl}$ & 0.008 \\
\hline${ }^{129} \mathrm{I}$ & $5.4 \mathrm{E}-06$ & $\mathrm{BeO}$ & $5.2 \mathrm{E}-07$ & $\mathrm{Cr}_{2} \mathrm{O}_{3}$ & 0.0057 \\
\hline${ }^{134} \mathrm{Cs}_{2} \mathrm{O}$ & 1.6E-19 & $\mathrm{CdO}$ & 3.9E-06 & $\mathrm{F}$ & 0.0117 \\
\hline${ }^{137} \mathrm{Cs}_{2} \mathrm{O}$ & $3.0 \mathrm{E}-10$ & $\mathrm{Ce}_{2} \mathrm{O}_{3}$ & $8.0 \mathrm{E}-04$ & $\mathrm{Fe}_{2} \mathrm{O}_{3}$ & 0.00045 \\
\hline${ }^{137} \mathrm{BaO}$ & $2.5 \mathrm{E}-13$ & $\mathrm{Co}_{2} \mathrm{O}_{3}$ & 1.5E-06 & $\mathrm{K}_{2} \mathrm{O}$ & 0.005 \\
\hline${ }^{151} \mathrm{Sm}_{2} \mathrm{O}_{3}$ & $3.5 \mathrm{E}-08$ & $\mathrm{Cs}_{2} \mathrm{O}$ & $1.5 \mathrm{E}-09$ & $\mathrm{La}_{2} \mathrm{O}_{3}$ & $1.6 \mathrm{E}-06$ \\
\hline${ }^{152} \mathrm{Eu}_{2} \mathrm{O}_{3}$ & $1.2 \mathrm{E}-12$ & $\mathrm{CuO}$ & 1.5E-06 & $\mathrm{MnO}$ & $3.7 \mathrm{E}-05$ \\
\hline${ }^{154} \mathrm{Eu}_{2} \mathrm{O}_{3}$ & 4.7E-11 & $\mathrm{Li}_{2} \mathrm{O}$ & $1.2 \mathrm{E}-06$ & $\mathrm{Na}_{2} \mathrm{O}$ & 0.761 \\
\hline${ }^{15} 5 \mathrm{Eu}_{2} \mathrm{O}_{3}$ & $4.4 \mathrm{E}-12$ & $\mathrm{MgO}$ & $4.4 \mathrm{E}-06$ & $\mathrm{NiO}$ & $1.0 \mathrm{E}-04$ \\
\hline${ }^{226} \mathrm{RaO}$ & $1.1 \mathrm{E}-09$ & $\mathrm{MoO}_{3}$ & $1.2 \mathrm{E}-05$ & $\mathrm{PbO}$ & 0.00008 \\
\hline${ }^{227} \mathrm{Ac}_{2} \mathrm{O}_{3}$ & 2.3E-15 & $\mathrm{Nd}_{2} \mathrm{O}_{3}$ & $5.1 \mathrm{E}-06$ & $\mathrm{P}_{2} \mathrm{O}_{5}$ & 0.0588 \\
\hline${ }^{228} \mathrm{RaO}$ & $7.4 \mathrm{E}-13$ & PdO & 3.3E-09 & $\mathrm{SiO}_{2}$ & 0.0091 \\
\hline${ }^{229} \mathrm{ThO}_{2}$ & $1.1 \mathrm{E}-11$ & $\mathrm{Pr}_{2} \mathrm{O}_{3}$ & 4.3E-09 & $\mathrm{SO}_{3}$ & 0.0464 \\
\hline${ }^{231} \mathrm{~Pa}_{2} \mathrm{O}_{5}$ & $4.5 \mathrm{E}-11$ & $\mathrm{Rb}_{2} \mathrm{O}$ & $2.1 \mathrm{E}-08$ & $\mathrm{SrO}$ & $3.4 \mathrm{E}-05$ \\
\hline${ }^{232} \mathrm{ThO}_{2}$ & 5.9E-05 & $\mathrm{Rh}_{2} \mathrm{O}_{3}$ & $7.9 \mathrm{E}-07$ & $\mathrm{ZrO}_{2}$ & $9.8 \mathrm{E}-05$ \\
\hline${ }^{232} \mathrm{UO}_{3}$ & $3.7 \mathrm{E}-13$ & $\mathrm{RuO}_{2}$ & $1.7 \mathrm{E}-07$ & \multirow{26}{*}{\multicolumn{2}{|c|}{$\begin{array}{l}\text { Note that the oxide weight } \\
\text { fractions reported above } \\
\text { and immediately to the left } \\
\text { are for stable isotopes } \\
\text { alone. }\end{array}$}} \\
\hline${ }^{233} \mathrm{UO}_{3}$ & 4.3E-09 & $\mathrm{Sb}_{2} \mathrm{O}_{3}$ & $1.9 \mathrm{E}-05$ & & \\
\hline${ }^{234} \mathrm{UO}_{3}$ & $9.0 \mathrm{E}-09$ & $\mathrm{SeO}_{2}$ & $1.1 \mathrm{E}-04$ & & \\
\hline${ }^{235} \mathrm{UO}_{3}$ & $1.1 \mathrm{E}-06$ & $\mathrm{Ta}_{2} \mathrm{O}_{5}$ & $6.7 \mathrm{E}-07$ & & \\
\hline${ }^{236} \mathrm{UO}_{3}$ & $2.2 \mathrm{E}-08$ & $\mathrm{TeO}_{2}$ & $5.8 \mathrm{E}-09$ & & \\
\hline${ }^{237} \mathrm{NpO}_{2}$ & 2.7E-07 & $\mathrm{ThO}_{2}$ & $4.6 \mathrm{E}-08$ & & \\
\hline${ }^{238} \mathrm{PuO}_{2}$ & $1.4 \mathrm{E}-10$ & $\mathrm{TiO}_{2}$ & $4.8 \mathrm{E}-07$ & & \\
\hline${ }^{238} \mathrm{UO}_{3}$ & $1.9 \mathrm{E}-04$ & $\mathrm{Tl}_{2} \mathrm{O}$ & $2.6 \mathrm{E}-06$ & & \\
\hline${ }^{239} \mathrm{PuO}_{2}$ & 5.1E-07 & $\mathrm{V}_{2} \mathrm{O}_{5}$ & $2.0 \mathrm{E}-06$ & & \\
\hline${ }^{240} \mathrm{PuO}_{2}$ & 2.2E-08 & $\mathrm{WO}_{3}$ & $4.6 \mathrm{E}-07$ & & \\
\hline${ }^{241} \mathrm{Am}_{2} \mathrm{O}_{3}$ & $1.5 \mathrm{E}-08$ & $\mathrm{Y}_{2} \mathrm{O}_{3}$ & 3.7E-07 & & \\
\hline${ }^{241} \mathrm{PuO}_{2}$ & $1.2 \mathrm{E}-10$ & $\mathrm{ZnO}$ & $1.3 \mathrm{E}-06$ & & \\
\hline${ }^{242} \mathrm{Cm}_{2} \mathrm{O}_{3}$ & 5.3E-14 & & & & \\
\hline${ }^{242} \mathrm{PuO}_{2}$ & 6.7E-11 & & & & \\
\hline${ }^{243} \mathrm{Am}_{2} \mathrm{O}_{3}$ & $8.1 \mathrm{E}-12$ & & & & \\
\hline${ }^{243} \mathrm{Cm}_{2} \mathrm{O}_{3}$ & $1.8 \mathrm{E}-13$ & & & & \\
\hline${ }^{244} \mathrm{Cm}_{2} \mathrm{O}_{3}$ & $1.1 \mathrm{E}-12$ & & & & \\
\hline${ }^{59} \mathrm{NiO}$ & 9.4E-09 & & & & \\
\hline${ }^{60} \mathrm{CoO}$ & $7.8 \mathrm{E}-13$ & & & & \\
\hline${ }^{63} \mathrm{NiO}$ & $1.1 \mathrm{E}-09$ & & & & \\
\hline${ }^{79} \mathrm{SeO}_{2}$ & $1.8 \mathrm{E}-07$ & & & & \\
\hline${ }^{90} \mathrm{SrO}$ & $1.6 \mathrm{E}-06$ & & & & \\
\hline${ }^{90} \mathrm{Y}_{2} \mathrm{O}_{3}$ & $2.1 \mathrm{E}-09$ & & & & \\
\hline${ }^{93} \mathrm{ZrO}_{2}$ & 5.3E-06 & & & & \\
\hline${ }^{93} \mathrm{Nb}_{2} \mathrm{O}_{5}$ & 4.3E-11 & & & & \\
\hline${ }^{99} \mathrm{Tc}_{2} \mathrm{O}_{7}$ & $1.9 \mathrm{E}-05$ & & & & \\
\hline
\end{tabular}


Table 5.13. Cluster 13 Waste Oxide Composition

\begin{tabular}{|c|c|c|c|c|c|}
\hline $\begin{array}{c}\text { Radioactive } \\
\text { oxides }\end{array}$ & Wt. frac. & $\begin{array}{l}\text { Supplemental } \\
\text { Species Oxides }\end{array}$ & $\begin{array}{l}\text { Wt. } \\
\text { frac. }\end{array}$ & $\begin{array}{c}\text { BBI Species } \\
\text { Oxides }\end{array}$ & Wt. frac. \\
\hline${ }^{106} \mathrm{RuO}_{2}$ & $1.9 \mathrm{E}-18$ & $\mathrm{Ag}_{2} \mathrm{O}$ & $1.9 \mathrm{E}-07$ & $\mathrm{Al}_{2} \mathrm{O}_{3}$ & 0.078 \\
\hline${ }^{113} \mathrm{CdO}$ & $1.2 \mathrm{E}-10$ & $\mathrm{As}_{2} \mathrm{O}_{5}$ & $9.0 \mathrm{E}-07$ & $\mathrm{Bi}_{2} \mathrm{O}_{3}$ & $2.8 \mathrm{E}-05$ \\
\hline${ }^{12} 5 \mathrm{Sb}_{2} \mathrm{O}_{3}$ & 4.0E-14 & $\mathrm{B}_{2} \mathrm{O}_{3}$ & 2.4E-06 & $\mathrm{CaO}$ & 0.00029 \\
\hline${ }^{126} \mathrm{SnO}_{2}$ & $1.7 \mathrm{E}-08$ & $\mathrm{BaO}$ & 4.0E-07 & $\mathrm{Cl}$ & 0.007 \\
\hline${ }^{129} \mathrm{I}$ & 5.2E-06 & $\mathrm{BeO}$ & $1.1 \mathrm{E}-07$ & $\mathrm{Cr}_{2} \mathrm{O}_{3}$ & 0.0051 \\
\hline${ }^{134} \mathrm{Cs}_{2} \mathrm{O}$ & $0.0 \mathrm{E}+00$ & $\mathrm{CdO}$ & 1.5E-06 & $\mathrm{F}$ & 0.0112 \\
\hline${ }^{137} \mathrm{Cs}_{2} \mathrm{O}$ & $1.8 \mathrm{E}-10$ & $\mathrm{Ce}_{2} \mathrm{O}_{3}$ & 8.1E-04 & $\mathrm{Fe}_{2} \mathrm{O}_{3}$ & 0.00059 \\
\hline${ }^{137} \mathrm{BaO}$ & $1.5 \mathrm{E}-13$ & $\mathrm{Co}_{2} \mathrm{O}_{3}$ & 4.3E-07 & $\mathrm{K}_{2} \mathrm{O}$ & 0.003 \\
\hline${ }^{151} \mathrm{Sm}_{2} \mathrm{O}_{3}$ & 1.4E-08 & $\mathrm{Cs}_{2} \mathrm{O}$ & $9.9 \mathrm{E}-10$ & $\mathrm{La}_{2} \mathrm{O}_{3}$ & $2.9 \mathrm{E}-07$ \\
\hline${ }^{152} \mathrm{Eu}_{2} \mathrm{O}_{3}$ & $2.6 \mathrm{E}-13$ & $\mathrm{CuO}$ & 1.6E-07 & $\mathrm{MnO}$ & $1.7 \mathrm{E}-05$ \\
\hline${ }^{154} \mathrm{Eu}_{2} \mathrm{O}_{3}$ & 8.9E-12 & $\mathrm{Li}_{2} \mathrm{O}$ & 3.3E-07 & $\mathrm{Na}_{2} \mathrm{O}$ & 0.765 \\
\hline${ }^{15} 5 \mathrm{Eu}_{2} \mathrm{O}_{3}$ & $4.0 \mathrm{E}-13$ & $\mathrm{MgO}$ & $1.1 \mathrm{E}-06$ & $\mathrm{NiO}$ & $7.9 \mathrm{E}-05$ \\
\hline${ }^{226} \mathrm{RaO}$ & 2.9E-10 & $\mathrm{MoO}_{3}$ & 2.0E-06 & $\mathrm{PbO}$ & 0.00003 \\
\hline${ }^{227} \mathrm{Ac}_{2} \mathrm{O}_{3}$ & 7.4E-16 & $\mathrm{Nd}_{2} \mathrm{O}_{3}$ & 7.1E-07 & $\mathrm{P}_{2} \mathrm{O}_{5}$ & 0.0456 \\
\hline${ }^{228} \mathrm{RaO}$ & $8.0 \mathrm{E}-13$ & $\mathrm{PdO}$ & $4.2 \mathrm{E}-10$ & $\mathrm{SiO}_{2}$ & 0.0068 \\
\hline${ }^{229} \mathrm{ThO}_{2}$ & 4.2E-12 & $\mathrm{Pr}_{2} \mathrm{O}_{3}$ & 4.9E-10 & $\mathrm{SO}_{3}$ & 0.0763 \\
\hline${ }^{231} \mathrm{~Pa}_{2} \mathrm{O}_{5}$ & $2.7 \mathrm{E}-11$ & $\mathrm{Rb}_{2} \mathrm{O}$ & $2.1 \mathrm{E}-09$ & $\mathrm{SrO}$ & $3.6 \mathrm{E}-05$ \\
\hline${ }^{232} \mathrm{ThO}_{2}$ & 2.2E-05 & $\mathrm{Rh}_{2} \mathrm{O}_{3}$ & 4.1E-08 & $\mathrm{ZrO}_{2}$ & $2.8 \mathrm{E}-05$ \\
\hline${ }^{232} \mathrm{UO}_{3}$ & $2.2 \mathrm{E}-13$ & $\mathrm{RuO}_{2}$ & 5.2E-08 & \multirow{26}{*}{\multicolumn{2}{|c|}{$\begin{array}{l}\text { Note that the oxide weight } \\
\text { fractions reported above } \\
\text { and immediately to the left } \\
\text { are for stable isotopes } \\
\text { alone. }\end{array}$}} \\
\hline${ }^{233} \mathrm{UO}_{3}$ & 2.5E-09 & $\mathrm{Sb}_{2} \mathrm{O}_{3}$ & $2.8 \mathrm{E}-06$ & & \\
\hline${ }^{234} \mathrm{UO}_{3}$ & 4.7E-09 & $\mathrm{SeO}_{2}$ & $3.7 \mathrm{E}-05$ & & \\
\hline${ }^{235} \mathrm{UO}_{3}$ & 5.8E-07 & $\mathrm{Ta}_{2} \mathrm{O}_{5}$ & 3.2E-08 & & \\
\hline${ }^{236} \mathrm{UO}_{3}$ & $9.5 \mathrm{E}-09$ & $\mathrm{TeO}_{2}$ & $1.4 \mathrm{E}-09$ & & \\
\hline${ }^{237} \mathrm{NpO}_{2}$ & 3.0E-07 & $\mathrm{ThO}_{2}$ & $1.5 \mathrm{E}-09$ & & \\
\hline${ }^{238} \mathrm{PuO}_{2}$ & $1.9 \mathrm{E}-11$ & $\mathrm{TiO}_{2}$ & $1.2 \mathrm{E}-07$ & & \\
\hline${ }^{238} \mathrm{UO}_{3}$ & 8.9E-05 & $\mathrm{Tl}_{2} \mathrm{O}$ & 1.9E-07 & & \\
\hline${ }^{239} \mathrm{PuO}_{2}$ & $2.1 \mathrm{E}-07$ & $\mathrm{~V}_{2} \mathrm{O}_{5}$ & 6.6E-07 & & \\
\hline${ }^{240} \mathrm{PuO}_{2}$ & 9.0E-09 & $\mathrm{WO}_{3}$ & 5.0E-08 & & \\
\hline${ }^{241} \mathrm{Am}_{2} \mathrm{O}_{3}$ & 1.8E-09 & $\mathrm{Y}_{2} \mathrm{O}_{3}$ & $2.5 \mathrm{E}-08$ & & \\
\hline${ }^{241} \mathrm{PuO}_{2}$ & 5.3E-11 & $\mathrm{ZnO}$ & 2.3E-07 & & \\
\hline${ }^{242} \mathrm{Cm}_{2} \mathrm{O}_{3}$ & 5.0E-15 & & & & \\
\hline${ }^{242} \mathrm{PuO}_{2}$ & 2.9E-11 & & & & \\
\hline${ }^{243} \mathrm{Am}_{2} \mathrm{O}_{3}$ & $1.0 \mathrm{E}-12$ & & & & \\
\hline${ }^{243} \mathrm{Cm}_{2} \mathrm{O}_{3}$ & $2.1 \mathrm{E}-14$ & & & & \\
\hline${ }^{244} \mathrm{Cm}_{2} \mathrm{O}_{3}$ & $1.8 \mathrm{E}-13$ & & & & \\
\hline${ }^{59} \mathrm{NiO}$ & $2.1 \mathrm{E}-08$ & & & & \\
\hline${ }^{60} \mathrm{CoO}$ & 2.2E-13 & & & & \\
\hline${ }^{63} \mathrm{NiO}$ & 2.5E-09 & & & & \\
\hline${ }^{79} \mathrm{SeO}_{2}$ & $1.4 \mathrm{E}-07$ & & & & \\
\hline${ }^{90} \mathrm{SrO}$ & $1.5 \mathrm{E}-07$ & & & & \\
\hline${ }^{90} \mathrm{Y}_{2} \mathrm{O}_{3}$ & 6.3E-10 & & & & \\
\hline${ }^{93} \mathrm{ZrO}_{2}$ & 3.5E-06 & & & & \\
\hline${ }^{93} \mathrm{Nb}_{2} \mathrm{O}_{5}$ & 2.7E-11 & & & & \\
\hline${ }^{99} \mathrm{Tc}_{2} \mathrm{O}_{7}$ & $1.3 \mathrm{E}-05$ & & & & \\
\hline
\end{tabular}


Table 5.14. Cluster 14 Waste Oxide Composition

\begin{tabular}{|c|c|c|c|c|c|}
\hline $\begin{array}{c}\text { Radioactive } \\
\text { oxides }\end{array}$ & Wt. frac. & $\begin{array}{l}\text { Supplemental } \\
\text { Species Oxides }\end{array}$ & Wt. frac. & $\begin{array}{c}\text { BBI Species } \\
\text { Oxides }\end{array}$ & Wt. frac. \\
\hline${ }^{106} \mathrm{RuO}_{2}$ & 7.4E-17 & $\mathrm{Ag}_{2} \mathrm{O}$ & $1.1 \mathrm{E}-06$ & $\mathrm{Al}_{2} \mathrm{O}_{3}$ & 0.172 \\
\hline${ }^{113} \mathrm{CdO}$ & 7.2E-11 & $\mathrm{As}_{2} \mathrm{O}_{5}$ & 7.6E-06 & $\mathrm{Bi}_{2} \mathrm{O}_{3}$ & $9.7 \mathrm{E}-05$ \\
\hline${ }^{12} 5 \mathrm{Sb}_{2} \mathrm{O}_{3}$ & $6.8 \mathrm{E}-13$ & $\mathrm{~B}_{2} \mathrm{O}_{3}$ & 2.5E-05 & $\mathrm{CaO}$ & 0.00057 \\
\hline${ }^{126} \mathrm{SnO}_{2}$ & 4.3E-08 & $\mathrm{BaO}$ & $1.8 \mathrm{E}-06$ & $\mathrm{Cl}$ & 0.009 \\
\hline${ }^{129} \mathrm{I}$ & 4.7E-06 & $\mathrm{BeO}$ & 7.6E-07 & $\mathrm{Cr}_{2} \mathrm{O}_{3}$ & 0.0122 \\
\hline${ }^{134} \mathrm{Cs}_{2} \mathrm{O}$ & 7.5E-18 & $\mathrm{CdO}$ & 1.3E-05 & $\mathrm{F}$ & 0.0073 \\
\hline${ }^{137} \mathrm{Cs}_{2} \mathrm{O}$ & 3.3E-10 & $\mathrm{Ce}_{2} \mathrm{O}_{3}$ & 1.6E-03 & $\mathrm{Fe}_{2} \mathrm{O}_{3}$ & 0.00056 \\
\hline${ }^{137} \mathrm{BaO}$ & 2.6E-13 & $\mathrm{Co}_{2} \mathrm{O}_{3}$ & 5.2E-06 & $\mathrm{K}_{2} \mathrm{O}$ & 0.004 \\
\hline${ }^{151} \mathrm{Sm}_{2} \mathrm{O}_{3}$ & $5.4 \mathrm{E}-08$ & $\mathrm{Cs}_{2} \mathrm{O}$ & $1.5 \mathrm{E}-09$ & $\mathrm{La}_{2} \mathrm{O}_{3}$ & $2.3 \mathrm{E}-06$ \\
\hline${ }^{152} \mathrm{Eu}_{2} \mathrm{O}_{3}$ & 5.3E-12 & $\mathrm{CuO}$ & $1.0 \mathrm{E}-06$ & $\mathrm{MnO}$ & $2.0 \mathrm{E}-05$ \\
\hline${ }^{154} \mathrm{Eu}_{2} \mathrm{O}_{3}$ & 5.8E-11 & $\mathrm{Li}_{2} \mathrm{O}$ & $1.6 \mathrm{E}-06$ & $\mathrm{Na}_{2} \mathrm{O}$ & 0.705 \\
\hline${ }^{15} 5 \mathrm{Eu}_{2} \mathrm{O}_{3}$ & 6.2E-12 & $\mathrm{MgO}$ & 8.8E-06 & $\mathrm{NiO}$ & 6.0E-05 \\
\hline${ }^{226} \mathrm{RaO}$ & $1.9 \mathrm{E}-08$ & $\mathrm{MoO}_{3}$ & $1.9 \mathrm{E}-05$ & $\mathrm{PbO}$ & 0.00006 \\
\hline${ }^{227} \mathrm{Ac}_{2} \mathrm{O}_{3}$ & $3.8 \mathrm{E}-15$ & $\mathrm{Nd}_{2} \mathrm{O}_{3}$ & 6.0E-06 & $\mathrm{P}_{2} \mathrm{O}_{5}$ & 0.0482 \\
\hline${ }^{228} \mathrm{RaO}$ & 7.4E-13 & $\mathrm{PdO}$ & $3.4 \mathrm{E}-08$ & $\mathrm{SiO}_{2}$ & 0.0143 \\
\hline${ }^{229} \mathrm{ThO}_{2}$ & $2.5 \mathrm{E}-11$ & $\mathrm{Pr}_{2} \mathrm{O}_{3}$ & $1.8 \mathrm{E}-08$ & $\mathrm{SO}_{3}$ & 0.0247 \\
\hline${ }^{231} \mathrm{~Pa}_{2} \mathrm{O}_{5}$ & 3.5E-11 & $\mathrm{Rb}_{2} \mathrm{O}$ & $1.2 \mathrm{E}-07$ & $\mathrm{SrO}$ & $3.4 \mathrm{E}-05$ \\
\hline${ }^{232} \mathrm{ThO}_{2}$ & $2.5 \mathrm{E}-05$ & $\mathrm{Rh}_{2} \mathrm{O}_{3}$ & 3.3E-07 & $\mathrm{ZrO}_{2}$ & 7.5E-05 \\
\hline${ }^{232} \mathrm{UO}_{3}$ & $3.1 \mathrm{E}-13$ & $\mathrm{RuO}_{2}$ & $1.1 \mathrm{E}-06$ & \multirow{26}{*}{\multicolumn{2}{|c|}{$\begin{array}{l}\text { Note that the oxide weight } \\
\text { fractions reported above } \\
\text { and immediately to the left } \\
\text { are for stable isotopes } \\
\text { alone. }\end{array}$}} \\
\hline${ }^{233} \mathrm{UO}_{3}$ & $3.8 \mathrm{E}-09$ & $\mathrm{Sb}_{2} \mathrm{O}_{3}$ & $1.2 \mathrm{E}-05$ & & \\
\hline${ }^{234} \mathrm{UO}_{3}$ & 1.4E-08 & $\mathrm{SeO}_{2}$ & $1.9 \mathrm{E}-04$ & & \\
\hline${ }^{235} \mathrm{UO}_{3}$ & $2.0 \mathrm{E}-06$ & $\mathrm{Ta}_{2} \mathrm{O}_{5}$ & $1.0 \mathrm{E}-07$ & & \\
\hline${ }^{236} \mathrm{UO}_{3}$ & $3.2 \mathrm{E}-08$ & $\mathrm{TeO}_{2}$ & $2.4 \mathrm{E}-08$ & & \\
\hline${ }^{237} \mathrm{NpO}_{2}$ & $3.4 \mathrm{E}-07$ & $\mathrm{ThO}_{2}$ & 6.8E-08 & & \\
\hline${ }^{238} \mathrm{PuO}_{2}$ & 5.7E-11 & $\mathrm{TiO}_{2}$ & $9.5 \mathrm{E}-07$ & & \\
\hline${ }^{238} \mathrm{UO}_{3}$ & 4.5E-04 & $\mathrm{Tl}_{2} \mathrm{O}$ & $1.2 \mathrm{E}-05$ & & \\
\hline${ }^{239} \mathrm{PuO}_{2}$ & 2.7E-07 & $\mathrm{V}_{2} \mathrm{O}_{5}$ & 4.1E-06 & & \\
\hline${ }^{240} \mathrm{PuO}_{2}$ & $1.1 \mathrm{E}-08$ & $\mathrm{WO}_{3}$ & 3.1E-06 & & \\
\hline${ }^{241} \mathrm{Am}_{2} \mathrm{O}_{3}$ & $1.5 \mathrm{E}-08$ & $\mathrm{Y}_{2} \mathrm{O}_{3}$ & $6.2 \mathrm{E}-07$ & & \\
\hline${ }^{241} \mathrm{PuO}_{2}$ & 7.2E-11 & $\mathrm{ZnO}$ & $2.7 \mathrm{E}-06$ & & \\
\hline${ }^{242} \mathrm{Cm}_{2} \mathrm{O}_{3}$ & 3.3E-14 & & & & \\
\hline${ }^{242} \mathrm{PuO}_{2}$ & 3.5E-11 & & & & \\
\hline${ }^{243} \mathrm{Am}_{2} \mathrm{O}_{3}$ & 9.7E-12 & & & & \\
\hline${ }^{243} \mathrm{Cm}_{2} \mathrm{O}_{3}$ & $1.4 \mathrm{E}-13$ & & & & \\
\hline${ }^{244} \mathrm{Cm}_{2} \mathrm{O}_{3}$ & $1.0 \mathrm{E}-12$ & & & & \\
\hline${ }^{59} \mathrm{NiO}$ & 6.2E-09 & & & & \\
\hline${ }^{60} \mathrm{CoO}$ & $6.2 \mathrm{E}-13$ & & & & \\
\hline${ }^{63} \mathrm{NiO}$ & $7.3 \mathrm{E}-10$ & & & & \\
\hline${ }^{79} \mathrm{SeO}_{2}$ & $1.9 \mathrm{E}-07$ & & & & \\
\hline${ }^{90} \mathrm{SrO}$ & 1.3E-06 & & & & \\
\hline${ }^{90} \mathrm{Y}_{2} \mathrm{O}_{3}$ & 2.0E-09 & & & & \\
\hline${ }^{93} \mathrm{ZrO}_{2}$ & 5.4E-06 & & & & \\
\hline${ }^{93} \mathrm{Nb}_{2} \mathrm{O}_{5}$ & 4.7E-11 & & & & \\
\hline${ }^{99} \mathrm{Tc}_{2} \mathrm{O}_{7}$ & 2.2E-05 & & & & \\
\hline
\end{tabular}


Table 5.15. Cluster 15 Waste Oxide Composition

\begin{tabular}{|c|c|c|c|c|c|}
\hline $\begin{array}{c}\text { Radioactive } \\
\text { oxides }\end{array}$ & Wt. frac. & $\begin{array}{l}\text { Supplemental } \\
\text { Species Oxides }\end{array}$ & Wt. frac. & $\begin{array}{c}\text { BBI Species } \\
\text { Oxides }\end{array}$ & $\begin{array}{l}\text { Wt. } \\
\text { frac. }\end{array}$ \\
\hline${ }^{106} \mathrm{RuO}_{2}$ & $1.4 \mathrm{E}-18$ & $\mathrm{Ag}_{2} \mathrm{O}$ & 7.6E-06 & $\mathrm{Al}_{2} \mathrm{O}_{3}$ & 0.109 \\
\hline${ }^{113} \mathrm{CdO}$ & $2.0 \mathrm{E}-10$ & $\mathrm{As}_{2} \mathrm{O}_{5}$ & $6.0 \mathrm{E}-05$ & $\mathrm{Bi}_{2} \mathrm{O}_{3}$ & 5.9E-05 \\
\hline${ }^{12} 5 \mathrm{Sb}_{2} \mathrm{O}_{3}$ & $8.0 \mathrm{E}-12$ & $\mathrm{~B}_{2} \mathrm{O}_{3}$ & $1.9 \mathrm{E}-04$ & $\mathrm{CaO}$ & 0.00051 \\
\hline${ }^{126} \mathrm{SnO}_{2}$ & $5.0 \mathrm{E}-07$ & $\mathrm{BaO}$ & 2.0E-05 & $\mathrm{Cl}$ & 0.011 \\
\hline${ }^{129} \mathrm{I}$ & 7.1E-06 & $\mathrm{BeO}$ & $6.8 \mathrm{E}-06$ & $\mathrm{Cr}_{2} \mathrm{O}_{3}$ & 0.0047 \\
\hline${ }^{134} \mathrm{Cs}_{2} \mathrm{O}$ & 7.4E-16 & $\mathrm{CdO}$ & $3.9 \mathrm{E}-05$ & $F$ & 0.0064 \\
\hline${ }^{137} \mathrm{Cs}_{2} \mathrm{O}$ & 1.3E-09 & $\mathrm{Ce}_{2} \mathrm{O}_{3}$ & $8.0 \mathrm{E}-04$ & $\mathrm{Fe}_{2} \mathrm{O}_{3}$ & 0.00102 \\
\hline${ }^{137} \mathrm{BaO}$ & $8.5 \mathrm{E}-13$ & $\mathrm{Co}_{2} \mathrm{O}_{3}$ & $1.1 \mathrm{E}-05$ & $\mathrm{~K}_{2} \mathrm{O}$ & 0.025 \\
\hline${ }^{151} \mathrm{Sm}_{2} \mathrm{O}_{3}$ & $4.1 \mathrm{E}-07$ & $\mathrm{Cs}_{2} \mathrm{O}$ & 5.2E-09 & $\mathrm{La}_{2} \mathrm{O}_{3}$ & $1.9 \mathrm{E}-05$ \\
\hline${ }^{152} \mathrm{Eu}_{2} \mathrm{O}_{3}$ & $2.1 \mathrm{E}-11$ & $\mathrm{CuO}$ & $1.9 \mathrm{E}-05$ & $\mathrm{MnO}$ & $2.2 \mathrm{E}-04$ \\
\hline${ }^{154} \mathrm{Eu}_{2} \mathrm{O}_{3}$ & 5.3E-10 & $\mathrm{Li}_{2} \mathrm{O}$ & $1.1 \mathrm{E}-05$ & $\mathrm{Na}_{2} \mathrm{O}$ & 0.797 \\
\hline${ }^{15} 5 \mathrm{Eu}_{2} \mathrm{O}_{3}$ & $8.9 \mathrm{E}-11$ & $\mathrm{MgO}$ & 6.6E-05 & $\mathrm{NiO}$ & 3.6E-04 \\
\hline${ }^{226} \mathrm{RaO}$ & $1.0 \mathrm{E}-09$ & $\mathrm{MoO}_{3}$ & $1.4 \mathrm{E}-04$ & $\mathrm{PbO}$ & 0.00026 \\
\hline${ }^{227} \mathrm{Ac}_{2} \mathrm{O}_{3}$ & $3.7 \mathrm{E}-14$ & $\mathrm{Nd}_{2} \mathrm{O}_{3}$ & $6.8 \mathrm{E}-05$ & $\mathrm{P}_{2} \mathrm{O}_{5}$ & 0.0140 \\
\hline${ }^{228} \mathrm{RaO}$ & $4.2 \mathrm{E}-12$ & PdO & $3.0 \mathrm{E}-07$ & $\mathrm{SiO}_{2}$ & 0.0044 \\
\hline${ }^{229} \mathrm{ThO}_{2}$ & $2.4 \mathrm{E}-11$ & $\mathrm{Pr}_{2} \mathrm{O}_{3}$ & $7.8 \mathrm{E}-08$ & $\mathrm{SO}_{3}$ & 0.0238 \\
\hline${ }^{231} \mathrm{~Pa}_{2} \mathrm{O}_{5}$ & $1.5 \mathrm{E}-10$ & $\mathrm{Rb}_{2} \mathrm{O}$ & $2.2 \mathrm{E}-06$ & $\mathrm{SrO}$ & 9.0E-06 \\
\hline${ }^{232} \mathrm{ThO}_{2}$ & $1.9 \mathrm{E}-04$ & $\mathrm{Rh}_{2} \mathrm{O}_{3}$ & $1.0 \mathrm{E}-05$ & $\mathrm{ZrO}_{2}$ & 5.8E-05 \\
\hline${ }^{232} \mathrm{UO}_{3}$ & $1.9 \mathrm{E}-12$ & $\mathrm{RuO}_{2}$ & $1.2 \mathrm{E}-05$ & \multirow{26}{*}{\multicolumn{2}{|c|}{$\begin{array}{l}\text { Note that the oxide weight } \\
\text { fractions reported above } \\
\text { and immediately to the left } \\
\text { are for stable isotopes } \\
\text { alone. }\end{array}$}} \\
\hline${ }^{233} \mathrm{UO}_{3}$ & $2.2 \mathrm{E}-08$ & $\mathrm{Sb}_{2} \mathrm{O}_{3}$ & $3.5 \mathrm{E}-05$ & & \\
\hline${ }^{234} \mathrm{UO}_{3}$ & $2.1 \mathrm{E}-08$ & $\mathrm{SeO}_{2}$ & $3.6 \mathrm{E}-04$ & & \\
\hline${ }^{235} \mathrm{UO}_{3}$ & $2.6 \mathrm{E}-06$ & $\mathrm{Ta}_{2} \mathrm{O}_{5}$ & $8.3 \mathrm{E}-06$ & & \\
\hline${ }^{236} \mathrm{UO}_{3}$ & $6.0 \mathrm{E}-08$ & $\mathrm{TeO}_{2}$ & $3.1 \mathrm{E}-07$ & & \\
\hline${ }^{237} \mathrm{NpO}_{2}$ & $3.9 \mathrm{E}-06$ & $\mathrm{ThO}_{2}$ & $4.7 \mathrm{E}-08$ & & \\
\hline${ }^{238} \mathrm{PuO}_{2}$ & 5.1E-11 & $\mathrm{TiO}_{2}$ & 7.6E-06 & & \\
\hline${ }^{238} \mathrm{UO}_{3}$ & $3.9 \mathrm{E}-04$ & $\mathrm{Tl}_{2} \mathrm{O}$ & $1.9 \mathrm{E}-05$ & & \\
\hline${ }^{239} \mathrm{PuO}_{2}$ & 4.6E-07 & $\mathrm{V}_{2} \mathrm{O}_{5}$ & $2.8 \mathrm{E}-05$ & & \\
\hline${ }^{240} \mathrm{PuO}_{2}$ & 2.2E-08 & $\mathrm{WO}_{3}$ & 2.2E-05 & & \\
\hline${ }^{241} \mathrm{Am}_{2} \mathrm{O}_{3}$ & $1.0 \mathrm{E}-07$ & $\mathrm{Y}_{2} \mathrm{O}_{3}$ & $9.0 \mathrm{E}-06$ & & \\
\hline${ }^{241} \mathrm{PuO}_{2}$ & $2.8 \mathrm{E}-10$ & $\mathrm{ZnO}$ & $1.6 \mathrm{E}-05$ & & \\
\hline${ }^{242} \mathrm{Cm}_{2} \mathrm{O}_{3}$ & $2.1 \mathrm{E}-13$ & & & & \\
\hline${ }^{242} \mathrm{PuO}_{2}$ & $1.1 \mathrm{E}-10$ & & & & \\
\hline${ }^{243} \mathrm{Am}_{2} \mathrm{O}_{3}$ & $9.4 \mathrm{E}-11$ & & & & \\
\hline${ }^{243} \mathrm{Cm}_{2} \mathrm{O}_{3}$ & $9.7 \mathrm{E}-13$ & & & & \\
\hline${ }^{244} \mathrm{Cm}_{2} \mathrm{O}_{3}$ & $4.4 \mathrm{E}-12$ & & & & \\
\hline${ }^{59} \mathrm{NiO}$ & $2.8 \mathrm{E}-08$ & & & & \\
\hline${ }^{60} \mathrm{CoO}$ & $1.6 \mathrm{E}-11$ & & & & \\
\hline${ }^{63} \mathrm{NiO}$ & $3.6 \mathrm{E}-09$ & & & & \\
\hline${ }^{79} \mathrm{SeO}_{2}$ & $6.0 \mathrm{E}-07$ & & & & \\
\hline${ }^{90} \mathrm{SrO}$ & $3.7 \mathrm{E}-07$ & & & & \\
\hline${ }^{90} \mathrm{Y}_{2} \mathrm{O}_{3}$ & 3.8E-09 & & & & \\
\hline${ }^{93} \mathrm{ZrO}_{2}$ & $9.0 \mathrm{E}-06$ & & & & \\
\hline${ }^{93} \mathrm{Nb}_{2} \mathrm{O}_{5}$ & $1.0 \mathrm{E}-10$ & & & & \\
\hline${ }^{99} \mathrm{Tc}_{2} \mathrm{O}_{7}$ & 4.3E-05 & & & & \\
\hline
\end{tabular}


Table 5.16. Cluster F Waste Oxide Composition

\begin{tabular}{|c|c|c|c|c|c|}
\hline $\begin{array}{c}\text { Radioactive } \\
\text { oxides }\end{array}$ & Wt. frac. & $\begin{array}{l}\text { Supplemental } \\
\text { Species Oxides }\end{array}$ & Wt. frac. & $\begin{array}{c}\text { BBI Species } \\
\text { Oxides }\end{array}$ & Wt. frac. \\
\hline${ }^{106} \mathrm{RuO}_{2}$ & $0.0 \mathrm{E}+00$ & $\mathrm{Ag}_{2} \mathrm{O}$ & $7.9 \mathrm{E}-06$ & $\mathrm{Al}_{2} \mathrm{O}_{3}$ & 0.084 \\
\hline${ }^{113} \mathrm{CdO}$ & $1.5 \mathrm{E}-10$ & $\mathrm{As}_{2} \mathrm{O}_{5}$ & 8.7E-06 & $\mathrm{Bi}_{2} \mathrm{O}_{3}$ & 9.5E-05 \\
\hline${ }^{12} 5 \mathrm{Sb}_{2} \mathrm{O}_{3}$ & $2.4 \mathrm{E}-12$ & $\mathrm{~B}_{2} \mathrm{O}_{3}$ & 8.9E-05 & $\mathrm{CaO}$ & 0.00036 \\
\hline${ }^{126} \mathrm{SnO}_{2}$ & 2.7E-07 & $\mathrm{BaO}$ & 1.3E-05 & $\mathrm{Cl}$ & 0.010 \\
\hline${ }^{129} \mathrm{I}$ & $4.9 \mathrm{E}-06$ & $\mathrm{BeO}$ & $2.9 \mathrm{E}-06$ & $\mathrm{Cr}_{2} \mathrm{O}_{3}$ & 0.0062 \\
\hline${ }^{134} \mathrm{Cs}_{2} \mathrm{O}$ & $2.0 \mathrm{E}-17$ & $\mathrm{CdO}$ & $1.4 \mathrm{E}-05$ & $\mathrm{~F}$ & 0.0427 \\
\hline${ }^{137} \mathrm{Cs}_{2} \mathrm{O}$ & 4.6E-10 & $\mathrm{Ce}_{2} \mathrm{O}_{3}$ & $6.9 \mathrm{E}-04$ & $\mathrm{Fe}_{2} \mathrm{O}_{3}$ & 0.00046 \\
\hline${ }^{137} \mathrm{BaO}$ & $3.9 \mathrm{E}-13$ & $\mathrm{Co}_{2} \mathrm{O}_{3}$ & 7.3E-06 & $\mathrm{K}_{2} \mathrm{O}$ & 0.017 \\
\hline${ }^{151} \mathrm{Sm}_{2} \mathrm{O}_{3}$ & $3.6 \mathrm{E}-07$ & $\mathrm{Cs}_{2} \mathrm{O}$ & $2.0 \mathrm{E}-09$ & $\mathrm{La}_{2} \mathrm{O}_{3}$ & $1.1 \mathrm{E}-05$ \\
\hline${ }^{152} \mathrm{Eu}_{2} \mathrm{O}_{3}$ & 2.7E-11 & $\mathrm{CuO}$ & $3.9 \mathrm{E}-06$ & $\mathrm{MnO}$ & $1.6 \mathrm{E}-05$ \\
\hline${ }^{154} \mathrm{Eu}_{2} \mathrm{O}_{3}$ & $1.5 \mathrm{E}-10$ & $\mathrm{Li}_{2} \mathrm{O}$ & $2.3 \mathrm{E}-06$ & $\mathrm{Na}_{2} \mathrm{O}$ & 0.774 \\
\hline${ }^{15} 5 \mathrm{Eu}_{2} \mathrm{O}_{3}$ & $2.4 \mathrm{E}-11$ & $\mathrm{MgO}$ & $4.4 \mathrm{E}-05$ & $\mathrm{NiO}$ & $1.4 \mathrm{E}-04$ \\
\hline${ }^{226} \mathrm{RaO}$ & $1.4 \mathrm{E}-08$ & $\mathrm{MoO}_{3}$ & $1.0 \mathrm{E}-04$ & $\mathrm{PbO}$ & 0.00012 \\
\hline${ }^{227} \mathrm{Ac}_{2} \mathrm{O}_{3}$ & $3.6 \mathrm{E}-14$ & $\mathrm{Nd}_{2} \mathrm{O}_{3}$ & $9.1 \mathrm{E}-06$ & $\mathrm{P}_{2} \mathrm{O}_{5}$ & 0.0323 \\
\hline${ }^{228} \mathrm{RaO}$ & $5.4 \mathrm{E}-13$ & $\mathrm{PdO}$ & $2.5 \mathrm{E}-07$ & $\mathrm{SiO}_{2}$ & 0.0084 \\
\hline${ }^{229} \mathrm{ThO}_{2}$ & 3.3E-11 & $\mathrm{Pr}_{2} \mathrm{O}_{3}$ & 5.8E-08 & $\mathrm{SO}_{3}$ & 0.0195 \\
\hline${ }^{231} \mathrm{~Pa}_{2} \mathrm{O}_{5}$ & $1.4 \mathrm{E}-10$ & $\mathrm{Rb}_{2} \mathrm{O}$ & $3.8 \mathrm{E}-07$ & $\mathrm{SrO}$ & $3.4 \mathrm{E}-05$ \\
\hline${ }^{232} \mathrm{ThO}_{2}$ & $2.4 \mathrm{E}-04$ & $\mathrm{Rh}_{2} \mathrm{O}_{3}$ & $4.2 \mathrm{E}-07$ & $\mathrm{ZrO}_{2}$ & 0.0024 \\
\hline${ }^{232} \mathrm{UO}_{3}$ & 3.0E-13 & $\mathrm{RuO}_{2}$ & $2.2 \mathrm{E}-06$ & \multirow{26}{*}{\multicolumn{2}{|c|}{$\begin{array}{l}\text { Note that the oxide weight } \\
\text { fractions reported above } \\
\text { and immediately to the left } \\
\text { are for stable isotopes } \\
\text { alone. }\end{array}$}} \\
\hline${ }^{233} \mathrm{UO}_{3}$ & $5.4 \mathrm{E}-09$ & $\mathrm{Sb}_{2} \mathrm{O}_{3}$ & $7.0 \mathrm{E}-06$ & & \\
\hline${ }^{234} \mathrm{UO}_{3}$ & $2.5 \mathrm{E}-08$ & $\mathrm{SeO}_{2}$ & $1.1 \mathrm{E}-04$ & & \\
\hline${ }^{235} \mathrm{UO}_{3}$ & $3.8 \mathrm{E}-06$ & $\mathrm{Ta}_{2} \mathrm{O}_{5}$ & $7.1 \mathrm{E}-08$ & & \\
\hline${ }^{236} \mathrm{UO}_{3}$ & $1.5 \mathrm{E}-07$ & $\mathrm{TeO}_{2}$ & $8.0 \mathrm{E}-08$ & & \\
\hline${ }^{237} \mathrm{NpO}_{2}$ & $2.2 \mathrm{E}-06$ & $\mathrm{ThO}_{2}$ & $1.1 \mathrm{E}-06$ & & \\
\hline${ }^{238} \mathrm{PuO}_{2}$ & $6.2 \mathrm{E}-11$ & $\mathrm{TiO}_{2}$ & $1.3 \mathrm{E}-06$ & & \\
\hline${ }^{238} \mathrm{UO}_{3}$ & $5.5 \mathrm{E}-04$ & $\mathrm{Tl}_{2} \mathrm{O}$ & $1.2 \mathrm{E}-05$ & & \\
\hline${ }^{239} \mathrm{PuO}_{2}$ & $3.2 \mathrm{E}-07$ & $\mathrm{~V}_{2} \mathrm{O}_{5}$ & $6.8 \mathrm{E}-06$ & & \\
\hline${ }^{240} \mathrm{PuO}_{2}$ & $1.7 \mathrm{E}-08$ & $\mathrm{WO}_{3}$ & $1.9 \mathrm{E}-05$ & & \\
\hline${ }^{241} \mathrm{Am}_{2} \mathrm{O}_{3}$ & $3.5 \mathrm{E}-08$ & $\mathrm{Y}_{2} \mathrm{O}_{3}$ & $3.0 \mathrm{E}-07$ & & \\
\hline${ }^{241} \mathrm{PuO}_{2}$ & $4.1 \mathrm{E}-10$ & $\mathrm{ZnO}$ & $5.4 \mathrm{E}-06$ & & \\
\hline${ }^{242} \mathrm{Cm}_{2} \mathrm{O}_{3}$ & $3.2 \mathrm{E}-14$ & & & & \\
\hline${ }^{242} \mathrm{PuO}_{2}$ & $1.5 \mathrm{E}-10$ & & & & \\
\hline${ }^{243} \mathrm{Am}_{2} \mathrm{O}_{3}$ & 3.2E-11 & & & & \\
\hline${ }^{243} \mathrm{Cm}_{2} \mathrm{O}_{3}$ & $1.5 \mathrm{E}-13$ & & & & \\
\hline${ }^{244} \mathrm{Cm}_{2} \mathrm{O}_{3}$ & $1.2 \mathrm{E}-12$ & & & & \\
\hline${ }^{59} \mathrm{NiO}$ & $1.8 \mathrm{E}-08$ & & & & \\
\hline${ }^{60} \mathrm{CoO}$ & $2.9 \mathrm{E}-12$ & & & & \\
\hline${ }^{63} \mathrm{NiO}$ & $1.3 \mathrm{E}-09$ & & & & \\
\hline${ }^{79} \mathrm{SeO}_{2}$ & $5.0 \mathrm{E}-07$ & & & & \\
\hline${ }^{90} \mathrm{SrO}$ & $8.6 \mathrm{E}-07$ & & & & \\
\hline${ }^{90} \mathrm{Y}_{2} \mathrm{O}_{3}$ & $8.6 \mathrm{E}-10$ & & & & \\
\hline${ }^{93} \mathrm{ZrO}_{2}$ & $1.2 \mathrm{E}-05$ & & & & \\
\hline${ }^{93} \mathrm{Nb}_{2} \mathrm{O}_{5}$ & $1.1 \mathrm{E}-10$ & & & & \\
\hline${ }^{99} \mathrm{Tc}_{2} \mathrm{O}_{7}$ & $2.9 \mathrm{E}-05$ & & & & \\
\hline
\end{tabular}


Table 5.17. Cluster O Waste Oxide Composition

\begin{tabular}{|c|c|c|c|c|c|}
\hline $\begin{array}{c}\text { Radioactive } \\
\text { oxides }\end{array}$ & Wt. frac. & $\begin{array}{l}\text { Supplemental } \\
\text { Species Oxides }\end{array}$ & Wt. frac. & $\begin{array}{c}\text { BBI Species } \\
\text { Oxides }\end{array}$ & Wt. frac. \\
\hline${ }^{106} \mathrm{RuO}_{2}$ & $0.0 \mathrm{E}+00$ & $\mathrm{Ag}_{2} \mathrm{O}$ & $4.4 \mathrm{E}-06$ & $\mathrm{Al}_{2} \mathrm{O}_{3}$ & 0.016 \\
\hline${ }^{113} \mathrm{CdO}$ & 5.3E-12 & $\mathrm{As}_{2} \mathrm{O}_{5}$ & 2.7E-06 & $\mathrm{Bi}_{2} \mathrm{O}_{3}$ & $1.1 \mathrm{E}-05$ \\
\hline${ }^{12} 5 \mathrm{Sb}_{2} \mathrm{O}_{3}$ & $5.8 \mathrm{E}-14$ & $\mathrm{~B}_{2} \mathrm{O}_{3}$ & $1.5 \mathrm{E}-05$ & $\mathrm{CaO}$ & 0.00016 \\
\hline${ }^{126} \mathrm{SnO}_{2}$ & $6.5 \mathrm{E}-09$ & $\mathrm{BaO}$ & $6.8 \mathrm{E}-07$ & $\mathrm{Cl}$ & 0.085 \\
\hline${ }^{129} \mathrm{I}$ & 1.4E-04 & $\mathrm{BeO}$ & $3.9 \mathrm{E}-07$ & $\mathrm{Cr}_{2} \mathrm{O}_{3}$ & 0.0045 \\
\hline${ }^{134} \mathrm{Cs}_{2} \mathrm{O}$ & $0.0 \mathrm{E}+00$ & $\mathrm{CdO}$ & $1.5 \mathrm{E}-05$ & $F$ & 0.0575 \\
\hline${ }^{137} \mathrm{Cs}_{2} \mathrm{O}$ & $4.9 \mathrm{E}-10$ & $\mathrm{Ce}_{2} \mathrm{O}_{3}$ & $1.4 \mathrm{E}-04$ & $\mathrm{Fe}_{2} \mathrm{O}_{3}$ & 0.00021 \\
\hline${ }^{137} \mathrm{BaO}$ & $8.2 \mathrm{E}-14$ & $\mathrm{Co}_{2} \mathrm{O}_{3}$ & $4.2 \mathrm{E}-07$ & $\mathrm{~K}_{2} \mathrm{O}$ & 0.003 \\
\hline${ }^{151} \mathrm{Sm}_{2} \mathrm{O}_{3}$ & $4.7 \mathrm{E}-09$ & $\mathrm{Cs}_{2} \mathrm{O}$ & $2.5 \mathrm{E}-09$ & $\mathrm{La}_{2} \mathrm{O}_{3}$ & 2.6E-06 \\
\hline${ }^{152} \mathrm{Eu}_{2} \mathrm{O}_{3}$ & $2.0 \mathrm{E}-13$ & $\mathrm{CuO}$ & $1.7 \mathrm{E}-07$ & $\mathrm{MnO}$ & $1.3 \mathrm{E}-05$ \\
\hline${ }^{154} \mathrm{Eu}_{2} \mathrm{O}_{3}$ & $3.5 \mathrm{E}-12$ & $\mathrm{Li}_{2} \mathrm{O}$ & $2.6 \mathrm{E}-07$ & $\mathrm{Na}_{2} \mathrm{O}$ & 0.508 \\
\hline${ }^{15} 5 \mathrm{Eu}_{2} \mathrm{O}_{3}$ & $1.9 \mathrm{E}-13$ & $\mathrm{MgO}$ & $8.1 \mathrm{E}-07$ & $\mathrm{NiO}$ & $1.5 \mathrm{E}-04$ \\
\hline${ }^{226} \mathrm{RaO}$ & $3.5 \mathrm{E}-07$ & $\mathrm{MoO}_{3}$ & 5.6E-05 & $\mathrm{PbO}$ & 0.00005 \\
\hline${ }^{227} \mathrm{Ac}_{2} \mathrm{O}_{3}$ & $3.8 \mathrm{E}-16$ & $\mathrm{Nd}_{2} \mathrm{O}_{3}$ & 3.4E-06 & $\mathrm{P}_{2} \mathrm{O}_{5}$ & 0.0057 \\
\hline${ }^{228} \mathrm{RaO}$ & $1.9 \mathrm{E}-11$ & PdO & $8.6 \mathrm{E}-09$ & $\mathrm{SiO}_{2}$ & 0.0008 \\
\hline${ }^{229} \mathrm{ThO}_{2}$ & $3.2 \mathrm{E}-12$ & $\mathrm{Pr}_{2} \mathrm{O}_{3}$ & $3.8 \mathrm{E}-08$ & $\mathrm{SO}_{3}$ & 0.3099 \\
\hline${ }^{231} \mathrm{~Pa}_{2} \mathrm{O}_{5}$ & $2.0 \mathrm{E}-12$ & $\mathrm{Rb}_{2} \mathrm{O}$ & $2.3 \mathrm{E}-07$ & $\mathrm{SrO}$ & 5.7E-05 \\
\hline${ }^{232} \mathrm{ThO}_{2}$ & $1.5 \mathrm{E}-05$ & $\mathrm{Rh}_{2} \mathrm{O}_{3}$ & $4.4 \mathrm{E}-07$ & $\mathrm{ZrO}_{2}$ & $2.1 \mathrm{E}-06$ \\
\hline${ }^{232} \mathrm{UO}_{3}$ & 6.6E-14 & $\mathrm{RuO}_{2}$ & 4.4E-05 & \multirow{26}{*}{\multicolumn{2}{|c|}{$\begin{array}{l}\text { Note that the oxide weight } \\
\text { fractions reported above } \\
\text { and immediately to the left } \\
\text { are for stable isotopes } \\
\text { alone. }\end{array}$}} \\
\hline${ }^{233} \mathrm{UO}_{3}$ & $8.0 \mathrm{E}-10$ & $\mathrm{Sb}_{2} \mathrm{O}_{3}$ & $2.3 \mathrm{E}-06$ & & \\
\hline${ }^{234} \mathrm{UO}_{3}$ & 3.3E-09 & $\mathrm{SeO}_{2}$ & 7.5E-03 & & \\
\hline${ }^{235} \mathrm{UO}_{3}$ & $4.5 \mathrm{E}-07$ & $\mathrm{Ta}_{2} \mathrm{O}_{5}$ & $6.8 \mathrm{E}-09$ & & \\
\hline${ }^{236} \mathrm{UO}_{3}$ & 5.5E-09 & $\mathrm{TeO}_{2}$ & $8.8 \mathrm{E}-07$ & & \\
\hline${ }^{237} \mathrm{NpO}_{2}$ & 5.2E-08 & $\mathrm{ThO}_{2}$ & $3.4 \mathrm{E}-09$ & & \\
\hline${ }^{238} \mathrm{PuO}_{2}$ & $1.8 \mathrm{E}-12$ & $\mathrm{TiO}_{2}$ & $1.4 \mathrm{E}-07$ & & \\
\hline${ }^{238} \mathrm{UO}_{3}$ & 6.4E-05 & $\mathrm{Tl}_{2} \mathrm{O}$ & $1.4 \mathrm{E}-04$ & & \\
\hline${ }^{239} \mathrm{PuO}_{2}$ & $1.0 \mathrm{E}-08$ & $\mathrm{~V}_{2} \mathrm{O}_{5}$ & $2.3 \mathrm{E}-06$ & & \\
\hline${ }^{240} \mathrm{PuO}_{2}$ & $5.1 \mathrm{E}-10$ & $\mathrm{WO}_{3}$ & $3.6 \mathrm{E}-06$ & & \\
\hline${ }^{241} \mathrm{Am}_{2} \mathrm{O}_{3}$ & 3.4E-09 & $\mathrm{Y}_{2} \mathrm{O}_{3}$ & 5.5E-07 & & \\
\hline${ }^{241} \mathrm{PuO}_{2}$ & $3.3 \mathrm{E}-12$ & $\mathrm{ZnO}$ & $1.8 \mathrm{E}-07$ & & \\
\hline${ }^{242} \mathrm{Cm}_{2} \mathrm{O}_{3}$ & 7.4E-15 & & & & \\
\hline${ }^{242} \mathrm{PuO}_{2}$ & $1.8 \mathrm{E}-12$ & & & & \\
\hline${ }^{243} \mathrm{Am}_{2} \mathrm{O}_{3}$ & 2.2E-12 & & & & \\
\hline${ }^{243} \mathrm{Cm}_{2} \mathrm{O}_{3}$ & $1.1 \mathrm{E}-14$ & & & & \\
\hline${ }^{244} \mathrm{Cm}_{2} \mathrm{O}_{3}$ & 4.0E-14 & & & & \\
\hline${ }^{59} \mathrm{NiO}$ & 2.8E-09 & & & & \\
\hline${ }^{60} \mathrm{CoO}$ & $4.8 \mathrm{E}-14$ & & & & \\
\hline${ }^{63} \mathrm{NiO}$ & $3.3 \mathrm{E}-10$ & & & & \\
\hline${ }^{79} \mathrm{SeO}_{2}$ & $1.2 \mathrm{E}-08$ & & & & \\
\hline${ }^{90} \mathrm{SrO}$ & $1.4 \mathrm{E}-07$ & & & & \\
\hline${ }^{90} \mathrm{Y}_{2} \mathrm{O}_{3}$ & $6.5 \mathrm{E}-08$ & & & & \\
\hline${ }^{93} \mathrm{ZrO}_{2}$ & $1.3 \mathrm{E}-07$ & & & & \\
\hline${ }^{93} \mathrm{Nb}_{2} \mathrm{O}_{5}$ & $2.2 \mathrm{E}-12$ & & & & \\
\hline${ }^{99} \mathrm{Tc}_{2} \mathrm{O}_{7}$ & $4.4 \mathrm{E}-04$ & & & & \\
\hline
\end{tabular}


Table 5.18. Cluster Y1 Waste Oxide Composition

\begin{tabular}{|c|c|c|c|c|c|}
\hline $\begin{array}{c}\text { Radioactive } \\
\text { oxides }\end{array}$ & Wt. frac. & $\begin{array}{c}\text { Supplemental } \\
\text { Species Oxides }\end{array}$ & Wt. frac. & $\begin{array}{c}\text { BBI Species } \\
\text { Oxides }\end{array}$ & Wt. frac. \\
\hline${ }^{106} \mathrm{RuO}_{2}$ & $6.0 \mathrm{E}-27$ & $\mathrm{Ag}_{2} \mathrm{O}$ & $0.0 \mathrm{E}+00$ & $\mathrm{Al}_{2} \mathrm{O}_{3}$ & 0.002 \\
\hline${ }^{113} \mathrm{CdO}$ & $2.0 \mathrm{E}-13$ & $\mathrm{As}_{2} \mathrm{O}_{5}$ & $0.0 \mathrm{E}+00$ & $\mathrm{Bi}_{2} \mathrm{O}_{3}$ & 3.8E-06 \\
\hline${ }^{12} 5 \mathrm{Sb}_{2} \mathrm{O}_{3}$ & $1.0 \mathrm{E}-15$ & $\mathrm{~B}_{2} \mathrm{O}_{3}$ & $0.0 \mathrm{E}+00$ & $\mathrm{CaO}$ & 0.00013 \\
\hline${ }^{126} \mathrm{SnO}_{2}$ & $1.1 \mathrm{E}-10$ & $\mathrm{BaO}$ & $0.0 \mathrm{E}+00$ & $\mathrm{Cl}$ & 0.001 \\
\hline${ }^{129} \mathrm{I}$ & 7.6E-09 & $\mathrm{BeO}$ & $0.0 \mathrm{E}+00$ & $\mathrm{Cr}_{2} \mathrm{O}_{3}$ & 0.0001 \\
\hline${ }^{134} \mathrm{Cs}_{2} \mathrm{O}$ & 6.9E-18 & $\mathrm{CdO}$ & $0.0 \mathrm{E}+00$ & $\mathrm{~F}$ & 0.0395 \\
\hline${ }^{137} \mathrm{Cs}_{2} \mathrm{O}$ & $3.1 \mathrm{E}-08$ & $\mathrm{Ce}_{2} \mathrm{O}_{3}$ & $0.0 \mathrm{E}+00$ & $\mathrm{Fe}_{2} \mathrm{O}_{3}$ & 0.00044 \\
\hline${ }^{137} \mathrm{BaO}$ & $5.0 \mathrm{E}-15$ & $\mathrm{Co}_{2} \mathrm{O}_{3}$ & $0.0 \mathrm{E}+00$ & $\mathrm{~K}_{2} \mathrm{O}$ & 0.001 \\
\hline${ }^{151} \mathrm{Sm}_{2} \mathrm{O}_{3}$ & $6.6 \mathrm{E}-13$ & $\mathrm{Cs}_{2} \mathrm{O}$ & $0.0 \mathrm{E}+00$ & $\mathrm{La}_{2} \mathrm{O}_{3}$ & $2.5 \mathrm{E}-07$ \\
\hline${ }^{152} \mathrm{Eu}_{2} \mathrm{O}_{3}$ & $1.2 \mathrm{E}-15$ & $\mathrm{CuO}$ & $0.0 \mathrm{E}+00$ & $\mathrm{MnO}$ & $2.8 \mathrm{E}-05$ \\
\hline${ }^{154} \mathrm{Eu}_{2} \mathrm{O}_{3}$ & 5.1E-14 & $\mathrm{Li}_{2} \mathrm{O}$ & $0.0 \mathrm{E}+00$ & $\mathrm{Na}_{2} \mathrm{O}$ & 0.531 \\
\hline${ }^{15} 5 \mathrm{Eu}_{2} \mathrm{O}_{3}$ & $1.4 \mathrm{E}-14$ & $\mathrm{MgO}$ & $0.0 \mathrm{E}+00$ & $\mathrm{NiO}$ & 8.6E-06 \\
\hline${ }^{226} \mathrm{RaO}$ & $4.2 \mathrm{E}-15$ & $\mathrm{MoO}_{3}$ & $0.0 \mathrm{E}+00$ & $\mathrm{PbO}$ & 0.00004 \\
\hline${ }^{227} \mathrm{Ac}_{2} \mathrm{O}_{3}$ & $5.8 \mathrm{E}-19$ & $\mathrm{Nd}_{2} \mathrm{O}_{3}$ & $0.0 \mathrm{E}+00$ & $\mathrm{P}_{2} \mathrm{O}_{5}$ & 0.4191 \\
\hline${ }^{228} \mathrm{RaO}$ & $9.3 \mathrm{E}-23$ & $\mathrm{PdO}$ & $0.0 \mathrm{E}+00$ & $\mathrm{SiO}_{2}$ & 0.0008 \\
\hline${ }^{229} \mathrm{ThO}_{2}$ & $3.4 \mathrm{E}-18$ & $\mathrm{Pr}_{2} \mathrm{O}_{3}$ & $0.0 \mathrm{E}+00$ & $\mathrm{SO}_{3}$ & 0.0057 \\
\hline${ }^{231} \mathrm{~Pa}_{2} \mathrm{O}_{5}$ & 7.4E-15 & $\mathrm{Rb}_{2} \mathrm{O}$ & $0.0 \mathrm{E}+00$ & $\mathrm{SrO}$ & $0.0 \mathrm{E}+00$ \\
\hline${ }^{232} \mathrm{ThO}_{2}$ & $5.5 \mathrm{E}-14$ & $\mathrm{Rh}_{2} \mathrm{O}_{3}$ & $0.0 \mathrm{E}+00$ & $\mathrm{ZrO}_{2}$ & 2.6E-06 \\
\hline${ }^{232} \mathrm{UO}_{3}$ & $8.1 \mathrm{E}-18$ & $\mathrm{RuO}_{2}$ & $0.0 \mathrm{E}+00$ & \multirow{26}{*}{\multicolumn{2}{|c|}{$\begin{array}{l}\text { Note that the oxide weight } \\
\text { fractions reported above } \\
\text { and immediately to the left } \\
\text { are for the sum of stable } \\
\text { plus radioactive isotopes. }\end{array}$}} \\
\hline${ }^{233} \mathrm{UO}_{3}$ & $1.5 \mathrm{E}-15$ & $\mathrm{Sb}_{2} \mathrm{O}_{3}$ & $0.0 \mathrm{E}+00$ & & \\
\hline${ }^{234} \mathrm{UO}_{3}$ & 2.1E-09 & $\mathrm{SeO}_{2}$ & $0.0 \mathrm{E}+00$ & & \\
\hline${ }^{235} \mathrm{UO}_{3}$ & $2.8 \mathrm{E}-07$ & $\mathrm{Ta}_{2} \mathrm{O}_{5}$ & $0.0 \mathrm{E}+00$ & & \\
\hline${ }^{236} \mathrm{UO}_{3}$ & 2.4E-09 & $\mathrm{TeO}_{2}$ & $0.0 \mathrm{E}+00$ & & \\
\hline${ }^{237} \mathrm{NpO}_{2}$ & $3.1 \mathrm{E}-09$ & $\mathrm{ThO}_{2}$ & 5.5E-14 & & \\
\hline${ }^{238} \mathrm{PuO}_{2}$ & $2.4 \mathrm{E}-13$ & $\mathrm{TiO}_{2}$ & $0.0 \mathrm{E}+00$ & & \\
\hline${ }^{238} \mathrm{UO}_{3}$ & $4.0 \mathrm{E}-05$ & $\mathrm{Tl}_{2} \mathrm{O}$ & $0.0 \mathrm{E}+00$ & & \\
\hline${ }^{239} \mathrm{PuO}_{2}$ & $9.0 \mathrm{E}-09$ & $\mathrm{~V}_{2} \mathrm{O}_{5}$ & $0.0 \mathrm{E}+00$ & & \\
\hline${ }^{240} \mathrm{PuO}_{2}$ & $2.8 \mathrm{E}-10$ & $\mathrm{WO}_{3}$ & $0.0 \mathrm{E}+00$ & & \\
\hline${ }^{241} \mathrm{Am}_{2} \mathrm{O}_{3}$ & $1.1 \mathrm{E}-11$ & $\mathrm{Y}_{2} \mathrm{O}_{3}$ & $0.0 \mathrm{E}+00$ & & \\
\hline${ }^{241} \mathrm{PuO}_{2}$ & $1.1 \mathrm{E}-12$ & $\mathrm{ZnO}$ & $0.0 \mathrm{E}+00$ & & \\
\hline${ }^{242} \mathrm{Cm}_{2} \mathrm{O}_{3}$ & $7.0 \mathrm{E}-18$ & & & & \\
\hline${ }^{242} \mathrm{PuO}_{2}$ & $2.4 \mathrm{E}-13$ & & & & \\
\hline${ }^{243} \mathrm{Am}_{2} \mathrm{O}_{3}$ & 2.4E-14 & & & & \\
\hline${ }^{243} \mathrm{Cm}_{2} \mathrm{O}_{3}$ & $5.0 \mathrm{E}-18$ & & & & \\
\hline${ }^{244} \mathrm{Cm}_{2} \mathrm{O}_{3}$ & 7.1E-17 & & & & \\
\hline${ }^{59} \mathrm{NiO}$ & $7.0 \mathrm{E}-10$ & & & & \\
\hline${ }^{60} \mathrm{CoO}$ & $1.5 \mathrm{E}-14$ & & & & \\
\hline${ }^{63} \mathrm{NiO}$ & $9.1 \mathrm{E}-11$ & & & & \\
\hline${ }^{79} \mathrm{SeO}_{2}$ & 8.5E-11 & & & & \\
\hline${ }^{90} \mathrm{SrO}$ & $0.0 \mathrm{E}+00$ & & & & \\
\hline${ }^{90} \mathrm{Y}_{2} \mathrm{O}_{3}$ & $5.1 \mathrm{E}-13$ & & & & \\
\hline${ }^{93} \mathrm{ZrO}_{2}$ & 2.9E-08 & & & & \\
\hline${ }^{93} \mathrm{Nb}_{2} \mathrm{O}_{5}$ & $6.1 \mathrm{E}-13$ & & & & \\
\hline${ }^{99} \mathrm{Tc}_{2} \mathrm{O}_{7}$ & $9.2 \mathrm{E}-08$ & & & & \\
\hline
\end{tabular}


Table 5.19. Cluster Y2 Waste Oxide Composition

\begin{tabular}{|c|c|c|c|c|c|}
\hline $\begin{array}{c}\text { Radioactive } \\
\text { oxides }\end{array}$ & $\begin{array}{l}\text { Wt. } \\
\text { frac. }\end{array}$ & $\begin{array}{c}\text { Supplemental } \\
\text { Species Oxides }\end{array}$ & $\begin{array}{l}\text { Wt. } \\
\text { frac. }\end{array}$ & $\begin{array}{c}\text { BBI Species } \\
\text { Oxides }\end{array}$ & Wt. frac. \\
\hline${ }^{106} \mathrm{RuO}_{2}$ & $0.0 \mathrm{E}+00$ & $\mathrm{Ag}_{2} \mathrm{O}$ & $0.0 \mathrm{E}+00$ & $\mathrm{Al}_{2} \mathrm{O}_{3}$ & 0.263 \\
\hline${ }^{113} \mathrm{CdO}$ & $2.4 \mathrm{E}-12$ & $\mathrm{As}_{2} \mathrm{O}_{5}$ & $0.0 \mathrm{E}+00$ & $\mathrm{Bi}_{2} \mathrm{O}_{3}$ & $0.0 \mathrm{E}+00$ \\
\hline${ }^{12} 5 \mathrm{Sb}_{2} \mathrm{O}_{3}$ & $0.0 \mathrm{E}+00$ & $\mathrm{~B}_{2} \mathrm{O}_{3}$ & $0.0 \mathrm{E}+00$ & $\mathrm{CaO}$ & 0.00015 \\
\hline${ }^{126} \mathrm{SnO}_{2}$ & $1.1 \mathrm{E}-11$ & $\mathrm{BaO}$ & $0.0 \mathrm{E}+00$ & $\mathrm{Cl}$ & 0.003 \\
\hline${ }^{129} \mathrm{I}$ & $1.9 \mathrm{E}-06$ & $\mathrm{BeO}$ & $0.0 \mathrm{E}+00$ & $\mathrm{Cr}_{2} \mathrm{O}_{3}$ & 0.0015 \\
\hline${ }^{134} \mathrm{Cs}_{2} \mathrm{O}$ & $1.1 \mathrm{E}-15$ & $\mathrm{CdO}$ & $0.0 \mathrm{E}+00$ & $\mathrm{~F}$ & 0.0056 \\
\hline${ }^{137} \mathrm{Cs}_{2} \mathrm{O}$ & $2.4 \mathrm{E}-07$ & $\mathrm{Ce}_{2} \mathrm{O}_{3}$ & $0.0 \mathrm{E}+00$ & $\mathrm{Fe}_{2} \mathrm{O}_{3}$ & 0.00000 \\
\hline${ }^{137} \mathrm{BaO}$ & $3.9 \mathrm{E}-14$ & $\mathrm{Co}_{2} \mathrm{O}_{3}$ & $0.0 \mathrm{E}+00$ & $\mathrm{~K}_{2} \mathrm{O}$ & 0.001 \\
\hline${ }^{151} \mathrm{Sm}_{2} \mathrm{O}_{3}$ & $7.0 \mathrm{E}-13$ & $\mathrm{Cs}_{2} \mathrm{O}$ & $0.0 \mathrm{E}+00$ & $\mathrm{La}_{2} \mathrm{O}_{3}$ & 1.8E-06 \\
\hline${ }^{152} \mathrm{Eu}_{2} \mathrm{O}_{3}$ & $1.6 \mathrm{E}-15$ & $\mathrm{CuO}$ & $0.0 \mathrm{E}+00$ & $\mathrm{MnO}$ & $0.0 \mathrm{E}+00$ \\
\hline${ }^{154} \mathrm{Eu}_{2} \mathrm{O}_{3}$ & $7.0 \mathrm{E}-14$ & $\mathrm{Li}_{2} \mathrm{O}$ & $0.0 \mathrm{E}+00$ & $\mathrm{Na}_{2} \mathrm{O}$ & 0.511 \\
\hline${ }^{15} 5 \mathrm{Eu}_{2} \mathrm{O}_{3}$ & $1.9 \mathrm{E}-14$ & $\mathrm{MgO}$ & $0.0 \mathrm{E}+00$ & $\mathrm{NiO}$ & $0.0 \mathrm{E}+00$ \\
\hline${ }^{226} \mathrm{RaO}$ & 5.7E-16 & $\mathrm{MoO}_{3}$ & $0.0 \mathrm{E}+00$ & $\mathrm{PbO}$ & 0.00012 \\
\hline${ }^{227} \mathrm{Ac}_{2} \mathrm{O}_{3}$ & $3.5 \mathrm{E}-16$ & $\mathrm{Nd}_{2} \mathrm{O}_{3}$ & $0.0 \mathrm{E}+00$ & $\mathrm{P}_{2} \mathrm{O}_{5}$ & 0.1294 \\
\hline${ }^{228} \mathrm{RaO}$ & 4.6E-14 & $\mathrm{PdO}$ & $0.0 \mathrm{E}+00$ & $\mathrm{SiO}_{2}$ & 0.0008 \\
\hline${ }^{229} \mathrm{ThO}_{2}$ & $3.8 \mathrm{E}-10$ & $\mathrm{Pr}_{2} \mathrm{O}_{3}$ & $0.0 \mathrm{E}+00$ & $\mathrm{SO}_{3}$ & 0.0627 \\
\hline${ }^{231} \mathrm{~Pa}_{2} \mathrm{O}_{5}$ & $0.0 \mathrm{E}+00$ & $\mathrm{Rb}_{2} \mathrm{O}$ & $0.0 \mathrm{E}+00$ & SrO & $0.0 \mathrm{E}+00$ \\
\hline${ }^{232} \mathrm{ThO}_{2}$ & 3.4E-03 & $\mathrm{Rh}_{2} \mathrm{O}_{3}$ & $0.0 \mathrm{E}+00$ & $\mathrm{ZrO}_{2}$ & 0 \\
\hline${ }^{232} \mathrm{UO}_{3}$ & $5.7 \mathrm{E}-12$ & $\mathrm{RuO}_{2}$ & $0.0 \mathrm{E}+00$ & \multirow{26}{*}{\multicolumn{2}{|c|}{$\begin{array}{l}\text { Note that the oxide weight } \\
\text { fractions reported above } \\
\text { and immediately to the left } \\
\text { are for the sum of stable } \\
\text { plus radioactive isotopes. }\end{array}$}} \\
\hline${ }^{233} \mathrm{UO}_{3}$ & 7.7E-07 & $\mathrm{Sb}_{2} \mathrm{O}_{3}$ & $0.0 \mathrm{E}+00$ & & \\
\hline${ }^{234} \mathrm{UO}_{3}$ & $4.0 \mathrm{E}-07$ & $\mathrm{SeO}_{2}$ & $0.0 \mathrm{E}+00$ & & \\
\hline${ }^{235} \mathrm{UO}_{3}$ & $4.8 \mathrm{E}-05$ & $\mathrm{Ta}_{2} \mathrm{O}_{5}$ & $0.0 \mathrm{E}+00$ & & \\
\hline${ }^{236} \mathrm{UO}_{3}$ & $6.5 \mathrm{E}-07$ & $\mathrm{TeO}_{2}$ & $0.0 \mathrm{E}+00$ & & \\
\hline${ }^{237} \mathrm{NpO}_{2}$ & 5.1E-09 & $\mathrm{ThO}_{2}$ & $3.4 \mathrm{E}-03$ & & \\
\hline${ }^{238} \mathrm{PuO}_{2}$ & $5.0 \mathrm{E}-10$ & $\mathrm{TiO}_{2}$ & $0.0 \mathrm{E}+00$ & & \\
\hline${ }^{238} \mathrm{UO}_{3}$ & 7.1E-03 & $\mathrm{Tl}_{2} \mathrm{O}$ & $0.0 \mathrm{E}+00$ & & \\
\hline${ }^{239} \mathrm{PuO}_{2}$ & 7.3E-06 & $\mathrm{V}_{2} \mathrm{O}_{5}$ & $0.0 \mathrm{E}+00$ & & \\
\hline${ }^{240} \mathrm{PuO}_{2}$ & $4.0 \mathrm{E}-07$ & $\mathrm{WO}_{3}$ & $0.0 \mathrm{E}+00$ & & \\
\hline${ }^{241} \mathrm{Am}_{2} \mathrm{O}_{3}$ & $1.6 \mathrm{E}-07$ & $\mathrm{Y}_{2} \mathrm{O}_{3}$ & $0.0 \mathrm{E}+00$ & & \\
\hline${ }^{241} \mathrm{PuO}_{2}$ & 4.9E-09 & $\mathrm{ZnO}$ & $0.0 \mathrm{E}+00$ & & \\
\hline${ }^{242} \mathrm{Cm}_{2} \mathrm{O}_{3}$ & $3.6 \mathrm{E}-15$ & & & & \\
\hline${ }^{242} \mathrm{PuO}_{2}$ & $1.1 \mathrm{E}-09$ & & & & \\
\hline${ }^{243} \mathrm{Am}_{2} \mathrm{O}_{3}$ & 2.9E-10 & & & & \\
\hline${ }^{243} \mathrm{Cm}_{2} \mathrm{O}_{3}$ & 3.3E-15 & & & & \\
\hline${ }^{244} \mathrm{Cm}_{2} \mathrm{O}_{3}$ & $5.1 \mathrm{E}-14$ & & & & \\
\hline${ }^{59} \mathrm{NiO}$ & $0.0 \mathrm{E}+00$ & & & & \\
\hline${ }^{60} \mathrm{CoO}$ & $0.0 \mathrm{E}+00$ & & & & \\
\hline${ }^{63} \mathrm{NiO}$ & $0.0 \mathrm{E}+00$ & & & & \\
\hline${ }^{79} \mathrm{SeO}_{2}$ & $1.1 \mathrm{E}-10$ & & & & \\
\hline${ }^{90} \mathrm{SrO}$ & $0.0 \mathrm{E}+00$ & & & & \\
\hline${ }^{90} \mathrm{Y}_{2} \mathrm{O}_{3}$ & $0.0 \mathrm{E}+00$ & & & & \\
\hline${ }^{93} \mathrm{ZrO}_{2}$ & $0.0 \mathrm{E}+00$ & & & & \\
\hline${ }^{93} \mathrm{Nb}_{2} \mathrm{O}_{5}$ & $0.0 \mathrm{E}+00$ & & & & \\
\hline${ }^{99} \mathrm{Tc}_{2} \mathrm{O}_{7}$ & $1.4 \mathrm{E}-07$ & & & & \\
\hline
\end{tabular}


Table 5.20. Cluster Y3 Waste Oxide Composition

\begin{tabular}{|c|c|c|c|c|c|}
\hline $\begin{array}{c}\text { Radioactive } \\
\text { oxides }\end{array}$ & Wt. frac. & $\begin{array}{c}\text { Supplemental Species } \\
\text { Oxides }\end{array}$ & Wt. frac. & $\begin{array}{l}\text { BBI Species } \\
\text { Oxides }\end{array}$ & Wt. frac. \\
\hline${ }^{106} \mathrm{RuO}_{2}$ & 2.7E-19 & $\mathrm{Ag}_{2} \mathrm{O}$ & $0.0 \mathrm{E}+00$ & $\mathrm{Al}_{2} \mathrm{O}_{3}$ & 0.058 \\
\hline${ }^{113} \mathrm{CdO}$ & 6.9E-11 & $\mathrm{As}_{2} \mathrm{O}_{5}$ & 5.9E-07 & $\mathrm{Bi}_{2} \mathrm{O}_{3}$ & $2.5 \mathrm{E}-04$ \\
\hline${ }^{12} 5 \mathrm{Sb}_{2} \mathrm{O}_{3}$ & $2.0 \mathrm{E}-12$ & $\mathrm{~B}_{2} \mathrm{O}_{3}$ & 3.7E-04 & $\mathrm{CaO}$ & 0.00102 \\
\hline${ }^{126} \mathrm{SnO}_{2}$ & 3.6E-09 & $\mathrm{BaO}$ & 3.3E-06 & $\mathrm{Cl}$ & 0.002 \\
\hline${ }^{129} \mathrm{I}$ & 3.8E-06 & $\mathrm{BeO}$ & 7.0E-07 & $\mathrm{Cr}_{2} \mathrm{O}_{3}$ & 0.0020 \\
\hline${ }^{134} \mathrm{Cs}_{2} \mathrm{O}$ & $1.4 \mathrm{E}-14$ & $\mathrm{CdO}$ & 4.6E-07 & $\mathrm{F}$ & 0.0386 \\
\hline${ }^{137} \mathrm{Cs}_{2} \mathrm{O}$ & 5.8E-07 & $\mathrm{Ce}_{2} \mathrm{O}_{3}$ & $0.0 \mathrm{E}+00$ & $\mathrm{Fe}_{2} \mathrm{O}_{3}$ & 0.00074 \\
\hline${ }^{137} \mathrm{BaO}$ & $9.4 \mathrm{E}-14$ & $\mathrm{Co}_{2} \mathrm{O}_{3}$ & 2.3E-05 & $\mathrm{K}_{2} \mathrm{O}$ & 0.007 \\
\hline${ }^{151} \mathrm{Sm}_{2} \mathrm{O}_{3}$ & 2.7E-11 & $\mathrm{Cs}_{2} \mathrm{O}$ & $0.0 \mathrm{E}+00$ & $\mathrm{La}_{2} \mathrm{O}_{3}$ & 2.1E-08 \\
\hline${ }^{152} \mathrm{Eu}_{2} \mathrm{O}_{3}$ & $2.7 \mathrm{E}-13$ & $\mathrm{CuO}$ & $5.8 \mathrm{E}-05$ & $\mathrm{MnO}$ & 5.0E-06 \\
\hline${ }^{154} \mathrm{Eu}_{2} \mathrm{O}_{3}$ & $1.2 \mathrm{E}-11$ & $\mathrm{Li}_{2} \mathrm{O}$ & 5.7E-06 & $\mathrm{Na}_{2} \mathrm{O}$ & 0.755 \\
\hline${ }^{15} 5 \mathrm{Eu}_{2} \mathrm{O}_{3}$ & $2.7 \mathrm{E}-12$ & $\mathrm{MgO}$ & 2.4E-04 & $\mathrm{NiO}$ & 3.6E-04 \\
\hline${ }^{226} \mathrm{RaO}$ & $1.9 \mathrm{E}-14$ & $\mathrm{MoO}_{3}$ & $2.1 \mathrm{E}-05$ & $\mathrm{PbO}$ & 0.00005 \\
\hline${ }^{227} \mathrm{Ac}_{2} \mathrm{O}_{3}$ & $3.6 \mathrm{E}-17$ & $\mathrm{Nd}_{2} \mathrm{O}_{3}$ & $0.0 \mathrm{E}+00$ & $\mathrm{P}_{2} \mathrm{O}_{5}$ & 0.0472 \\
\hline${ }^{228} \mathrm{RaO}$ & $5.8 \mathrm{E}-14$ & $\mathrm{PdO}$ & $0.0 \mathrm{E}+00$ & $\mathrm{SiO}_{2}$ & 0.0081 \\
\hline${ }^{229} \mathrm{ThO}_{2}$ & 9.3E-13 & $\mathrm{Pr}_{2} \mathrm{O}_{3}$ & $0.0 \mathrm{E}+00$ & $\mathrm{SO}_{3}$ & 0.0767 \\
\hline${ }^{231} \mathrm{~Pa}_{2} \mathrm{O}_{5}$ & $2.7 \mathrm{E}-10$ & $\mathrm{Rb}_{2} \mathrm{O}$ & $0.0 \mathrm{E}+00$ & $\mathrm{SrO}$ & $0.0 \mathrm{E}+00$ \\
\hline${ }^{232} \mathrm{ThO}_{2}$ & $8.1 \mathrm{E}-06$ & $\mathrm{Rh}_{2} \mathrm{O}_{3}$ & $0.0 \mathrm{E}+00$ & $\mathrm{ZrO}_{2}$ & $9.3 \mathrm{E}-06$ \\
\hline${ }^{232} \mathrm{UO}_{3}$ & $2.7 \mathrm{E}-13$ & $\mathrm{RuO}_{2}$ & $0.0 \mathrm{E}+00$ & \multirow{26}{*}{\multicolumn{2}{|c|}{$\begin{array}{l}\text { Note that the oxide weight } \\
\text { fractions reported above } \\
\text { and immediately to the left } \\
\text { are for the sum of stable } \\
\text { plus radioactive isotopes. }\end{array}$}} \\
\hline${ }^{233} \mathrm{UO}_{3}$ & $3.7 \mathrm{E}-08$ & $\mathrm{Sb}_{2} \mathrm{O}_{3}$ & 3.7E-05 & & \\
\hline${ }^{234} \mathrm{UO}_{3}$ & 2.3E-08 & $\mathrm{SeO}_{2}$ & 7.6E-07 & & \\
\hline${ }^{235} \mathrm{UO}_{3}$ & $2.9 \mathrm{E}-06$ & $\mathrm{Ta}_{2} \mathrm{O}_{5}$ & $0.0 \mathrm{E}+00$ & & \\
\hline${ }^{236} \mathrm{UO}_{3}$ & $3.0 \mathrm{E}-08$ & $\mathrm{TeO}_{2}$ & $0.0 \mathrm{E}+00$ & & \\
\hline${ }^{237} \mathrm{NpO}_{2}$ & $1.5 \mathrm{E}-07$ & $\mathrm{ThO}_{2}$ & $8.1 \mathrm{E}-06$ & & \\
\hline${ }^{238} \mathrm{PuO}_{2}$ & $4.2 \mathrm{E}-12$ & $\mathrm{TiO}_{2}$ & 9.5E-06 & & \\
\hline${ }^{238} \mathrm{UO}_{3}$ & $4.2 \mathrm{E}-04$ & $\mathrm{Tl}_{2} \mathrm{O}$ & $0.0 \mathrm{E}+00$ & & \\
\hline${ }^{239} \mathrm{PuO}_{2}$ & 6.6E-08 & $\mathrm{V}_{2} \mathrm{O}_{5}$ & $1.9 \mathrm{E}-05$ & & \\
\hline${ }^{240} \mathrm{PuO}_{2}$ & 2.7E-09 & $\mathrm{WO}_{3}$ & $0.0 \mathrm{E}+00$ & & \\
\hline${ }^{241} \mathrm{Am}_{2} \mathrm{O}_{3}$ & $1.4 \mathrm{E}-09$ & $\mathrm{Y}_{2} \mathrm{O}_{3}$ & $0.0 \mathrm{E}+00$ & & \\
\hline${ }^{241} \mathrm{PuO}_{2}$ & $3.0 \mathrm{E}-11$ & $\mathrm{ZnO}$ & 1.3E-04 & & \\
\hline${ }^{242} \mathrm{Cm}_{2} \mathrm{O}_{3}$ & $3.3 \mathrm{E}-15$ & & & & \\
\hline${ }^{242} \mathrm{PuO}_{2}$ & $7.5 \mathrm{E}-12$ & & & & \\
\hline${ }^{243} \mathrm{Am}_{2} \mathrm{O}_{3}$ & $6.7 \mathrm{E}-12$ & & & & \\
\hline${ }^{243} \mathrm{Cm}_{2} \mathrm{O}_{3}$ & $6.4 \mathrm{E}-15$ & & & & \\
\hline${ }^{244} \mathrm{Cm}_{2} \mathrm{O}_{3}$ & $9.6 \mathrm{E}-14$ & & & & \\
\hline${ }^{59} \mathrm{NiO}$ & $1.2 \mathrm{E}-07$ & & & & \\
\hline${ }^{60} \mathrm{CoO}$ & $1.4 \mathrm{E}-11$ & & & & \\
\hline${ }^{63} \mathrm{NiO}$ & $1.5 \mathrm{E}-08$ & & & & \\
\hline${ }^{79} \mathrm{SeO}_{2}$ & 5.7E-09 & & & & \\
\hline${ }^{90} \mathrm{SrO}$ & $0.0 \mathrm{E}+00$ & & & & \\
\hline${ }^{90} \mathrm{Y}_{2} \mathrm{O}_{3}$ & $1.2 \mathrm{E}-11$ & & & & \\
\hline${ }^{93} \mathrm{ZrO}_{2}$ & $2.0 \mathrm{E}-06$ & & & & \\
\hline${ }^{93} \mathrm{Nb}_{2} \mathrm{O}_{5}$ & $1.9 \mathrm{E}-11$ & & & & \\
\hline${ }^{99} \mathrm{Tc}_{2} \mathrm{O}_{7}$ & 4.4E-06 & & & & \\
\hline
\end{tabular}


Table 5.21. Cluster Y4 Waste Oxide Composition

\begin{tabular}{|c|c|c|c|c|c|}
\hline $\begin{array}{c}\text { Radioactive } \\
\text { oxides }\end{array}$ & $\begin{array}{l}\text { Wt. } \\
\text { frac. }\end{array}$ & $\begin{array}{c}\text { Supplemental } \\
\text { Species Oxides }\end{array}$ & Wt. frac. & $\begin{array}{c}\text { BBI Species } \\
\text { Oxides }\end{array}$ & Wt. frac. \\
\hline${ }^{106} \mathrm{RuO}_{2}$ & $7.1 \mathrm{E}-22$ & $\mathrm{Ag}_{2} \mathrm{O}$ & $0.0 \mathrm{E}+00$ & $\mathrm{Al}_{2} \mathrm{O}_{3}$ & 0.039 \\
\hline${ }^{113} \mathrm{CdO}$ & 1.6E-12 & $\mathrm{As}_{2} \mathrm{O}_{5}$ & $0.0 \mathrm{E}+00$ & $\mathrm{Bi}_{2} \mathrm{O}_{3}$ & $3.8 \mathrm{E}-06$ \\
\hline${ }^{12} 5 \mathrm{Sb}_{2} \mathrm{O}_{3}$ & $1.2 \mathrm{E}-12$ & $\mathrm{~B}_{2} \mathrm{O}_{3}$ & $0.0 \mathrm{E}+00$ & $\mathrm{CaO}$ & 0.00019 \\
\hline${ }^{126} \mathrm{SnO}_{2}$ & $1.9 \mathrm{E}-09$ & $\mathrm{BaO}$ & 9.9E-05 & $\mathrm{Cl}$ & 0.002 \\
\hline${ }^{129} \mathrm{I}$ & $2.8 \mathrm{E}-06$ & $\mathrm{BeO}$ & $6.2 \mathrm{E}-05$ & $\mathrm{Cr}_{2} \mathrm{O}_{3}$ & 0.0015 \\
\hline${ }^{134} \mathrm{Cs}_{2} \mathrm{O}$ & $1.2 \mathrm{E}-14$ & $\mathrm{CdO}$ & $6.0 \mathrm{E}-07$ & $\mathrm{~F}$ & 0.0924 \\
\hline${ }^{137} \mathrm{Cs}_{2} \mathrm{O}$ & 4.7E-07 & $\mathrm{Ce}_{2} \mathrm{O}_{3}$ & $0.0 \mathrm{E}+00$ & $\mathrm{Fe}_{2} \mathrm{O}_{3}$ & 0.00004 \\
\hline${ }^{137} \mathrm{BaO}$ & 7.6E-14 & $\mathrm{Co}_{2} \mathrm{O}_{3}$ & 2.4E-04 & $\mathrm{K}_{2} \mathrm{O}$ & 0.007 \\
\hline${ }^{151} \mathrm{Sm}_{2} \mathrm{O}_{3}$ & $3.5 \mathrm{E}-11$ & $\mathrm{Cs}_{2} \mathrm{O}$ & $0.0 \mathrm{E}+00$ & $\mathrm{La}_{2} \mathrm{O}_{3}$ & $3.0 \mathrm{E}-08$ \\
\hline${ }^{152} \mathrm{Eu}_{2} \mathrm{O}_{3}$ & $3.6 \mathrm{E}-13$ & $\mathrm{CuO}$ & $6.3 \mathrm{E}-05$ & $\mathrm{MnO}$ & $2.8 \mathrm{E}-06$ \\
\hline${ }^{154} \mathrm{Eu}_{2} \mathrm{O}_{3}$ & $1.6 \mathrm{E}-11$ & $\mathrm{Li}_{2} \mathrm{O}$ & $2.5 \mathrm{E}-04$ & $\mathrm{Na}_{2} \mathrm{O}$ & 0.595 \\
\hline${ }^{15} 5 \mathrm{Eu}_{2} \mathrm{O}_{3}$ & $3.6 \mathrm{E}-12$ & $\mathrm{MgO}$ & $0.0 \mathrm{E}+00$ & $\mathrm{NiO}$ & $1.5 \mathrm{E}-05$ \\
\hline${ }^{226} \mathrm{RaO}$ & $8.8 \mathrm{E}-15$ & $\mathrm{MoO}_{3}$ & $1.4 \mathrm{E}-04$ & $\mathrm{PbO}$ & 0.00005 \\
\hline${ }^{227} \mathrm{Ac}_{2} \mathrm{O}_{3}$ & $7.0 \mathrm{E}-17$ & $\mathrm{Nd}_{2} \mathrm{O}_{3}$ & $0.0 \mathrm{E}+00$ & $\mathrm{P}_{2} \mathrm{O}_{5}$ & 0.0883 \\
\hline${ }^{228} \mathrm{RaO}$ & $6.4 \mathrm{E}-14$ & $\mathrm{PdO}$ & $0.0 \mathrm{E}+00$ & $\mathrm{SiO}_{2}$ & 0.0124 \\
\hline${ }^{229} \mathrm{ThO}_{2}$ & $1.4 \mathrm{E}-11$ & $\mathrm{Pr}_{2} \mathrm{O}_{3}$ & $0.0 \mathrm{E}+00$ & $\mathrm{SO}_{3}$ & 0.1595 \\
\hline${ }^{231} \mathrm{~Pa}_{2} \mathrm{O}_{5}$ & $1.1 \mathrm{E}-10$ & $\mathrm{Rb}_{2} \mathrm{O}$ & $0.0 \mathrm{E}+00$ & $\mathrm{SrO}$ & $0.0 \mathrm{E}+00$ \\
\hline${ }^{232} \mathrm{ThO}_{2}$ & $1.2 \mathrm{E}-04$ & $\mathrm{Rh}_{2} \mathrm{O}_{3}$ & $0.0 \mathrm{E}+00$ & $\mathrm{ZrO}_{2}$ & $3.0 \mathrm{E}-06$ \\
\hline${ }^{232} \mathrm{UO}_{3}$ & $3.8 \mathrm{E}-13$ & $\mathrm{RuO}_{2}$ & $0.0 \mathrm{E}+00$ & \multirow{26}{*}{\multicolumn{2}{|c|}{$\begin{array}{l}\text { Note that the oxide weight } \\
\text { fractions reported above } \\
\text { and immediately to the left } \\
\text { are for the sum of stable } \\
\text { plus radioactive isotopes. }\end{array}$}} \\
\hline${ }^{233} \mathrm{UO}_{3}$ & 5.2E-08 & $\mathrm{Sb}_{2} \mathrm{O}_{3}$ & $3.2 \mathrm{E}-04$ & & \\
\hline${ }^{234} \mathrm{UO}_{3}$ & 5.5E-09 & $\mathrm{SeO}_{2}$ & $1.5 \mathrm{E}-04$ & & \\
\hline${ }^{235} \mathrm{UO}_{3}$ & 5.9E-07 & $\mathrm{Ta}_{2} \mathrm{O}_{5}$ & $0.0 \mathrm{E}+00$ & & \\
\hline${ }^{236} \mathrm{UO}_{3}$ & $1.3 \mathrm{E}-08$ & $\mathrm{TeO}_{2}$ & $0.0 \mathrm{E}+00$ & & \\
\hline${ }^{237} \mathrm{NpO}_{2}$ & $7.8 \mathrm{E}-08$ & $\mathrm{ThO}_{2}$ & $1.2 \mathrm{E}-04$ & & \\
\hline${ }^{238} \mathrm{PuO}_{2}$ & $0.0 \mathrm{E}+00$ & $\mathrm{TiO}_{2}$ & 7.4E-05 & & \\
\hline${ }^{238} \mathrm{UO}_{3}$ & 8.4E-05 & $\mathrm{Tl}_{2} \mathrm{O}$ & $0.0 \mathrm{E}+00$ & & \\
\hline${ }^{239} \mathrm{PuO}_{2}$ & $0.0 \mathrm{E}+00$ & $\mathrm{~V}_{2} \mathrm{O}_{5}$ & $4.3 \mathrm{E}-04$ & & \\
\hline${ }^{240} \mathrm{PuO}_{2}$ & $0.0 \mathrm{E}+00$ & $\mathrm{WO}_{3}$ & $0.0 \mathrm{E}+00$ & & \\
\hline${ }^{241} \mathrm{Am}_{2} \mathrm{O}_{3}$ & 2.3E-09 & $\mathrm{Y}_{2} \mathrm{O}_{3}$ & $0.0 \mathrm{E}+00$ & & \\
\hline${ }^{241} \mathrm{PuO}_{2}$ & $0.0 \mathrm{E}+00$ & $\mathrm{ZnO}$ & $1.7 \mathrm{E}-04$ & & \\
\hline${ }^{242} \mathrm{Cm}_{2} \mathrm{O}_{3}$ & $4.2 \mathrm{E}-17$ & & & & \\
\hline${ }^{242} \mathrm{PuO}_{2}$ & $0.0 \mathrm{E}+00$ & & & & \\
\hline${ }^{243} \mathrm{Am}_{2} \mathrm{O}_{3}$ & 1.5E-11 & & & & \\
\hline${ }^{243} \mathrm{Cm}_{2} \mathrm{O}_{3}$ & $8.8 \mathrm{E}-17$ & & & & \\
\hline${ }^{244} \mathrm{Cm}_{2} \mathrm{O}_{3}$ & $1.3 \mathrm{E}-15$ & & & & \\
\hline${ }^{59} \mathrm{NiO}$ & 2.4E-08 & & & & \\
\hline${ }^{60} \mathrm{CoO}$ & $1.2 \mathrm{E}-13$ & & & & \\
\hline${ }^{63} \mathrm{NiO}$ & $2.9 \mathrm{E}-09$ & & & & \\
\hline${ }^{79} \mathrm{SeO}_{2}$ & 5.0E-09 & & & & \\
\hline${ }^{90} \mathrm{SrO}$ & $0.0 \mathrm{E}+00$ & & & & \\
\hline${ }^{90} \mathrm{Y}_{2} \mathrm{O}_{3}$ & 7.7E-13 & & & & \\
\hline${ }^{93} \mathrm{ZrO}_{2}$ & $2.8 \mathrm{E}-07$ & & & & \\
\hline${ }^{93} \mathrm{Nb}_{2} \mathrm{O}_{5}$ & $1.4 \mathrm{E}-11$ & & & & \\
\hline${ }^{99} \mathrm{Tc}_{2} \mathrm{O}_{7}$ & 1.2E-06 & & & & \\
\hline
\end{tabular}


Table 5.22. Cluster Y5 Waste Oxide Composition

\begin{tabular}{|c|c|c|c|c|c|}
\hline $\begin{array}{c}\text { Radioactive } \\
\text { oxides }\end{array}$ & $\begin{array}{l}\text { Wt. } \\
\text { frac. }\end{array}$ & $\begin{array}{c}\text { Supplemental } \\
\text { Species Oxides }\end{array}$ & Wt. frac. & $\begin{array}{c}\text { BBI Species } \\
\text { Oxides }\end{array}$ & Wt. frac. \\
\hline${ }^{106} \mathrm{RuO}_{2}$ & $4.7 \mathrm{E}-19$ & $\mathrm{Ag}_{2} \mathrm{O}$ & $0.0 \mathrm{E}+00$ & $\mathrm{Al}_{2} \mathrm{O}_{3}$ & 0.025 \\
\hline${ }^{113} \mathrm{CdO}$ & $1.2 \mathrm{E}-10$ & $\mathrm{As}_{2} \mathrm{O}_{5}$ & $1.0 \mathrm{E}-04$ & $\mathrm{Bi}_{2} \mathrm{O}_{3}$ & $8.4 \mathrm{E}-05$ \\
\hline${ }^{12} 5 \mathrm{Sb}_{2} \mathrm{O}_{3}$ & $3.2 \mathrm{E}-12$ & $\mathrm{~B}_{2} \mathrm{O}_{3}$ & 3.5E-04 & $\mathrm{CaO}$ & 0.00113 \\
\hline${ }^{126} \mathrm{SnO}_{2}$ & $3.1 \mathrm{E}-09$ & $\mathrm{BaO}$ & $1.8 \mathrm{E}-05$ & $\mathrm{Cl}$ & 0.004 \\
\hline${ }^{129} \mathrm{I}$ & $1.4 \mathrm{E}-06$ & $\mathrm{BeO}$ & $1.7 \mathrm{E}-05$ & $\mathrm{Cr}_{2} \mathrm{O}_{3}$ & 0.0030 \\
\hline${ }^{134} \mathrm{Cs}_{2} \mathrm{O}$ & 3.6E-14 & $\mathrm{CdO}$ & $5.4 \mathrm{E}-06$ & $\mathrm{~F}$ & 0.0124 \\
\hline${ }^{137} \mathrm{Cs}_{2} \mathrm{O}$ & $8.1 \mathrm{E}-07$ & $\mathrm{Ce}_{2} \mathrm{O}_{3}$ & $0.0 \mathrm{E}+00$ & $\mathrm{Fe}_{2} \mathrm{O}_{3}$ & 0.00112 \\
\hline${ }^{137} \mathrm{BaO}$ & $1.3 \mathrm{E}-13$ & $\mathrm{Co}_{2} \mathrm{O}_{3}$ & $3.5 \mathrm{E}-05$ & $\mathrm{~K}_{2} \mathrm{O}$ & 0.005 \\
\hline${ }^{151} \mathrm{Sm}_{2} \mathrm{O}_{3}$ & $1.0 \mathrm{E}-11$ & $\mathrm{Cs}_{2} \mathrm{O}$ & $0.0 \mathrm{E}+00$ & $\mathrm{La}_{2} \mathrm{O}_{3}$ & $4.1 \mathrm{E}-08$ \\
\hline${ }^{152} \mathrm{Eu}_{2} \mathrm{O}_{3}$ & $9.4 \mathrm{E}-14$ & $\mathrm{CuO}$ & $1.8 \mathrm{E}-05$ & $\mathrm{MnO}$ & 1.6E-05 \\
\hline${ }^{154} \mathrm{Eu}_{2} \mathrm{O}_{3}$ & $4.2 \mathrm{E}-12$ & $\mathrm{Li}_{2} \mathrm{O}$ & $1.5 \mathrm{E}-05$ & $\mathrm{Na}_{2} \mathrm{O}$ & 0.835 \\
\hline${ }^{15} 5 \mathrm{Eu}_{2} \mathrm{O}_{3}$ & $9.5 \mathrm{E}-13$ & $\mathrm{MgO}$ & 3.7E-04 & $\mathrm{NiO}$ & $4.8 \mathrm{E}-05$ \\
\hline${ }^{226} \mathrm{RaO}$ & $3.8 \mathrm{E}-14$ & $\mathrm{MoO}_{3}$ & $4.0 \mathrm{E}-05$ & $\mathrm{PbO}$ & 0.00002 \\
\hline${ }^{227} \mathrm{Ac}_{2} \mathrm{O}_{3}$ & $1.1 \mathrm{E}-17$ & $\mathrm{Nd}_{2} \mathrm{O}_{3}$ & 7.8E-05 & $\mathrm{P}_{2} \mathrm{O}_{5}$ & 0.0470 \\
\hline${ }^{228} \mathrm{RaO}$ & $2.3 \mathrm{E}-14$ & $\mathrm{PdO}$ & $0.0 \mathrm{E}+00$ & $\mathrm{SiO}_{2}$ & 0.0059 \\
\hline${ }^{229} \mathrm{ThO}_{2}$ & 3.3E-14 & $\mathrm{Pr}_{2} \mathrm{O}_{3}$ & $0.0 \mathrm{E}+00$ & $\mathrm{SO}_{3}$ & 0.0587 \\
\hline${ }^{231} \mathrm{~Pa}_{2} \mathrm{O}_{5}$ & $1.1 \mathrm{E}-10$ & $\mathrm{Rb}_{2} \mathrm{O}$ & $0.0 \mathrm{E}+00$ & $\mathrm{SrO}$ & $0.0 \mathrm{E}+00$ \\
\hline${ }^{232} \mathrm{ThO}_{2}$ & $1.9 \mathrm{E}-07$ & $\mathrm{Rh}_{2} \mathrm{O}_{3}$ & $0.0 \mathrm{E}+00$ & $\mathrm{ZrO}_{2}$ & $1.8 \mathrm{E}-05$ \\
\hline${ }^{232} \mathrm{UO}_{3}$ & $1.1 \mathrm{E}-13$ & $\mathrm{RuO}_{2}$ & $0.0 \mathrm{E}+00$ & \multirow{26}{*}{\multicolumn{2}{|c|}{$\begin{array}{l}\text { Note that the oxide weight } \\
\text { fractions reported above } \\
\text { and immediately to the left } \\
\text { are for the sum of stable } \\
\text { plus radioactive isotopes. }\end{array}$}} \\
\hline${ }^{233} \mathrm{UO}_{3}$ & $1.5 \mathrm{E}-08$ & $\mathrm{Sb}_{2} \mathrm{O}_{3}$ & $4.8 \mathrm{E}-05$ & & \\
\hline${ }^{234} \mathrm{UO}_{3}$ & $1.9 \mathrm{E}-08$ & $\mathrm{SeO}_{2}$ & $3.8 \mathrm{E}-05$ & & \\
\hline${ }^{235} \mathrm{UO}_{3}$ & $2.4 \mathrm{E}-06$ & $\mathrm{Ta}_{2} \mathrm{O}_{5}$ & $0.0 \mathrm{E}+00$ & & \\
\hline${ }^{236} \mathrm{UO}_{3}$ & $2.9 \mathrm{E}-08$ & $\mathrm{TeO}_{2}$ & $0.0 \mathrm{E}+00$ & & \\
\hline${ }^{237} \mathrm{NpO}_{2}$ & $3.4 \mathrm{E}-07$ & $\mathrm{ThO}_{2}$ & $1.9 \mathrm{E}-07$ & & \\
\hline${ }^{238} \mathrm{PuO}_{2}$ & $2.0 \mathrm{E}-11$ & $\mathrm{TiO}_{2}$ & $3.6 \mathrm{E}-05$ & & \\
\hline${ }^{238} \mathrm{UO}_{3}$ & 3.5E-04 & $\mathrm{Tl}_{2} \mathrm{O}$ & $0.0 \mathrm{E}+00$ & & \\
\hline${ }^{239} \mathrm{PuO}_{2}$ & $1.8 \mathrm{E}-07$ & $\mathrm{~V}_{2} \mathrm{O}_{5}$ & 5.7E-05 & & \\
\hline${ }^{240} \mathrm{PuO}_{2}$ & 9.7E-09 & $\mathrm{WO}_{3}$ & $0.0 \mathrm{E}+00$ & & \\
\hline${ }^{241} \mathrm{Am}_{2} \mathrm{O}_{3}$ & $1.9 \mathrm{E}-09$ & $\mathrm{Y}_{2} \mathrm{O}_{3}$ & $0.0 \mathrm{E}+00$ & & \\
\hline${ }^{241} \mathrm{PuO}_{2}$ & $1.4 \mathrm{E}-10$ & $\mathrm{ZnO}$ & $6.2 \mathrm{E}-05$ & & \\
\hline${ }^{242} \mathrm{Cm}_{2} \mathrm{O}_{3}$ & $5.6 \mathrm{E}-14$ & & & & \\
\hline${ }^{242} \mathrm{PuO}_{2}$ & 3.5E-11 & & & & \\
\hline${ }^{243} \mathrm{Am}_{2} \mathrm{O}_{3}$ & $1.7 \mathrm{E}-11$ & & & & \\
\hline${ }^{243} \mathrm{Cm}_{2} \mathrm{O}_{3}$ & $2.1 \mathrm{E}-13$ & & & & \\
\hline${ }^{244} \mathrm{Cm}_{2} \mathrm{O}_{3}$ & $3.1 \mathrm{E}-12$ & & & & \\
\hline${ }^{59} \mathrm{NiO}$ & 8.6E-08 & & & & \\
\hline${ }^{60} \mathrm{CoO}$ & 7.7E-12 & & & & \\
\hline${ }^{63} \mathrm{NiO}$ & $1.0 \mathrm{E}-08$ & & & & \\
\hline${ }^{79} \mathrm{SeO}_{2}$ & 7.8E-09 & & & & \\
\hline${ }^{90} \mathrm{SrO}$ & $0.0 \mathrm{E}+00$ & & & & \\
\hline${ }^{90} \mathrm{Y}_{2} \mathrm{O}_{3}$ & $2.1 \mathrm{E}-11$ & & & & \\
\hline${ }^{93} \mathrm{ZrO}_{2}$ & $5.1 \mathrm{E}-06$ & & & & \\
\hline${ }^{93} \mathrm{Nb}_{2} \mathrm{O}_{5}$ & $3.6 \mathrm{E}-11$ & & & & \\
\hline${ }^{99} \mathrm{Tc}_{2} \mathrm{O}_{7}$ & 5.8E-06 & & & & \\
\hline
\end{tabular}


Table 5.23. Cluster Y6 Waste Oxide Composition

\begin{tabular}{|c|c|c|c|c|c|}
\hline $\begin{array}{c}\text { Radioactive } \\
\text { oxides }\end{array}$ & Wt. frac. & $\begin{array}{c}\text { Supplemental } \\
\text { Species Oxides }\end{array}$ & Wt. frac. & $\begin{array}{c}\text { BBI Species } \\
\text { Oxides }\end{array}$ & Wt. frac. \\
\hline${ }^{106} \mathrm{RuO}_{2}$ & $1.0 \mathrm{E}-23$ & $\mathrm{Ag}_{2} \mathrm{O}$ & $0.0 \mathrm{E}+00$ & $\mathrm{Al}_{2} \mathrm{O}_{3}$ & 0.001 \\
\hline${ }^{113} \mathrm{CdO}$ & $1.1 \mathrm{E}-12$ & $\mathrm{As}_{2} \mathrm{O}_{5}$ & $0.0 \mathrm{E}+00$ & $\mathrm{Bi}_{2} \mathrm{O}_{3}$ & $2.5 \mathrm{E}-04$ \\
\hline${ }^{12} 5 \mathrm{Sb}_{2} \mathrm{O}_{3}$ & $1.7 \mathrm{E}-15$ & $\mathrm{~B}_{2} \mathrm{O}_{3}$ & $2.1 \mathrm{E}-03$ & $\mathrm{CaO}$ & 0.00029 \\
\hline${ }^{126} \mathrm{SnO}_{2}$ & 5.9E-11 & $\mathrm{BaO}$ & 1.4E-04 & $\mathrm{Cl}$ & 0.002 \\
\hline${ }^{129} \mathrm{I}$ & $1.6 \mathrm{E}-07$ & $\mathrm{BeO}$ & $8.8 \mathrm{E}-05$ & $\mathrm{Cr}_{2} \mathrm{O}_{3}$ & 0.0002 \\
\hline${ }^{134} \mathrm{Cs}_{2} \mathrm{O}$ & $3.9 \mathrm{E}-17$ & $\mathrm{CdO}$ & 6.8E-06 & $\mathrm{F}$ & 0.0405 \\
\hline${ }^{137} \mathrm{Cs}_{2} \mathrm{O}$ & $1.5 \mathrm{E}-07$ & $\mathrm{Ce}_{2} \mathrm{O}_{3}$ & $0.0 \mathrm{E}+00$ & $\mathrm{Fe}_{2} \mathrm{O}_{3}$ & 0.00091 \\
\hline${ }^{137} \mathrm{BaO}$ & $2.4 \mathrm{E}-14$ & $\mathrm{Co}_{2} \mathrm{O}_{3}$ & 1.8E-04 & $\mathrm{K}_{2} \mathrm{O}$ & 0.001 \\
\hline${ }^{151} \mathrm{Sm}_{2} \mathrm{O}_{3}$ & $2.0 \mathrm{E}-12$ & $\mathrm{Cs}_{2} \mathrm{O}$ & $0.0 \mathrm{E}+00$ & $\mathrm{La}_{2} \mathrm{O}_{3}$ & $4.3 \mathrm{E}-15$ \\
\hline${ }^{152} \mathrm{Eu}_{2} \mathrm{O}_{3}$ & $6.0 \mathrm{E}-18$ & $\mathrm{CuO}$ & $1.5 \mathrm{E}-04$ & $\mathrm{MnO}$ & $2.4 \mathrm{E}-05$ \\
\hline${ }^{154} \mathrm{Eu}_{2} \mathrm{O}_{3}$ & $2.7 \mathrm{E}-16$ & $\mathrm{Li}_{2} \mathrm{O}$ & $0.0 \mathrm{E}+00$ & $\mathrm{Na}_{2} \mathrm{O}$ & 0.567 \\
\hline${ }^{15} 5 \mathrm{Eu}_{2} \mathrm{O}_{3}$ & 7.1E-17 & $\mathrm{MgO}$ & $0.0 \mathrm{E}+00$ & $\mathrm{NiO}$ & $4.4 \mathrm{E}-06$ \\
\hline${ }^{226} \mathrm{RaO}$ & $9.2 \mathrm{E}-16$ & $\mathrm{MoO}_{3}$ & $1.9 \mathrm{E}-04$ & $\mathrm{PbO}$ & 0.00004 \\
\hline${ }^{227} \mathrm{Ac}_{2} \mathrm{O}_{3}$ & $4.5 \mathrm{E}-17$ & $\mathrm{Nd}_{2} \mathrm{O}_{3}$ & $0.0 \mathrm{E}+00$ & $\mathrm{P}_{2} \mathrm{O}_{5}$ & 0.1076 \\
\hline${ }^{228} \mathrm{RaO}$ & 5.6E-15 & $\mathrm{PdO}$ & $0.0 \mathrm{E}+00$ & $\mathrm{SiO}_{2}$ & 0.0016 \\
\hline${ }^{229} \mathrm{ThO}_{2}$ & $1.0 \mathrm{E}-11$ & $\mathrm{Pr}_{2} \mathrm{O}_{3}$ & $0.0 \mathrm{E}+00$ & $\mathrm{SO}_{3}$ & 0.2711 \\
\hline${ }^{231} \mathrm{~Pa}_{2} \mathrm{O}_{5}$ & $8.3 \mathrm{E}-13$ & $\mathrm{Rb}_{2} \mathrm{O}$ & $0.0 \mathrm{E}+00$ & $\mathrm{SrO}$ & $0.0 \mathrm{E}+00$ \\
\hline${ }^{232} \mathrm{ThO}_{2}$ & $9.2 \mathrm{E}-05$ & $\mathrm{Rh}_{2} \mathrm{O}_{3}$ & $0.0 \mathrm{E}+00$ & $\mathrm{ZrO}_{2}$ & $1.6 \mathrm{E}-05$ \\
\hline${ }^{232} \mathrm{UO}_{3}$ & $1.8 \mathrm{E}-14$ & $\mathrm{RuO}_{2}$ & $0.0 \mathrm{E}+00$ & \multirow{26}{*}{\multicolumn{2}{|c|}{$\begin{array}{l}\text { Note that the oxide weight } \\
\text { fractions reported above } \\
\text { and immediately to the left } \\
\text { are for the sum of stable } \\
\text { plus radioactive isotopes. }\end{array}$}} \\
\hline${ }^{233} \mathrm{UO}_{3}$ & $2.5 \mathrm{E}-09$ & $\mathrm{Sb}_{2} \mathrm{O}_{3}$ & $0.0 \mathrm{E}+00$ & & \\
\hline${ }^{234} \mathrm{UO}_{3}$ & 2.6E-08 & $\mathrm{SeO}_{2}$ & $0.0 \mathrm{E}+00$ & & \\
\hline${ }^{235} \mathrm{UO}_{3}$ & $3.3 \mathrm{E}-06$ & $\mathrm{Ta}_{2} \mathrm{O}_{5}$ & $0.0 \mathrm{E}+00$ & & \\
\hline${ }^{236} \mathrm{UO}_{3}$ & $2.9 \mathrm{E}-08$ & $\mathrm{TeO}_{2}$ & $0.0 \mathrm{E}+00$ & & \\
\hline${ }^{237} \mathrm{NpO}_{2}$ & 5.3E-09 & $\mathrm{ThO}_{2}$ & 9.2E-05 & & \\
\hline${ }^{238} \mathrm{PuO}_{2}$ & $5.5 \mathrm{E}-12$ & $\mathrm{TiO}_{2}$ & $1.4 \mathrm{E}-04$ & & \\
\hline${ }^{238} \mathrm{UO}_{3}$ & $4.8 \mathrm{E}-04$ & $\mathrm{Tl}_{2} \mathrm{O}$ & $0.0 \mathrm{E}+00$ & & \\
\hline${ }^{239} \mathrm{PuO}_{2}$ & $1.7 \mathrm{E}-07$ & $\mathrm{~V}_{2} \mathrm{O}_{5}$ & 5.7E-04 & & \\
\hline${ }^{240} \mathrm{PuO}_{2}$ & $5.8 \mathrm{E}-09$ & $\mathrm{WO}_{3}$ & $0.0 \mathrm{E}+00$ & & \\
\hline${ }^{241} \mathrm{Am}_{2} \mathrm{O}_{3}$ & $2.4 \mathrm{E}-10$ & $\mathrm{Y}_{2} \mathrm{O}_{3}$ & $0.0 \mathrm{E}+00$ & & \\
\hline${ }^{241} \mathrm{PuO}_{2}$ & $3.2 \mathrm{E}-11$ & $\mathrm{ZnO}$ & $1.4 \mathrm{E}-03$ & & \\
\hline${ }^{242} \mathrm{Cm}_{2} \mathrm{O}_{3}$ & $3.2 \mathrm{E}-16$ & & & & \\
\hline${ }^{242} \mathrm{PuO}_{2}$ & $7.0 \mathrm{E}-12$ & & & & \\
\hline${ }^{243} \mathrm{Am}_{2} \mathrm{O}_{3}$ & $3.8 \mathrm{E}-13$ & & & & \\
\hline${ }^{243} \mathrm{Cm}_{2} \mathrm{O}_{3}$ & $2.5 \mathrm{E}-16$ & & & & \\
\hline${ }^{244} \mathrm{Cm}_{2} \mathrm{O}_{3}$ & $3.7 \mathrm{E}-15$ & & & & \\
\hline${ }^{59} \mathrm{NiO}$ & $1.1 \mathrm{E}-09$ & & & & \\
\hline${ }^{60} \mathrm{CoO}$ & $6.2 \mathrm{E}-13$ & & & & \\
\hline${ }^{63} \mathrm{NiO}$ & $1.3 \mathrm{E}-10$ & & & & \\
\hline${ }^{79} \mathrm{SeO}_{2}$ & $1.0 \mathrm{E}-10$ & & & & \\
\hline${ }^{90} \mathrm{SrO}$ & $0.0 \mathrm{E}+00$ & & & & \\
\hline${ }^{90} \mathrm{Y}_{2} \mathrm{O}_{3}$ & $1.9 \mathrm{E}-13$ & & & & \\
\hline${ }^{93} \mathrm{ZrO}_{2}$ & $6.0 \mathrm{E}-08$ & & & & \\
\hline${ }^{93} \mathrm{Nb}_{2} \mathrm{O}_{5}$ & $4.3 \mathrm{E}-12$ & & & & \\
\hline${ }^{99} \mathrm{Tc}_{2} \mathrm{O}_{7}$ & $2.5 \mathrm{E}-06$ & & & & \\
\hline
\end{tabular}


Table 5.24. Cluster Y7 Waste Oxide Composition

\begin{tabular}{|c|c|c|c|c|c|}
\hline $\begin{array}{c}\text { Radioactive } \\
\text { oxides }\end{array}$ & Wt. frac. & $\begin{array}{c}\text { Supplemental } \\
\text { Species Oxides }\end{array}$ & Wt. frac. & $\begin{array}{c}\text { BBI Species } \\
\text { Oxides }\end{array}$ & Wt. frac. \\
\hline${ }^{106} \mathrm{RuO}_{2}$ & $3.8 \mathrm{E}-19$ & $\mathrm{Ag}_{2} \mathrm{O}$ & $0.0 \mathrm{E}+00$ & $\mathrm{Al}_{2} \mathrm{O}_{3}$ & 0.006 \\
\hline${ }^{113} \mathrm{CdO}$ & $1.1 \mathrm{E}-10$ & $\mathrm{As}_{2} \mathrm{O}_{5}$ & $0.0 \mathrm{E}+00$ & $\mathrm{Bi}_{2} \mathrm{O}_{3}$ & $1.0 \mathrm{E}-06$ \\
\hline${ }^{12} 5 \mathrm{Sb}_{2} \mathrm{O}_{3}$ & $5.8 \mathrm{E}-13$ & $\mathrm{~B}_{2} \mathrm{O}_{3}$ & $0.0 \mathrm{E}+00$ & $\mathrm{CaO}$ & 0.00022 \\
\hline${ }^{126} \mathrm{SnO}_{2}$ & 2.7E-09 & $\mathrm{BaO}$ & $0.0 \mathrm{E}+00$ & $\mathrm{Cl}$ & 0.003 \\
\hline${ }^{129} \mathrm{I}$ & $5.9 \mathrm{E}-07$ & $\mathrm{BeO}$ & $0.0 \mathrm{E}+00$ & $\mathrm{Cr}_{2} \mathrm{O}_{3}$ & 0.0018 \\
\hline${ }^{134} \mathrm{Cs}_{2} \mathrm{O}$ & 2.9E-14 & $\mathrm{CdO}$ & $0.0 \mathrm{E}+00$ & F & 0.0580 \\
\hline${ }^{137} \mathrm{Cs}_{2} \mathrm{O}$ & $6.9 \mathrm{E}-07$ & $\mathrm{Ce}_{2} \mathrm{O}_{3}$ & $0.0 \mathrm{E}+00$ & $\mathrm{Fe}_{2} \mathrm{O}_{3}$ & 0.00063 \\
\hline${ }^{137} \mathrm{BaO}$ & $1.1 \mathrm{E}-13$ & $\mathrm{Co}_{2} \mathrm{O}_{3}$ & $0.0 \mathrm{E}+00$ & $\mathrm{~K}_{2} \mathrm{O}$ & 0.004 \\
\hline${ }^{151} \mathrm{Sm}_{2} \mathrm{O}_{3}$ & $2.1 \mathrm{E}-19$ & $\mathrm{Cs}_{2} \mathrm{O}$ & $0.0 \mathrm{E}+00$ & $\mathrm{La}_{2} \mathrm{O}_{3}$ & 2.7E-07 \\
\hline${ }^{152} \mathrm{Eu}_{2} \mathrm{O}_{3}$ & $5.1 \mathrm{E}-24$ & $\mathrm{CuO}$ & $0.0 \mathrm{E}+00$ & $\mathrm{MnO}$ & 4.9E-05 \\
\hline${ }^{154} \mathrm{Eu}_{2} \mathrm{O}_{3}$ & $3.4 \mathrm{E}-22$ & $\mathrm{Li}_{2} \mathrm{O}$ & $0.0 \mathrm{E}+00$ & $\mathrm{Na}_{2} \mathrm{O}$ & 0.639 \\
\hline${ }^{15} 5 \mathrm{Eu}_{2} \mathrm{O}_{3}$ & $8.8 \mathrm{E}-23$ & $\mathrm{MgO}$ & $0.0 \mathrm{E}+00$ & $\mathrm{NiO}$ & 4.6E-05 \\
\hline${ }^{226} \mathrm{RaO}$ & $3.5 \mathrm{E}-14$ & $\mathrm{MoO}_{3}$ & $0.0 \mathrm{E}+00$ & $\mathrm{PbO}$ & 0.00001 \\
\hline${ }^{227} \mathrm{Ac}_{2} \mathrm{O}_{3}$ & $1.2 \mathrm{E}-26$ & $\mathrm{Nd}_{2} \mathrm{O}_{3}$ & $0.0 \mathrm{E}+00$ & $\mathrm{P}_{2} \mathrm{O}_{5}$ & 0.2705 \\
\hline${ }^{228} \mathrm{RaO}$ & $1.2 \mathrm{E}-14$ & $\mathrm{PdO}$ & $0.0 \mathrm{E}+00$ & $\mathrm{SiO}_{2}$ & 0.0013 \\
\hline${ }^{229} \mathrm{ThO}_{2}$ & $9.7 \mathrm{E}-15$ & $\mathrm{Pr}_{2} \mathrm{O}_{3}$ & $0.0 \mathrm{E}+00$ & $\mathrm{SO}_{3}$ & 0.0154 \\
\hline${ }^{231} \mathrm{~Pa}_{2} \mathrm{O}_{5}$ & $5.6 \mathrm{E}-11$ & $\mathrm{Rb}_{2} \mathrm{O}$ & $0.0 \mathrm{E}+00$ & $\mathrm{SrO}$ & $0.0 \mathrm{E}+00$ \\
\hline${ }^{232} \mathrm{ThO}_{2}$ & $6.8 \mathrm{E}-08$ & $\mathrm{Rh}_{2} \mathrm{O}_{3}$ & $0.0 \mathrm{E}+00$ & $\mathrm{ZrO}_{2}$ & 1.9E-05 \\
\hline${ }^{232} \mathrm{UO}_{3}$ & 6.5E-14 & $\mathrm{RuO}_{2}$ & $0.0 \mathrm{E}+00$ & \multirow{26}{*}{\multicolumn{2}{|c|}{$\begin{array}{l}\text { Note that the oxide weight } \\
\text { fractions reported above } \\
\text { and immediately to the left } \\
\text { are for the sum of stable } \\
\text { plus radioactive isotopes. }\end{array}$}} \\
\hline${ }^{233} \mathrm{UO}_{3}$ & $8.8 \mathrm{E}-09$ & $\mathrm{Sb}_{2} \mathrm{O}_{3}$ & $0.0 \mathrm{E}+00$ & & \\
\hline${ }^{234} \mathrm{UO}_{3}$ & 4.5E-09 & $\mathrm{SeO}_{2}$ & $0.0 \mathrm{E}+00$ & & \\
\hline${ }^{235} \mathrm{UO}_{3}$ & $5.4 \mathrm{E}-07$ & $\mathrm{Ta}_{2} \mathrm{O}_{5}$ & $0.0 \mathrm{E}+00$ & & \\
\hline${ }^{236} \mathrm{UO}_{3}$ & $1.0 \mathrm{E}-08$ & $\mathrm{TeO}_{2}$ & $0.0 \mathrm{E}+00$ & & \\
\hline${ }^{237} \mathrm{NpO}_{2}$ & $2.5 \mathrm{E}-07$ & $\mathrm{ThO}_{2}$ & 6.8E-08 & & \\
\hline${ }^{238} \mathrm{PuO}_{2}$ & $6.9 \mathrm{E}-10$ & $\mathrm{TiO}_{2}$ & $0.0 \mathrm{E}+00$ & & \\
\hline${ }^{238} \mathrm{UO}_{3}$ & 7.6E-05 & $\mathrm{Tl}_{2} \mathrm{O}$ & $0.0 \mathrm{E}+00$ & & \\
\hline${ }^{239} \mathrm{PuO}_{2}$ & 5.4E-06 & $\mathrm{V}_{2} \mathrm{O}_{5}$ & $0.0 \mathrm{E}+00$ & & \\
\hline${ }^{240} \mathrm{PuO}_{2}$ & $3.1 \mathrm{E}-07$ & $\mathrm{WO}_{3}$ & $0.0 \mathrm{E}+00$ & & \\
\hline${ }^{241} \mathrm{Am}_{2} \mathrm{O}_{3}$ & $4.8 \mathrm{E}-08$ & $\mathrm{Y}_{2} \mathrm{O}_{3}$ & $0.0 \mathrm{E}+00$ & & \\
\hline${ }^{241} \mathrm{PuO}_{2}$ & $4.8 \mathrm{E}-09$ & $\mathrm{ZnO}$ & $0.0 \mathrm{E}+00$ & & \\
\hline${ }^{242} \mathrm{Cm}_{2} \mathrm{O}_{3}$ & 7.3E-13 & & & & \\
\hline${ }^{242} \mathrm{PuO}_{2}$ & $1.2 \mathrm{E}-09$ & & & & \\
\hline${ }^{243} \mathrm{Am}_{2} \mathrm{O}_{3}$ & $4.4 \mathrm{E}-10$ & & & & \\
\hline${ }^{243} \mathrm{Cm}_{2} \mathrm{O}_{3}$ & $1.7 \mathrm{E}-12$ & & & & \\
\hline${ }^{244} \mathrm{Cm}_{2} \mathrm{O}_{3}$ & $2.5 \mathrm{E}-11$ & & & & \\
\hline${ }^{59} \mathrm{NiO}$ & 7.3E-08 & & & & \\
\hline${ }^{60} \mathrm{CoO}$ & $6.0 \mathrm{E}-12$ & & & & \\
\hline${ }^{63} \mathrm{NiO}$ & $8.9 \mathrm{E}-09$ & & & & \\
\hline${ }^{79} \mathrm{SeO}_{2}$ & $6.8 \mathrm{E}-09$ & & & & \\
\hline${ }^{90} \mathrm{SrO}$ & $0.0 \mathrm{E}+00$ & & & & \\
\hline${ }^{90} \mathrm{Y}_{2} \mathrm{O}_{3}$ & $4.2 \mathrm{E}-10$ & & & & \\
\hline${ }^{93} \mathrm{ZrO}_{2}$ & $3.4 \mathrm{E}-06$ & & & & \\
\hline${ }^{93} \mathrm{Nb}_{2} \mathrm{O}_{5}$ & $1.7 \mathrm{E}-11$ & & & & \\
\hline${ }^{99} \mathrm{Tc}_{2} \mathrm{O}_{7}$ & 3.3E-06 & & & & \\
\hline
\end{tabular}


Table 5.25. Cluster Y8 Waste Oxide Composition

\begin{tabular}{|c|c|c|c|c|c|}
\hline $\begin{array}{c}\text { Radioactive } \\
\text { oxides }\end{array}$ & Wt. frac. & $\begin{array}{c}\text { Supplemental } \\
\text { Species Oxides }\end{array}$ & Wt. frac. & $\begin{array}{l}\text { BBI Species } \\
\text { Oxides }\end{array}$ & Wt. frac. \\
\hline${ }^{106} \mathrm{RuO}_{2}$ & $1.2 \mathrm{E}-22$ & $\mathrm{Ag}_{2} \mathrm{O}$ & $0.0 \mathrm{E}+00$ & $\mathrm{Al}_{2} \mathrm{O}_{3}$ & 0.002 \\
\hline${ }^{113} \mathrm{CdO}$ & $9.0 \mathrm{E}-13$ & $\mathrm{As}_{2} \mathrm{O}_{5}$ & $0.0 \mathrm{E}+00$ & $\mathrm{Bi}_{2} \mathrm{O}_{3}$ & $9.4 \mathrm{E}-05$ \\
\hline${ }^{12} 5 \mathrm{Sb}_{2} \mathrm{O}_{3}$ & 7.2E-17 & $\mathrm{B}_{2} \mathrm{O}_{3}$ & $0.0 \mathrm{E}+00$ & $\mathrm{CaO}$ & 0.00233 \\
\hline${ }^{126} \mathrm{SnO}_{2}$ & $1.1 \mathrm{E}-11$ & $\mathrm{BaO}$ & $0.0 \mathrm{E}+00$ & $\mathrm{Cl}$ & 0.002 \\
\hline${ }^{129} \mathrm{I}$ & $1.6 \mathrm{E}-07$ & $\mathrm{BeO}$ & 1.3E-04 & $\mathrm{Cr}_{2} \mathrm{O}_{3}$ & 0.0010 \\
\hline${ }^{134} \mathrm{Cs}_{2} \mathrm{O}$ & 2.2E-17 & $\mathrm{CdO}$ & $9.8 \mathrm{E}-06$ & $\mathrm{~F}$ & 0.0581 \\
\hline${ }^{137} \mathrm{Cs}_{2} \mathrm{O}$ & $1.6 \mathrm{E}-08$ & $\mathrm{Ce}_{2} \mathrm{O}_{3}$ & $0.0 \mathrm{E}+00$ & $\mathrm{Fe}_{2} \mathrm{O}_{3}$ & 0.00008 \\
\hline${ }^{137} \mathrm{BaO}$ & $2.5 \mathrm{E}-15$ & $\mathrm{Co}_{2} \mathrm{O}_{3}$ & $0.0 \mathrm{E}+00$ & $\mathrm{~K}_{2} \mathrm{O}$ & 0.001 \\
\hline${ }^{151} \mathrm{Sm}_{2} \mathrm{O}_{3}$ & $5.1 \mathrm{E}-13$ & $\mathrm{Cs}_{2} \mathrm{O}$ & $0.0 \mathrm{E}+00$ & $\mathrm{La}_{2} \mathrm{O}_{3}$ & $1.2 \mathrm{E}-07$ \\
\hline${ }^{152} \mathrm{Eu}_{2} \mathrm{O}_{3}$ & $1.0 \mathrm{E}-15$ & $\mathrm{CuO}$ & $0.0 \mathrm{E}+00$ & $\mathrm{MnO}$ & $3.4 \mathrm{E}-05$ \\
\hline${ }^{154} \mathrm{Eu}_{2} \mathrm{O}_{3}$ & $4.5 \mathrm{E}-14$ & $\mathrm{Li}_{2} \mathrm{O}$ & $0.0 \mathrm{E}+00$ & $\mathrm{Na}_{2} \mathrm{O}$ & 0.608 \\
\hline${ }^{15} 5 \mathrm{Eu}_{2} \mathrm{O}_{3}$ & $1.3 \mathrm{E}-14$ & $\mathrm{MgO}$ & $0.0 \mathrm{E}+00$ & $\mathrm{NiO}$ & 9.7E-06 \\
\hline${ }^{226} \mathrm{RaO}$ & $2.7 \mathrm{E}-14$ & $\mathrm{MoO}_{3}$ & $0.0 \mathrm{E}+00$ & $\mathrm{PbO}$ & 0.00006 \\
\hline${ }^{227} \mathrm{Ac}_{2} \mathrm{O}_{3}$ & $2.8 \mathrm{E}-17$ & $\mathrm{Nd}_{2} \mathrm{O}_{3}$ & $0.0 \mathrm{E}+00$ & $\mathrm{P}_{2} \mathrm{O}_{5}$ & 0.1308 \\
\hline${ }^{228} \mathrm{RaO}$ & $1.1 \mathrm{E}-13$ & PdO & $0.0 \mathrm{E}+00$ & $\mathrm{SiO}_{2}$ & 0.0055 \\
\hline${ }^{229} \mathrm{ThO}_{2}$ & $2.5 \mathrm{E}-12$ & $\mathrm{Pr}_{2} \mathrm{O}_{3}$ & $0.0 \mathrm{E}+00$ & $\mathrm{SO}_{3}$ & 0.1889 \\
\hline${ }^{231} \mathrm{~Pa}_{2} \mathrm{O}_{5}$ & $2.5 \mathrm{E}-12$ & $\mathrm{Rb}_{2} \mathrm{O}$ & $0.0 \mathrm{E}+00$ & $\mathrm{SrO}$ & $0.0 \mathrm{E}+00$ \\
\hline${ }^{232} \mathrm{ThO}_{2}$ & 2.2E-05 & $\mathrm{Rh}_{2} \mathrm{O}_{3}$ & $0.0 \mathrm{E}+00$ & $\mathrm{ZrO}_{2}$ & $3.0 \mathrm{E}-05$ \\
\hline${ }^{232} \mathrm{UO}_{3}$ & $2.5 \mathrm{E}-13$ & $\mathrm{RuO}_{2}$ & $0.0 \mathrm{E}+00$ & \multirow{26}{*}{\multicolumn{2}{|c|}{$\begin{array}{l}\text { Note that the oxide weight } \\
\text { fractions reported above } \\
\text { and immediately to the left } \\
\text { are for the sum of stable } \\
\text { plus radioactive isotopes. }\end{array}$}} \\
\hline${ }^{233} \mathrm{UO}_{3}$ & $3.5 \mathrm{E}-08$ & $\mathrm{Sb}_{2} \mathrm{O}_{3}$ & $0.0 \mathrm{E}+00$ & & \\
\hline${ }^{234} \mathrm{UO}_{3}$ & $1.2 \mathrm{E}-08$ & $\mathrm{SeO}_{2}$ & $0.0 \mathrm{E}+00$ & & \\
\hline${ }^{235} \mathrm{UO}_{3}$ & $1.4 \mathrm{E}-06$ & $\mathrm{Ta}_{2} \mathrm{O}_{5}$ & $0.0 \mathrm{E}+00$ & & \\
\hline${ }^{236} \mathrm{UO}_{3}$ & 2.2E-08 & $\mathrm{TeO}_{2}$ & $0.0 \mathrm{E}+00$ & & \\
\hline${ }^{237} \mathrm{NpO}_{2}$ & $3.8 \mathrm{E}-10$ & $\mathrm{ThO}_{2}$ & $2.2 \mathrm{E}-05$ & & \\
\hline${ }^{238} \mathrm{PuO}_{2}$ & $2.2 \mathrm{E}-13$ & $\mathrm{TiO}_{2}$ & $0.0 \mathrm{E}+00$ & & \\
\hline${ }^{238} \mathrm{UO}_{3}$ & $2.1 \mathrm{E}-04$ & $\mathrm{Tl}_{2} \mathrm{O}$ & $0.0 \mathrm{E}+00$ & & \\
\hline${ }^{239} \mathrm{PuO}_{2}$ & 7.5E-09 & $\mathrm{V}_{2} \mathrm{O}_{5}$ & $0.0 \mathrm{E}+00$ & & \\
\hline${ }^{240} \mathrm{PuO}_{2}$ & $2.4 \mathrm{E}-10$ & $\mathrm{WO}_{3}$ & $0.0 \mathrm{E}+00$ & & \\
\hline${ }^{241} \mathrm{Am}_{2} \mathrm{O}_{3}$ & $4.0 \mathrm{E}-12$ & $\mathrm{Y}_{2} \mathrm{O}_{3}$ & $0.0 \mathrm{E}+00$ & & \\
\hline${ }^{241} \mathrm{PuO}_{2}$ & $1.1 \mathrm{E}-12$ & $\mathrm{ZnO}$ & $0.0 \mathrm{E}+00$ & & \\
\hline${ }^{242} \mathrm{Cm}_{2} \mathrm{O}_{3}$ & $2.2 \mathrm{E}-17$ & & & & \\
\hline${ }^{242} \mathrm{PuO}_{2}$ & $2.4 \mathrm{E}-13$ & & & & \\
\hline${ }^{243} \mathrm{Am}_{2} \mathrm{O}_{3}$ & 5.6E-15 & & & & \\
\hline${ }^{243} \mathrm{Cm}_{2} \mathrm{O}_{3}$ & $1.7 \mathrm{E}-17$ & & & & \\
\hline${ }^{244} \mathrm{Cm}_{2} \mathrm{O}_{3}$ & $2.5 \mathrm{E}-16$ & & & & \\
\hline${ }^{59} \mathrm{NiO}$ & $4.2 \mathrm{E}-10$ & & & & \\
\hline${ }^{60} \mathrm{CoO}$ & 2.7E-14 & & & & \\
\hline${ }^{63} \mathrm{NiO}$ & $5.2 \mathrm{E}-11$ & & & & \\
\hline${ }^{79} \mathrm{SeO}_{2}$ & 6.6E-11 & & & & \\
\hline${ }^{90} \mathrm{SrO}$ & $0.0 \mathrm{E}+00$ & & & & \\
\hline${ }^{90} \mathrm{Y}_{2} \mathrm{O}_{3}$ & $1.3 \mathrm{E}-12$ & & & & \\
\hline${ }^{93} \mathrm{ZrO}_{2}$ & 5.3E-08 & & & & \\
\hline${ }^{93} \mathrm{Nb}_{2} \mathrm{O}_{5}$ & $3.5 \mathrm{E}-14$ & & & & \\
\hline${ }^{99} \mathrm{Tc}_{2} \mathrm{O}_{7}$ & $1.5 \mathrm{E}-07$ & & & & \\
\hline
\end{tabular}




\subsection{Glass Production Estimates}

This section provides estimates of the glass mass that ICV will produce from each of the waste clusters described in Sections 4 and 5. Section 6.1 summarizes existing data related to waste chemistry limits for LAW glass (ICV product, in particular). Section 6.2 gives estimates of the glass mass ICV will produce from each waste cluster according to "conservative," "best-estimate," and "stretch" assumptions for acceptable glass compositions. Two different combinations of soil and additives are considered.

\subsection{Glass Processing and Property Constraints}

For a Hanford LAW to be successfully processed with the ICV technology, it must make a glass that meets a number of product-quality and processing-related constraints and requirements. These fall into the following categories:

- Durability (the glass’s resistance to degradation by various environments)

- Regulatory compliance

- Electrical conductivity and viscosity consistent with process requirements

- Absence of gross phase separation.

The long-term immobilization of hazardous and radioactive components of Hanford LAW requires a glass with sufficient chemical durability. The durability-related requirements of the Hanford Integrated Disposal Facility (IDF) include the product consistency test (PCT, ASTM 2002) response and the vapor hydration test (VHT) (Vienna et al. 2001) response, which should be below 2 grams of glass dissolved per square meter of glass surface (for PCT) and below 50 grams of glass altered per square meter of glass surface per day (for VHT). If these durability-related constraints are met, then there is low risk that the vitrified product will cause the IDF performance assessment to fail the regulatory limits (Mann et al. 2001).

The ICV product may be required to meet certain toxicity characterization leach procedure (TCLP) (EPA 1997) responses to qualify for a variance or exclusion from Land Disposal Restrictions (LDR). Although the exact requirements for variance or exclusion from LDR are not yet defined, it is assumed that the most restrictive constraints placed on the glass are given by the universal treatment standards (UTS, 61 FR 2338, 62 FR 26041). To meet the UTS limits, the glass must be inherently resistant to the TCLP (e.g., have a low TCLP response), have low concentrations of hazardous and/or toxic components, or both.

The ICV process supplies heat by passing a current through the glass melt (i.e., Joule-heating). The electrical conductivity of the melt must fall within a certain range for successful heating. ICV has been successfully demonstrated with melt conductivities as high as 100 S/m (AMEC 2003, Kim et al. 2003) and as low as $\sim 0.3 \mathrm{~S} / \mathrm{m}$ (Buelt et al. 1987). In addition, the viscosity of the melt must be sufficient to allow for adequate melt homogenization without causing significant seepage from the melt contact refractories. Conductivity and viscosity are strongly influenced by and can be controlled by melt composition and temperature. This makes the ICV process relatively flexible to the concentrations of 
conductivity and viscosity influencing components. Experience has shown that viscosities near $10 \mathrm{~Pa} \cdot \mathrm{s}$ and temperatures between 1100 and $1500^{\circ} \mathrm{C}$ are the most suitable for processing LAW by ICV .

The formation of additional phases during ICV may be of concern for product quality. For example, excessive crystallization or soluble salt segregation could result in the release of hazardous or radioactive components through the formation of less durable phases. Multi-phase products are, however, acceptable as long as the hazardous and radioactive components are sufficiently immobilized.

All of the above properties are strongly influenced by the composition of the melter feed/glass. For example, alkali components modify the glass structure in ways that can decrease chemical durability and melt viscosity. To determine the acceptable waste loading, the influence of key waste components on glass properties and processing was considered. The waste compositions listed in Tables 5.1 through 5.25 were reviewed to determine which components would be most influential in determining the likely loading of the waste into ICV product glass. The components with concentrations higher than $1 \%$ (on a glass component mass basis) in at least one waste include $\mathrm{Al}_{2} \mathrm{O}_{3}, \mathrm{CaO}, \mathrm{Cl}, \mathrm{Cr}_{2} \mathrm{O}_{3}, \mathrm{~F}, \mathrm{Fe}_{2} \mathrm{O}_{3}, \mathrm{~K}_{2} \mathrm{O}, \mathrm{P}_{2} \mathrm{O}_{5}$, $\mathrm{SeO}_{2}, \mathrm{SiO}_{2}, \mathrm{SO}_{3}$, and $\mathrm{UO}_{3}$. These make up the key components, with two exceptions. The first is $\mathrm{SeO}_{2}$, whose maximum concentrations were derived from high detection limits, as discussed in Section 2, and which does not fall into the key-component category when the concentrations based on detection limits are removed. The second is $\mathrm{UO}_{3}$, whose maximum concentration was just above $1 \%$ on a waste mass basis; this would be a small fraction of a percent on a glass mole basis, making $\mathrm{UO}_{3}$ an insignificant contributor chemically. Of the key waste components, $\mathrm{Na}_{2} \mathrm{O}$ was found to be the most abundant component, accounting for up to $94 \%$ of the waste, followed by $\mathrm{Al}_{2} \mathrm{O}_{3}, \mathrm{P}_{2} \mathrm{O}_{5}$, and $\mathrm{SO}_{3}$, each accounting for more than $25 \%$ of at least one waste. In addition to the key waste components, $\mathrm{ZrO}_{2}$ and $\mathrm{B}_{2} \mathrm{O}_{3}$ were included in the evaluation as key glass components coming from both additives and waste.

Table 6.1 summarizes the qualitative effects of the key waste components on the properties of sodaaluminosilicate glasses such as those used in recent ICV testing and demonstrations. Many components have non-linear effects on glass properties. For example, $\mathrm{Al}_{2} \mathrm{O}_{3}$ and $\mathrm{B}_{2} \mathrm{O}_{3}$ decrease VHT response when added in relatively small concentrations but increase VHT response when added in relatively large concentrations. Other components tend to favorably influence one property while unfavorably influencing another. This makes glass formulation optimization a balancing act. If mathematical models existed with which to predict each key glass property as a function of composition, the optimum glass composition with a weighted balance of glass properties could be calculated for each waste or waste blend composition. Although models do exist to predict the values of some glass properties as functions of compositions (e.g., PCT response), models are not currently available to reliably predict many of the key glass properties as functions of composition (e.g., VHT response, crystallization potential, and salt accumulation). Therefore, component concentration limits were set based on a review of glass data that have been generated in support of the ST project along with data from other pertinent projects and general glass chemistry knowledge.

A total of 49 simulated LAW glasses have been fabricated and characterized as part of four studies in support of the ICV process. These 49 glasses make up the bulk of data most applicable to determining the constraints that dictate the loading of various Hanford low-activity wastes in the ICV product. The detailed compositions and results for these glasses are summarized in Table 6.2. These data do not cover all the compositions required to effectively immobilize the broad composition range of LAW (as 
Table 6.1. Summary of Key Component Effects on Soda-Aluminosilicate Glass Properties

\begin{tabular}{|c|c|c|c|c|c|c|c|c|}
\hline Component & VHT & PCT & TCLP & $\begin{array}{l}\text { Conduc- } \\
\text { tivity }\end{array}$ & Viscosity & $\begin{array}{l}\text { Crystal- } \\
\text { lization }\end{array}$ & Salt & Comment \\
\hline $\mathbf{A l}_{2} \mathbf{O}_{3}$ & $\downarrow \uparrow$ & $\downarrow$ & $\downarrow$ & $\leftrightarrow$ & $\uparrow$ & $\uparrow$ & $\leftrightarrow$ & $\begin{array}{c}\text { strongly increases VHT in high } \\
\text { concentrations }\end{array}$ \\
\hline $\mathbf{B}_{2} \mathbf{O}_{3}$ & $\downarrow \uparrow$ & $\downarrow \uparrow$ & $\uparrow$ & $\leftrightarrow$ & $\downarrow$ & $\downarrow$ & $\leftrightarrow$ & \begin{tabular}{|c|} 
reduces VHT and PCT at \\
small concentrations, additive
\end{tabular} \\
\hline $\mathrm{CaO}$ & $\uparrow$ & $\downarrow$ & $\uparrow$ & $\uparrow$ & $\downarrow$ & $\uparrow$ & $\downarrow$ & \\
\hline $\mathrm{Cl}$ & $\leftrightarrow$ & $\leftrightarrow$ & $\leftrightarrow$ & $\uparrow$ & $\leftrightarrow$ & $\leftrightarrow$ & $\uparrow$ & \\
\hline $\mathrm{Cr}_{2} \mathrm{O}_{3}$ & $\leftrightarrow$ & $\uparrow$ & $\uparrow$ & $\leftrightarrow$ & $\leftrightarrow$ & $\uparrow$ & $\uparrow$ & $\begin{array}{c}\text { increases TCLP only for } \mathrm{Cr} \\
\text { release }\end{array}$ \\
\hline $\mathbf{F}$ & $\leftrightarrow$ & $\downarrow$ & $\leftrightarrow$ & $\uparrow$ & $\downarrow$ & $\uparrow$ & $\uparrow$ & \\
\hline $\mathrm{Fe}_{2} \mathrm{O}_{3}$ & $\leftrightarrow$ & $\downarrow$ & $\downarrow$ & $\leftrightarrow$ & $\leftrightarrow$ & $\uparrow$ & $\leftrightarrow$ & \\
\hline $\mathbf{K}_{2} \mathbf{O}$ & $\uparrow$ & $\uparrow$ & $\uparrow$ & $\uparrow$ & $\downarrow$ & $\downarrow$ & $\downarrow$ & \\
\hline $\mathrm{Na}_{2} \mathrm{O}$ & $\uparrow$ & $\uparrow$ & $\uparrow$ & $\uparrow$ & $\downarrow$ & $\downarrow$ & $\downarrow$ & $\begin{array}{l}\text { main component of most } \\
\text { Hanford LAW }\end{array}$ \\
\hline $\mathbf{P}_{2} \mathbf{O}_{5}$ & $\uparrow$ & $\downarrow$ & $\leftrightarrow$ & $\leftrightarrow$ & $\leftrightarrow$ & $\uparrow$ & $\uparrow$ & $\begin{array}{c}\text { promotes amorphous phase } \\
\text { separation }\end{array}$ \\
\hline $\mathrm{SiO}_{2}$ & $\downarrow$ & $\downarrow$ & $\downarrow$ & $\downarrow$ & $\uparrow$ & $\downarrow$ & $\leftrightarrow$ & additive \\
\hline $\mathrm{SO}_{3}$ & $\leftrightarrow$ & $\leftrightarrow$ & $\leftrightarrow$ & $\leftrightarrow$ & $\leftrightarrow$ & $\leftrightarrow$ & $\uparrow$ & \\
\hline $\mathrm{ZrO}_{2}$ & $\downarrow$ & $\downarrow$ & $\downarrow$ & $\leftrightarrow$ & $\uparrow$ & $\uparrow$ & $\leftrightarrow$ & additive \\
\hline
\end{tabular}

described in Section 3). Thus, there is considerable uncertainty in the glass composition limits that determine the loading of these wastes in the ICV product.

To express this uncertainty, the glass composition constraints were set to describe three different cases: 1) a conservative estimate that can reasonably be expected to be successful, 2) a best estimate of the likely loading of each waste, and 3) a stretch or maximum loading case. The latter could be accomplished with sufficient investment in technology development. The constraints associated with each of these cases are described below, component by component.

$\mathrm{SiO}_{2} \quad$ A review of the glass data in Table 6.2 suggests that a lower limit to the $\mathrm{SiO}_{2}$ concentration in glass is needed. Nine of the ten glasses with $\mathrm{SiO}_{2}$ concentrations below $40 \mathrm{wt} \%$ either showed gross crystallinity after slow cooling or failed at least one product constraint. Therefore, for the conservative and best cases a lower silica limit of $40 \mathrm{wt} \%$ was used. Because at least one glass with $39 \mathrm{wt} \% \mathrm{SiO}_{2}$ passed all constraints and did not exhibit significant crystallization after slow cooling (AMP2-05), it is likely that with further testing glasses with $\mathrm{SiO}_{2}$ as low as $39 \mathrm{wt} \%$ can be successful. The stretch case $\mathrm{SiO}_{2}$ lower limit was set at $39 \mathrm{wt} \%$.

After excluding the 10 glasses in Table 6.2 that contained $\mathrm{SiO}_{2}$ concentrations of less than $40 \mathrm{wt} \%$, a tree analysis of the remaining 39 data points was performed. The purpose of this analysis was to separate 
the glasses that passed ("P") all product quality constraints, from those that failed ("F") at least one constraint. The tree analysis results are shown in Figure 6.1. Of the 39 glasses with $\mathrm{SiO}_{2} \geq 40 \mathrm{wt} \%, 20$ passed all constraints. Ten glasses with $\mathrm{Na}_{2} \mathrm{O} \geq 23 \mathrm{wt} \%$ failed at least one constraint. Of the 29 remaining glasses, 15 have $\mathrm{ZrO}_{2}$ concentrations $\geq 6.37 \mathrm{wt} \%$, all of which pass every constraint. Of the remaining 14 glasses, the eight with $\mathrm{Al}_{2} \mathrm{O}_{3}$ concentrations $\geq 9.46 \mathrm{wt} \%$ fail at least one constraint and all but one glass with $\mathrm{Al}_{2} \mathrm{O}_{3}<9.46 \mathrm{wt} \%$ pass every constraint.

$\mathrm{Na}_{2} \mathrm{O}$ Based on the tree analysis and results from studies on Hanford LAW glasses from other programs (primarily from Vienna et al. 2001), an upper limit for $\mathrm{Na}_{2} \mathrm{O}$ concentration in the stretch case was set at $22 \mathrm{wt} \%$. For the best and conservative cases, a $\mathrm{Na}_{2} \mathrm{O}$ upper limit of 20 wt $\%$ was used.

$\mathrm{B}_{2} \mathrm{O}_{3}$ Of 25 glasses with $\mathrm{B}_{2} \mathrm{O}_{3}$ concentrations less than $3 \mathrm{wt} \%$, only four passed all product quality constraints; two of the four showed gross crystallinity after slow cooling. Therefore, a lower limit of $3 \mathrm{wt} \% \mathrm{~B}_{2} \mathrm{O}_{3}$ was set for all three cases. Since $\mathrm{B}_{2} \mathrm{O}_{3}$ comes primarily from additives (not waste), a nominal $5 \mathrm{wt} \%$ value for $\mathrm{B}_{2} \mathrm{O}_{3}$ was used to formulate the glasses for all three cases to be consistent with the largest fraction of glasses passing all constraints.

$\mathrm{ZrO}_{2}$ Zirconia is a major glass additive, along with $\mathrm{B}_{2} \mathrm{O}_{3}$ and soil. Based on the results of the tree analysis, a lower limit of $6.37 \mathrm{wt} \%$ would be imposed. However, as an additive, a $\mathrm{ZrO}_{2}$ value of $7 \mathrm{wt} \%$ was used for formulation purposes.

$\mathrm{Al}_{2} \mathrm{O}_{3}$ Based on the tree analysis, an upper limit of $10 \mathrm{wt} \% \mathrm{Al}_{2} \mathrm{O}_{3}$ was initially selected for the conservative case. This limit, however, resulted in a highly restricted glass composition region with unrealistically low loading estimates because of the high $\mathrm{Al}_{2} \mathrm{O}_{3}$ in the soil. A review of the glass data in Table 6.2 showed that glasses with $\mathrm{Al}_{2} \mathrm{O}_{3}$ concentrations as high as $16 \mathrm{wt} \%$ passed all constraints if $\mathrm{Na}_{2} \mathrm{O}$ concentrations were $\leq 18 \mathrm{wt} \%$. However, only two glasses with $\mathrm{Na}_{2} \mathrm{O}$ concentrations $>18 \mathrm{wt} \%$ and $\mathrm{Al}_{2} \mathrm{O}_{3}$ concentrations $>10 \mathrm{wt} \%$ passed all constraints. Therefore, the conservative-case upper limit on $\mathrm{Al}_{2} \mathrm{O}_{3}$ was set to 16 wt $\%$ if $\mathrm{Na}_{2} \mathrm{O}$ was $\leq 18 \mathrm{wt} \%$, and $10 \mathrm{wt} \%$ if $\mathrm{Na}_{2} \mathrm{O}$ was $>18 \mathrm{wt} \%$. For the best and stretch cases, the upper limit of $16 \mathrm{wt} \% \mathrm{Al}_{2} \mathrm{O}_{3}$ was used.

$\mathrm{SO}_{3}$ The potential for the accumulation of an alkali-sulfate-based salt accumulation in the ICV melter increases with increased $\mathrm{SO}_{3}$ concentration in the melter feed. The salt accumulation by itself would not cause a problem for the process or product quality. However, the predominantly $\mathrm{Na}_{2} \mathrm{SO}_{4}$ salt potentially will also contain significant quantities of pertechnetate, chromate, and other radioactive or hazardous components. Since the salt is generally water-soluble, these phases will readily dissolve and release $\mathrm{Cr}$, Tc, etc. for transport. Because the potential for the accumulation of a salt phase cannot be readily determined based on crucible glass melts (Darab et al. 2001 and Vienna et al. 2004, for examples), data from scaled ICV tests are required to determine the appropriate concentration limits for $\mathrm{SO}_{3}$ in the ICV melter feed. Engineering-scale ICV tests with $\mathrm{SO}_{3}$ concentrations of $0.83 \mathrm{wt} \%$ (on a glass basis, assuming $100 \%$ retention) were found to successfully immobilize the LAW without the accumulation of a molten salt. Higher concentrations have not been tested. Therefore, the conservative case has a constraint of $\mathrm{SO}_{3} \leq 0.83 \mathrm{wt} \%$. For the best and stretch cases, a higher $\mathrm{SO}_{3}$ concentration is appropriate. This higher concentration was chosen, somewhat arbitrarily, to be $1.5 \mathrm{wt} \%$, which is the solubility of $\mathrm{SO}_{3}$ in commercial soda-aluminosilicate glasses melted at relatively high temperature 
Table 6.2. Summary of ICV Test Glass Compositions, in $\mathrm{Wt} \%^{(\mathrm{a}, \mathrm{b})}$

\begin{tabular}{|c|c|c|c|c|c|c|c|c|c|c|c|c|c|c|c|}
\hline tudy & & & $\mathbf{A l}_{2} \mathbf{O}_{3}$ & $\mathbf{B}_{2} \mathbf{O}_{3}$ & & & $\mathbf{F}$ & & 20 & $\mathbf{P}_{2} \mathbf{O}_{5}$ & $\overline{\mathrm{O}_{2}}$ & $\mathrm{TiO}_{2}$ & $\mathrm{~J}_{2} \mathrm{Cl}$ & $\mathbf{r}_{2} \mathbf{O}_{3} \mathbf{F}$ & $\overline{\mathbf{O}_{3}}$ \\
\hline itial & & $\mathrm{v}$ & 12.04 & 0.00 & 4.62 & 7.80 & 2.14 & 1.20 & 17.00 & 0.57 & 52.48 & & \begin{tabular}{l|l|l|}
0.00 & 0.14 \\
\end{tabular} & \begin{tabular}{ll|l}
0.07 & 0.1
\end{tabular} & 0.67 \\
\hline ial & & V & & 0 & & & & & & & & & 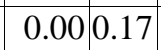 & & 0.8 \\
\hline ial & & $\mathrm{v}$ & & 0 & 4 & 6 & & & & & & & 00 & & 0.95 \\
\hline itial & & v & .78 & 0.00 & 4.05 & 6.84 & 1.92 & 05 & 6.00 & 75 & 5.00 & 05 & 000.24 & 24 & 1.10 \\
\hline itial & & $\mathrm{V}$ & .90 & 0.00 & 4.15 & 7.00 & 1.94 & 08 & 0.00 & 0.61 & 47.07 & 1.08 & \begin{tabular}{|l|l|l|}
5.00 & 0.18 \\
\end{tabular} & 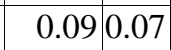 & 0.82 \\
\hline tial & & $\mathrm{V}$ & 90 & 50 & 15 & .00 & 84 & 8 & 0.00 & 61 & .07 & 1 & 018 & & 0.8 \\
\hline itial & & $\mathrm{v}$ & 48 & 00 & 96 & 6.68 & 1.86 & 03 & 0 & 67 & 44.92 & 1.03 & \begin{tabular}{|l|l|}
5.00 & 0.21 \\
\end{tabular} & & 0.96 \\
\hline itial & & $\mathrm{v}$ & 0.48 & 2.50 & 3.96 & 6.68 & 1.86 & 03 & 3.00 & 0.67 & 44.92 & 1.03 & .500 .21 & 10 & 0.9 \\
\hline itial & & $\mathrm{V}$ & 48 & 0.00 & 4.09 & 6.90 & 1.92 & 1.06 & 3.00 & 0.68 & 46.44 & 1.06 & \begin{tabular}{|l|l|}
5.00 & 0.21 \\
\end{tabular} & .08 & 0.9 \\
\hline itial & & $\mathrm{v}$ & .48 & .00 & 83 & 6.45 & 1.80 & 99 & 3.00 & 67 & 3.41 & 0.99 & \begin{tabular}{|l|l|}
.00 & 0.21 \\
\end{tabular} & & 0.9 \\
\hline ial & & $\mathrm{v}$ & 80 & 00 & 08 & 4.68 & 1.92 & 06 & 0 & 68 & 46.35 & 06 & 0.21 & & .9 \\
\hline itial & & $\mathrm{V}$ & 16 & .00 & 3.83 & 8.68 & 81 & 00 & 00 & 67 & 43.47 & 1.00 & \begin{tabular}{l|l|}
00 & 0.21 \\
\end{tabular} & 0.08 & 0.9 \\
\hline tial & & & 89 & 5.00 & 3.75 & 6.33 & 1.76 & 97 & 0.00 & .60 & 42.55 & 0.97 & \begin{tabular}{|l|l|l|}
7.00 & 0.18 \\
\end{tabular} & .07 & 0.8 \\
\hline itial & & & 46 & 00 & .58 & 6.04 & 68 & 93 & 0 . & J & .61 & 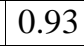 & 0.18 & & 0.8 \\
\hline ial & & $\mathrm{v}$ & 46 & 0 & 3.58 & 04 & 1.68 & 93 & 0 & 59 & .61 & 93 & .18 & $0 /$ & .8 \\
\hline itial & & & 38 & 00 & 3.77 & 6.36 & 76 & 98 & 00 & 53 & 45.75 & 0.98 & 0.15 & 06 & 0.6 \\
\hline ase $\mathrm{i}$ & & & .13 & 5.00 & 2.77 & 4.34 & 1.51 & 35 & 00 & 0.62 & 46.79 & 0.79 & 0.03 & .01 & 0.3 \\
\hline se i & & & .7 & & 06 & 32 & 0 & 77 & 2 & .28 & 65 & 1 & & & 0.8 \\
\hline se i & & & & & 10 & 49 & 0.99 & 77 & & 22 & .79 & 01 & 01 & & 0.1 \\
\hline lase i & & & 40 & 17 & 2.86 & 4.47 & 1.55 & 39 & 00 & 0.63 & 48.19 & 0.82 & 0.03 & 01 & 0.3 \\
\hline ase $\mathrm{i}$ & & $\mathrm{V}$ & .67 & 3.33 & 2.94 & 4.59 & 1.60 & 43 & 00 & .63 & 49.61 & 0.84 & 0.03 & 01 & 0.3 \\
\hline se i & & 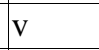 & & & .02 & 2 & 4 & 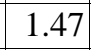 & & & 3 & 10 & & & 0.3 \\
\hline se i & & $\mathrm{v}$ & .20 & & 3.11 & 35 & 69 & & & 64 & 41 & 39 & 03 & & 0.3 \\
\hline ase i & & & 8.88 & 0 & 2.68 & 4.20 & 1.46 & 30 & 00 & 0.67 & 45.29 & 0.77 & 0.03 & 0.01 & 0.41 \\
\hline ase i & & r & 22 & 00 & 2.60 & 4.07 & 1.42 & 26 & 00 & .72 & 3.77 & 0.74 & 04 & 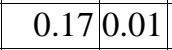 & 0.4 \\
\hline ase i & & & & & 2.74 & 4.21 & 1. & & & 61 & .38 & 0.77 & 03 & & 0.3 \\
\hline se i & & & & 0 & 70 & 08 & 1.42 & 51 & 30 & 59 & .98 & 75 & .03 & & .3 \\
\hline se i & & & & & 2.67 & 95 & 37 & 59 & & 57 & 42.60 & 0.72 & 03 & & 0.3 \\
\hline mulant & & & & 0 & 3.75 & 6.33 & 1.76 & 7 & 0 & ( & 55 & 0.97 & 0.18 & $0.0 \%$ & 0.8 \\
\hline ulant & & & & & 3.77 & & 76 & & & & 75 & 0 & 15 & & 0.6 \\
\hline Iulant & & & .31 & & .94 & 5 & 1.83 & 02 & 0 & 54 & .71 & 02 & 0.15 & & .6 \\
\hline nulant & & & & 00 & 3.62 & 11 & 1.71 & 94 & 00 & 0.64 & 41.11 & 0.94 & 20 & $0 \varepsilon$ & .9 \\
\hline mulant & & $\mathrm{V}$ & & 00 & 3.50 & 5.90 & 1.66 & 91 & 00 & 0.68 & 39.66 & 0.91 & 0.22 & 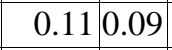 & 1.0 \\
\hline ase ii & & & .00 & 2.00 & 2.50 & 00 & 2.50 & 00 & 00 & .60 & 39.00 & 2.00 & 0.10 & 0.1 & 0.5 \\
\hline ase ii & & & & 00 & 50 & 00 & 2.50 & 00 & 0 & 20 & 44.00 & 2.00 & 0.10 & $n$ & 0.1 \\
\hline phase ii & & S & & 00 & 2.50 & .00 & 2.50 & 0.90 & 00 & 1.60 & 43.85 & 2.00 & 0.10 & 10 & 0.95 \\
\hline hase ii & & $t$, & & 00 & 5.50 & 11.00 & 2.50 & 0.90 & 10 & 0.20 & 39.00 & 2.00 & 0.10 & 0.10 & 0.1 \\
\hline ase ii & & & 50 & & 2.50 & 00 & 2.50 & 3.00 & 23.50 & 0.20 & 39.00 & 2.00 & \begin{tabular}{|l|l|l|}
8.00 & 0.10 \\
\end{tabular} & 0.1 & 0.1 \\
\hline hase ii & & & & 00 & 5.50 & 11.00 & 0.90 & 3.00 & 00 & 0.20 & 46.05 & 0.70 & 2.000 .10 & 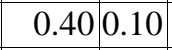 & \\
\hline phase ii & & & 00 & 2.00 & 2.50 & 4.00 & 0.90 & 3.00 & 00 & 0.20 & 54.75 & 2.00 & \begin{tabular}{|l|l|}
3.00 & 0.10 \\
\end{tabular} & 0.10 & 0.95 \\
\hline phase ii & & c & 00 & 2.00 & 5.50 & 4.00 & 0.90 & 0.90 & 9.25 & 1.60 & 47.05 & 2.00 & \begin{tabular}{|l|l|}
8.00 & 0.10 \\
\end{tabular} & 00.10 & 0.10 \\
\hline hase ii & & C & & & 2.50 & 11.00 & 0.90 & 3.00 & 00 & 1.60 & 39.00 & 1.07 & \begin{tabular}{|l|l|}
8.00 & 0.10 \\
\end{tabular} & 0.10 & 0.9 \\
\hline phase ii & & C & 68 & .00 & 5.50 & 4.00 & 2.50 & 3.00 & 18.00 & 1.60 & 39.00 & 0.70 & \begin{tabular}{|l|l|}
4.22 & 0.10 \\
\end{tabular} & 0.1. & .10 \\
\hline phase ii & & C & .95 & 6.00 & 2.50 & 11.00 & 0.90 & 0.90 & 8.00 & 0.20 & 39.00 & 2.00 & \begin{tabular}{ll|l|}
2.75 & 0.10 \\
\end{tabular} & 00.10 & 0.10 \\
\hline hase ii & A & & .00 & 2.00 & 5.50 & 4.00 & 0.90 & 0.90 & 21.49 & 0.20 & 39.00 & 2.00 & \begin{tabular}{|l|l|}
6.36 & 0.10 \\
\end{tabular} & \begin{tabular}{l|l|l}
0.40 & 0.10 \\
\end{tabular} & 0.95 \\
\hline ase ii & 13 & & 05 & 2.00 & 5.50 & 11.00 & 2.50 & 0.90 & 18.00 & 1.60 & 39.00 & 0.70 & $3.95 \mid 0.10$ & $\begin{array}{l}0.400 \\
\end{array}$ & 0.1( \\
\hline
\end{tabular}


Table 6.2. (contd)

\begin{tabular}{|c|c|c|c|c|c|c|c|c|c|c|c|c|c|c|c|}
\hline undy & Glass ID & Type & $\mathbf{A l}_{2} \mathbf{O}_{3}$ & $\overline{\mathbf{B}_{2} \mathbf{O}_{3}}$ & $\mathrm{CaO}$ & $\overline{\mathrm{Fe}_{2} \mathrm{C}}$ & $\overline{{ }_{2} \mathrm{O}}$ & $\overline{M g C}$ & $\overline{\mathrm{a}_{2} \mathrm{O}}$ & $\overline{\mathbf{P}_{2} \mathbf{O}_{5}}$ & $\overline{\mathrm{SiO}_{2}}$ & $\mathrm{TiO}_{2}$ & \begin{tabular}{l|l|l}
$\mathrm{ZrO}_{2}$ & $\mathrm{Cl}$ \\
\end{tabular} & $\overline{\mathrm{Cr}_{2} \mathrm{O}_{3} \mathbf{F}}$ & $\mathbf{S O}_{3}$ \\
\hline hase ii & AN & D,C & 8.07 & 2.00 & .50 & 11.00 & 0.90 & 1.43 & 24.00 & 1. & 39.00 & 0.70 & .00 & \begin{tabular}{|l|l|}
0.40 & 0.10
\end{tabular} & J \\
\hline hase ii & $\mathrm{AN}$ & , C & 8.00 & 2.00 & 5.50 & 4.00 & 2.50 & 3.00 & 23.28 & \begin{tabular}{|l|l|}
30.20 \\
\end{tabular} & 41.17 & 0.70 & .10 & 0.10 & 0.95 \\
\hline hase ii & AMP2-16 & & 8.00 & 6.00 & 5.50 & 4.00 & 0.90 & 3.00 & 20.52 & \begin{tabular}{l|l}
2 & 1.60
\end{tabular} & 44.83 & \begin{tabular}{|l|}
3.00 \\
\end{tabular} & \begin{tabular}{|l|l|l|}
2.85 & 0.10 \\
\end{tabular} & \begin{tabular}{|l|l|l|}
0.40 & 0.10
\end{tabular} & 0.10 \\
\hline
\end{tabular}

(a) The glass type gives a comparison of the property values with the constraints. The notations $\mathrm{t}, \mathrm{p}$, and $\mathrm{v}$ represent glasses that failed the TCLP, PCT, and VHT constraints, respectively. Glasses that showed excessive ( $\geq 10 \mathrm{wt} \%)$ crystallization upon slow cooling were marked with "c".

(b) This table is based upon data that are scheduled to be published in calendar year 2005.

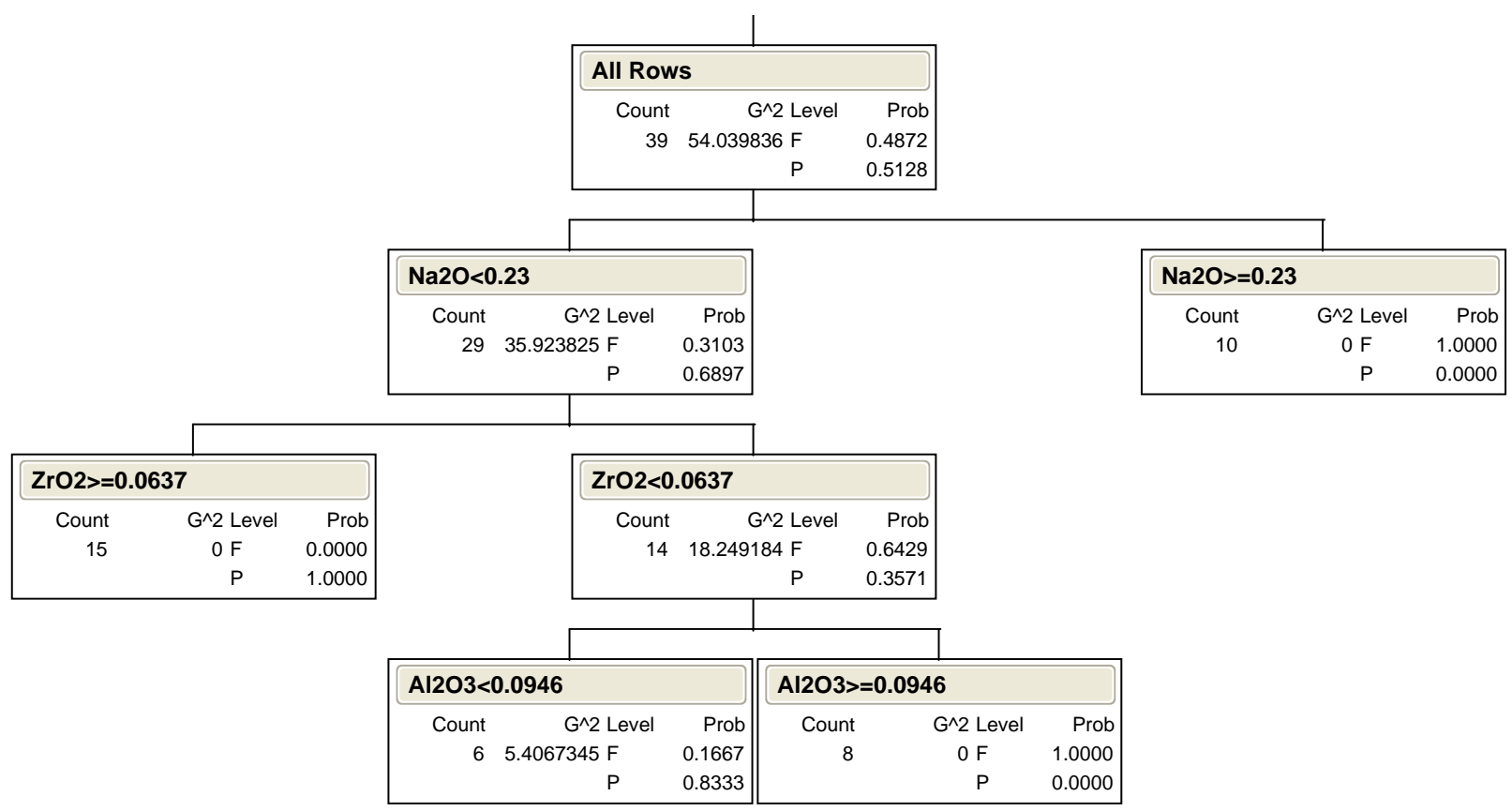

Figure 6.1. Summary of Tree Analyses of the 39 Glasses from Table 6.2 with $\mathrm{SiO}_{2} \geq 40 \mathrm{wt} \%$

(Li et al. 2001). The accumulation of a salt phase in the ICV melter is strongly dependent on the operating parameters in addition to the feed chemistry. It is recommended that the $\mathrm{SO}_{3}$ limit be determined experimentally with scaled ICV tests with a range of likely operating parameters.

$\mathrm{P}_{2} \mathrm{O}_{5} \quad$ Phosphate can influence the ICV process/product quality in four general ways: 1) the interaction between phosphate and sulfate can promote the accumulation of a salt phase in the ICV melter, 2) phosphate can alter the bulk glass properties such as VHT and PCT responses, 3) phosphate can promote immiscible phase separation in the ICV melt, and 4) phosphate can promote crystallization of the melt on slow cooling. As mentioned above, salt accumulation data are relatively scarce for the ICV process. Therefore, a limit on $\mathrm{P}_{2} \mathrm{O}_{5}$ concentration to avoid salt accumulation cannot be derived from ICV test data. Li et al. (1996) showed that crucible melts with $\mathrm{P}_{2} \mathrm{O}_{5}$ concentrations of $>1 \mathrm{wt} \%$, when combined with $\mathrm{SO}_{3}$ concentrations 
of $1 \mathrm{wt} \%$ or greater, showed salt phase separation. Therefore, a conservative-case upper limit on $\mathrm{P}_{2} \mathrm{O}_{5}$ of $1 \mathrm{wt} \%$ was selected. Assuming salt accumulation could be controlled through processing parameters, the best and stretch case $\mathrm{P}_{2} \mathrm{O}_{5}$ limit should be set to avoid immiscible liquid and crystalline phase separation during slow cooling. Jantzen et al. (2000) reviewed phosphate solubility in silicate-based waste glasses. They concluded that glasses with $\mathrm{P}_{2} \mathrm{O}_{5}$ concentrations $>2.5 \mathrm{wt} \%$ were highly prone to either crystalline or immiscible liquid phase separation. Therefore, the best and stretch case upper limits on $\mathrm{P}_{2} \mathrm{O}_{5}$ were set to $2.5 \mathrm{wt} \%$.

$\mathrm{F} \quad$ Fluorine can influence the ICV product properties in the same ways as $\mathrm{P}_{2} \mathrm{O}_{5}$. In addition, $\mathrm{F}$ may significantly impact the useful life of off-gas system components. Fluorine may volatilize during melting, forming an "acid-gas" that can corrode metal off-gas components. The concentrations of F in ICV test glasses, both at crucible and larger scale, ranged up to 0.1 wt\%. This concentration caused no difficulties and would be excessively conservative, even for the conservative case. The conservative case upper concentration limit was set at $0.5 \mathrm{wt} \%$ based on the F concentrations in the Hanford high-level waste (Hrma et al. 1994) and Idaho National Engineering and Environmental Laboratory Sodium Bearing Waste (Scholes et al. 2001) composition variation studies. The best estimate F upper limit of $1 \mathrm{wt} \%$ was based on the preliminary studies for Hanford LAW privatization (Muller and Pegg 1998). The stretch case concentration of $\mathrm{F}$ was arbitrarily set at $1.7 \mathrm{wt} \%$ based on the WTP contract waste loading limits (ORP 2000), which were found to be unrestrictive for nearly all Hanford LAW (as shown in Figure 3.11). These limits should be tested in the ICV process to develop a better basis for the constraints.

Evaluation of the loadings of Hanford low-activity wastes in ICV product with the rules described above resulted in glasses with concentrations of $\mathrm{CaO}, \mathrm{Cl}, \mathrm{Cr}_{2} \mathrm{O}_{3}$, and $\mathrm{K}_{2} \mathrm{O}$ that were sufficiently low that no limits needed to be set on these components. In other words, the best estimated limits for these four components ( $6,0.5,0.5$, and $3 \mathrm{wt} \%$, respectively) would not be exceeded by any glass constrained by the rules described above. The composition constraints that were set for the three cases are summarized in Table 6.3.

\subsection{Glass Mass Estimates}

Using the waste cluster compositions listed in Tables 5.1 through 5.25 and the constraints described in Table 6.3, glass compositions and waste loadings were estimated for each cluster and each case. To formulate the glasses, each waste was combined with the standard additives: $5 \mathrm{wt} \% \mathrm{~B}_{2} \mathrm{O}_{3}, 7 \mathrm{wt} \% \mathrm{ZrO}_{2}$, and sufficient local soil to meet all the composition constraints and requirements. Table 6.4 lists the composition that was used to represent local soil. Although soil composition is known to vary, the variation is relatively small and has been accounted for in the limits set (see Kim et al. 2003 for a description of soil variation and its impact). The concentrations of soil and waste were adjusted so that combined they summed to $88 \mathrm{wt} \%$ of the melter feed (leaving $12 \mathrm{wt} \%$ for $\mathrm{ZrO}_{2}$ [7]and $\mathrm{B}_{2} \mathrm{O}_{3}$ [5]).

The waste loadings resulting from these calculations are summarized in Table 6.5 with the limiting components. To further evaluate the effectiveness of the ICV process, a waste-by-waste comparison was made of the glass volumes that would be produced for each case. The glass masses calculated for each cluster were scaled by the amount of sodium in each that was double-counted (as discussed in Section 3 and tabulated in Table 4.1). The scaled glass masses are listed in Table 6.5 and shown graphically in 
Figure 6.2. These results are summarized in Table 6.6 in terms of the total glass expected to be produced by ICV under each of the three cases.

Table 6.3. Summary of Glass Component Constraints (values in wt $\%$ on a glass basis)

\begin{tabular}{||l|l|l|l||}
\hline Component & \multicolumn{1}{|c|}{ Conservative } & \multicolumn{1}{c|}{ Best } & \multicolumn{1}{c||}{ Stretch } \\
\hline $\mathrm{SiO}_{2}$ & $\geq 40$ & $\geq 40$ & $\geq 39$ \\
\hline $\mathrm{Na}_{2} \mathrm{O}$ & $\begin{array}{l}\leq 20 \text { for } \mathrm{Al}_{2} \mathrm{O}_{3} \leq 10 \\
\leq 18 \text { for } \mathrm{Al}_{2} \mathrm{O}_{3}>10\end{array}$ & $\leq 20$ & $\leq 22$ \\
\hline $\mathrm{B}_{2} \mathrm{O}_{3}$ & $\geq 3$, nominal $=5$ & $\geq 3$, nominal $=5$ & $\geq 3$, nominal $=5$ \\
\hline $\mathrm{ZrO}_{2}$ & $\geq 6.67$, nominal $=7$ & $\geq 6.67$, nominal $=7$ & $\geq 6.67$, nominal $=7$ \\
\hline $\mathrm{Al}_{2} \mathrm{O}_{3}$ & $\leq 16$ for $\mathrm{Na}_{2} \mathrm{O} \leq 18$ & $\leq 16$ & $\leq 16$ \\
& $\leq 10$ for $\mathrm{Na}_{2} \mathrm{O}>18$ & & \\
\hline $\mathrm{SO}_{3}$ & $\leq 0.83$ & $\leq 1.5$ & $\leq 1.5$ \\
\hline $\mathrm{P}_{2} \mathrm{O}_{5}$ & $\leq 1$ & $\leq 2.5$ & $\leq 2.5$ \\
\hline $\mathrm{F}$ & $\leq 0.5$ & $\leq 1$ & $\leq 1.7$ \\
\hline $\mathrm{CaO}$ & $\leq 6$ & $\leq 8$ & $\leq 10$ \\
\hline $\mathrm{K}_{2} \mathrm{O}$ & $\leq 3$ & $\leq 3$ & $\leq 3$ \\
\hline $\mathrm{Cl}$ & $\leq 0.5$ & $\leq 1$ & $\leq 2$ \\
\hline $\mathrm{Cr} \mathrm{O}_{3}$ & $\leq 0.5$ & $\leq 1$ & $\leq 2$ \\
\hline
\end{tabular}

Table 6.4. Soil Composition Used in Glass Calculations (Kim et al. 2003)

\begin{tabular}{||l|c||}
\hline Component & Concentration, wt \% \\
\hline $\mathrm{Al}_{2} \mathrm{O}_{3}$ & 13.96 \\
\hline $\mathrm{B}_{2} \mathrm{O}_{3}$ & 0.00 \\
\hline $\mathrm{CaO}$ & 5.50 \\
\hline $\mathrm{Cl}$ & 0.00 \\
\hline $\mathrm{Cr}_{2} \mathrm{O}_{3}$ & 0.00 \\
\hline $\mathrm{F}$ & 0.00 \\
\hline $\mathrm{Fe}_{2} \mathrm{O}_{3}$ & 9.28 \\
\hline $\mathrm{K}_{2} \mathrm{O}$ & 2.48 \\
\hline $\mathrm{MgO}$ & 1.43 \\
\hline $\mathrm{Na}_{2} \mathrm{O}$ & 3.21 \\
\hline $\mathrm{P}_{2} \mathrm{O}_{5}$ & 0.29 \\
\hline $\mathrm{SiO}_{2}$ & 62.42 \\
\hline $\mathrm{SO}_{3}$ & 0.00 \\
\hline $\mathrm{TiO}_{2}$ & 1.43 \\
\hline $\mathrm{ZrO}_{2}$ & 0.00 \\
\hline $\mathrm{Total}^{2}$ & 100.0 \\
\hline
\end{tabular}


As shown in Table 6.5, much of the glass that was made under standard additive assumptions was limited by the concentrations of $\mathrm{Al}_{2} \mathrm{O}_{3}, \mathrm{SiO}_{2}$, and/or $\mathrm{Na}_{2} \mathrm{O}$, all of which depend on the soil used to make the glass. Therefore, an additional evaluation was performed to determine what the likely loadings and resulting glass masses would be if the additives could be selected specifically to improve waste loading, rather than using only local soil. With the following exceptions, the constraints developed in Section 6.1were used in these calculations:

$\mathrm{Al}_{2} \mathrm{O}_{3}$ Alumina actually helps glass properties at low concentrations, so the acceptable range was changed to $5 \leq \mathrm{Al}_{2} \mathrm{O}_{3} \leq 10 \mathrm{wt} \%$ for the conservative and best cases and $0 \leq \mathrm{Al}_{2} \mathrm{O}_{3} \leq 16 \mathrm{wt} \%$ for the stretch case.

$\mathrm{Na}_{2} \mathrm{O}$ Acceptable soda concentration is a function of the glass composition as a whole. The ability to tailor-fit the additives should allow for higher soda concentrations while maintaining adequate glass properties. The conservative case limit was left at $20 \mathrm{wt} \% \leq \mathrm{Na}_{2} \mathrm{O}$. However, the best estimate and stretch case limits were increased to $21 \mathrm{wt} \% \leq \mathrm{Na}_{2} \mathrm{O}$ and $23 \mathrm{wt} \% \leq$ $\mathrm{Na}_{2} \mathrm{O}$, respectively. 
Table 6.5. Summary of Estimated Waste Loadings and Glass Masses for Standard Additives ${ }^{(\mathrm{a}, \mathrm{b})}$

\begin{tabular}{|c|c|c|c|c|c|c|c|c|c|c|c|}
\hline \multirow[b]{2}{*}{$\begin{array}{c}\text { case } \\
\text { ID }\end{array}$} & \multirow{2}{*}{$\begin{array}{c}\text { Na } \\
\text { Mass } \\
\text { (Mg) }\end{array}$} & \multirow[b]{2}{*}{$\begin{array}{c}\text { Scaling } \\
\text { (\%) }\end{array}$} & \multicolumn{3}{|c|}{ Conservative } & \multicolumn{3}{|c|}{ Best } & \multicolumn{3}{|c|}{ Stretch } \\
\hline & & & $\begin{array}{l}\text { WL } \\
\text { (\%) }\end{array}$ & limits & glass & $\begin{array}{l}\text { WL } \\
\text { (\%) }\end{array}$ & limits & glass & $\begin{array}{l}\text { WL } \\
\text { (\%) }\end{array}$ & limits & glass \\
\hline 1 & 6059 & 100 & 21.4 & $\mathrm{Na}, \mathrm{Al}$ & 51502 & 24.2 & $\mathrm{Na}, \mathrm{Si}$ & 45484 & 25.9 & $\mathrm{Si}$ & 42616 \\
\hline 2 & 1314 & 100 & 12.1 & $\mathrm{P}$ & 20041 & 24.3 & $\mathrm{Si}$ & 9996 & 25.9 & $\mathrm{Si}$ & 9366 \\
\hline 3 & 1715 & 100 & 18.0 & S,P & 18067 & 24.2 & $\mathrm{Si}$ & 13444 & 25.8 & $\mathrm{Si}$ & 12600 \\
\hline 4 & 273 & 100 & 8.6 & $\mathrm{P}$ & 6554 & 24.5 & $\mathrm{Si}$ & 2304 & 26.0 & $\mathrm{P}$ & 2168 \\
\hline 5 & 14 & 100 & 12.4 & $\mathrm{~S}$ & 220 & 22.4 & $S$ & 122 & 22.4 & $\mathrm{~S}$ & 122 \\
\hline 6 & 2169 & 100 & 14.4 & $\mathrm{~S}$ & 27748 & 24.3 & $\mathrm{Si}$ & 16448 & 25.9 & $\mathrm{Si}$ & 15413 \\
\hline 7 & 2571 & 100 & 20.7 & $\mathrm{Na}$ & 21887 & 23.4 & $\mathrm{Na}$ & 19332 & 25.8 & $\mathrm{Si}$ & 17552 \\
\hline 8 & 1467 & 100 & 15.1 & $S$ & 16824 & 23.0 & $\mathrm{Na}$ & 11045 & 25.7 & $\mathrm{Na}$ & 9889 \\
\hline 9 & 184 & 100 & 9.8 & $S$ & 3348 & 17.7 & $S$ & 1854 & 17.7 & $\mathrm{~S}$ & 1854 \\
\hline 10 & 1019 & 100 & 23.3 & $\mathrm{Na}, \mathrm{Al}$ & 8628 & 24.3 & $\mathrm{Si}$ & 8290 & 25.9 & $\mathrm{Si}$ & 7768 \\
\hline 11 & 1089 & 100 & 23.2 & $\mathrm{Na}, \mathrm{Al}$ & 9222 & 24.2 & $\mathrm{Si}$ & 8837 & 25.8 & $\mathrm{Si}$ & 8280 \\
\hline 12 & 1478 & 100 & 13.3 & $\mathrm{P}$ & 19639 & 23.6 & $\mathrm{Na}$ & 11112 & 25.9 & $\mathrm{Si}$ & 10112 \\
\hline 13 & 958 & 100 & 10.9 & $S$ & 15497 & 19.6 & $\mathrm{~S}$ & 8618 & 19.6 & $\mathrm{~S}$ & 8618 \\
\hline 14 & 740 & 100 & 16.5 & $\mathrm{P}$ & 8574 & 24.5 & $\mathrm{Si}$ & 5779 & 26.1 & $\mathrm{Si}$ & 5416 \\
\hline 15 & 2956 & 100 & 19.9 & $\mathrm{Na}$ & 25195 & 22.5 & $\mathrm{Na}$ & 22267 & 25.1 & $\mathrm{Na}$ & 19941 \\
\hline $\mathrm{F}$ & 819 & 100 & 11.6 & $\mathrm{~F}$ & 12290 & 23.2 & $\mathrm{Na}$ & 6158 & 25.9 & $\mathrm{Na}$ & 5515 \\
\hline $\mathrm{O}$ & 4 & 100 & 2.7 & $S$ & 394 & 4.8 & $S$ & 218 & 4.8 & $S$ & 218 \\
\hline $\mathrm{Y} 1$ & 40 & 0 & 1.8 & $\mathrm{P}$ & 0 & 5.4 & $\mathrm{P}$ & 0 & 5.4 & $\mathrm{P}$ & 0 \\
\hline $\mathrm{Y} 2$ & 11 & 100 & 5.9 & $\mathrm{P}$ & 505 & 17.7 & $\mathrm{P}$ & 168 & 17.7 & $\mathrm{P}$ & 168 \\
\hline Y3 & 1186 & 68 & 10.8 & $S$ & 13289 & 19.6 & $\mathrm{~S}$ & 7353 & 19.6 & $\mathrm{~S}$ & 7353 \\
\hline $\mathrm{Y} 4$ & 212 & 0 & 5.2 & $S$ & 0 & 9.4 & $S$ & 0 & 9.4 & $S$ & 0 \\
\hline Y5 & 5691 & 32 & 14.1 & $S$ & 20608 & 21.4 & $\mathrm{Na}$ & 13607 & 23.9 & $\mathrm{Na}$ & 12184 \\
\hline Y6 & 85 & 100 & 3.1 & $S$ & 6587 & 5.5 & $\mathrm{~S}$ & 3645 & 5.5 & $\mathrm{~S}$ & 3645 \\
\hline Y7 & 204 & 0 & 2.8 & $\mathrm{P}$ & 0 & 8.4 & $\mathrm{P}$ & 0 & 8.4 & $\mathrm{P}$ & 0 \\
\hline Y8 & 463 & 100 & 4.4 & $S$ & 23393 & 7.9 & $S$ & 12944 & 7.9 & $\mathrm{~S}$ & 12944 \\
\hline $\mathrm{AB}$ & 27998 & 100 & 21.2 & $S$ & 238106 & 24.0 & $\mathrm{Na}$ & 210292 & 25.8 & $\mathrm{Si}$ & 195149 \\
\hline
\end{tabular}

(a) WL is the waste loading, defined as wt\% of ICV glass that came from waste. The limits include concentrations limits for $\mathrm{Al}_{2} \mathrm{O}_{3}(\mathrm{Al}), \mathrm{F}, \mathrm{Na}_{2} \mathrm{O}(\mathrm{Na}), \mathrm{P}_{2} \mathrm{O}_{5}(\mathrm{P}), \mathrm{SiO}_{2}(\mathrm{Si})$, and $\mathrm{SO}_{3}(\mathrm{~S})$. The mass of sodium in each cluster and the resulting glass mass are in megagrams $(\mathrm{Mg})$.

(b) $\mathrm{AB}$ is a weighted mass average composition of all clusters after scaling for the mass of Na that was doublecounted from the low-curie salt tanks as described in Section 4. It is approximately the same as the composition of the "ST Feed" in Table 4.1. 


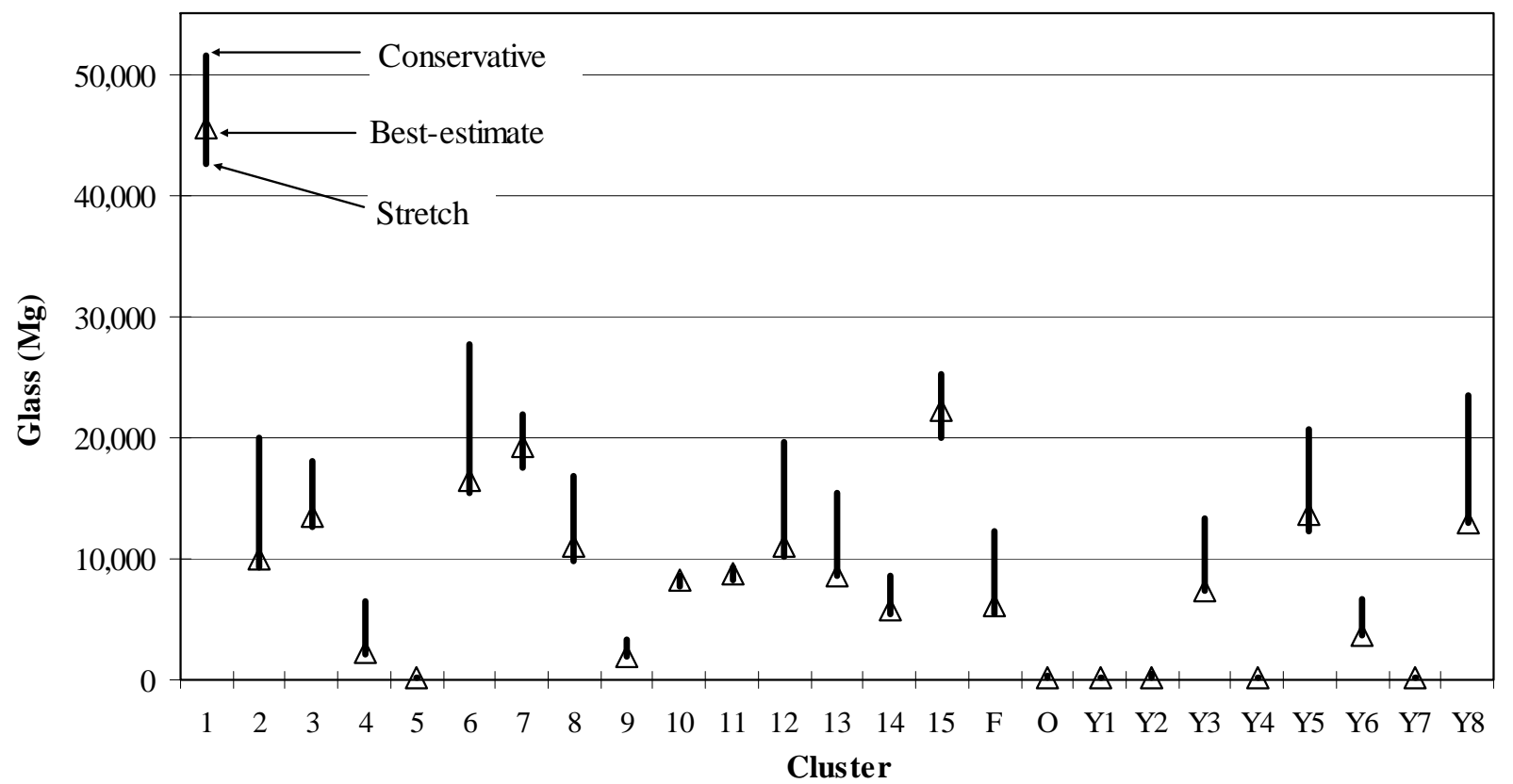

Figure 6.2. $\quad$ Plot of Glass Mass Estimate Ranges for Standard Additives

Table 6.6. $\quad$ Estimated Total Glass Mass for Standard Additives

\begin{tabular}{|l|c|c||}
\hline Case & $\begin{array}{c}\text { Glass Mass Totaled } \\
\text { By Cluster } \\
(\mathrm{Mg})\end{array}$ & $\begin{array}{c}\text { Glass Mass for the } \\
\text { All-blend } \\
(\mathrm{Mg})\end{array}$ \\
\hline Stretch & 213743 & 195149 \\
\hline Best & 229026 & 210292 \\
\hline Conservative & 330012 & 238106 \\
\hline
\end{tabular}

The estimated amounts of glass were calculated by the same method as the standard-additives case described above, except that the additives included soil, pure $\mathrm{SiO}_{2}, \mathrm{~B}_{2} \mathrm{O}_{3}$ (5 wt\%), and $\mathrm{ZrO}_{2}$ (7 wt\%). As before, the sum of all these components was maintained at $100 \%$, and the waste fraction was increased until a limit was met.

The waste loadings, limitations met, and glass masses resulting from these calculations are summarized in Table 6.7. The estimated glass mass ranges are shown in Figure 6.3. The total glass expected to be produced by ICV under each of the three cases is given in Table 6.8. 
Table 6.7. Summary of Estimated Waste Loadings and Glass Masses for Alternative Additives ${ }^{(\mathrm{a}, \mathrm{b})}$

\begin{tabular}{|c|c|c|c|c|c|c|c|c|c|c|c|}
\hline \multirow[b]{2}{*}{\begin{tabular}{|c} 
case \\
ID \\
\end{tabular}} & \multirow{2}{*}{$\begin{array}{c}\text { Na } \\
\text { Mass } \\
\text { (Mg) }\end{array}$} & \multirow[b]{2}{*}{$\begin{array}{c}\text { Scaling } \\
(\%)\end{array}$} & \multicolumn{3}{|c|}{ conservative } & \multicolumn{3}{|c|}{ best } & \multicolumn{3}{|c|}{ stretch } \\
\hline & & & $\begin{array}{l}\text { WL } \\
\text { (\%) }\end{array}$ & limits & glass & $\begin{array}{l}\text { WL } \\
\text { (\%) }\end{array}$ & limits & glass & $\begin{array}{l}\text { WL } \\
(\%)\end{array}$ & limits & glass \\
\hline 1 & 6059 & 100 & 26.8 & $\mathrm{Na}, \mathrm{Al}$ & 41059 & 28.3 & $\mathrm{Na}, \mathrm{Al}$ & 38996 & 31.0 & $\mathrm{Na}$ & 35509 \\
\hline 2 & 1314 & 100 & 14.5 & $\mathrm{P}$ & 16724 & 28.3 & $\mathrm{Na}$ & 8563 & 31.5 & $\mathrm{Na}$ & 7701 \\
\hline 3 & 1715 & 100 & 18.1 & $\mathrm{~S}$ & 17979 & 29.4 & $\mathrm{Na}$ & 11050 & 32.4 & $\mathrm{Na}$ & 10052 \\
\hline 4 & 273 & 100 & 10.6 & $\mathrm{P}$ & 5336 & 28.0 & $\mathrm{P}$ & 2013 & 28.0 & $\mathrm{P}$ & 2013 \\
\hline 5 & 14 & 100 & 12.4 & S & 221 & 22.4 & S & 122 & 22.4 & $\mathrm{~S}$ & 122 \\
\hline 6 & 2169 & 100 & 14.4 & S & 27732 & 26.0 & S & 15345 & 26.0 & $\mathrm{~S}$ & 15345 \\
\hline 7 & 2571 & 100 & 25.5 & $\mathrm{Na}$ & 17711 & 26.9 & $\mathrm{Na}$ & 16821 & 30.0 & $\mathrm{Na}$ & 15068 \\
\hline 8 & 1467 & 100 & 15.1 & $S$ & 16776 & 26.2 & $\mathrm{Na}$ & 9681 & 27.4 & $\mathrm{~S}$ & 9283 \\
\hline 9 & 184 & 100 & 9.8 & S & 3344 & 17.7 & S & 1851 & 17.7 & $\mathrm{~S}$ & 1851 \\
\hline 10 & 1019 & 100 & 29.3 & $\mathrm{Na}$ & 6868 & 30.8 & $\mathrm{Na}$ & 6541 & 33.7 & $\mathrm{Na}$ & 5972 \\
\hline 11 & 1089 & 100 & 23.4 & S & 9141 & 30.6 & $\mathrm{Na}$ & 6990 & 33.5 & $\mathrm{Na}$ & 6383 \\
\hline 12 & 1478 & 100 & 15.8 & $\mathrm{P}$ & 16576 & 26.8 & $\mathrm{Na}$ & 9762 & 30.2 & $\mathrm{Na}$ & 8663 \\
\hline 13 & 958 & 100 & 10.9 & $S$ & 15537 & 19.6 & $S$ & 8597 & 19.6 & $S$ & 8597 \\
\hline 14 & 740 & 100 & 20.2 & $\mathrm{P}$ & 7019 & 29.8 & $\mathrm{Na}$ & 4750 & 32.6 & $\mathrm{Na}$ & 4337 \\
\hline 15 & 2956 & 100 & 24.4 & $\mathrm{Na}$ & 20480 & 25.7 & $\mathrm{Na}$ & 19445 & 28.9 & $\mathrm{Na}$ & 17323 \\
\hline $\mathrm{F}$ & 819 & 100 & 11.7 & $\mathrm{~F}$ & 12170 & 23.4 & $\mathrm{~F}$ & 6085 & 29.7 & $\mathrm{Na}$ & 4797 \\
\hline $\mathrm{O}$ & 4 & 100 & 2.7 & $S$ & 394 & 4.8 & $S$ & 218 & 4.8 & $S$ & 218 \\
\hline Y1 & 40 & 0 & 2.1 & $\mathrm{P}$ & 0 & 5.7 & $\mathrm{P}$ & 0 & 6.0 & $\mathrm{P}$ & 0 \\
\hline $\mathrm{Y} 2$ & 11 & 100 & 7.2 & $\mathrm{P}$ & 414 & 19.3 & $\mathrm{P}$ & 154 & 19.3 & $\mathrm{P}$ & 154 \\
\hline Y3 & 1186 & 68 & 10.8 & $\mathrm{~S}$ & 13289 & 19.6 & S & 7353 & 19.6 & S & 7353 \\
\hline Y4 & 212 & 0 & 5.2 & S & 0 & 9.4 & S & 0 & 9.4 & $S$ & 0 \\
\hline Y5 & 5691 & 32 & 14.1 & $\mathrm{~S}$ & 20608 & 24.0 & $\mathrm{Na}$ & 12158 & 25.5 & $S$ & 11403 \\
\hline Y6 & 85 & 100 & 3.1 & S & 6587 & 5.5 & S & 3645 & 5.5 & $\mathrm{~S}$ & 3645 \\
\hline Y7 & 204 & 0 & 3.3 & $\mathrm{P}$ & 0 & 8.9 & $\mathrm{P}$ & 0 & 9.2 & $\mathrm{P}$ & 0 \\
\hline Y8 & 463 & 100 & 4.4 & S & 23393 & 7.9 & S & 12944 & 7.9 & S & 12944 \\
\hline $\mathrm{AB}$ & 27998 & 100 & 21.1 & $\mathrm{~S}$ & 239192 & 27.6 & $\mathrm{Na}$ & 161351 & 30.7 & $\mathrm{Na}$ & 145517 \\
\hline \multicolumn{12}{|c|}{$\begin{array}{l}\text { (a) WL is the waste loading, defined as wt\% of ICV glass that came from waste. The limits include concentrations } \\
\text { limits for } \mathrm{Al}_{2} \mathrm{O}_{3}(\mathrm{Al}), \mathrm{F}, \mathrm{Na}_{2} \mathrm{O}(\mathrm{Na}), \mathrm{P}_{2} \mathrm{O}_{5}(\mathrm{P}), \mathrm{SiO}_{2}(\mathrm{Si}) \text {, and } \mathrm{SO}_{3}(\mathrm{~S}) \text {. The mass of sodium in each cluster and the } \\
\text { resulting glass mass are in megagrams }(\mathrm{Mg}) \text {. } \\
\text { (b) AB is a weighted mass average composition of all clusters after scaling for the mass of Na that was double- } \\
\text { counted from the low-curie salt tanks as described in Section } 4 \text {. It is approximately the same as the composition of } \\
\text { the "ST Feed" in Table 4.1. }\end{array}$} \\
\hline
\end{tabular}




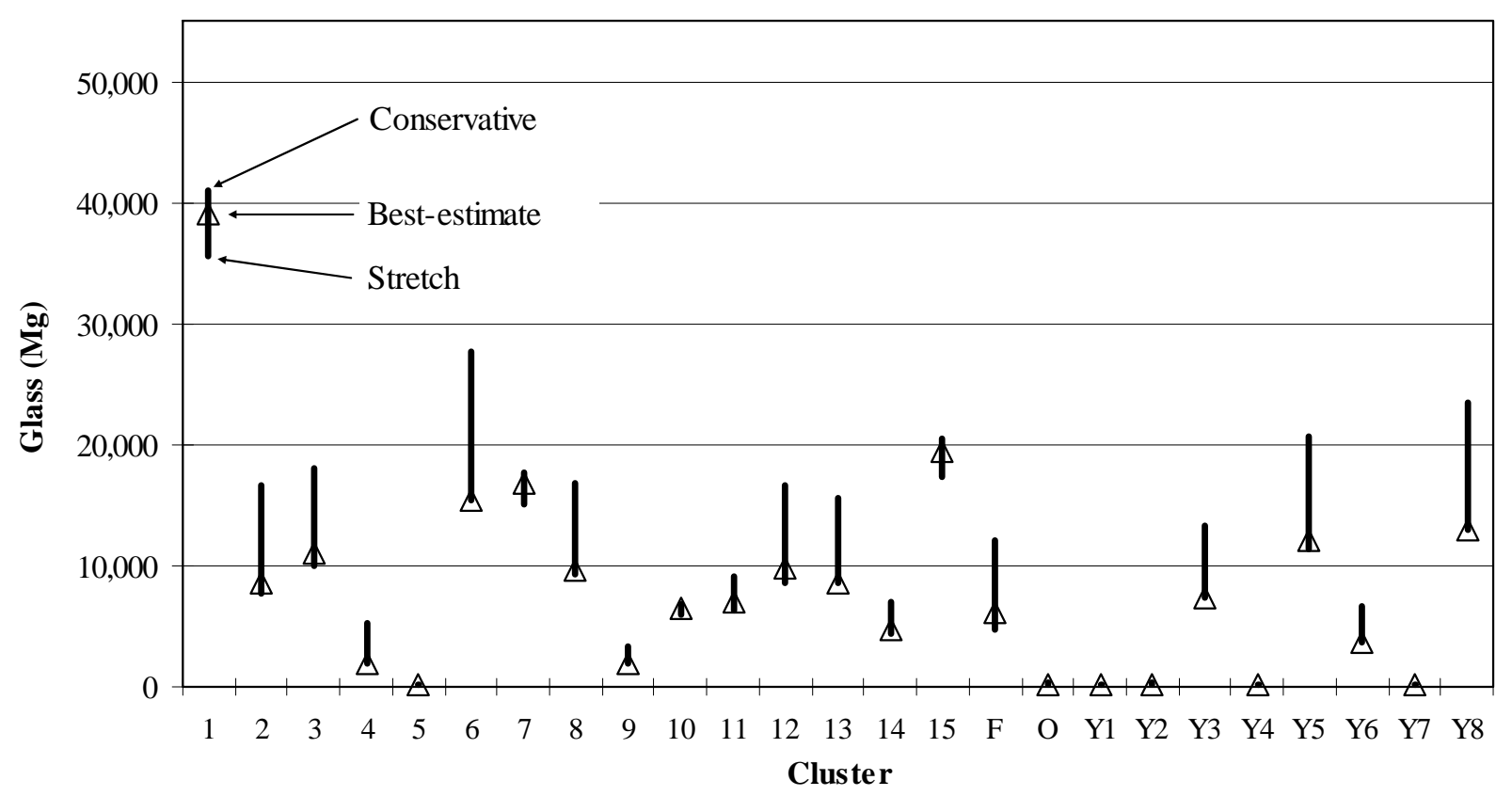

Figure 6.3. Plot of Glass Mass Estimate Ranges for Alternative Additives

Table 6.8. Estimated Total Glass Mass for Alternative Additives

\begin{tabular}{|l|c|c|}
\hline Case & $\begin{array}{c}\text { Glass Mass Totaled } \\
\text { By Cluster } \\
(\mathrm{Mg})\end{array}$ & $\begin{array}{c}\text { Glass Mass for the } \\
\text { All-blend } \\
\text { (Mg) }\end{array}$ \\
\hline Stretch & 188733 & 145517 \\
\hline Best & 203084 & 161351 \\
\hline Conservative & 299358 & 239192 \\
\hline
\end{tabular}

\subsection{Conclusions}

Certain conclusions can be drawn from these glass mass calculations:

- The present state of knowledge about the effects of glass composition on glass performance and processing can lead to uncertainties of roughly $45 \%$ in glass production, based on the range between "conservative" and "stretch" production estimates.

- The lowest waste loadings result from high sulfur and phosphorus concentrations.

- The extremes in the composition of the waste fed to ICV increase the total glass mass by $10 \%$ to $30 \%$, based on a comparison of the total glass calculated by summing the glass from individual 
PNNL-14985 Rev. 0

cluster compositions to the total glass calculated for the "all-blends" composition (averaged over the entire feed).

- Adjusting the glass additives with pure $\mathrm{SiO}_{2}$ to optimize waste loading decreases the glass mass (summed over all individual clusters) by $10 \%$ to $15 \%$. 


\subsection{Conclusions}

The purposes of this letter report were to describe the compositional variability of the feed to ST, show which compositions are most relevant to ICV testing, and make an initial assessment of the performance of ICV in handling the ST feed. The feed envelope was considered to include both the WTP-pretreated waste streams, which contribute most of the Na in the feed, and the low-curie SST wastes that are designated to be pretreated outside the WTP. The feed streams were statistically grouped into "clusters," each containing streams with similar concentrations of selected constituents, and the amount of glass that ICV would produce from each cluster was estimated based on three different sets of waste loading assumptions that ranged from lower-bound to upper-bound waste loading.

As the first step in analysis, the distributions of the predicted concentrations of $\mathrm{Al}_{2} \mathrm{O}_{3}, \mathrm{CaO}, \mathrm{Cl}, \mathrm{F}$, $\mathrm{MoO}_{3}, \mathrm{NO}_{2}+\mathrm{NO}_{3}, \mathrm{P}_{2} \mathrm{O}_{5}$, and $\mathrm{SO}_{3}$ in the population of oxidized waste streams were reviewed. The maximum concentrations of $\mathrm{MoO}_{3}$ and $\mathrm{CaO}$ were found to be quite low, respectively 0.25 and $0.032 \mathrm{wt} \%$ at a nominal $\mathrm{Na}_{2} \mathrm{O}$ concentration of $20 \mathrm{wt} \%$. As a result of this finding, $\mathrm{CaO}$ and $\mathrm{MoO}_{3}$ were deemed not important enough to use in defining clusters.

The concentrations of significant components in glasses tested in the past were compared to the distribution of concentrations in the ST feed. The maximum nominal concentrations of $\mathrm{CaO}$ and $\mathrm{SO}_{3}$ that have been tested in glasses, as of November 2004, are about equal to the Na-weighted averages for all feed streams. (Here "nominal" refers to the concentration calculated on a basis of $20 \mathrm{wt} \% \mathrm{Na}_{2} \mathrm{O}$.) $\mathrm{The}$ maximum tested nominal concentration of $\mathrm{NO}_{2}+\mathrm{NO}_{3}$ is an upper bound for streams containing more than $99 \%$ of the $\mathrm{Na}$ in the feed. However, most of the $\mathrm{Na}$ in the feed is associated with nominal concentrations of $\mathrm{Al}_{2} \mathrm{O}_{3}, \mathrm{Cl}, \mathrm{F}$, and $\mathrm{P}_{2} \mathrm{O}_{5}$ that are higher than any tested concentrations, suggesting that it would be useful to test more extreme compositions.

The WTP-derived streams whose composition was dominated by WTP off-gas product (SBS condensate plus WEPS liquid) had high concentrations of the volatiles $\mathrm{Cl}, \mathrm{F}, \mathrm{NO}_{2}+\mathrm{NO}_{3}$, and $\mathrm{SO}_{3}$, but contained very little of the feed $\mathrm{Na}$ (less than $0.1 \%$ of the total). The predicted waste loading for the cluster containing these streams was quite low, 3 to $5 \mathrm{wt} \%$, owing to its high $\mathrm{SO}_{3}$ content.

It was found that a cluster of streams that were derived predominantly from WTP-pretreated LAW and that had relatively high $\mathrm{F}$ content (above $0.745 \mathrm{wt} \%$, nominal basis) tended also to have lower $\mathrm{Al}_{2} \mathrm{O}_{3}$ and $\mathrm{SO}_{3}$ than other WTP LAW streams. This cluster, containing about $3 \%$ of the total Na, had a predicted waste loading of 12 to $26 \mathrm{wt} \%$; it was the only cluster whose loading was limited primarily by constraints on F concentration.

The remaining waste streams were divided into 15 clusters derived from low-F WTP-pretreated LAW waste and 8 clusters derived from the low-curie SST wastes. The two types of wastes were not mingled in clusters because the nominal $\mathrm{Al}_{2} \mathrm{O}_{3}, \mathrm{SO}_{3}$, and $\mathrm{P}_{2} \mathrm{O}_{5}$ concentrations of the low-curie tank wastes frequently fell outside the ranges of the concentrations in pretreated LAW streams generated by the WTP pretreatment facility. Several low-curie wastes were higher in nominal $\mathrm{P}_{2} \mathrm{O}_{5}$ and $\mathrm{SO}_{3}$ that the WTPderived LAW, and most were lower in $\mathrm{Al}_{2} \mathrm{O}_{3}$ and $\mathrm{Cl}$. In general, the low-curie SST wastes had lower 
allowable waste loadings than the WTP-derived LAW streams; the difference often resulted from constraints on maximum $\mathrm{SO}_{3}$ and $\mathrm{P}_{2} \mathrm{O}_{5}$ loadings.

As noted previously, this study's predictions of glass production were based on a range of assumptions about the constraints of waste loading. The lower bound was based on "conservative" constraints that experience suggests are reasonably likely to be successful. A middle estimate came from "best estimate" constraints. The upper bound was based on "stretch" constraints, the maximum likely loading that could be obtained with sufficient investment in technology development. The difference between the campaign-total glass mass estimated for "stretch" and "conservative" assumptions was about $45 \%$. This can be taken as an estimate of the uncertainty caused by the present state of knowledge about the effect of glass composition on glass performance and processing.

The campaign-total glass production was calculated in two ways: as the sum of the glass produced for each cluster, and as the glass produced for the total feed stream (i.e., using the campaign-average feed composition). The total glass mass calculated in the first way was $10 \%$ to $30 \%$ greater than the second, averaged-composition total. This increase can be taken as the result of the extremes in the composition of the ST feed.

The effect of a change in the glass additives was also considered in the study. The standard additives were $5 \mathrm{wt} \% \mathrm{~B}_{2} \mathrm{O}_{3}, 7 \mathrm{wt} \% \mathrm{ZrO}_{2}$, and sufficient local soil to meet all the composition constraints and requirements. In the non-standard case, the assumed additives were $5 \mathrm{wt} \% \mathrm{~B}_{2} \mathrm{O}_{3}$, 7 wt $\% \mathrm{ZrO}_{2}$, enough pure $\mathrm{SiO}_{2}$ to keep Si from being the limiting constraint, and sufficient local soil to meet all the composition constraints and requirements. The adjustment of the additive with $\mathrm{SiO}_{2}$ decreased the campaign-total glass mass (summed over individual feed clusters) by 10 to $15 \%$. 
PNNL-14985 Rev. 0

\subsection{References}

61 FR 2338. January 25, 1996. U.S. Environmental Protection Agency, "Land Disposal Restrictions Supplemental Proposal to Phase IV: Clarification of Bevill Exclusion of Mining Wastes, Changes to the Definition of Solid Waste for Mineral Processing Waste, Treatment Standards for Characteristic Mineral Processing Wastes, and Associated Issues,” Federal Register.

62 FR 26041. May 12, 1997. U.S. Environmental Protection Agency, "Land Disposal Restrictions Phase IV: Second Supplemental Proposal on Treatment Standards for Metal Wastes and Mineral Processing Wastes, Mineral Processing and Bevill Exclusion Issues, and the Use of Hazardous Wastes as Fill Supplemental Proposed Rule,” Federal Register.

AMEC. 2003. Bulk Vitrification Project \# 17902: Large-Scale Bulk Vitrification Test Results. December 2003. AMEC Earth and Environmental, Inc., Richland, Washington.

American Society for Testing and Materials (ASTM). 2002. "Standard Test Methods for Determining Chemical Durability of Nuclear, Hazardous, and Mixed Waste Glasses and Multiphase Glass Ceramics: The Product Consistency Test (PCT),” C 1285-02, ASTM International, West Conshohocken, Pennsylvania.

Buelt, J. L., C. L. Timmerman, K. H. Oma, V. F. FitzPatrick, and J. G. Carter. 1987. In Situ Vitrification of Transuranic Waste: An Updated Systems Evaluation and Applications Assessment, PNL-4800, Suppl. 1, Pacific Northwest Laboratory, Richland, Washington.

Darab, J. G., D. D. Graham, B. D. MacIsaac, R. L. Russell, D. K. Peeler, H. D. Smith, and J. D. Vienna. 2001. Sulfur Partitioning During Vitrification of INEEL Sodium Bearing Waste: Status Report, PNNL13588, Pacific Northwest National Laboratory, Richland, Washington.

U.S. Environmental Protection Agency (EPA). 1997. Test Methods for Evaluation of Solid Waste Physical/Chemical Methods, SW-846, 3rd. Ed., as amended, U. S. Environmental Protection Agency, Washington, D. C.

Hrma, P., G. F. Piepel, M. J. Schweiger, D. E. Smith, D.-S. Kim, P. E. Redgate, J. D. Vienna, C. A. LoPresti, D. B. Simpson, D. K. Peeler, and M. H. Langowski. 1994. Property/Composition Relationships for Hanford High-Level Waste Glasses Melting at $1150^{\circ} \mathrm{C}$, PNL-10359, Vol. 1 and 2, Pacific Northwest Laboratory, Richland, Washington.

Jantzen, C. M., K. G. Brown, J. B. Pickett, and G. L. Ritzhaupt. 2000. Crystalline Phase Separation in Phosphate Containing Waste Glasses: Relevancy to Vitrification of Idaho National Engineering and Environmental Laboratory (I NEEL) High Activity Waste (U), WSRC-TR-2000-00339, Westinghouse Savannah River Company, Aiken, South Carolina.

Kim, D.-S., J. D. Vienna, P. R. Hrma, M. J. Schweiger, J. Matyáš, J. V. Crum, D. E. Smith, G. J. Sevigny, W. C. Buchmiller, J. S. Tixier, Jr., J. D. Yeager, K. B. Belew. 2003. Development and Testing of ICV Glasses for Hanford LAW, PNNL-14351, Pacific Northwest National Laboratory, Richland, Washington. 
Kirkbride, R.A., G.K. Allen, P.G. Haigh, T.M. Hohl, A.M. Johnson, S.L. Orcutt, and R.S. Wittman. 2003. Tank Farm Contractor Operation and Utilization Plan. HNF-SD-WM-SP-012 Rev. 5, CH2M HILL Hanford Group, Inc., Richland, Washington.

Li, H., P. Hrma, and J. D. Vienna. 2001. "Sulfate Retention and Segregation in Simulated Radioactive Waste Borosilicate Glass,” in Ceramic Transactions, 119, pp. 237-246, American Ceramic Society, Westerville, Ohio.

Li, H., J. G. Darab, D. W. Matson, P. A. Smith, P. Hrma, Y. Chen, and J. Liu. 1996. "Phosphate-Sulfate Interactions in Simulated Low-Level Radioactive Waste Glasses,” in Mat. Res. Soc. Symp. Proc. Vol. 412, Materials Research Society, Pittsburgh, Pennsylvania.

Mann, F. M., K. C. Burgard, W. R. Root, R. J. Puigh, S. H. Finfrock, R. Khaleel, S. H. Bacon, E. J. Freeman, B. P. McGrail, S. K. Wurstner, and P. E. Lamont. 2001. Hanford Immobilized Low-Activity Waste Performance Assessment: 2001 Version, DOE/ORP-2000-24, Rev. 0, U. S. Department of Energy, Office of River Protection, Richland, Washington.

Muller, I. S., and I. L. Pegg. 1998. Glass Formulation and Testing with TWRS LAW Simulants, Final Report for GTS Durateck Inc. and BNFL Inc., Catholic University of America, Washington D.C.

SAS. 2004a. Version 5 User’s Guide for JMP Version 5.1.1. SAS, Cary, North Carolina.

SAS. 2004b. Version 5 Statistics and Graphics Guide for JMP Version 5.1.1. SAS, Cary, North Carolina.

Scholes, B. A., J. D. Vienna, D. K. Peeler, and T. B. Edwards. 2002. The Preparation and Characterization of INTEC Sodium Bearing Waste Phase 1 Composition Variation Study Glasses, INEEL/EXT-02-003686, Idaho National Engineering and Environmental Laboratory, Idaho Falls, Idaho.

Vienna, J. D., P. Hrma, A. Jiricka, D. E. Smith, T. H. Lorier, I. A. Reamer, and R. L. Schulz. 2001. Hanford Immobilized LAW Product Acceptance Testing: Tanks Focus Area Results, PNNL-13744, Pacific Northwest National Laboratory, Richland, Washington.

Vienna, J. D., P. Hrma, W. C. Buchmiller, and J. S. Ricklefs. 2004. Preliminary Investigation of Sulfur Loading in Hanford LAW Glass, PNNL-14649, Pacific Northwest National Laboratory, Richland, Washington. 


\section{Appendix}

\section{Calculation of Low-Curie Waste Streams}


PNNL-14985 Rev. 0

Page 74 of 78 


\section{Appendix}

\section{Calculation of Low-Curie Waste Streams}

Some of the Supplemental Treatment (ST) feed will come from single-shell tanks (SSTs) that have been determined to contain low-curie wastes; these feeds will be pretreated by solid-liquid separation, but not by the Waste Treatment Plant (WTP). These low-curie SST wastes fall into two categories as described below.

First, there are the SST wastes that at one time were considered low-curie wastes and were sent to ST without WTP pretreatment. They were therefore excluded from the TFCOUP5A feed vector that was used in this study to model WTP operations. The excluded tanks were 241-B-101, B-102, B-103, B-105, B-106, B-107, B-108, B-109, BX-103, BX-107, BX-108, BX-109, BX-110, BY-102, BY-105, BY-108, BY-111, BY-112, S-109, and TY-102. This set of 20 tanks must be considered in order to complete the TFCOUP5A feed vector.

In addition, since the time the TFCOUP5A vector was generated, 16 tanks that were not part of the excluded set have been determined to contain low-curie waste. These tanks were BX-111, BY-103, BY109, S-105, T-109, TX-103, TX-105, TX-108, TX-110, TX-111, TX-112, TX-114, TX-115, TX-117, TX118, and U-107. Although the waste in these 16 tanks made up part of the WTP-derived ST feed, this report also considers these new tanks as potentially part of the non-WTP-treated ST feed.

Therefore, this study considers a total of 36 tank wastes as potential low-curie waste feeds. This appendix describes how the low-curie waste streams' compositions were calculated.

\section{A.1 Background}

Saltcake wastes are expected to be retrieved by dissolution, with the dissolution water applied primarily at the top of the waste. This is the same method that has been employed to retrieve waste in tank S-112. In addition, some pretreatment in the form of solid-liquid separation is planned for the lowcurie tank waste retrievals. Thus, it is reasonable to assume that only the soluble portion of the low-curie waste will be sent to ST.

Selective dissolution also has an effect on the ST feed from low-curie tanks. This effect is the result of two mechanisms, the selective removal of species in the original interstitial liquid and the selective removal of the species in the most soluble solids.

The first selective dissolution mechanism was observed in tank S-112; the waste retrieved from this tank contained less and less ${ }^{137} \mathrm{Cs}$ as retrieval progressed. ${ }^{\text {(a) }}$ Because ${ }^{137} \mathrm{Cs}$ was present in the pre-retrieval waste almost exclusively as a dissolved species in the interstitial liquid, the diminishing retrieval of ${ }^{137} \mathrm{Cs}$ implies that the original interstitial liquid was somewhat preferentially removed in the early stages of retrieval. This type of selective removal has been attributed to the tendency for the liquid newly produced

(a) Mahoney, L.A. and G.B. Josephson. April 1, 2004. Analysis of Retrieval Data from Tank 241-S-112. Letter report ST04.15, Pacific Northwest National Laboratory, Richland, Washington. 
near the top of the waste, owing to dissolution, to flush out the original interstitial liquid located in the bottom part of the S-112 waste.

The second mechanism comes into play because the waste retrieved early in the process has a tendency to contain a higher fraction of the more-soluble sodium salts, such as sodium nitrate. Dissolution of the less soluble salts is delayed to later in retrieval. This mechanism results from the common-ion effect: all the soluble salts contain sodium so the more soluble salts force the less soluble out of solution through the mass-action law of solubility products. This effect has been seen in tank waste dissolution studies such as the one conducted by Callaway (2003) with a sample of tank S-102 waste.

During retrieval, the two mechanisms just described cause continuous changes in the composition of the retrieved waste liquid. Most of the ${ }^{137} \mathrm{Cs}$ is expected to leave dissolution-retrieved tanks early; only the waste streams later in the process have low enough activity to be sent directly to ST. This lateretrieval waste can be expected to be relatively low in species that, like ${ }^{137} \mathrm{Cs}$, were present exclusively in the interstitial liquid and relatively high in the anions from less-soluble sodium salts. For the purpose of this study, "selective dissolution" factors are used to represent the composition variation as occurring in only two steps, early and late retrieval. The late-retrieval, low-activity streams are considered to be sent to ST; the early-retrieval, higher-activity streams are not.

\section{A.2 Method}

The current Best Basis Inventory (BBI) and the BBI water wash factors ${ }^{(a)}$ for the wastes in the 36 selected tanks were used as inputs to find the water-soluble inventories in each tank. It was assumed that the water-soluble inventory of each constituent was equal to the product of the water wash factor for the constituent in the tank and the inventory of the constituent in the tank. This simplified approach applies the wash factor to both the liquid and solid phases in the waste, which is not strictly consistent with the definition of the water wash factor as the fraction of a constituent removed, by water, from the bulk solid phase of the tank waste to the liquid phase.

The inconsistency means that water-soluble inventory of a constituent will be underestimated to the extent that 1) its wash factor is less than 1.0, and 2) its tank inventory is found in the liquid. There is no effect for those constituents that have a wash factor equal to or near 1.0, a category that includes $\mathrm{Cl}, \mathrm{F}$, $\mathrm{Na}, \mathrm{NO}_{2}, \mathrm{NO}_{3}, \mathrm{PO}_{4}$, and $\mathrm{SO}_{4}$. These species will end up in the water-soluble portion of the inventory whether they began in the waste liquid or the bulk solids. The inconsistency does lead to a low estimate of constituents that are only partially washable and more present in the liquid inventory than in the bulk solids in the tank. Of these constituents, the most significant in in-container vitrification (ICV) is Al. Its underestimation is not expected to be large compared to the uncertainty in its tank inventory.

BBI water wash factors were available for all the radionuclides in the Tank Characterization Report (TCR) BBI, for all the non-radioactive TCR analytes, and for the supplemental analytes Ag, Cd, Ce, Th, and W. For some tanks, the supplemental BBIs contained analytes for which BBI water wash factors were not available. Such analytes included As, B, Ba, Be, CN, Co, Cu, free OH, Li, Mg, Mo, Nd, Sb, Se,

(a) Tank Waste Information Network System (TWINS), queried October 21, 2004 (36 tanks listed in text: Best Basis Inventory TCR, Calculation Details, Supplemental Analyte Calculation Details, and Wash and Leach Factors), http://twins.pnl.gov/twins.htm. 
$\mathrm{Ti}, \mathrm{V}$, and $\mathrm{Zn}$. This study assumes water wash factors equal to 1.0 (complete solubility in water) for analytes for which no factors were given.

The assumption of complete solubility may overestimate the amounts of the analytes sent to ST. However, most of these (including Mo) are trace species that have no effect on ICV. The exception is free $\mathrm{OH}$, which is not of concern to ICV but is more than a trace constituent. This particular species is water soluble so it is not overestimated by assuming its water wash factor is 1.0.

Because selective dissolution will be used to reduce the activity in low-curie wastes sent to ST, the water-soluble inventories of the constituents must be modified to obtain ST feed streams. A recent study $^{(\mathrm{a})}$ provides the current planning assumptions for selective dissolution separation for chemical species and radionuclides. These assumptions are reproduced in Table A.1.

Table A.1. Separation Percentages for Low-Curie Feed to Supplemental Treatment

\begin{tabular}{|c|c|}
\hline Constituent & $\begin{array}{c}\text { Percentage of Tank Waste to } \\
\text { Supplemental Treatment }\end{array}$ \\
\hline $\mathrm{Al}$ & 40 \\
\hline${ }^{241}$ Am (soluble portion) & 100 \\
\hline $\mathrm{Ca}$ & 100 \\
\hline $\mathrm{CrO}_{4}$ & 45 \\
\hline${ }^{137} \mathrm{Cs}$ & 40 \\
\hline${ }^{129} \mathrm{I}$ & 40 \\
\hline $\mathrm{K}$ & 80 \\
\hline $\mathrm{Na}$ & 70 \\
\hline $\mathrm{NO}_{3}$ & 65 \\
\hline $\mathrm{NO}_{2}$ & 40 \\
\hline $\mathrm{OH}$ & 40 \\
\hline $\mathrm{Cl}$ & 40 \\
\hline $\bar{F}$ & 100 \\
\hline $\mathrm{PO}_{4}$ & 75 \\
\hline Pu (soluble portion) & 100 \\
\hline $\mathrm{SO}_{4}$ & 90 \\
\hline${ }^{90} \mathrm{Sr}$ (soluble portion) & 100 \\
\hline${ }^{99} \mathrm{Tc}$ & 40 \\
\hline $\mathrm{TIC}\left(\mathrm{CO}_{3}\right)$ & 85 \\
\hline TOC & 90 \\
\hline
\end{tabular}

Table A.1 shows that the constituents present entirely, or almost entirely, in dissolved form are largely removed before the waste is directed to ST. These include $\mathrm{Al}, \mathrm{CrO}_{4},{ }^{137} \mathrm{Cs},{ }^{129} \mathrm{I}, \mathrm{NO}_{2}, \mathrm{OH}$, and ${ }^{99}$ Tc. Only $40-45 \%$ of these constituents is left behind for ST feed. Constituents that are much less

(a) Raymond, R.E. October 20, 2004. Candidate Single-Shell Tanks for Low-Curie Feed to Supplemental Treatment, letter report 7F300-04-RER-001 R1, CH2MHILL Hanford Group, Inc. 
soluble are assumed to dissolve only later in the retrieval process, after the retrieved waste has been directed to ST. As a consequence, $80-100 \%$ of these constituents is sent to ST.

The referenced study ${ }^{(a)}$ did not state any selective dissolution assumptions for many of the analytes in the TCR and supplemental BBIs. Therefore, this study used general solubility criteria that were consistent with those reflected in Table A.1 to assign selective dissolution fractions to other constituents. These assignments, and the bases for them, are shown in Table A.2.

The inventory of each constituent that was ST feed was calculated as the product of the water-soluble inventory and the selective dissolution factor. These ST feed inventories gave the compositions used to carry out cluster analysis and glass calculations for the 36 low-curie ST feed streams.

Table A.2. Supplemental Separation Percentages for Low-Curie Feed to ST

\begin{tabular}{|c|c|c|}
\hline Constituent & $\begin{array}{c}\text { Percentage of Tank Waste } \\
\text { to Supplemental } \\
\text { Treatment }\end{array}$ & Reason Percentage Was Chosen \\
\hline $\begin{array}{l}\text { Ag, Be, Bi, Cd, Ce, Co, Cu, } \\
\text { Fe, Hg, La, Mg, Mn, Nd, Ni, } \\
\text { Pb, Sb, Si, Th, Ti, U, V, W, } \\
\text { Zn, Zr } \\
\text { (soluble portions) }\end{array}$ & 100 & $\begin{array}{l}\text { The small soluble fractions of these nearly } \\
\text { insoluble constituents are assumed to result } \\
\text { from dissolution late in retrieval and } \\
\text { therefore are sent entirely to ST. }\end{array}$ \\
\hline B, Ba, CN, Li, Mo, Se & 40 & $\begin{array}{l}\text { These trace constituents are more soluble } \\
\text { and are assumed to be entirely dissolved, } \\
\text { and therefore are mostly removed with the } \\
\text { original interstitial liquid, as is }{ }^{137} \mathrm{Cs} \text {. } \\
\text { Therefore the same factor is used as for } \\
{ }^{137} \text { Cs. }\end{array}$ \\
\hline Free $\mathrm{OH}$ & 40 & $\begin{array}{l}\text { Constituent is present entirely in interstitial } \\
\text { liquid. }\end{array}$ \\
\hline $\begin{array}{l}\text { Almost all radionuclides } \\
\text { (exceptions are given below) }\end{array}$ & 100 & $\begin{array}{l}\text { Treated as nearly insoluble because of their } \\
\text { small water wash factors. }\end{array}$ \\
\hline${ }^{3} \mathrm{H}$ & 40 & Present only in interstitial liquid (water). \\
\hline${ }^{14} \mathrm{C}$ & 85 & Same as TIC. \\
\hline${ }^{134} \mathrm{Cs},{ }^{137 \mathrm{~m}} \mathrm{Ba}$ & 40 & Same as ${ }^{137}$ Cs. \\
\hline${ }^{79} \mathrm{Se}$ & 40 & Same as Se. \\
\hline
\end{tabular}

\section{A.3 References}

Callaway, W.S. 2003. Tank 241-S-102 Core Sample Dissolution Testing Report, RPP-15940, Fluor Hanford, Inc., Richland, Washington. 